\title{
Avalanche Dynamics and Magnetoelastic Coupling in Metallic Glasses
}

\author{
Dissertation \\ zur Erlangung des mathematisch-naturwissenschaftlichen \\ Doktorgrades \\ " Doctor rerum naturalium " \\ der Georg-August-Universität Göttingen \\ im Promotionsprogramm ProPhys \\ der Georg-August University School of Science (GAUSS)
}

vorgelegt von

\section{Carlos Herrero Gómez}

aus Madrid

Göttingen, 2017 
Betreuungsausschuss

Prof. Dr. Konrad Samwer

I. Physikalisches Institut, Georg-August-Universität Göttingen

Prof. Dr. Cynthia Volkert

Institut für Materialphysik, Georg-August-Universität Göttingen

Mitglieder der Prüfungskommision

Referent:

Prof. Dr. Konrad Samwer

I. Physikalisches Institut, Georg-August-Universität Göttingen

Korreferentin:

Prof. Dr. Cynthia Volkert

Institut für Materialphysik, Georg-August-Universität Göttingen

Weitere Mitglieder der Prüfungskommision

Prof. Dr. Vasily Moshnyaga

I. Physikalisches Institut, Georg-August-Universität Göttingen

Prof. Dr. Michael Seibt

IV. Physikalisches Institut, Georg-August-Universitäat Göttingen

Dr. Claus Heussinger

Institut für Theoretische Physik, Georg-August-Universitäat Göttingen

Dr. Richard Vink

Institut für Materialphysik, Georg-August-Universität Göttingen

Tag der mündlichen Prüfung: 24 Januar 2018 


\section{Contents}

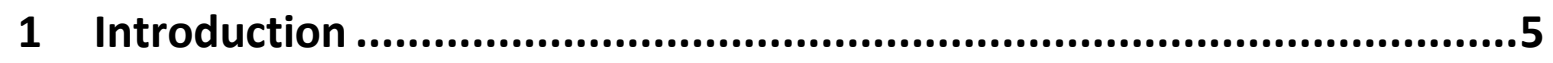

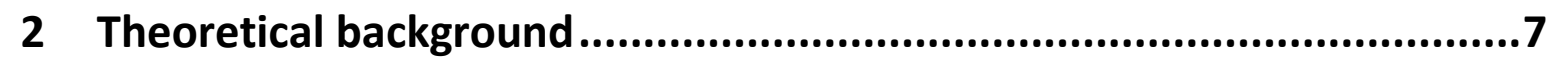

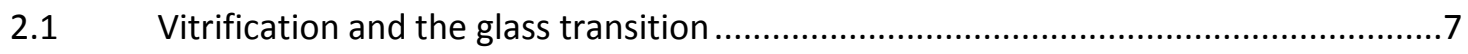

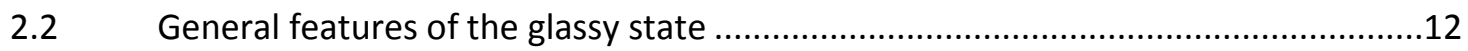

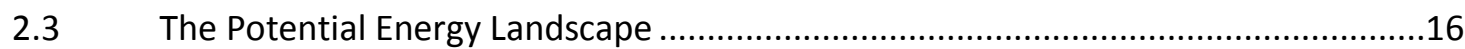

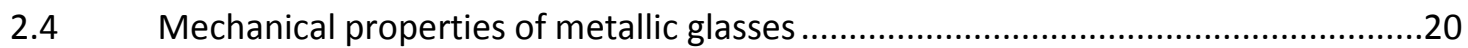

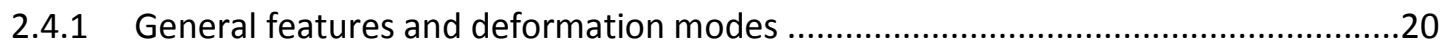

2.4.2 Microscopic origin of plasticity on metallic glasses ...............................................23

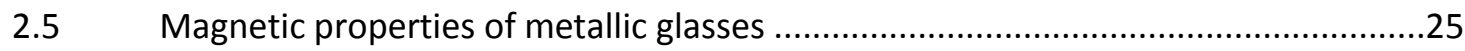

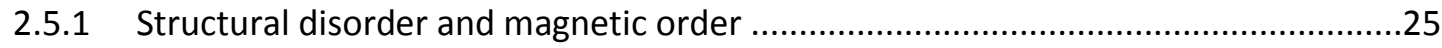

2.5.2 Magnetostriction: Local origin and macroscopic effect............................................26

2.6 Crackling noise, avalanche dynamics and intermittency ......................................29

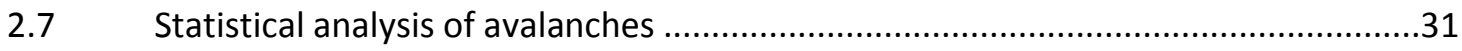

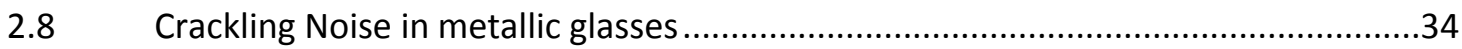

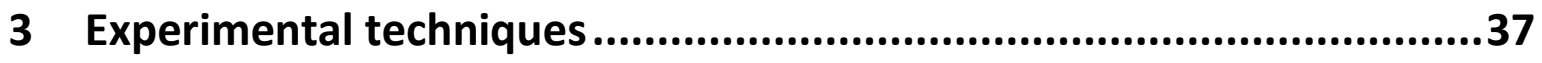

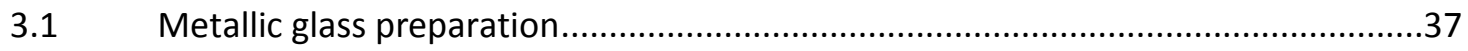

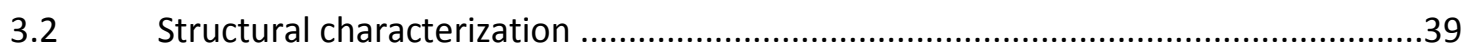

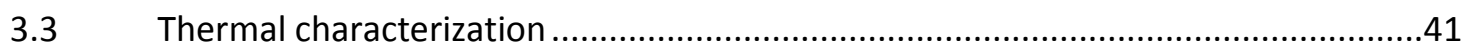

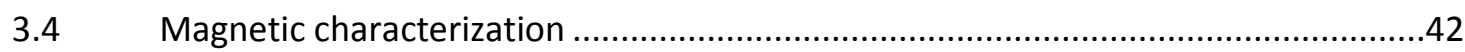

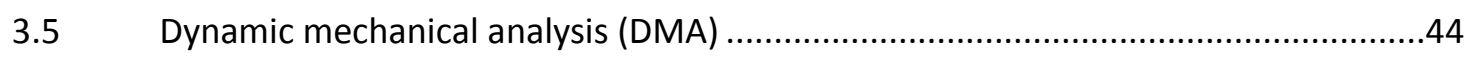

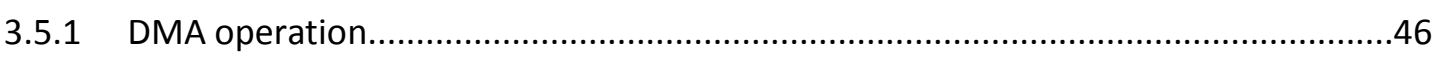

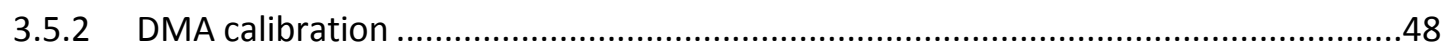

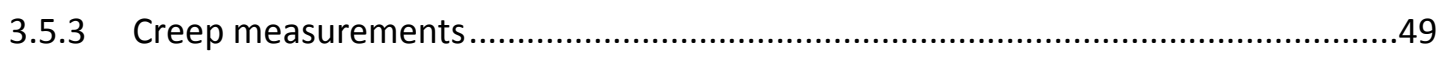

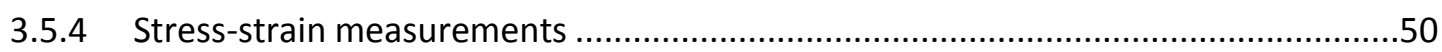




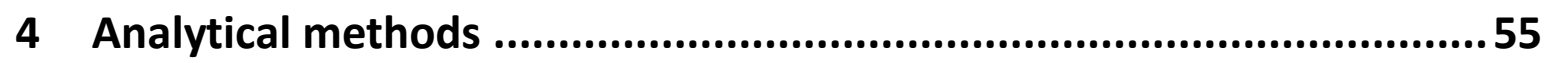

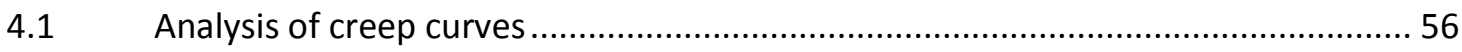

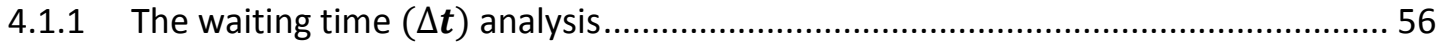

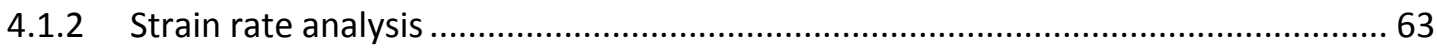

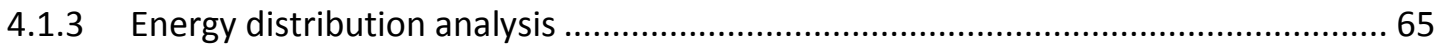

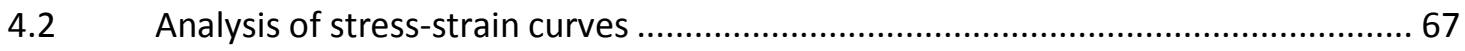

4.2.1 The effective modulus analysis ............................................................... 67

4.2.2 Avalanche analysis in stress-strain curves: the renormalized modulus.................. 69

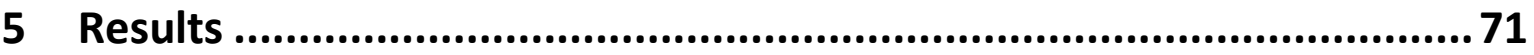

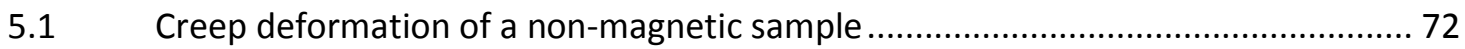

5.2 Creep deformation of a magnetic glass under stress and magnetic driving............ 80

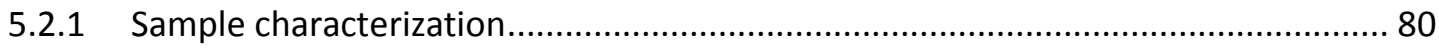

5.2.2 The influence of temperature on creep deformation........................................ 85

5.2.3 The influence of mechanical stress on creep deformation................................ 90

5.2.4 The influence of magnetic field intensity on creep deformation ......................... 94

5.2.5 The influence of magnetic field orientation on creep deformation:

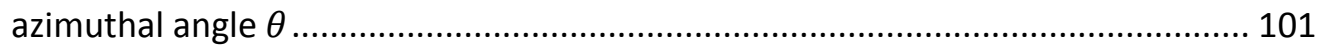

5.2.6 The influence of magnetic field orientation on creep deformation:

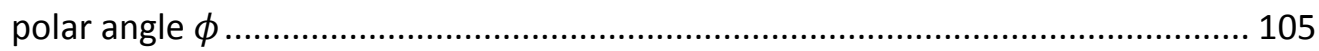

5.3 Stress-strain measurements of magnetic glasses under magnetic driving............ 109

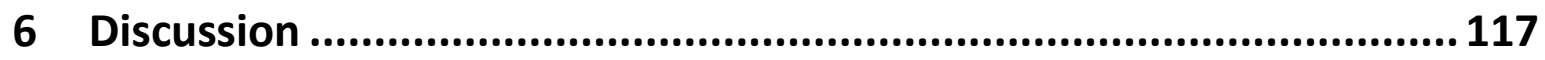

6.1 Creep deformation of a non-magnetic sample ............................................ 117

6.1.1 Characterization of sample properties....................................................... 117

6.1.2 Crossover in the waiting time distribution.............................................. 117

6.1.3 The influence of stress and temperature on the waiting time distribution shape. 120

6.1.4 Physical interpretation of the waiting times.............................................. 121

6.2 Creep deformation in magnetic metallic glasses ......................................... 123

6.2.1 Characterization of sample properties..................................................... 123

6.2.2 The influence of stress and temperature on creep deformation ....................... 124

6.2.3 The influence of the magnetic field on creep deformation ............................. 125

6.3 Magnetoelastic coupling in stress-strain measurements .............................. 129 


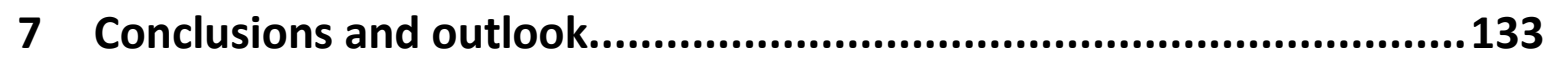

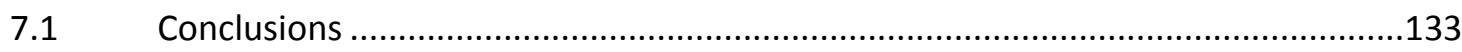

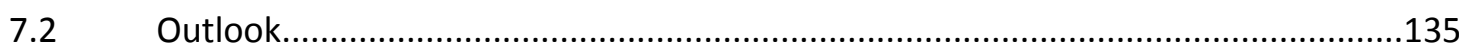

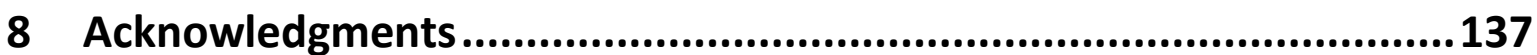

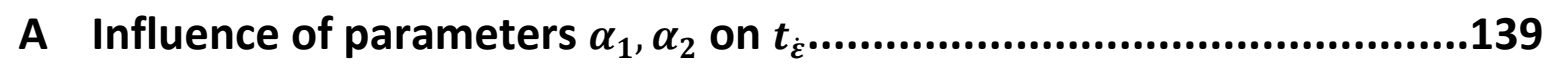

B Evaluation of $W$ as fragility estimator......................................................141

C Magnetic domains in ribbons under tensile loading.............................142

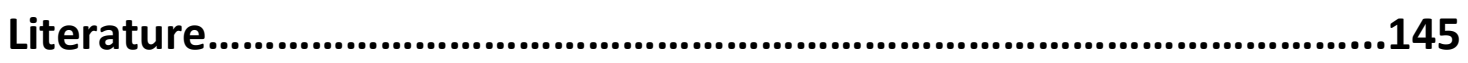

List of figures........................................................................................................155

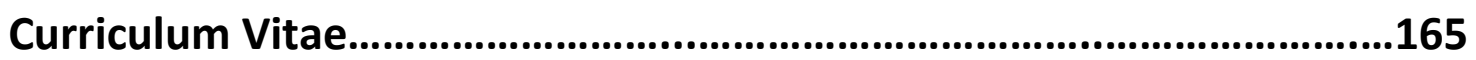

Publications and conferences..................................................................166 


\section{Introduction}

Amorphous materials were already known by the early civilizations on Earth. In fact, it is believed that the first contact of mankind with glasses was the use of obsidian by primitive men both in weapons and decorative goods. Later on, the first glass-preparation was reported by Phoenician sailors, who accidentally melted soda blocks during lunchtime, giving rise to the first glass preparation around 5000 B.C ${ }^{1}$. However, it was not until 600 B.C. when the earliest written testimonies of glassmaking procedures were reported in the clay tables of King Assurbanipal of Mesopotamia ${ }^{2}$. Apart from some questionable advices regarding sheep sacrifices and gods worshipping, much of the instructions given back in that table are still valid and currently applied.

Glasses can be formed by different experimental techniques, such as sputtering deposition ${ }^{3}$, laser deposition ${ }^{4}$ or melt quenching ${ }^{5}$. Regardless of the differences among these techniques, the common goal of all of them is to avoid long range ordering during the formation of the solid. Recently, in 1960, Klement et al., managed to quench a metallic liquid into the glassy state, forming the first metallic glass ${ }^{6}$. By means of that technique, an alloy melt is cooled down with cooling rate high enough to avoid crystallization. Ever since, preparation techniques have rapidly developed and a wide range of metallic glasses are nowadays available in bulk ${ }^{7,8}$ which have attracted much interest both from the fundamental point of view and from the perspective of practical applications. Mechanically, metallic glasses were found to exhibit higher elastic limit ${ }^{9}$, wear corrosion ${ }^{10}$ and lower damping ${ }^{11}$ than many other materials including their crystalline counterparts. Such properties have motivated their implementation in sporting goods, mechanical actuators springs and luxury items ${ }^{12}$. On the other hand, metallic glasses made of magnetic elements were found to show exceptionally soft magnetic behavior ${ }^{13,14}$. That fact, together with their high electrical resistivity, motivated their massive implementation in magnetic cores of electrical transformers ${ }^{15,16}$. Additionally, magnetic metallic glasses show outstanding magnetomechanical coupling, which motivated their use as sensors and actuators ${ }^{17,18,19}$.

Regardless of their well-known macroscopic mechanical and magnetic behavior, the microscopic origin of macroscopic phenomena remains only partially understood to this day. In this context, Argon postulated the local deformation unit in amorphous matter as the so-called Shear Transformation Zone (STZ) ${ }^{20}$. Argon's concept of Shear Transformation Zones was merged to the Potential Energy Landscape framework of Goldstein ${ }^{21}$ by Samwer and Johnson ${ }^{22}$. According to that model, a glass accommodates stress by the collective shear of tens of atoms. Such cluster forms the plastic core of the STZ, whose shearing induces an Eshelby stress field with quadrupolar symmetry ${ }^{23}$. Such quadrupolar field is able to soften other spots and therefore induce a cascade deformation event or avalanche ${ }^{24}$. The ability of a triggered STZ to substantially modify the 
surrounding elastic matrix, generating avalanches of STZ's, or on other words the cooperativity among STZ' $\mathrm{s}^{25}$ is a key parameter that governs the macroscopic deformation behavior of metallic glasses, which ranges from ductile to brittle depending on the experimental conditions ${ }^{26}$. However, the way the dynamics among STZ's take place remains unclear, and the topology and cooperativity of their interactions are still debated ${ }^{27}$.

In this context, the deformation of metallic glasses has been extensively studied from the point of view of avalanche dynamics during the last two decades. Many experimental $\left.\right|^{28,29,30}$ and theoretica| ${ }^{31,24,32}$ evidence hint at deformation events exhibiting typical features of avalanche processes, as is the wide distribution of scales and intermittency ${ }^{33,34}$. There is thus hope that statistical analysis of the intermittent deformation signal and careful comparison against different models, would shed light into features which still remain elusive, as for instance the topology and cooperativity of the deformation process.

This work is devoted to the study of metallic glasses by means of a statistical analysis of the intermittent deformation signal. The first part of the memoir deals with the analysis of avalanches produced during creep deformation of non-magnetic $\mathrm{Pd}_{77.5} \mathrm{Cu}_{6} \mathrm{Si}_{16.5}$ ribbons. A crossover in the avalanche statistics observed by Krisponeit et $\mathrm{al}^{30}$ is analyzed under a wide range of experimental conditions. The underlying transition is physically interpreted in terms of the macroscopic strain rate and inertia ${ }^{35}$. The second part of the work focuses on the analysis of the avalanches produced during creep deformation of magnetostrictive Fe-rich 2605SA1 glasses from Metglas Inc. A crossover was also observed in these samples, which suggest the universality of that transition. Furthermore, avalanches under different magnetic field intensities and orientations were also analyzed. Analysis of experiments under magnetic fields suggest an interplay between magnetic domain walls and STZ's which affects the crossover transition and the dynamic heterogeneities in the glass. Specifically, a certain magnetic domain wall orientation along $45^{\circ}$ with respect to the applied stress seems to maximize the regime of inertial avalanches and dynamic heterogeneities. Finally, the last part of this memoir deals with the analysis of the magnetomechanical coupling during stress-strain measurements at room temperature. Those measurements were performed on two alloys: highly magnetostrictive $2605 \mathrm{SA} 1$ and $\mathrm{Fe}_{2} \mathrm{Co}_{73} \mathrm{Si}_{10} \mathrm{~B}_{15}$ with vanishing magnetostriction in it unstrained state. The results obtained were interpreted both from a macroscopic approach (by means of the analysis of the macroscopic Young modulus as a function of the magnetic field) and from the point of view of the avalanches (by an analysis of the intermittent deformation signal). The main magnetomechanical effect appears to be governed by magnetic wall rotation upon stress increase, which leads to a non-linear addition of the magnetic and mechanical energies.

This work is divided into seven chapters. After an introduction, Chapter 2 provides a summary of general features of glasses, including the glass transition and an overall description of their mechanical and magnetic properties. In particular, the phenomenon of the magnetomechanical coupling and its physical origin is treated as well. An introduction is also given to the field of avalanche dynamics, and the state of the art of avalanche analysis in metallic glasses is overviewed there. In Chapter 3 the different experimental equipment used to produce the data analyzed throughout this memoir are presented, with particular emphasis on the Dynamic Mechanical Analyzer and a modification thereof that was implemented to include magnetic field driving. The analytical methods employed for the analysis of such data in the context of avalanche dynamics are then described in Chapter 4. Chapter 5 presents the experimental results, which are subsequently discussed in detail in Chapter 6 . To conclude, in Chapter 7 the main conclusions of this work are summarized, and a few suggestions for possible future research are gathered. 


\section{Theoretical background}

\subsection{Vitrification and the glass transition}

Amorphous materials in general, and glasses in particular, are characterized by the lack of long range order, in sharp contrast with crystals whose main feature is the presence of an ordered microscopic lattice.

When a glass forming liquid is cooled below its freezing point $\mathrm{T}_{\mathrm{m}}$ it may crystallize through a first order transition into its crystalline phase. This is due to the fact that at $\mathrm{T}_{\mathrm{m}}$ the condition for thermodynamic equilibrium is achieved by the crystalline phase. Such condition is given by the minimization of its free energy

$$
\mathrm{F}=\mathrm{U}-\mathrm{TS}
$$

where $\mathrm{U}$ and $\mathrm{S}$ are respectively the internal energy and entropy and $\mathrm{T}$ the absolute temperature. In principle, one might expect a minimum of $\mathrm{F}$ to correspond to a minimum of $\mathrm{U}$ and a maximum of $\mathrm{S}$, but the derivatives of $\mathrm{U}$ and $\mathrm{S}$ with respect to the temperature have generally the same sign. Therefore, the temperature plays the key role of determining the weight of each of the components of the free energy. At very high temperatures the entropy term governs the free energy and the system is at equilibrium in its liquid phase, which is characterized by high entropy and internal energy. The freezing point is defined as the temperature at which the internal energy term becomes dominant for the minimization of the free energy. If that is the case, the system undergoes crystallization in equilibrium, since this is the configuration that minimizes the internal energy, at the expense of minimizing the entropy as well. It should be recalled that crystallization is a first order transition since many physical quantities as the enthalpy or volume show a discontinuity at that point.

The aforementioned considerations only apply in the case of thermodynamic equilibrium. If the melt is cooled fast enough, the high viscosity increase rate hampers crystallization and the system is driven into the so-called super cooled liquid regime ${ }^{36,37}$. As the system is quenched further, it eventually falls out of equilibrium and forms a glass (metastable disordered solid), through the socalled glass transition ${ }^{38,39}$. 
Figure 2.1 depicts the vitrification process by showing schematically the temperature dependence on volume and enthalpy. The temperature range at which volume and enthalpy derivatives with respect to the temperature change, defines the glass transition temperature $\mathrm{T}_{\mathrm{g}}$. The glass transition temperature $\mathrm{T}_{\mathrm{g}}$ is also defined as the temperature at which characteristic relaxation times reach $100 \mathrm{~s}^{38,40}$.

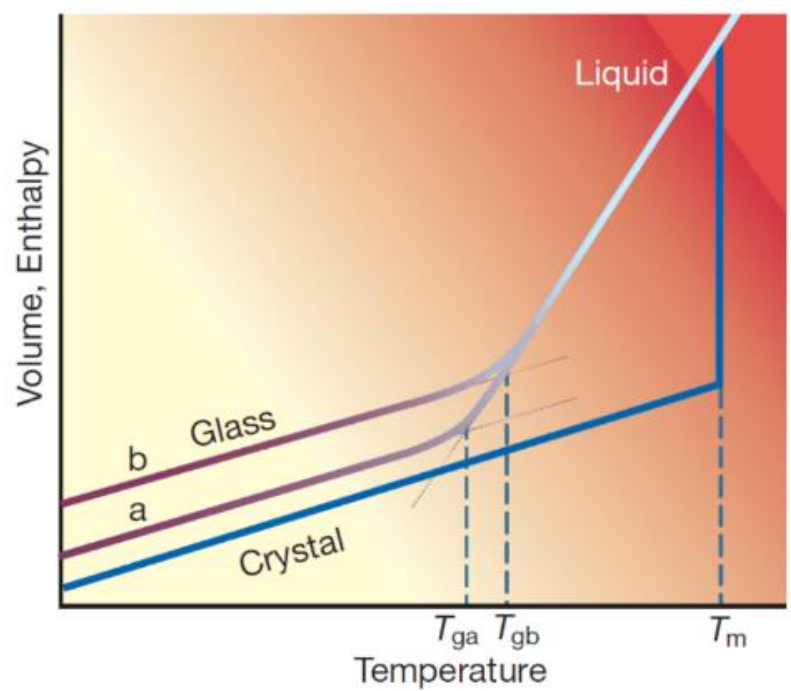

Figure 2.1 Temperature dependence of a liquid volume or entalphy at constant pressure. $^{38}$

The glass transition temperature depends on the cooling rate and so does the final glassy state. $\mathrm{T}_{\mathrm{g}}$ increases with the cooling rate, since as the latter increases it becomes more challenging for the system to relax at a comparable rate ${ }^{38,41,42}$. In Figure 2.1, $\mathrm{T}_{\mathrm{ga}}$ and $\mathrm{T}_{\mathrm{gb}}$ represent two temperatures of the glass transition accomplished with cooling rates $\dot{\mathrm{T}}_{\mathrm{a}}$, $\dot{\mathrm{T}}_{\mathrm{b}}$ respectively, where $\left|\dot{\mathrm{T}}_{\mathrm{a}}\right|<\left|\dot{\mathrm{T}}_{\mathrm{b}}\right|$. The energy branch of the glass depends on the temperature at which the undercooled liquid falls out of equilibrium, i.e., its glass temperature. An undercooled liquid quenched at higher rates would fall out of equilibrium at higher temperatures and at higher energy levels than the same liquid quenched at lower rates. In Figure 2.1 the glassy branch denoted as (b) represents a preparation path with higher cooling rate than branch (a).

The glass transition is also related to the ergodicity of the system, i.e., the ability of the material to explore the whole configurational space. In that sense, the glass transition can be understood as a crossover from an ergodic state in the supercooled liquid regime, in which the system is able to explore the majority of the configurational space (except the configuration which correspond to the crystal), to a glassy non ergodic state in which the system is trapped in a given configurational state $^{38}$. That means that the configurational degrees of freedom are sharply reduced at the glass transition. That fact is reflected in a reduction of the specific heat $\mathrm{C}_{\mathrm{p}}^{\text {glass }}<\mathrm{C}_{\mathrm{p}}^{\text {liq }}$.Specific heat at the supercooled liquid phase is dominated by configurational degrees of freedom, whereas the main contribution of the specific heat at the glassy state are only vibrational degrees of freedom ${ }^{43}$.

Since a glass is in a metastable state, its configuration is continuously evolving towards lower energetic configurations ${ }^{44}$. Such a process is called aging or structural relaxation, and it is of outmost importance since aging also involves the evolution of mechanical, magnetic or chemical properties of the glass ${ }^{45,26}$. Typically aging is negligible at temperatures $\mathrm{T} \ll \mathrm{T}_{\mathrm{g}}$, but it starts to play a role as the temperature approaches $\mathrm{T}_{\mathrm{g}}{ }^{44}$. On the other hand, a glass can be externally driven in order to bring 
it back to its original state. Such a process is called rejuvenation, and can be done by mechanical treatments ${ }^{46}$, or cyclic cryogenic baths ${ }^{47,48}$. A special kind of glasses are the so called ultra-stable glasses. Such glasses are prepared at a low enough cooling rate so the system achieves a low deep minima in the configurational space ${ }^{3,49}$. Recent findings seem to indicate that in the case of ultrastable glasses a heat treatment below Tg would not induce aging, but rather a process known as anti-aging, characterized by an increase of the potential energy, due to the temperature treatment ${ }^{50}$.

It has been already mentioned that the viscosity $\eta$ increases dramatically at the vicinity of the glass transition, which results in an increase of the relaxation times and in the eventual formation of the glass. The functional dependence of the viscosity with the temperature upon cooling is used to classify the different glass formers ${ }^{51}$. More precisely, the glass formers are termed as "strong" if the change of viscosity follows an Arrhenius law, of the form:

$$
\eta(T)=\eta_{0} \exp \left(\frac{E}{R T}\right)
$$

where $\mathrm{R}$ is the universal gas constant and $\mathrm{E}$ represents a constant activation Energy. A canonical example of strong glass former are $\mathrm{SiO}_{2}$ glasses (common window glass). On the other hand, the glasses are termed as "fragile" if their viscosity follows the Vogel-Fulcher-Tammann law (VFT), given by the expression:

$$
\eta(T)=\eta_{0} \exp \left(\frac{\mathrm{DT}_{0}}{\left(\mathrm{~T}-\mathrm{T}_{0}\right)}\right)
$$

Where $\mathrm{D}, \eta_{0}$ and $\mathrm{T}_{0}$ are constants. O-terphenyl and toluene are two classical examples of fragile glasses.

Angell proposed a way to represent the viscosity data which allows to easily classify the glass formers between strong and fragile ${ }^{51}$. The so-called Angell-plot consists on the representation of the logarithm of the viscosity as a function of $\frac{\mathrm{T}_{\mathrm{g}}}{\mathrm{T}}$. An example of such representation can be found in Figure 2.2 in which the strong liquids can be identified as those that follow the upper straight line, whereas fragile ones depart from such linear behavior. 


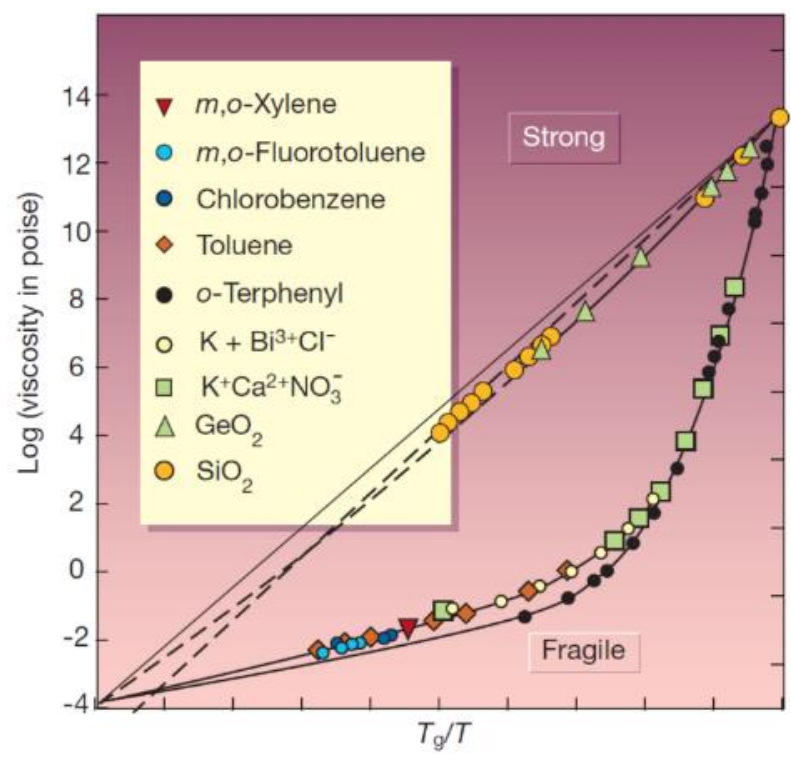

Figure 2.2 Angell representation of the logarithm of glass former viscosities as a function of $\frac{T_{g}}{T}$. Strong glass formers show linear behavior in such scaling, whereas fragile glasses deviate from linearity ${ }^{38}$.

The fragility of a glass former can be quantified by means of the fragility index $\mathrm{m}^{52}$, given by the expression:

$$
m=\frac{d(\log (\eta))}{d\left(\frac{T}{T_{g}}\right)}
$$

evaluated at $\mathrm{T}=\mathrm{T}_{\mathrm{g}}$

The fragility is proportional to the increase of viscosity when approaching the glass transition. Fragile systems present a sharp increase of the viscosity in the vicinity of $\mathrm{T}_{\mathrm{g}}$, while for strong glass formers the viscosity increase is constant and governed by an Arrhenius behavior.

More recently, an analytic relationship between fragility and viscosity of metallic glasses and their interatomic potential has been established ${ }^{53}$. According to such model, which reproduces successfully experimental data, the viscosity can be expressed in a double exponential closed-form function of only one free parameter, the atomic volume $V_{c}$, by means of the following expression:

$$
\frac{\eta(T)}{\eta_{0}}=\exp \left\{\frac{V_{c} C_{G}}{K T} \exp \left[(2+\lambda) \alpha_{T} T_{g}\left(1-\frac{T}{T_{g}}\right)\right]\right\}
$$

where $C_{G}$ is a prefactor independent of T, $\alpha_{T}$ the expansion coefficient and $\eta_{0}$ a normalization constant. The parameter $\lambda$ represents the steepness of the inter-atomic potential and can be 
estimated from fitting the leftmost flank of the first peak of radial distribution function, by means of a power-law approximation $g(r) \sim(r-\sigma)^{\lambda}{ }^{54}$. Such steepness of the leftmost flank of $g(r)$ determines the fragility of the system, since metallic glasses with steeper repulsive part of the interatomic interaction are found to be more fragile than those with softer repulsions. The softness or steepness of the interatomic potential is mainly determined by the electronic configuration of the atoms due to electron overlap and Pauli exclusion repulsion between electronic shells. Atoms with $d$-shell valence electronic bands are found to favor the softness of the interatomic potentials compared with those with outer s-shell band ${ }^{53}$. 


\subsection{General features of the glassy state}

Although from the structural point of view a glass does not differ much from a liquid, it is on the dynamical properties where the main differences between a glass and a liquid arise. This subsection aims to provide a short overview of some of the signatures of the glassy state. In order to measure how quickly correlations decay within a system, a correlation function $C(t)$ can be defined as follows ${ }^{40}$ :

$$
C(t)=\frac{1}{N} \sum_{K=1}^{N}\left\langle\varphi_{k}(t) \varphi_{k}(0)\right\rangle
$$

In Eq (2.6) $\varphi_{k}(t)$ represents a generic quantity relative to the particle $\mathrm{k}$, at a given time $t$ and $\mathrm{N}$ denotes the number of particles in the system. A typical choice for $\varphi_{k}(t)$ in experiments is the Fourier transform of density fluctuations. At high temperatures, and after a short transient ballistic regime, $C(t)$ is expected to represent a dissipative regime with exponential relaxation.

$$
C(t)=C_{o} \exp \left(-\frac{t}{\tau}\right)
$$

As the liquid approaches the glass transition, not only the relaxation time $\tau$ increases, but also the shape of $C(t)$ changes qualitatively, as it is shown in Figure $2.3(\mathrm{a})^{55,56}$. Figure 2.3 (a) shows the selfintermediate scattering function for a glass obtained by numerical simulations ${ }^{38}$. It can be seen there that as the temperature is decreased, the function decays by means of a two-step relaxation process. First a fast relaxation takes place, followed by a plateau, and finally a non-exponential decay occurs. Typically, these two processes are known as $\beta$ and $\alpha$ relaxation. The separation of $\beta$ and $\alpha$ relaxation can also be seen from dielectric experiments in which the temperature dependence of the peak relaxation frequency is measured ${ }^{57}$. Figure $2.3(b)$ shows that the relaxation peak splits into slow $\alpha$ and fast $\beta$ relaxations as $\mathrm{T}_{\mathrm{g}}$ is approached ${ }^{38}$. 

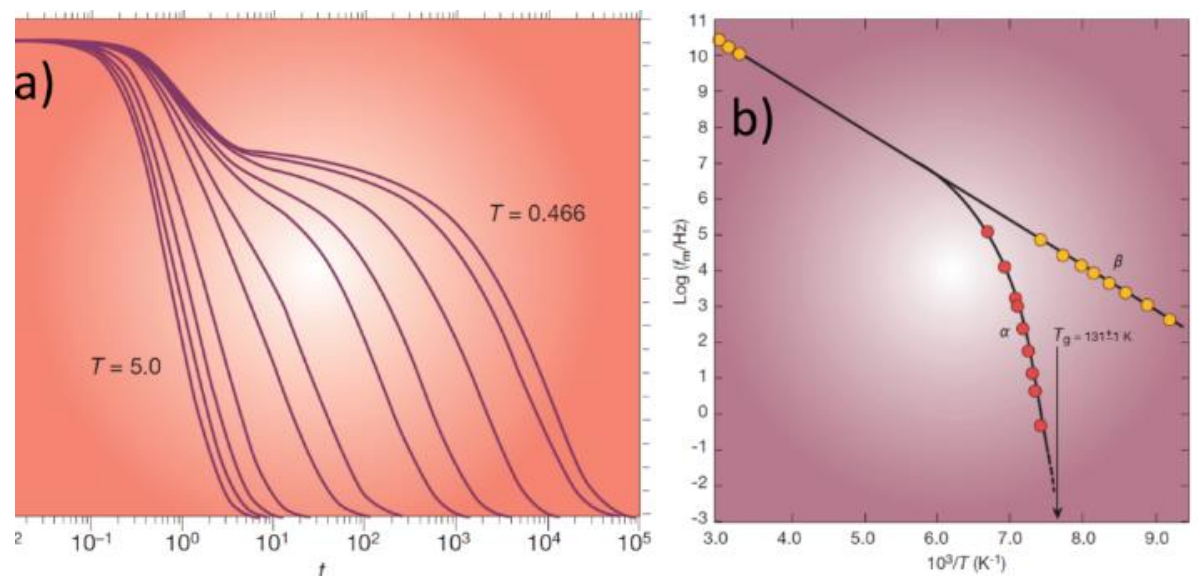

Figure 2.3 (a) Self-intermediate scattering function as a function of time for different temperatures calculated from simulations in Lennard-Jones glass. (b) Temperature dependence of the peak dielectric relaxation frequency of a glass.former ${ }^{38}$

The onset of both relaxation processes can be further investigated with the help of another variable, the Mean Squared Displacement MSD of a given particle $i$, given by the expression:

$$
\left\langle r^{2}(t)\right\rangle=\frac{1}{N} \sum_{i}\left\langle\left\|\vec{x}_{i}(t)-\vec{x}_{i}(0)\right\|^{2}\right\rangle
$$

Figure 2.4, shows the evolution of MSD as a function of time for different temperatures calculated numerically in a Lennard-Jones system ${ }^{56}$.

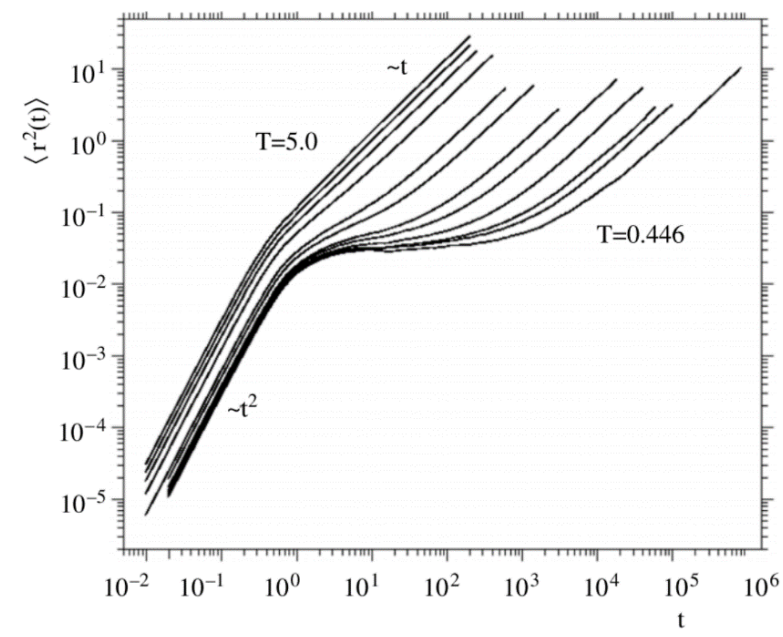

Figure 2.4 Mean-Square-Displacement (MSD) as a function of the logarithm of time in a Lennard-Jones system ${ }^{56}$

At temperatures higher that the glass transition, MSD shows a first ballistic regime where $\left\langle r^{2}(t)\right\rangle \sim t^{2}$ and collisions are negligible, followed by a diffuse regime, where $\left\langle r^{2}(t)\right\rangle \sim t$ and collisions dominate. However, as the temperature approaches $\mathrm{T}_{\mathrm{g}}$, both regimes are separated by a 
plateau. Such plateau represents the cage-effect of the neighboring atoms that prevents particle diffusion. The slow $\alpha$-process corresponds to the escape from the cage, while the fast $\beta$-processes are associated to vibrations confined in the cage ${ }^{56}$.

It has been noticed that the $\alpha$-relaxation shown in Figure 2.3 (a) is non-exponential. In fact, such decay can be successfully fitted with a Kohlrausch-Williams-Watts (KWW) stretched exponential function ${ }^{58}$ :

$$
C(t)=C_{0} \exp \left[-(t / \tau)^{\beta}\right]
$$

The exponent $\beta$ is always $\beta<1$. At temperatures $\mathrm{T} \gg \mathrm{T}_{\mathrm{g}}, \beta$ approaches unity and therefore the KWW function (Equation (2.9)) give rise to an exponential decay. At that temperatures, the plateau also disappears and the whole relaxation process is a simply exponential decay. On the other hand, at temperatures approaching $\mathrm{T}_{\mathrm{g}}, \beta$ decreases with the temperature, thus producing a higher deviation from the exponential decay.

Two different hypotheses have been proposed to explain the non-exponential decay. The first one, often called homogeneous explanation, is that the relaxation takes place non-exponentially all over the glass, so the non-exponential behavior is an intrinsic local property of the glass. The second option, referred as heterogeneous assumption, proposes that relaxation takes place exponentially but with different exponents at different regions. Thus, when the global relaxation time is measured, the spatial average of the different exponential decays would produce the stretched exponential. Global non-exponential behavior of metallic supercooled liquids is believed to arise mainly from a heterogeneous distribution of exponential relaxations ${ }^{59,60}$.

Heterogeneous dynamics are also the source of another feature of supercooled liquids approaching the glass transition, namely the Stoke-Einstein violation. The Stoke-Einstein (SE) relation, provides a relation between diffusion coefficient $D$ and the viscosity $\eta$ :

$$
D \sim \frac{T}{\eta}
$$

This relation does not hold close to the glass transition since $D$ can be much larger than $\frac{T}{\eta}$. The reason for this phenomenon is that the liquid divides into fast and slow regions, often called liquid and solid-like regions, as it approaches the glass transition. In such scenario, diffusivity is mainly determined by the liquid-like region, whereas the main contribution for the viscosity comes from the solid-like clusters ${ }^{38}$. Further evidence of heterogeneities in glasses was given by measurements of the local indentation modulus by Acoustic Atomic Force Microsocopy. Figure 2.5 depicts the map of the local contact resonance frequency for amorphous PdCuSi and crystalline (100)- $\mathrm{SrTiO}_{2}$. Such maps show that the glass exhibit a much wider distribution of local modulus than the crystal ${ }^{61}$. 
a

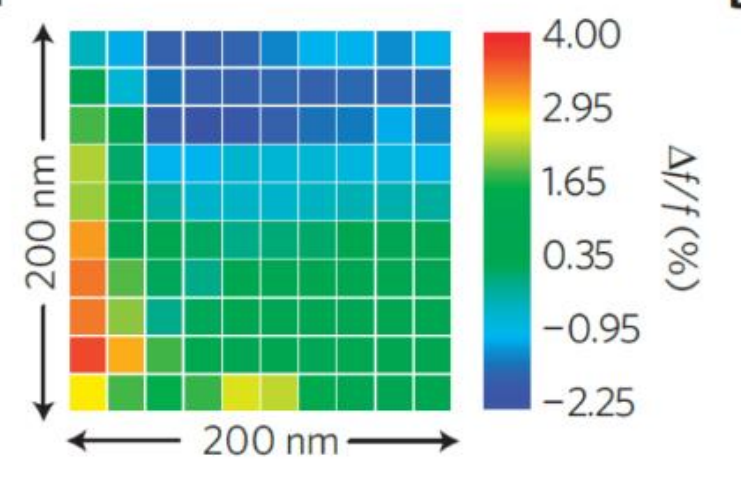

b

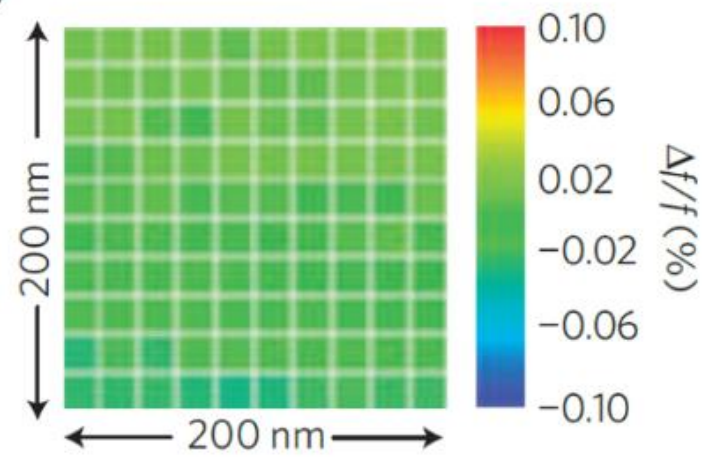

Figure 2.5 Map of the local contact resonance frequencies of (a) PdCuSi glass and (b) (100)-SrTiO crystal $^{61}$. 


\subsection{The Potential Energy Landscape}

In 1969 Goldstein proposed a topographic framework to interpret the metastable dynamics of glasses and supercooled liquids ${ }^{21}$. Such paradigm, that has been adopted and extended by many authors ever since, is commonly known as the Potential Energy Landscape (PEL) ${ }^{62,63}$.

The Potential Energy Landscape is the potential energy function of a system composed by $\mathrm{N}$ constituents: $\phi\left(r_{1} \ldots r_{N}\right)$, where $r_{i}$ includes the position, orientation and vibration of the constituents. For notational simplicity, the multidimensional surface $\phi\left(r_{1} \ldots r_{N}\right)$ is often represented as a function of a generalized coordinate that includes all the degrees of freedom $\left(r_{1} \ldots r_{N}\right)$.
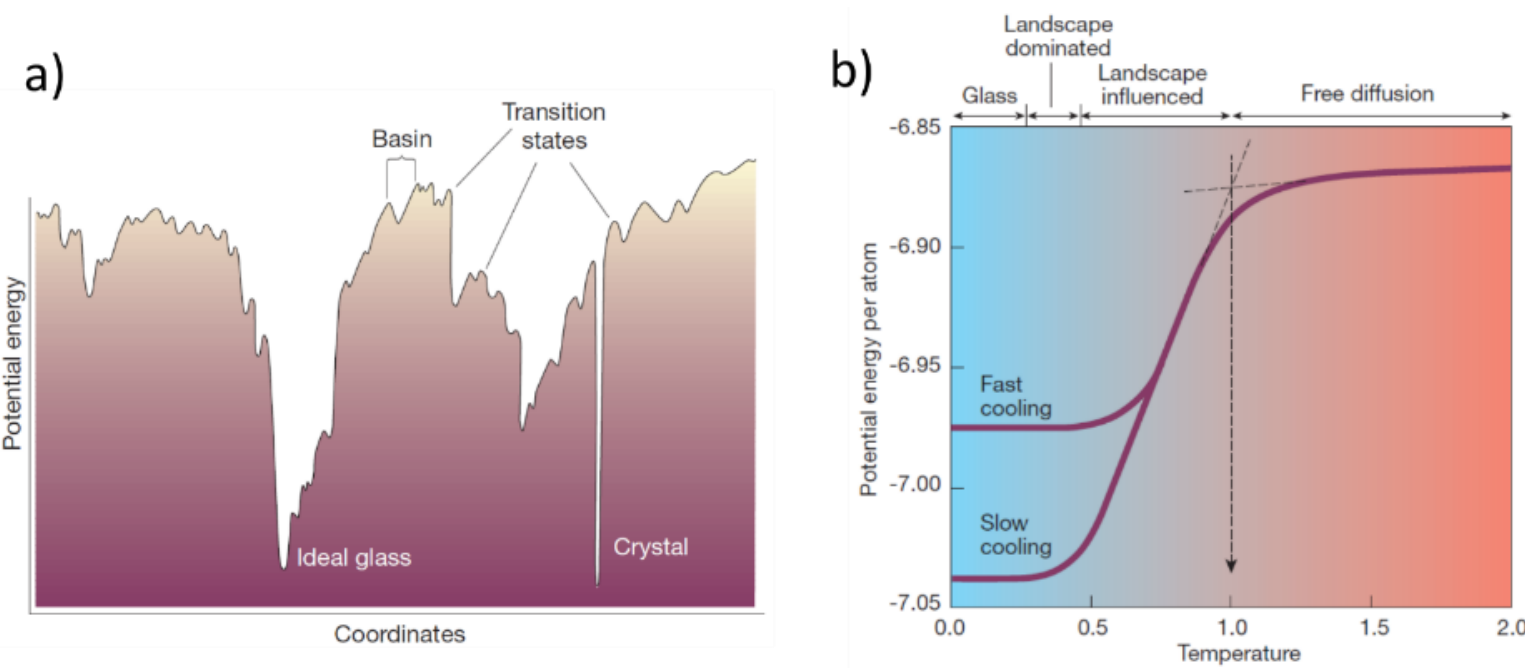

Figure 2.6 (a) Schematic illustration of a Potential Energy Landscape of a glass. (b) Mean energy per particle of a Lennard-Jones glass as a function of temperature ${ }^{38}$.

Figure 2.6 (a) shows an example of the PEL of a glass. The absolute minimum corresponds to the thermodynamic equilibrium position at temperatures lower than the melting point. Such minimum is therefore associated to the crystalline structure. The rest of the PEL is composed by local minima, known as Inherent States (IS's). Such IS's are separated by potential energy barriers, and they share a saddle point (the top of the barrier) which is referred as transient state ${ }^{38}$.

The glass transition can be understood in terms of the ergodicity in the exploration of the PEL. Figure 2.6 (b) shows the potential energy per atom as a function of the temperature computed by means of molecular Dynamics Simulation in a Lennard-Jones glass ${ }^{38}$. For high temperatures, the average potential energy reaches a plateau. That implies that the kinetic energy is higher than the highest energy barrier in the PEL, and the system diffuses freely through the PEL. As the temperature is reduced some energy walls become inaccessible, and the system is confined in certain regions of the PEL. If the cooling rate is low enough, the system will have time to access the minimum corresponding to the thermodynamic equilibrium and crystallize. However, at high cooling rates the configuration will be trapped in a IS corresponding to a local minimum. The depth of such IS would be inversely proportional to the cooling rate, which in turn governs the rate of ergodicity reduction during quenching. As shown in Figure 2.3 (a), in terms of relaxation dynamics 
the free diffusion regime would correspond to the simple exponential decay, whereas as soon as the PEL topography becomes relevant, the relaxation would result into a stretched exponential described by the KWW function.

At temperatures low enough for the system to be trapped in a local minima, transitions between IS's can be described by simple Transition State Theory ${ }^{64}$. According to that theory, the transition rate is given by the following expression:

$$
\dot{\phi}=\phi_{0} \exp \left(-\frac{E}{K_{B} T}\right)
$$

Where $\dot{\phi}$ represent the transition rate, $E$ the energy barrier and $K_{B}$ the Boltzmann constant. In this context $\alpha$-processes has been associated to transitions between neighboring basins, and $\beta$ relaxations to transitions between the fine structure (sub-basins) inside a certain basin, as depicted in Figure $2.7^{63}$. Within the PEL framework, the Shear Modulus $G$ is proportional to the second derivative of the potential energy with respect to the strain $\frac{d^{2} \phi}{d \varepsilon^{2}}$. Considering the aforementioned relation between shear modulus and the curvature of the PEL, a linear relationship between the shear modulus and energy barriers can be written as follows 22,64 :

$$
E=\left(\frac{8}{\pi^{2}}\right) \gamma_{c}^{2} G \Omega
$$

where $\gamma_{c}$ represents the critical shear strain limit, which was shown by Samwer and Johnson to be proportional to $\left(\frac{T}{T_{g}}\right)^{\frac{2}{3}}$ in metallic glasses ${ }^{22}$, and $\Omega$ is the effective STZ volume.

Given the relation between energy barriers and local shear modulus shown in Equation 2.12, heterogeneous mechanical properties of glasses ${ }^{61}$ manifest themselves as a wide distribution of energy barriers in the PEL. Recent theoretical works, demonstrate that such distribution broadens under stress and temperature driving ${ }^{65,66}$. Such heterogeneities have also been associated to the storage of enthalpy and mechanical softening ${ }^{67}$. In this context, T. Dziuba experimentally showed that crystals, ultrastable glasses and normal glasses exhibit a narrowing width of their elastic hetereogeneities distribution. Such difference results in crystals exhibiting a narrow distribution of elastic properties, normal glasses a much greater dispersity, and ultrastable glasses a moderate dispersion in a middle point between crystals and standard glasses ${ }^{68}$. 


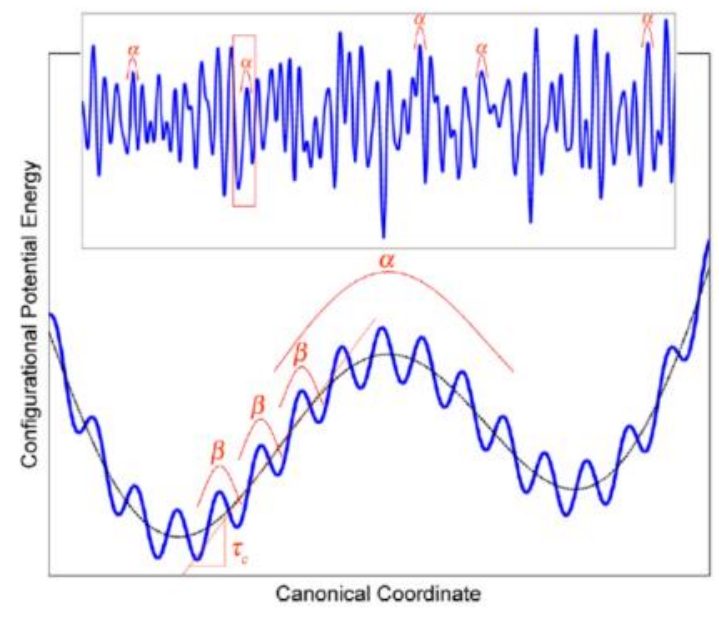

Figure 2.7 Schematic section of a PEL, in which the anelastic reversible $\beta$-processes and the plastic irreversible $\alpha$-processes are represented. ${ }^{63}$

The fragility of a metallic glass is also apparent in the topology of its PEL. Strong glasses are characterized by Arrhenius behavior with a constant activation energy over all range of temperatures. In terms of the PEL, such behavior implies that the landscape of a strong glass former must be homogeneous, with the same energy barriers sampled at different temperatures. In contrast, fragile glasses with super-Arrhenius behavior exhibit activation energies that increase dramatically (faster than exponentially) as the temperature approaches $\mathrm{T}_{\mathrm{g}}$. Super-Arrhenius scaling translates in terms of PEL in a very heterogeneous landscape.

Figure 2.8 illustrates the topological difference between the PEL of strong and fragile glasses. Strong glass formers show homogeneous landscapes and fragile systems exhibit a much heterogeneous topography with a richer fine structure.
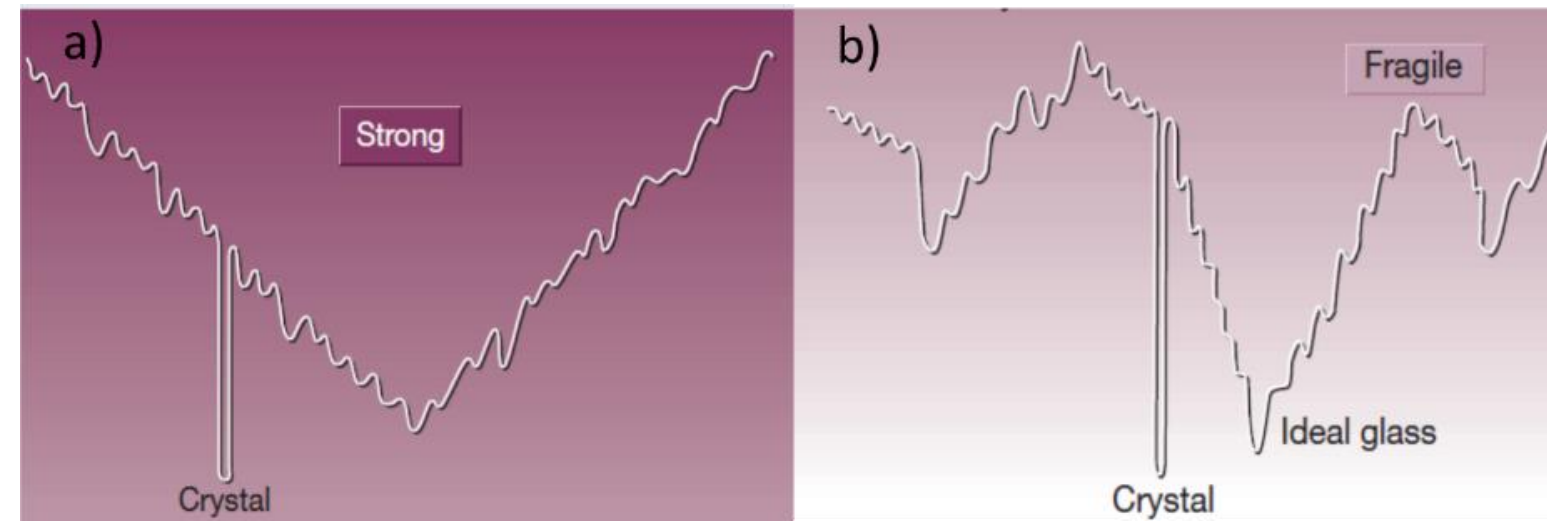

Figure 2.8 Schematic representation of the Potential Energy Landscape of a (a) strong and (b) fragile glass..$^{38}$

Transitions between IS's can be promoted either by mechanical or thermal driving. Such transition can evolve into liquid-like flow if a metallic glass is subjected to mechanical stresses above it yield stress $\sigma_{Y}$, or temperatures above its glass transition $\mathrm{T}_{\mathrm{g}}$. In this context, Molecular Dynamics simulations have shown that liquid-like flow is indeed generated by a coupling of temperature and 
stress ${ }^{69,70}$. It can be seen in Figure 2.9 that yielding, (quantified by the viscosity) can be achieved by means of increasing temperature, stress or a combination of both.

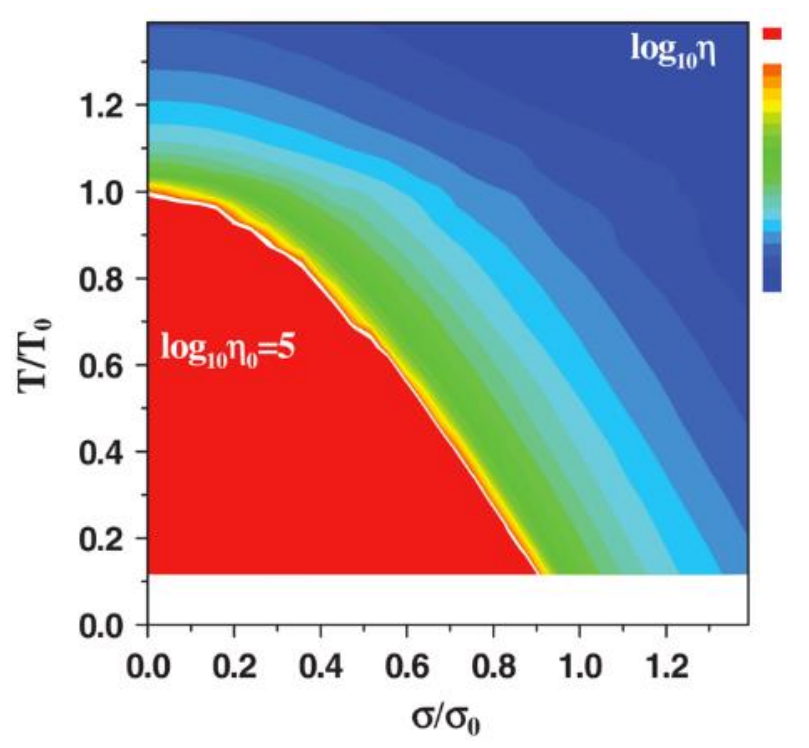

Figure 2.9 Two dimensional plot of the viscosity as a function of normalized stress and temperature calculated by means of molecular dynamics simulations ${ }^{69}$

Experimentally, the effect of stress and temperature on the activation of irreversible plastic events in PdCuSi glasses was estimated by M. Schwabe et al., by means of creep-recovery experiments ${ }^{71}$. It was observed that at temperatures $\frac{T}{T_{g}}<0.9$ both stress and temperature seem to contribute in a similar way to the damping, suggesting that both excitations promote the same kind of structural rearrangements. More recently, Yu et al., studied in detail the microscopical features of strain induced glass transition by means of molecular dynamics simulations ${ }^{72}$. It was observed that in the case of strain-induced yielding, dynamical heterogeneities are reduced, which induces a decrease of the system fragility.

Such difference between temperature and mechanical driving can be understood in the framework of the PEL and taking into account the scalar and vectorial character of temperature and stress respectively ${ }^{73}$. Temperature effectively reduces the height of every barrier in the PEL in an isotropic manner. It results in an increase of the number of accessible states and therefore increases the ergodicity. In contrast, the effect of mechanical stress is to reduce the energy barriers along certain orientation and to increase them along the opposite. Hence, mechanical driving induces an effective tilt and deformation of the PEL. Through this process, some IS's are destroyed and the resulting PEL exhibit a more homogeneous distribution of Energy barriers. Such homogenization effect is the underlying mechanism for the fragile-to-strong transition observed by Yu et al., in strain-driven yielding ${ }^{72}$. 


\subsection{Mechanical properties of metallic glasses}

\subsubsection{General features and deformation modes}

Due to the absence of long range order (LRO), metallic glasses exhibit remarkable mechanical properties compared to their crystalline counterparts 5 . Among them are their high hardness, corrosion and wear resistance ${ }^{10,74}$, high yield strength ${ }^{9}$, and low mechanical damping, but also their limited ductility and thermal instability that lead to embrittlement upon ageing ${ }^{75}$. Figure 2.10 shows the elastic limit $\sigma_{Y}$ as function of the Young modulus $E$ for more than 1500 metals, alloys, composites and metallic glasses. It can be seen there that metallic glasses stand out of the right upper edge of the property map. Metallic glasses present in average $2 \%$ of elastic strain, in contrast to the typical $0.2 \%$ of crystalline materials ${ }^{76}$. Vitrified metals also perform better than crystalline matter in terms of the resilience $\left(\frac{\sigma_{Y}^{2}}{E}\right)$ which quantify the capacity of a given material to store elastic energy ${ }^{9}$. Taken together with their low damping, those features make these materials suitable candidates for practical use in springs, as well as for wave transmission ${ }^{12}$.

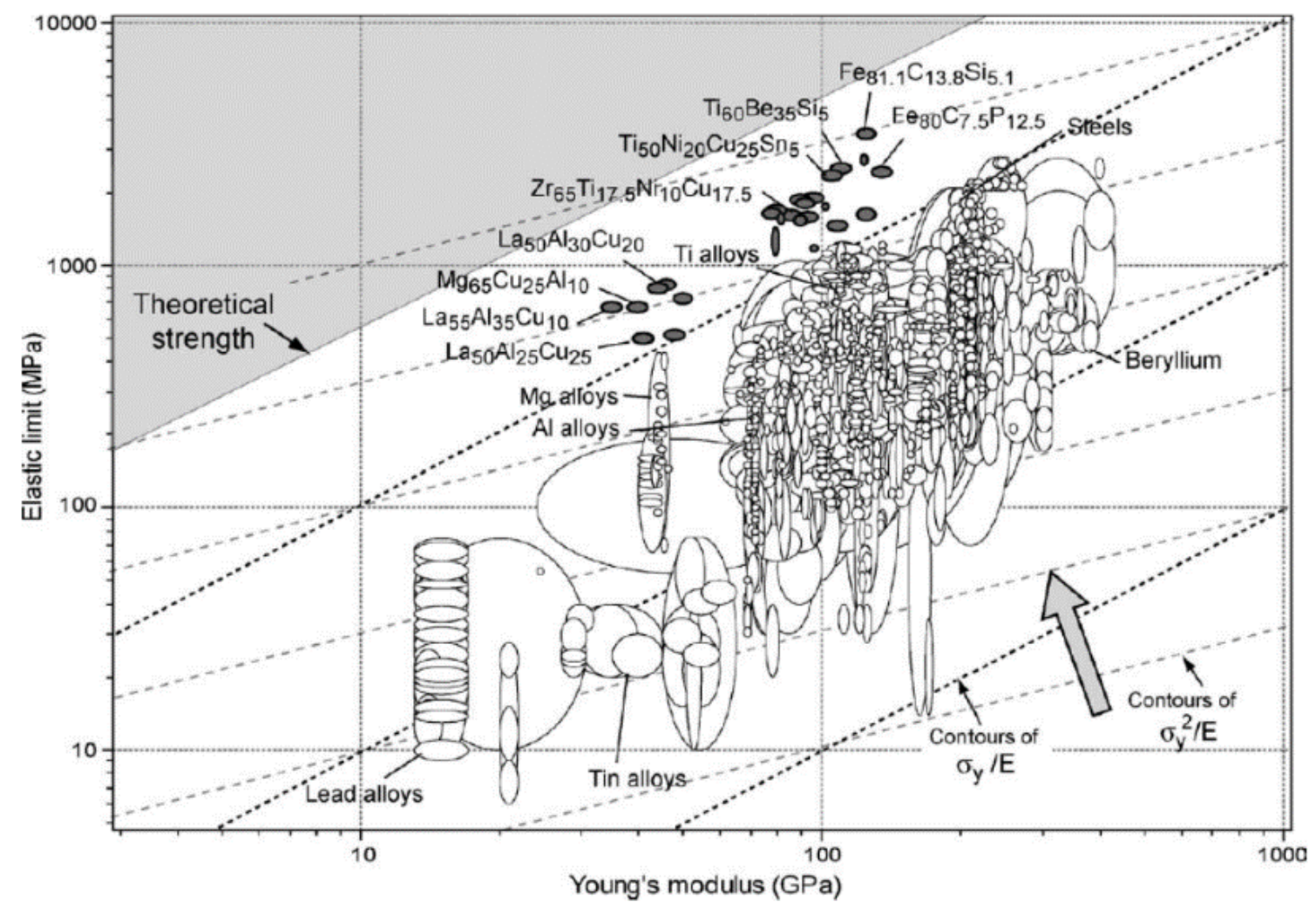

Figure 2.10 Elastic limit and Young modulus for more than 1500 metals, alloys, composites and metallic glasses ${ }^{9}$.

On the other hand, one of the main limitations for the practical use of metallic glasses (apart from their production cost) is their lack of plasticity. At low temperatures, and above the yield stress $\sigma_{Y}$, metallic glasses respond to mechanical load by strain localization in narrow shear bands ${ }^{77,78,79}$. 
Shear band initiation and arrest have been associated to the serrated flow in the plastic region, and eventually lead to crackling and failure ${ }^{80,81,79}$.Many efforts have been devoted to the enhancement of low temperature plasticity of metallic glasses, either by combination with other phases ${ }^{82}$ alloy selection or mechanical treatments ${ }^{83}$. However, the achievement of fair values of ductility, particularly under tension remains elusive and is still one of the main drawbacks for the structural implementation of metallic glasses.

The response to mechanical load of metallic glasses depends dramatically on the experimental conditions, temperature, stress and stress rate. Spaepen summarized for the first time the deformation regimes of vitrified metals in a stress-temperature map ${ }^{77}$, and C.A. Schuh et al. extended that map with recent results and the analysis of the strain rate in their extended review ${ }^{84}$.

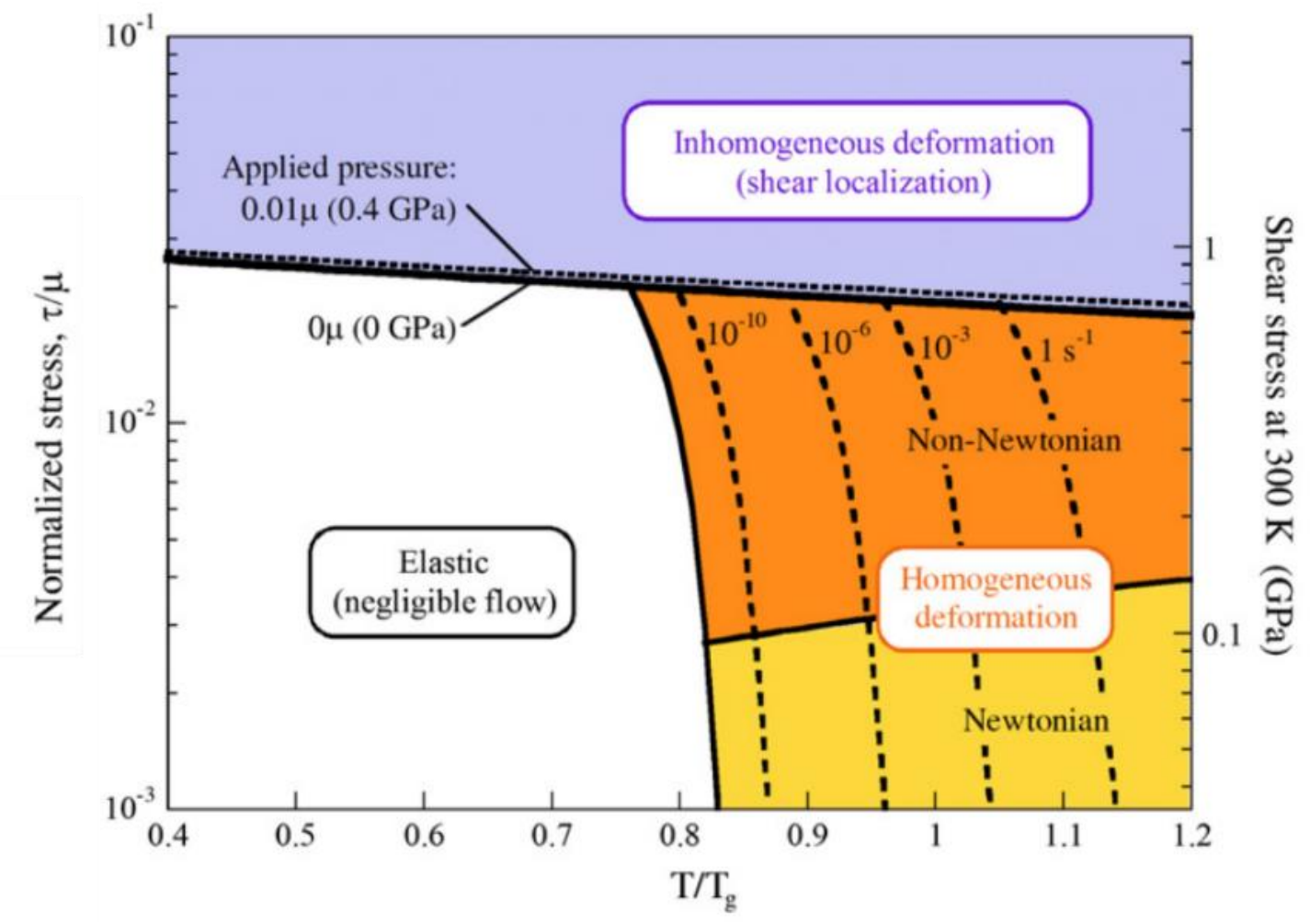

Figure 2.11 Stress-Temperature deformation map for amorphous materials ${ }^{84}$

Figure 2.11 shows the different deformation modes as function of the normalized stress and temperature $\frac{T}{T_{g}}$. At low values of stresses and temperatures the glass does not flow, and the only deformation that takes place is instantaneous and elastic. In the low stress and high temperature regime, the glass flows. At low stresses the flow is Newtonian, characterized by a linear relation between the strain rate and stress $(\dot{\varepsilon} \sim \sigma)$, whereas at high temperatures and moderate higher stresses the system switches to non-newtonian flow, where the dependence of $\dot{\varepsilon}$ on $\sigma$ is no longer linear. Last, at high enough stresses and low temperatures, glass deformation occur via inhomogeneous flow by means of the shear banding phenomenon. 


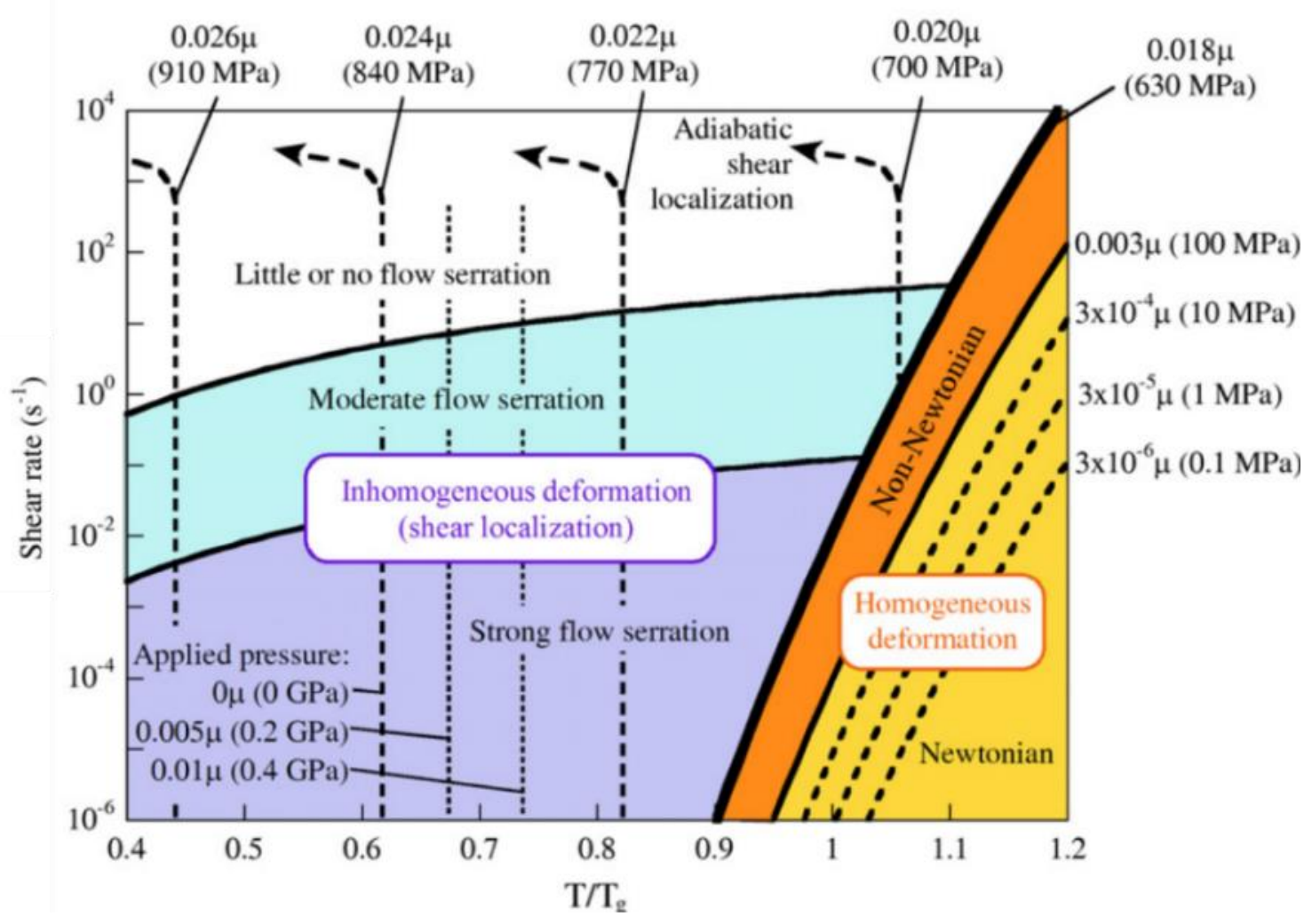

Figure 2.12 Shear rate-Temperature deformation map for amorphous materials ${ }^{84}$

Figure 2.12 illustrates the influence of the shear rate on the deformation mode. The strain rate selects the kind of relaxation processes that can take place, and together with the damping, determines the existence inertial effects. In the high temperature regime, the glass would only flow if it is mechanically loaded at moderate rates. Whether the flow is Newtonian or non-Newtonian depends also in the ratio of strain rate and temperature. Low temperatures and high rates promote non-newtonian flow. In contrast, high temperatures and low rates induce Newtonian flow. In the low temperature regime the shear rate determines the size of the serrations in the serration flow, which are proportional to the energy released through shear banding ${ }^{79}$. Low rates promote big serrations, while high rates reduce the serration size from the stress-strain curve. 


\subsubsection{Microscopic origin of plasticity on metallic glasses}

Deformation modes of crystals are understood in terms of a competition between the different deformation mechanisms (i.e. dislocation climb, glide, diffusional flow..) ${ }^{85}$. In contrast, all modes of deformation described in Figure 2.11 and Figure 2.12 for amorphous metals can be understood in terms of the same microscopic deformation process ${ }^{84}$. Some theories postulated that, at the microscopic level, plasticity takes place by diffusive jumps of individual atoms ${ }^{86,77}$. However, experimenta $\left.\right|^{87}$ and theoretica ${ }^{88,89,90}$ evidence suggests show that plasticity in amorphous systems can be better described through the Shear Transformation Zone or STZ process. The STZ operation was postulated by Argon in 1979 to describe plasticity in amorphous solids ${ }^{20}$. In this picture, the stress is accommodated by a plastic rearrangement of clusters of about 100 atoms ${ }^{91}$, often called soft spots or STZs, embedded in the amorphous matrix. The triggering of a STZs propagates an Eshelby stress field ${ }^{92}$ with quadrupolar symmetry, which induces an effective softening and hardening of the amorphous matrix along certain directions. Contrary to the case of dislocations in crystals, STZs are not structural defects, and can only be defined by it transience or triggering ${ }^{93}$. However, the location for the triggering of potential STZs is believed to be affected by the local properties of the glassy matrix. In this context, Falk recently pointed out that the local yield stress seems to be the best parameter for prediction of potential STZ sizes ${ }^{94}$. Figure 2.13 shows an STZ pattern experimentally observed in a colloidal glass ${ }^{87}$. The color code represents the non-affine displacement of the atoms, which is the difference between the total displacement and the displacement that would be expected from a purely elastic relation between the strain and stress. A central plastic core can be distinguished, as well as a quadrupolar shaped strain displacement field, in good agreement with theoretical predictions ${ }^{95,96}$.

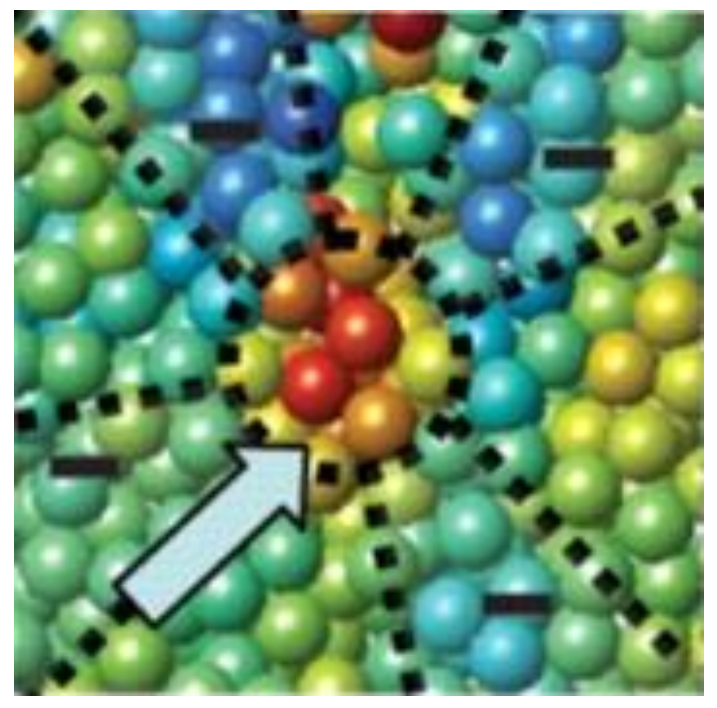

Figure 2.13 Shear Transformation Zone observed in a sheared colloidal glass by means of confocal microscopy ${ }^{87}$

The triggering of STZs may take place independently in the glassy matrix or coupled with neighboring STZs along certain orientations. The resulting process is a consequence of the competition between a random distribution of potential sites in the whole volume of the glassy matrix and the ability of the STZs stress field to induce a soft spot in the vicinity of an already 
triggered STZ. Such phenomenology has been numerically analyzed by E.R. Homer et al. by Monte Carlo Simulations ${ }^{25}$. Figure 2.14 shows a deformation-map which depicts the STZ cooperativity as a function of the stress and temperature.

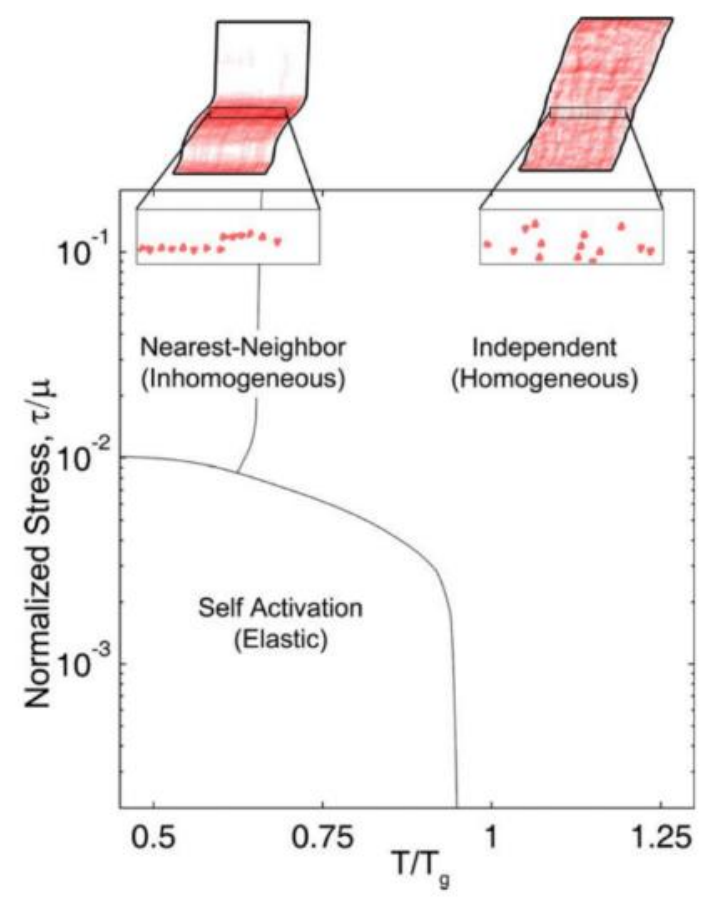

Figure 2.14 Stress-temperature map of the STZ cooperativity calculated from kinetic Montecarlo simulations ${ }^{25}$

Figure 2.14 shows three distinct zones in the deformation map. At low stresses and temperatures, the glass deforms elastically, which is associated to the so-called STZs self-activation. In fact, the probability of the same STZs to be back-triggered upon unloading within the elastic region is negligible, but the elastic region is characterized by the ability of the amorphous matrix to accommodate stress without percolation of STZs. At low temperatures and high stresses, the STZs performs nearest-neighbor activation. That means that the quadrupolar interactions play a dominant role and the activation of the STZ is mostly determined by the location of previous ones and their effect in the local mechanical properties. Such cooperativity of STZs, which may take place in the form of avalanches of $\mathrm{STZs}^{30}$ is believed to be in the origin of the strain localization and shear banding. Finally, the homogeneous flow that takes place at high temperatures is generated by independent activation of STZs across the whole volume of the material or by coupling through the whole system. In such scenario STZs triggering can be modelled as an independent process. However, the way in which the dynamics among STZs take place remains unclear, and there is hope that the statistical analysis of the intermittent deformation signal generated by the avalanches may shed some light in this problem. The field of the avalanche dynamics and its application to the study of plasticity in amorphous matter is introduced in section 2.6 


\subsection{Magnetic properties of metallic glasses}

\subsubsection{Structural disorder and magnetic order}

Magnetism in the solid state is one of the most studied topics in the field of physics ${ }^{14}$. Therefore, there is a huge variety of theories and models to understand the wide range of phenomenology in this matter ${ }^{14,97,98}$. However, from a simplified point of view, there are two main approaches to analyze magnetic order. Magnetism could be modelled by the presence of localized magnetic moments which interact through exchange and dipolar interaction, or could be thought of as the exchange splitting of delocalized spin polarized electronic wave functions ${ }^{14}$. The former approach is suitable to understand magnetism coming from unpaired $4 \mathrm{f}$ electrons, which is the case for rare earth alloys. In such situation, the $4 \mathrm{f}$ shell is highly localized and well shielded by outer electrons, thus yielding to vanishing overlap integrals with neighbor atoms. The latter approach is best suited for the magnetism of transition metals (TM), like Fe, Co, Ni, whose features arise from highly delocalized d-orbitals, which overlap with other bands, either from the same atom or from other ligands. In this case, according to the Stoner Criterion, the wave function becomes spin polarized if the following condition is fulfilled:

$$
I D\left(E_{F}\right)>0
$$

Where $I$ is the stoner exchange parameter and $D\left(E_{F}\right)$ represents the density of states at the Fermi level.

The experiments on magnetic samples through this work were performed in Co and Fe rich alloys. Therefore, in the following only magnetism coming from delocalized $3 \mathrm{~d}$ bans is considered.

The wave function of the TM is partially mixed due to interaction with the ligands present in the alloy. Such mixing depends on the overlap integrals which in turn depend on the interatomic distance, among other parameters. In addition, the environment of a given atom produces an electrostatic field that also interacts with the magnetic orbital through spin orbit interaction. Due to the lattice symmetry, in crystalline materials such electrostatic field is homogeneous and known as crystalline field, which is the source of the global magneto-crystalline anisotropy ${ }^{14}$.

The main consequence of the amorphous state in TM-based metallic glasses is that both the crystal field and the overlap integrals are not constant anymore but rather depend of the local environment of each site. The distribution of overlap integrals give rise to a distribution of different occupation of the magnetic orbitals at different sites. Besides, the crystal field is substituted by local anisotropy fields with a distribution of preferred directions that varies from site to site according to the local environment. In the case of $3 \mathrm{~d}$-glasses the exchange correlation length is larger than the characteristic length of the local anisotropy, so the latter is averaged out ${ }^{99,100}$.

The lack of magneto-crystalline anisotropy, and the absence of structural defects or grain boundaries in which magnetic walls may be pinned, is the cause of the excellent soft behavior of 
$3 \mathrm{~d}$ based magnetic metallic glasses. These two factors favor their easy magnetization, either by wall motion or rotation processes.

A final remark for this subsection is that in words of O'Handley: "magnetism, even in $3 \mathrm{~d}$ alloys, is predominantly a local phenomenon determined by the immediate environment about potentially magnetic atoms ${ }^{\prime 101}$. The importance of the local environment is therefore a common characteristic of plasticity and magnetism in metallic glasses.

\subsubsection{Magnetostriction: Local origin and macroscopic effect}

The term magnetostriction was defined as the change in dimensions of a magnetic material when subjected to an external bias field ${ }^{102}$.Such coupling between the magnetic and elastic properties of the solid arises from the dependence of the anisotropy energy on the lattice strain. A common parameter to quantify the magentostriction of a material is the saturation magnetostriction constant $\lambda_{s}$, which is defined as follows:

$$
\lambda_{s}=\frac{L_{s}-L_{D}}{L_{D}}
$$

where $L_{S}$ and $L_{D}$ represent the length of the sample in the magnetically saturated and demagnetized state respectively. $\lambda_{s}$ ranges from -10 to $+30 \times 10^{-6} 101$

Another parameter to characterize magnetoelastic effects is the so called magnetoelastic coupling coefficient $K^{2}$, which represents the transference ratio between the magnetic and mechanical energy, and is such that $K^{2}<1^{103}$. A review of different experimental techniques to measure $K^{2}$ is given in ${ }^{104}$, which includes resonance and Young modulus measurements.

There are two main microscopic sources of magnetostriction, which are usually referred as one-ion, or local field contribution and two-ion or anisotropic exchange contribution ${ }^{105,106,99}$. The single ion component of the magnetostriction is associated to the deformation of the local units or short range order around the magnetic atom ${ }^{106}$.The coupling is originated by the spin orbit interaction, which couples the magnetization direction to the distortion of the surrounding local structure through the electron orbitals. The two-ion component is associated to the rigid rotation of these local units, which can reduce their energy by their reorientation which respect to the direction of magnetization ${ }^{107}$. According to $\mathrm{O}^{\prime}$ Handley the one-ion term should be proportional to the third power of the saturation magnetization, whereas the two-ion term should follow a quadratic relation with the saturation magnetization ${ }^{101}$. Therefore, the total magnetostriction can be written

$$
\lambda_{S}(T)=\alpha\left[M_{S}(T)\right]^{3}+\beta\left[M_{S}(T)\right]^{2}
$$

where $\alpha, \beta$ are proportionality constants and $M_{S}$ is the saturation magnetization. Experiments on Fe-rich alloys suggest that the main contribution of magnetostriction in that case is the one-ion mechanism. In Co-rich alloys the experimental results can only be explained by a combination of 
both mechanisms as depicted in Eq (2.14). Competition of both mechanisms is the origin of the vanishing magnetostriction in certain alloys of $\mathrm{Fe}$ and $\mathrm{Co}^{108,109}$. Figure 2.15 shows a triangular map, which displays the magnetostriction $\lambda_{s}$ for for $\mathrm{Fe}, \mathrm{Co}$ and Ni alloys of different composition. It can be seen that Fe-rich alloys exhibit high and positive $\lambda_{s}$, whereas Co-rich composition show low negative $\lambda_{s}$. The solid line indicates the composition of vanishing magnetostriction.

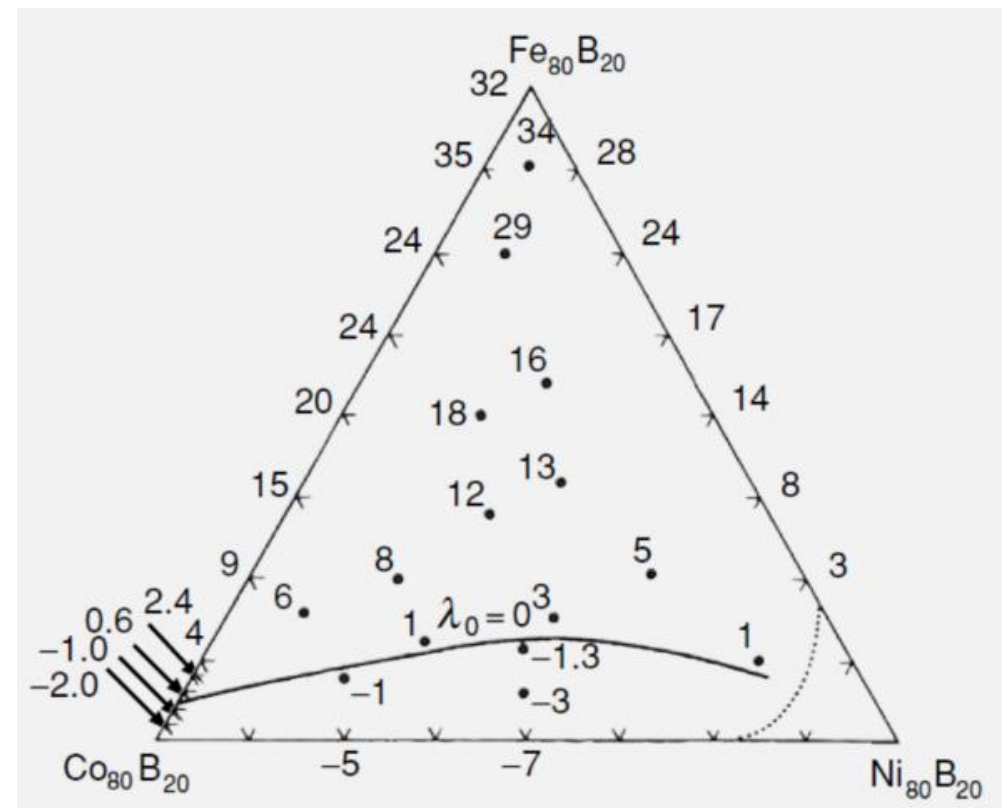

Figure 2.15 Triangular Map of magnetostriction values as a function of the element composition for $\mathrm{Fe}, \mathrm{Co}$ and $\mathrm{Ni}$ alloy.The solid line indicates the composition with vanishing magnetostriction ${ }^{14}$

One of the main macroscopic effects of magnetostriction is the so-called $\Delta E$-effect ${ }^{14,103} . \Delta E$-effect is the difference between the young modulus measured with and without an external magnetic bias field, and is represented in a normalized form, as follows

$$
\frac{\Delta E}{E}=\frac{E-E_{H}}{E}
$$

Where $E, E_{H}$ represent the young modulus measured with and without an applied field. In some metallic glasses $\Delta E$ can reach values $\Delta E \sim 0.9$

The origin of the $\Delta E$-effect is the anisotropy induced by the magnetoelastic coupling. Due to this anisotropy, an extra strain $\lambda_{S}$ is exerted by the magnetic domains along their direction of magnetization. If those domains rotate towards the direction of deformation during the deformation process, the sample exhibits and extra strain $\lambda_{S}$ on top of the normal elastic strain $\frac{\sigma}{E}$. Such extra strain is experimentally measured as a reduction of the Young modulus, which is the $\Delta E$ effect $^{110}$. Livingston showed analytically that the magnetomechanical coupling, and therefore the 
$\Delta E$-effect is maximized when the applied field equals the anisotropy field of the magnetostrictive sample ${ }^{103}$.

Figure (2.16) shows the $\Delta E$-effect in a $\mathrm{Fe}_{40} \mathrm{Ni}_{40} \mathrm{P}_{14} \mathrm{~B}_{6}$ glass with two different domain patterns, parallel $\left(E_{\|}\right)$and perpendicular $\left(E_{\perp}\right)$ to the ribbon longitudinal axes. In both cases the mechanical stress and magnetic field are applied along the longitudinal axes. It can be seen that a significant reduction of the Young modulus ( $\Delta E$-effect) only take place in the $\boldsymbol{E}_{\perp}$ case, since there is a rotation of the domains towards the direction of deformation. No further modulus reduction take place for high field intensities since the magnetization is saturated.

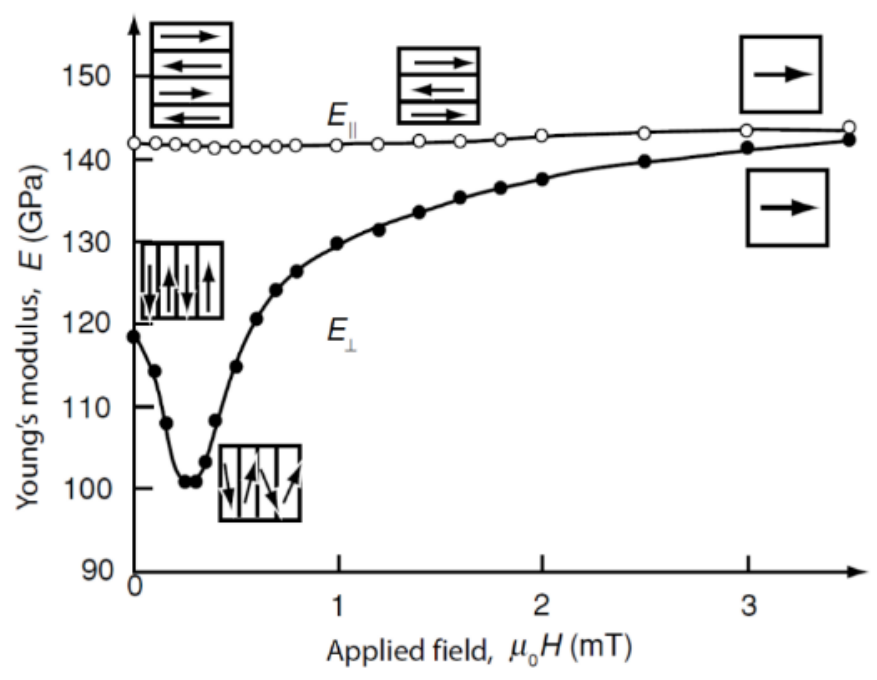

Figure 2.16 Young modulus as a function of magnetic field intensity for a metallic glass with magnetic domains parallel $\left(\boldsymbol{E}_{\|}\right)$and perpendicular $\left(\boldsymbol{E}_{\perp}\right)$ to the applied bias field ${ }^{14}$

Magnetostriction is a key parameter for the industrial application of magnetic metallic glasses. Materials with vanishing magnetostriction are suitable for applications in which their soft magnetic behavior need to remain insensitive to mechanical stresses, as is the case of transformers. On the other hand, magnetostrictive glasses are of great use for sensing applications ${ }^{17,18,19}$.The coupling between the mechanical and magnetic properties allows the wireless operation of metallic glassesbased devices, which have been implemented in density ${ }^{111}$, viscosity ${ }^{112}$, stress ${ }^{113}$ and biological ${ }^{114}$ sensors. 


\subsection{Crackling noise, avalanche dynamics and intermittency}

A system is said to crackle when it responds to a smooth load with intermittent discrete events of a wide distribution of sizes ${ }^{33,34}$. Many physical systems in nature exhibit crackling behavior, including Earth tectonic plate dynamics ${ }^{115}$ ( whose manifestation are the feared earthquakes), snow ${ }^{116}$, or sand ${ }^{117}$ avalanches, friction in surfaces ${ }^{118}$, domain wall motion in magnetic materials ${ }^{119,120}$ (known as Barkhausen Noise), materials undergoing martensitic transformations ${ }^{121}$, superconductor dynamics ${ }^{122}$ and turbulence in plasma flow ${ }^{123}$ among others.

Strikingly, phenomenology associated to crackling noise has also been observed in many nonphysical systems, as diverse as fluctuations in stock markets ${ }^{124}$, decision making problems ${ }^{125}$, and neuronal networks ${ }^{126}$. This kind of intermittent behavior is then generally referred as avalanche dynamics. The wide range of avalanching systems suggest that those dynamics should not depend on the microscopic details but rather in global features as the topology and interaction among the units of the process under consideration ${ }^{33}$.

Avalanche dynamics were initially associated to critical points in phase transitions. In this context, a paradigmatic model is the Random Field Ising Model (RFIM) ${ }^{127}$. In a simplified version of that model, a magnetic sample is represented as a lattice of domains, with a two state spin variable $S_{i}=$ $-1,+1$ and the force on each domain is given by the following expression:

$$
F_{i}=H(t)+\sum_{j} J S_{j}+h_{i}(R)
$$

Where $H(t)$ represents the external field, J the exchange coupling with $j$ neighboring spins and $h_{i}$ is a local anisotropy field which represents randomness and other kind of disorder modulated by the parameter R. Numerical simulation of RFIM shows that if $\frac{J}{R}>>1$ the system responds to a smooth driving force $H(t)$ by a collective flipping of many spins. As a result, the system responds to a smooth driving with big changes of magnetization (events) only. On the other hand, if $\frac{J}{R} \ll 1$ the randomness suppresses interactions and all spins flip independently. As a consequence, the system responds to the driving force $H(t)$ with many small events. Avalanches with a wide range of values take only place for a certain value of disorder $R_{C}$, which defines a critical point.

RFIM and other theories provide a model for the avalanche phenomenology but do not explain why do avalanches take place in nature. If it would be required to bring a system to a critical point to observe this behavior it should be extremely difficult to obtain experimental confirmation of that behavior. However there is multiple experimental evidence of avalanching systems ${ }^{33}$. To address this issue, Bak Tang and Wiesenfeld proposed the idea of the Self-Organized-Criticality (SOC) ${ }^{128,129}$. According to their theory, in many circumstances real systems tend to self-organize themselves towards a critical point. Therefore, it is not necessary to fine tune experiments to observe this behavior. However, the ultimate reason for this phenomenon is still unknown, and may depend on 
the specific system under study. In this context, recent results in avalanche dynamics in brain tissue, suggest that the assumption of SOC satisfactorily agrees with optimal performance of information transmission and time reaction of the brain ${ }^{130,131}$. 


\subsection{Statistical analysis of avalanches}

The study of avalanching systems is performed by means of the statistical analysis of the avalanches. Generally speaking, an intermittent signal can be divided in avalanches and inter-event times. Relevant avalanche parameters are their size, energy and duration. The wide distribution of events is attributed to self-similarity which is associated to a power law probability distribution of several avalanche parameters. In case of high resolution experiments or simulations, avalanche shapes and velocity profiles can be analyzed, which is believed to provide deeper insights about the underlying mechanisms ${ }^{132}$. Figure 2.17 shows the probability distribution of Barkhausen Noise avalanches sizes produced by amorphous and crystallized samples under different conditions. The data scale with two different exponents, which are $\tau_{1}=-1.5$ (solid line), and $\tau_{1}=-1.27$ (dashed line) that the author associates to different universality classes. ${ }^{133}$

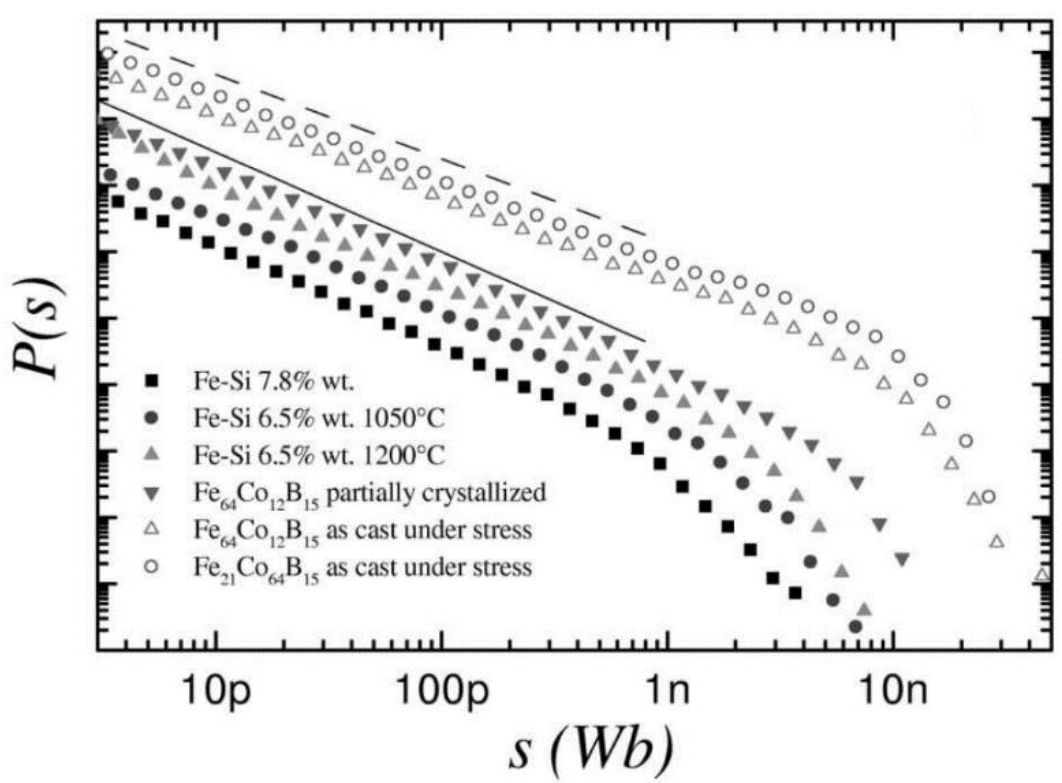

Figure 2.17 Probability distribution of avalanche sizes in Barkhausen Noise experiments for amorphous and crystalline samples ${ }^{133}$.

Avalanching systems can be classified in different universality classes according to their statistical features, which are given by the exponents of the power law distribution among other quantities ${ }^{24}$. The fact that different systems display the same avalanche statistical features suggests that they share dynamical properties, independently of their microscopic details. One example of universality class is the Mean Field (MF) approximation, which assumes that interactions between system constituents can be modelled as the interaction between one of those constituents and an effective field ${ }^{134,135}$. The fact that Earthquakes produce avalanches with analogous statistics as porous samples under compression suggests that, despite the remarkable difference in length scale, both dynamical systems share the same universality class ${ }^{136}$. Table 2.1 provides an overview of the avalanche exponents expected from different models ${ }^{137,138}$. 
Table 2.1 (a) Scaling exponents for different universality classes. (b) Scaling exponents as function of damping $\Gamma$

\begin{tabular}{lccccc}
\hline \hline a) & Expression & EP 2D & EP 3D & $\left(1 / r^{2}\right)$ depinning 1D & MF \\
\hline$\beta$ & $\dot{\gamma} \sim(\Delta \sigma)^{\beta}$ & $1.54(2)$ & $1.55(2)$ & $0.625(5)$ & 2 \\
$\tau$ & $P_{S} \sim S^{-\tau}$ & $1.28(5)$ & $1.25(5)$ & $1.25(5)$ & 1.5 \\
$d_{f}$ & $S_{c} \sim L^{d_{f}}$ & $0.90(7)$ & $1.3(1)$ & $\sim 1.38$ & $\ldots$ \\
$\tau^{\prime}$ & $P_{T} \sim T^{-\tau^{\prime}}$ & $1.41(4)$ & $1.44(4)$ & $\sim 1.43$ & 2 \\
$\alpha$ & $T_{c} \sim \dot{\gamma}^{-\alpha}$ & $0.38(4)$ & $0.30(4)$ & $\ldots$ & $\ldots$ \\
$z$ & $T \sim \ell^{z}$ & $\sim 0.57$ & $\sim 0.82$ & $0.77(1)$ & $\ldots$ \\
$\delta$ & $S \sim T^{\delta}$ & $1.58(7)$ & $1.58(5)$ & $\sim 1.7$ & 2 \\
$\theta$ & $P_{x} \sim x^{\theta}$ & $0.52(3)$ & $0.37(5)$ & 0 & 1 \\
\hline \hline \multicolumn{6}{c}{} \\
b) \\
\hline \hline$\Gamma$ & $d$ & $\tau$ & $\alpha$ & $\gamma$ & $\phi$ \\
\hline 1.0 & 2 & $1.3 \pm 0.1$ & $0.9 \pm 0.05$ & $1.3 \pm 0.1$ & $1.00 \pm 0.1$ \\
0.1 & 2 & $1.0 \pm 0.05$ & $0.8 \pm 0.1$ & $1.2 \pm 0.1$ & $0.9 \pm 0.1$ \\
0.001 & 2 & $1.25 \pm 0.1$ & $1.6 \pm 0.1$ & $0.8 \pm 0.1$ & $0.5 \pm 0.1$ \\
1.0 & 3 & $1.3 \pm 0.1$ & $1.1 \pm 0.1$ & $2.1 \pm 0.1$ & $1.5 \pm 0.2$ \\
0.1 & 3 & $1.05 \pm 0.05$ & $1.5 \pm 0.1$ & $1.6 \pm 0.1$ & $1.30 \pm 0.1$ \\
0.001 & 3 & $1.2 \pm 0.1$ & $2.1 \pm 0.2$ & $1.3 \pm 0.2$ & $0.9 \pm 0.1$ \\
\hline \hline
\end{tabular}

Perfect power laws predicted by avalanche dynamics theory can be deformed or modified due to several reasons. For instance, inertial effects have been shown to produce a deformation of the power law distribution of avalanche sizes and the generation of characteristic hump ${ }^{139,140,141}$. Besides, as shown in stick-slip simulations, the distribution of avalanche sizes departs from a clean power law shape as the system is driven from a subcritical to a supercritical state changing the coupling strength parameter ${ }^{142}$. Figure 2.18 (a) shows the effect of the cooperativity factor $\alpha$ on the probability distribution of avalanche sizes in a stick-slip model simulation. A perfect power law arises for the critical value of cooperativity $\alpha=\alpha_{c}=0.99$. such power law is truncated by a cutoff for subcritical cooperativity $\alpha<\alpha_{c}$, and the probability of small avalanches is reduced in the supercritical state $\alpha>\alpha_{c}{ }^{142}$. Figure 2.18 (b) shows that a characteristic hump appears as a consequence of inertia. Inertial effects are controlled by the damping: underdamped systems exhibit high inertial effects, whereas in overdamped systems the inertia is suppressed. The events can be divided in small power law distributed avalanches (light yellow) and big inertial avalanches with a characteristic length scale (dark yellow) $)^{139}$. 

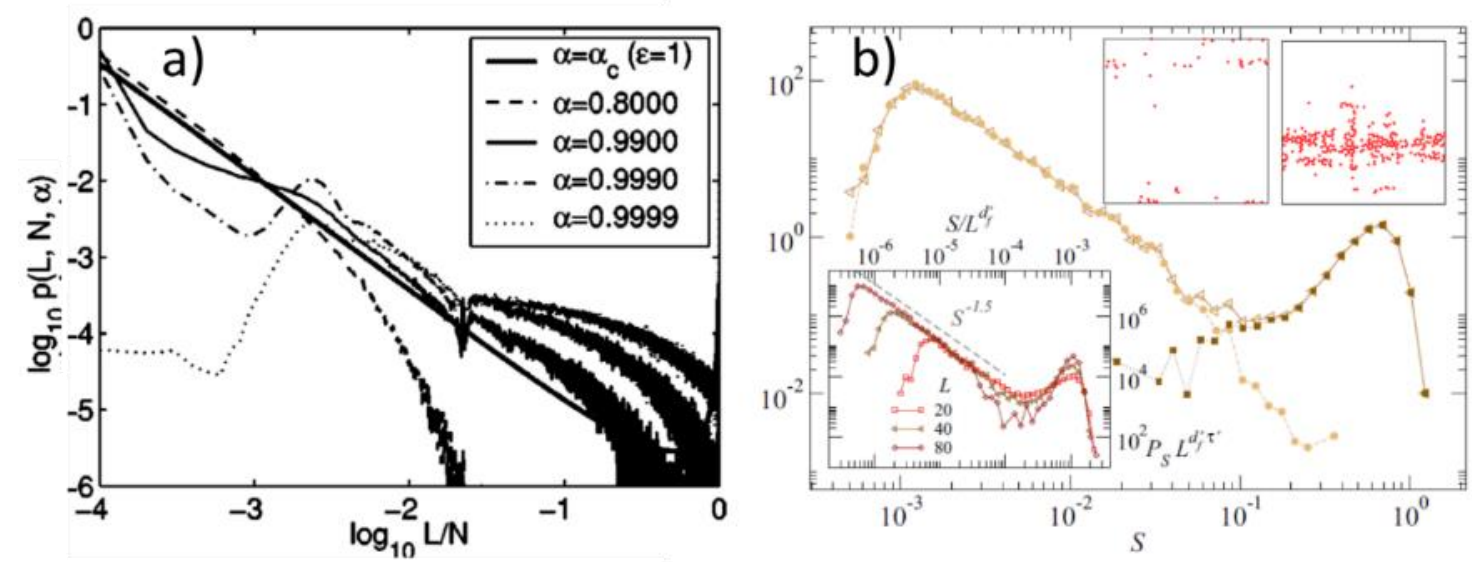

Figure 2.18 (a) Effect of the cooperativity parameter $\alpha$ on the avalanche size probability distribution in a stick-slip model ${ }^{142}$. (b) Inertial effects on the probability distribution of avalanche sizes calculated from an elasto-plastic model. The events are divided in small power law distributed avalanches (light yellow) and big inertial avalanches with a characteristic length scale ${ }^{139}$

A change in the underlying dynamics that govern an avalanching system has an impact on the statistical features of the avalanches, which reflects in a crossover on the scaling exponents. A crossover can take place due to a change in the experimental conditions ${ }^{143,138}$,or due to a transition in the underlying mechanism that govern the process under study ${ }^{144}$.

Finally, as pointed out in a recent work from E.K.H Salje et al. ${ }^{145}$, the measured response of a dynamical system can be the result of a superimposition of several dynamical process, and may separately follow power laws with different exponents. In that case, the resulting signal may display an exotic exponent which would be a result of the mixing of each of the underlying processes. 


\subsection{Crackling Noise in metallic glasses}

Avalanche dynamics and crackling noise have been studied extensively in the context of deformation of materials, both amorphous and crystalline. The first experiments of Crackling Noise produced during deformation, were done by Becker et al., in sheared Zinc crystals in $1932^{146}$. Back then, the authors already associated it with "dislocation avalanches". More recently J. Weiss measured the crackling of different crystals with Acoustic Emission experiments, and differentiated between wild (crackling) noise and mild (gaussian) noise ${ }^{147,148}$. A higher amount of crackling noise was observed in crystals with FCC lattice compared to HCP structures .That difference was associated to long-range interactions and anisotropic glide mechanism which is favoured in HCP structures. In addition the effect of polycrystallinity and grain size was analyzed and its impact resulted to be more complex than simple introduction of a finite size effect ${ }^{149}, 150$.

In the context of amorphous materials, the most obvious intermittent behavior can be found in the serrated flow which is observed when a material is sheared at stresses above the yield stress $\sigma>$ $\sigma_{Y}{ }^{80}$.Some experiments have shown avalanche distribution with exponents compatible with the mean field universality class ${ }^{28,151}$, in agreement with a Mean Field plasticity model ${ }^{31}$. On the other hand, other results obtained by Molecular Dynamics simulations ${ }^{152}$, elasto-plastic ${ }^{24}$, and fully tensorial models ${ }^{153}$, yield critical exponents incompatible with the mean field approach. However, recent results obtained from elasto-plastic models show that the MF exponents are recovered in the limit of high strain rates ${ }^{154}$.

A physical interpretation for the failure of Mean Field models is that it assumes a positively definite elastic kernel, which is not the case for the Eshelby quadrupolar propagator. A STZ favors the trigger of other STZ's along certain directions but hampers it along others. Such asymmetry or anisotropy is at odds with a mean field approach. Recent results in molecular dynamic simulations show that small avalanches take also place in the so-called elastic region of the stress-strain curve ${ }^{152}$. Those avalanches share statistical features with the ones that take place in the plastic region.

Avalanche dynamics concepts have also been applied to the analysis of creep deformation of a $\mathrm{Pd}_{77.5} \mathrm{Cu}_{6} \mathrm{Si}_{16.5}$ metallic glass at high temperatures ${ }^{30}$. Waiting times between resolvable events were analyzed and a crossover was observed in their probability distribution. That crossover was interpreted as a signature of a transition from a regime of 3D activation of STZ's characterized by an exponent $\tau=1.4 \pm 0.1$ to a correlated nano-shear bands characterized by an exponent $\tau=$ $0.8 \pm 0.1$. Figure (2.19) shows the distribution of waiting times split in two regimes with different slopes. 


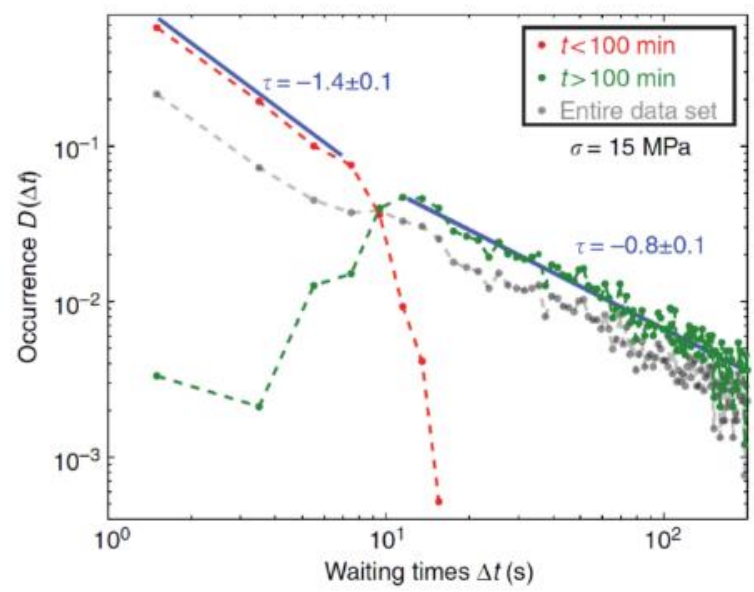

Figure 2.19 Waiting time probability distribution measured during a creep test on $\mathrm{Pd}_{77.5} \mathrm{Cu}_{6} \mathrm{Si}_{16.5}$ at $\sigma=15 \mathrm{MPa}$ and $\mathrm{T}=593 \mathrm{~K}$. The distribution is split into two regimes with different slopes, originating from the first $100 \mathrm{~min}$ of the creep curve and the subsequent part.

The magnetoelastic coupling has been shown to have an influence on the avalanche behavior of metallic glasses. Numerical simulation have shown that the mechanical and magnetic instabilities take place simultaneously in magnetic metallic glasses ${ }^{155}$. Also, measurements of Barkhausen noise under different mechanical stresses showed that magnetic avalanche sizes are enhanced with applied stresses. That effect can be observed in Figure 2.20 (a) by the increase of the voltage in the pick-up coil as the applied stress increases and in Figure 2.20 (b) by a shift towards higher values of the cut-off of the avalanche size power law distribution ${ }^{156}$.
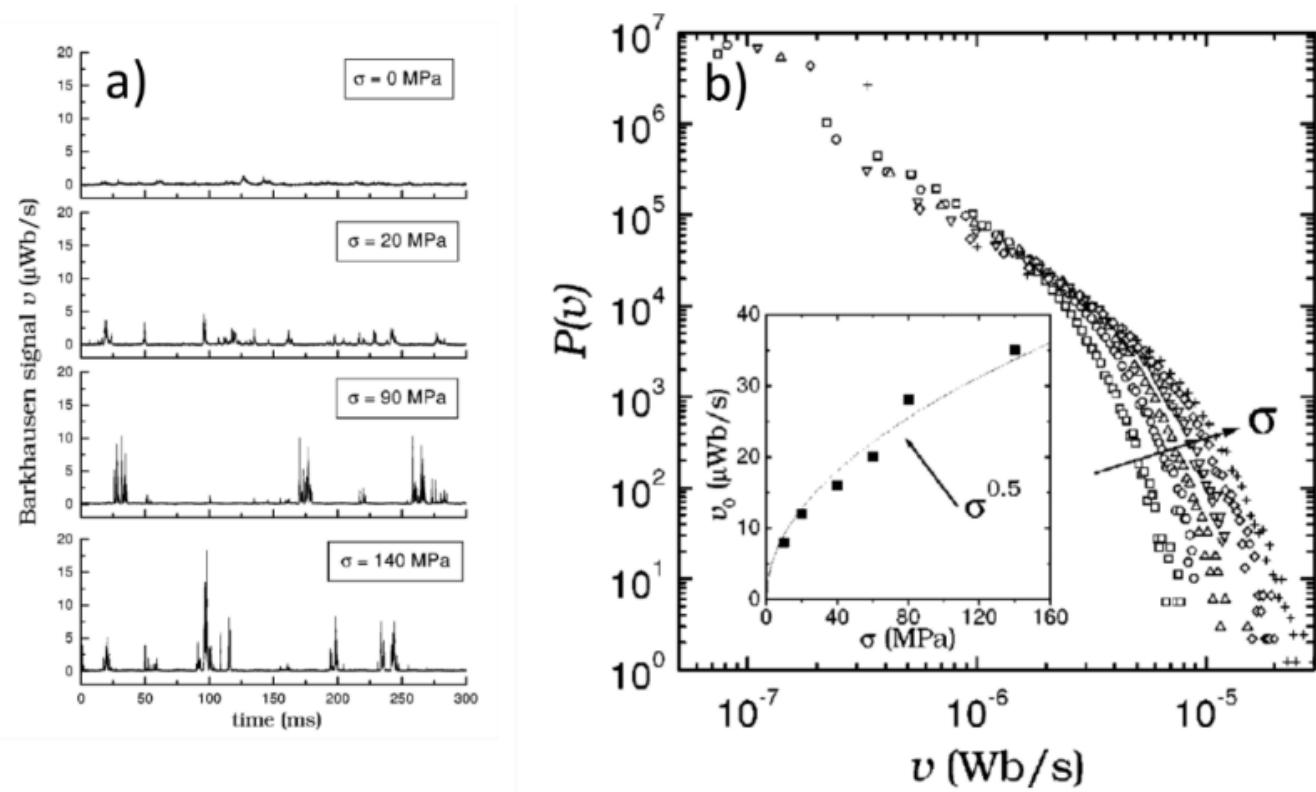

Figure 2.20 (a) Barkhausen noise signal of a magnetic metallic glass for different values of mechanical stress. (b) Probability distribution of Avalanche size (Power of the voltage peaks) for different values of applied stress. The inset shows how cutoff shifts towards higher avalanche sizes as the mechanical stress increases. ${ }^{156}$ 


\section{Experimental techniques}

In this chapter the different experimental techniques used to produce the results discussed throughout this thesis are presented. Each of the subsections introduce briefly the working principle of each of the techniques, as well as the specific features of the used set-up. Additionally, an example is included of an experimental result acquired with the described set-up. Special emphasis was placed in the description of the Dynamic Mechanical Analyzer (DMA) since it was the main tool for the production of the results of this thesis. In particular, it is described in detail the modification that was implemented in such device in order to apply a magnetic field during the mechanical tests.

\subsection{Metallic glass preparation}

The experiments described in this work were performed on several metallic glasses of different compositions. The study of the avalanches in non-magnetic glasses was carried out in Pd-rich ribbons of composition $\mathrm{Pd}_{77.5} \mathrm{Cu}_{6} \mathrm{Si}_{16.5}$, that were prepared by the melt-spinning technique. Two different alloys were used for the experiments on magnetic samples: commercial Fe-rich 2605SA1 alloy with magnetostriction $\lambda_{S}=27 \times 10^{-6}$, provided by Tobias Herold from Metglas Inc., and a Co-rich alloy with nominal composition $\mathrm{Fe}_{2} \mathrm{Co}_{73} \mathrm{Si}_{10} \mathrm{~B}_{15}$ and vanishing magnetostriction, prepared in the Laboratory of Applied Phyisics in laçi by N.Lupu and co-workers. In the following of this subsection, the fabrication details of the $\mathrm{Pd}_{77.5} \mathrm{Cu}_{6} \mathrm{Si}_{16.5}$ samples are summarized.

The first step for the melt-spinning preparation is to gather the right amount of crystalline components to lead to the nominal composition $\mathrm{Pd}_{77.5} \mathrm{Cu}_{6} \mathrm{Si}_{16.5}$ in atomic percentage. To do that, the necessary weight of each of the elements was calculated, so the atomic proportion was kept to $77.5 \% \mathrm{Pd}, 6 \% \mathrm{Cu} 16.5 \% \mathrm{Si}$, for a total weight of approximately $5 \mathrm{~g}$. The required weights for each of the components were estimated making use to the atomic weight of each of the elements and through the following formula: 


$$
P_{i}^{w}=\frac{P_{i}^{a t} W_{i}}{\sum_{j} P_{j}^{a t} W_{j}}
$$

Where $P_{i}^{a t}$ stands for the atomic percentage of the element $(i: \mathrm{Pd}, \mathrm{Cu}, \mathrm{Si}), W_{i}$ represents the atomic weight of each of the elements $\left(W_{P d}=106.4 \mathrm{~g} / \mathrm{mol} W_{C u}=63.55 \mathrm{~g} / \mathrm{mol} W_{S i}=28.09 \mathrm{~g} /\right.$ $m o l)$, and $P_{i}^{w}$ the weight percentage. Substituting the values of $W_{j}$ and $P_{i}^{a t}$ in the equation above, one obtains:

$$
P_{P d}^{W}=90 \% P_{C u}^{W}=4.9 \% P_{S i}^{W}=5.1 \%
$$

which leads for a total amount of $5 \mathrm{~g}$ of sample to $4.5 \mathrm{~g}$ of $P d, 0.245 \mathrm{~g}$ of $\mathrm{Cu}$ and $0.255 \mathrm{~g}$ of $\mathrm{Si}$.

After collecting the right amount of each component, the next step is to prepare a crystalline prealloy. It was done making use of an arc-melter which was previously fluxed and purged six times with $\mathrm{Ar}$ and then kept at a 600 mbar Ar atmosphere. Even in an Ar-rich atmosphere, the pre-alloy might still undergo some oxidation due to the high temperatures achieved during arc-melting. Therefore, as an extra prevention, a Zr sample was also introduced in the arc-melter. Since Zr has a high oxidation tendency, it acts as sinkhole or trap for the oxygen molecules that might still be in the chamber, preventing the oxidation of the actual sample.

Subsequently of the arc-melting, the resulting melt is introduced in a melt-spinner ${ }^{5}$, whose chamber was previously fluxed six times with Ar and afterwards set to an Ar atmosphere of 400 mbar in the chamber and $700 \mathrm{mbar}$ in the pressure tubes. The sample tube's nozzle has $2 \mathrm{~mm}$ of diameter and the Cooper wheel rotation frequency was set to $35 \mathrm{~Hz}$. The sample tube is wrapped by a coil that acts as induction furnace, which reaches a temperature of $1315{ }^{\circ} \mathrm{C}$ before shooting. The shooting of the melt from the sample holder to the wheel is achieved by releasing pressure from the tubes, which presses the melt across the nozzle and fires it against the rotating wheel. When the melt impacts the rotating wheel, it gets quenched in a ribbon shape and gets thrown towards a $4 \mathrm{~m}$ tube which is attached to the extreme of the melt-spinner. This tube provides some flying distance for the quenched melt, which reduces the bents and wrinkles in the final ribbon. 


\subsection{Structural characterization}

The structure of the samples was studied by means of X-ray diffraction (XRD) experiments. XRD working principle is based on the interaction of an incident radiation and the crystallographic planes of a given material: If a crystalline material is illuminated by $\mathrm{X}$-ray radiation, the lattice crystallographic planes will produce destructive and constructive interference of the diffracted wave. The condition for the constructive interference wave is given by Bragg's Law ${ }^{157}$ :

$$
2 d \sin \theta=n \lambda
$$

where $d$ is the interatomic distance of a given family of planes, $\theta$ the angle of diffraction, $n$ is a positive integer and $\lambda$ is the wavelength of the incident radiation.

A crystalline material, which is characterized by structural long range order, produces very sharp peaks on the XRD patterns. Those sharp peaks are a consequence of the evident structural anisotropy of a crystal and the existence of family of planes that act as scattering centers. On the other hand, an amorphous material is characterized by the absence of long range order. This implies that is not possible to define crystallographic planes, and therefore a XRD spectrum of an amorphous sample do not show sharp peaks, but instead it displays the so-called amorphous halo which is related to the Short Range Order (SRO) which is still present in the amorphous state.

In the context of the present work, the main objective with the structural analysis is to make sure that the samples are amorphous. This test is especially important for the self-prepared $\mathrm{Pd}_{77.5} \mathrm{Cu}_{6} \mathrm{Si}_{16.5}$ samples, but it is also crucial for the samples that were mechanically tested at high temperatures, in order to check if the sample crystallized during the test.

Another important aspect that must be taken into account to perform XRD experiments is the wavelength or Energy of the incident radiation. One first point that must be taken care of, is that the energy should be high enough in order to produce radiation with wavelength comparable to the interatomic distance (on the order of Angstroms) to have enough resolution to resolve the structure. A second important aspect is that the incident energy should not match the energy of an electronic transition of an element present in the material under analysis. If that were the case, the incident radiation would be absorbed by such element and it will be later released in the form of fluorescence emission. The consequence of that process would be an extremely low signal to noise ratio in the XRD spectra.

The $\mathrm{Pd}_{77.5} \mathrm{Cu}_{6} \mathrm{Si}_{16.5}$ samples were structurally analyzed making use of a Siemens D8 Diffractometer. Such device is equipped with a $\mathrm{Cu} K_{\alpha}$ radiation source with wavelength $\lambda=$ $1.574 \AA$. The energy of the $\mathrm{Cu} K_{\alpha}$ radiation $(8046 \mathrm{eV})$ is very close to the energy of a K-transition in iron $(7112 \mathrm{eV})$. Therefore, given to the arguments given above, this source is not suitable for the study of the magnetic samples, due to the iron content of both alloys. Consequently, In order to measure the XRD spectra of the magnetic samples a Mo X-ray source was installed in a Siemens D5000 Diffractometer. Such Mo source produces X-ray with $K_{\alpha}=0.71073 \AA$, and allows to measure the X-ray spectra of the magnetic alloys. Figure 3.1 shows two examples of diffraction spectra measured with the Siemenes D8 equiped with a $\mathrm{Cu} K_{\alpha}$ source and the Siemenes D5000 equiped 
with a Mo $K_{\alpha}$ source respectively. None of the spectrum show sharp peaks, which are characteristic of crystalline phase. Instead, an amorphous halo can be appreciated, and in the case of the Fe-rich alloy a second peak can be seen which is related to the medium range order and has been connected with the presence of structures of icosahedral clusters ${ }^{158}$.
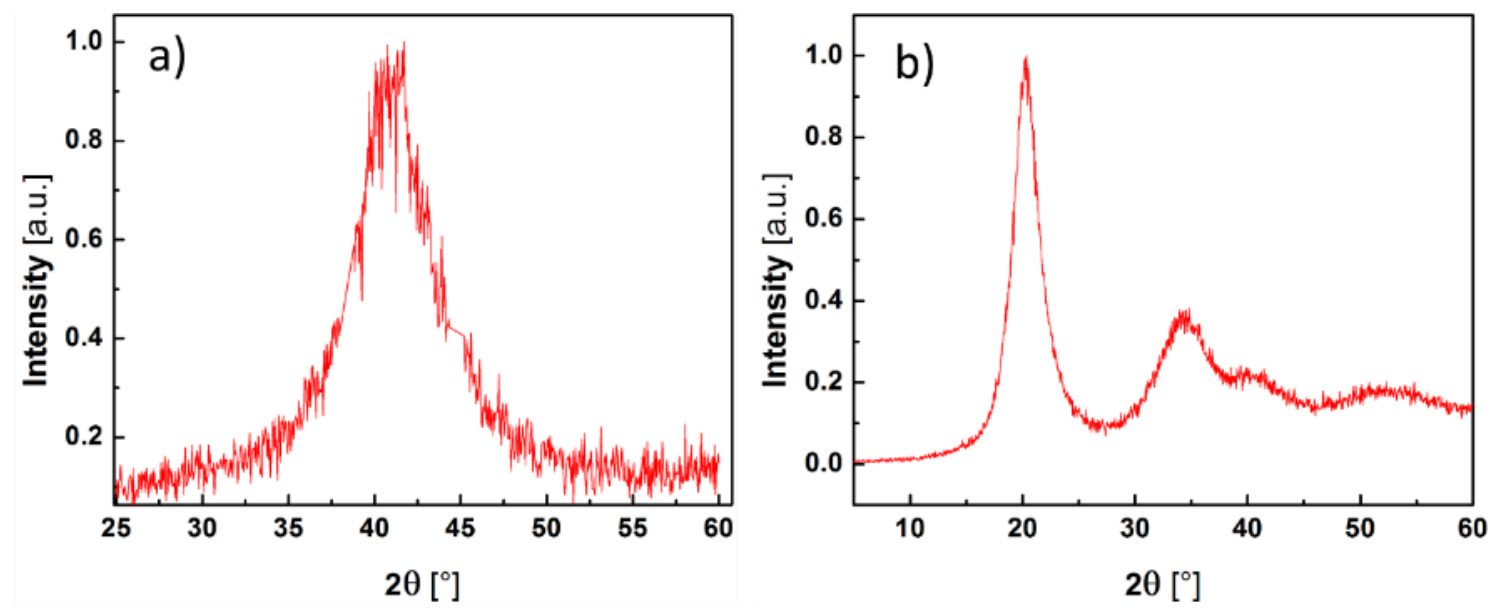

Figure 3.1 (a) XRD spectra of As-cast $\mathrm{Pd}_{77.5} \mathrm{Cu}_{6} \mathrm{Si}_{16.5}$ measured with $\mathrm{Cu} K_{\alpha}$ radiation. (b) XRD spectra of As-cast 2605SA1 measured with a Mo $K_{\alpha}$ source. 


\subsection{Thermal characterization}

The thermal properties of the metallic glasses such as glass transition temperature $\mathrm{T}_{\mathrm{g}}$ or crystallization temperature $\mathrm{T}_{\mathrm{X}}$ were measured by means of a power compensated Differential Scanning Calorimeter DSC-7 from Perkin Elmer. The DSC test measures the amount of heat required to increase the temperature of a sample and a reference (in our case we used an empty pan) as a function of temperature. The actual measured quantity with this technique is the difference between the heat flow between the sample and reference. This heat flow is sensitive to structural rearrangements or relaxation processes, and can be used to experimentally probe $T_{g}$ and $T_{X}$.

Prior to any measurement the DSC must be calibrated. This is done by running two temperature scans on highly pure In and $\mathrm{Zn}$ samples respectively, whose melting temperatures and enthalpy of fusion are known. After performing both runs on the $\mathrm{In}$ and $\mathrm{Zn}$ the experimental values for the melting temperature of $\mathrm{Zn} \mathrm{T}_{\mathrm{Zn}}^{\mathrm{m}}$, melting temperature of $\ln \mathrm{T}_{\mathrm{In}}^{\mathrm{m}}$ and the entalphy of fusion of Indium $\Delta \mathrm{H}_{\text {In }}$, are typed in the calibration wizard so the apparatus corrects it measured values to adjust them to the theoretical ones. It is important to calibrate the DSC using the same temperature rate as the one that will be used for the subsequent measurement, since the calibration parameters are temperature-rate dependent. Once the machine is calibrated, the actual measurement can be started. It consists in a temperature ramp at a certain heating rate $\dot{\mathrm{T}}$ starting from room temperature up to a certain temperature $T_{f}$ such as $T_{f}>T_{X}$, followed by a cooling ramp back to room temperature and finished by a second ramp at the same $\dot{T}$ until $T_{f}$, as is depicted schematically in Figure 3.2 (a). Given that in the moment of the third ramp the sample should be already crystallized, it is used as baseline, that subtracted to the first ramp, leads the typical DSC signal in which $\mathrm{T}_{\mathrm{g}}$ and $\mathrm{T}_{\mathrm{X}}$ can be clearly identified. Figure 3.2 (b) shows a an example of DSC measurement for a $\mathrm{Pd}_{77.5} \mathrm{Cu}_{6} \mathrm{Si}_{16.5}$ ribbon, in which the crystallization temperature $\mathrm{T}_{\mathrm{X}}$ can be measured from the exothermic peak, and the glass transition temperature $\mathrm{T}_{\mathrm{g}}$ can be estimated from the hump prior to the crystallization minimum.

a)

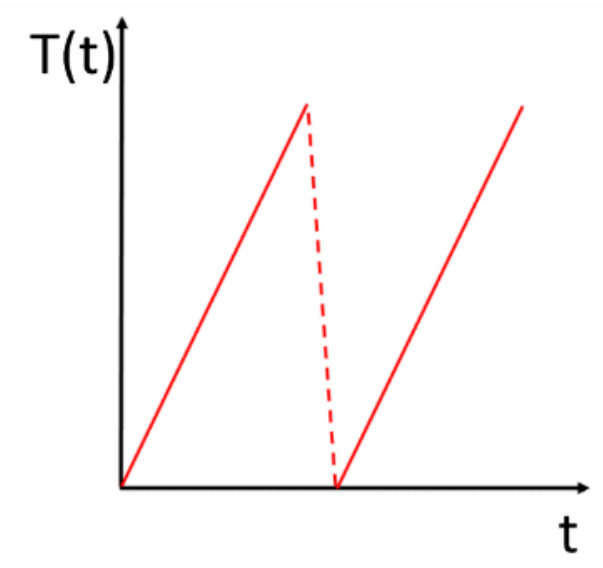

b)

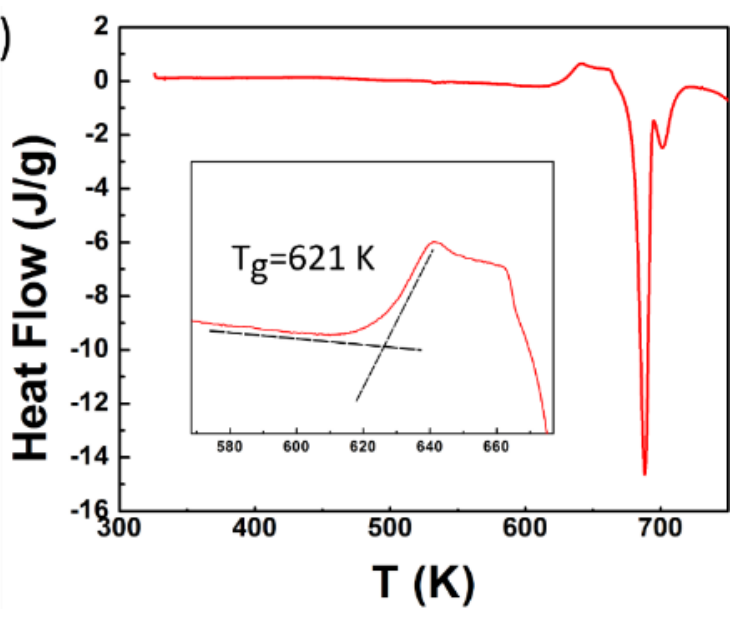

Figure 3.2 (a) Schematic representation of a typical DSC protocol. (b) Example of a DSC measurement carried on an as-cast $\mathrm{Pd}_{77.5} \mathrm{Cu}_{6} \mathrm{Si}_{16.5}$ measured at heating rates of $20 \mathrm{~K} / \mathrm{min}$. $\mathrm{T}_{\mathrm{g}}$ and $\mathrm{T}_{\mathrm{X}}$ can be estimated from the heat flow peak and sharp minimum respectively. 


\subsection{Magnetic characterization}

The $\mathrm{Fe}$ based $2605 \mathrm{SA} 1$ and the $\mathrm{Fe}_{2} \mathrm{Co}_{73} \mathrm{Si}_{10} \mathrm{~B}_{15}$ samples were magnetically characterized measuring their hysteresis loops using a Physical Property Measuring System (PPMS) by Quantum Design set-up with the Vibrating Sample Magnetometer (VSM) option. The VSM operation is based in the Faraday induction law. The sample is oscillated by means of a linear motor near a pick-up coil, and the induced voltage is measured synchronously ${ }^{159}$. A hysteresis loop can be measured by sweeping the DC magnetic field applied to the sample, and measuring the induced voltage, which is proportional to the magnetization of the sample. In addition, the VSM sample holder can be equipped with the oven option, which allows to increase the sample temperature until $500^{\circ} \mathrm{C}$. This option is useful to measure the Curie temperature $T_{C}$ of the magnetic samples. Figure 3.3(a) shows a plane of the main components of the PPMS in the VSM operation mode. Figure 3.4 displays an example of an hysteresis curve and $\mathrm{M}(\mathrm{T})$ curves measured by VSM.

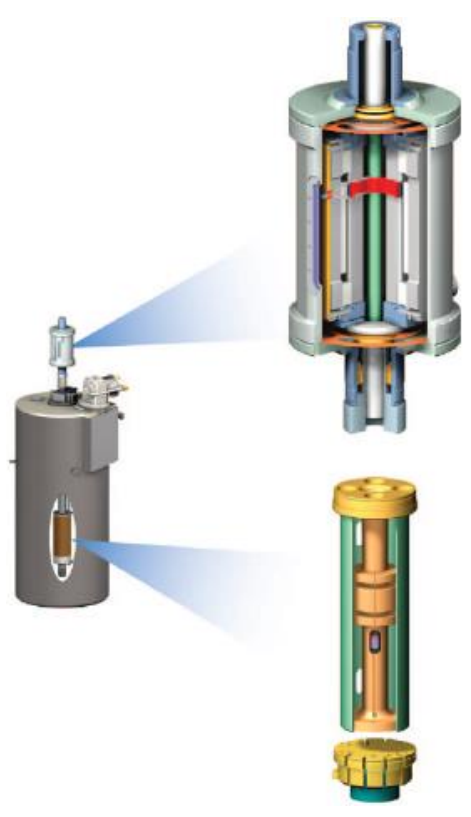

Figure 3.3 Main components of the PPMS equipped with the VSM option from Quantum Design: Linear motor (top) and coil set (bottom) ${ }^{159}$. 

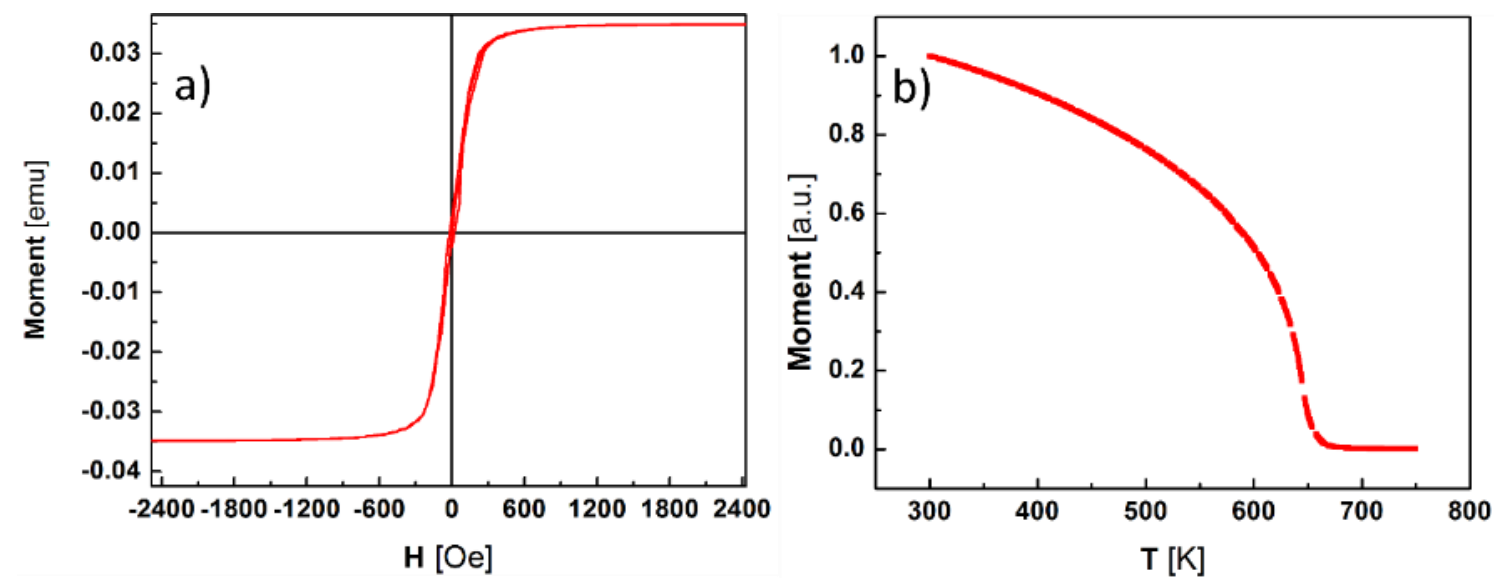

Figure 3.4 (a) Hysteresis loop measured along the width direction of $2605 S A 1$ ribbon. (b) $\mathrm{M}(\mathrm{T})$ vs $\mathrm{T}$ curve of the same ribbon measured with a magnetic field of 300 Oe.

Figure 3.4 (a) shows an example of hysteresis loop. Such measurement allows to measure magnetic properties such as the coercitive field, saturation magnetization, anisotropy field, magnetic remanence and susceptibility. Figure 3.4 (b) displays a Magnetisation vs Temperature curve, which allows to estimate the Curie temperature $\mathrm{T}_{\mathrm{C}}$, as the temperature in which the magnetization vanishes. 


\subsection{Dynamic mechanical analysis (DMA)}

The Dynamic mechanical Analysis (DMA) is a technique to investigate the mechanical properties of materials, typically viscoelastic behavior of polymers and glasses. The historical precursor of the DMA can be found in the first attempts to study elasticity by means of an oscillatory experiments made by Poynting in $1909{ }^{160}$. However, commercial instruments were not available in the market until the 1950's, in which the Weissenberg Rheogoniometer was developed ${ }^{161}$. Although it use was initially constrained to research, DMA has become a standard technique that can be commonly found in analytical laboratories. A DMA allows to stablish a precise Force (either static or dynamic) and temperature on a given sample and to read out accurately the displacement or strain exerted on the sample.

All the mechanical tests in this work were performed making use of a Dynamic Mechanical Analyzer from Perkin Elmer, model DMA-7. A picture of the set-up is shown in Figure 3.5 (a). The analyzer sits on top of an anti-vibration table and is housed in a MBRAUN atmosphere controlled glovebox which is connected to a LEYBOLD TRIVAC B vacuum pump and to a $N_{2}$ source. The purpose of the glovebox is to allow to perform the experiments in a $N_{2}$-rich environment, and avoid oxidation of the samples. The pump is programmed to keep to pressure values between 2.5 and $4.5 \mathrm{mbar}$. The pressure is only reduced when the samples are installed or removed, in order to facilitate the operation inside the glovebox. During the experiments, the pump is disconnected to diminish the vibrations and a glass safety valve is opened instead. This valve is designed to only allow the gas to come out of the glovebox, and allows to release possible overpressure in the chamber during the experiments. The safety valve is shown in the inset of Figure 3.5 (a). The main chamber of the glovebox, where the DMA sits, is accessed through a floodgate. Every sample or tool that needs to be brought to the main chamber, enters through this floodgate. The floodgate is fluxed and pumped 4 times with $N_{2}$ in order to eliminate the oxygen before any item is brought inside the main chamber. Figure 3.5 (b) shows a sketch of the DMA-glovebox set-up. 


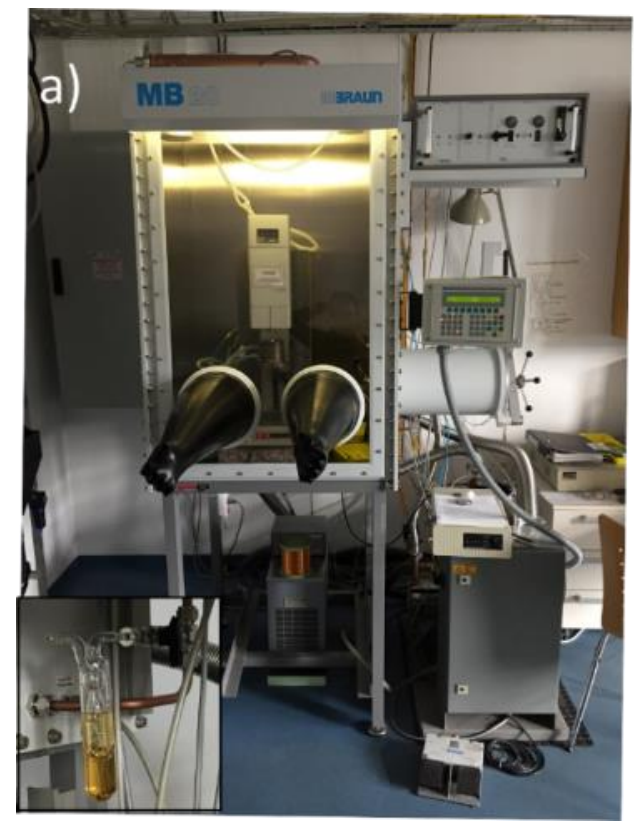

b)

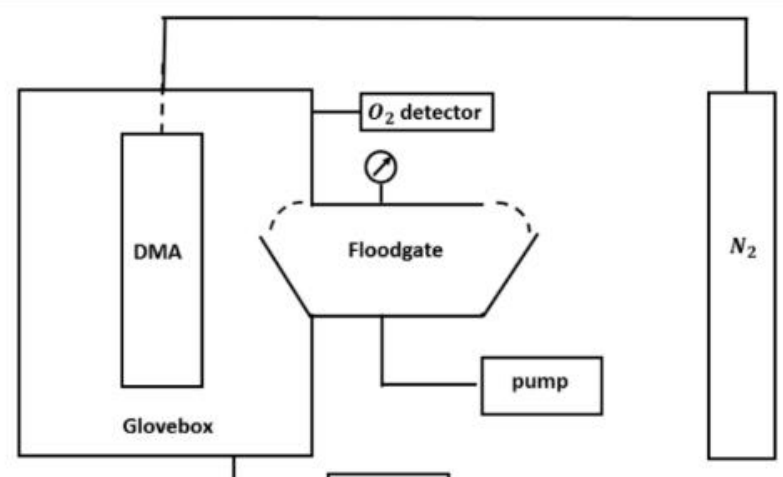

Figure 3.5 (a) Picture of the DMA and glovebox set up in the Mechanical Analysis Laboratory of the $1^{\text {st }}$ Institute of Physics in Göttingen. (b) Sketch of the different components of the glovebox-DMA set-up.

The DMA-7 allows to establish a Force on the range from 0-1000 mN, with a resolution of $0.1 \mathrm{mN}$ and with a frequency in the range $0-100 \mathrm{~Hz}$. The temperature can be increased until $550^{\circ} \mathrm{C}$ with a resolution of $0.1{ }^{\circ} \mathrm{C}$. The main limitation of the measurements is the sample displacement resolution, which is $15 \mathrm{~nm}$ and determines the analysis of the data. The following subsections focus on the description of the DMA operation principle, calibration procedures, and the different experimental protocols used through this work. 


\subsubsection{DMA operation}

The DMA consists in four main components, all of them aligned along the device's vertical axis: The core rod with the measuring system, a displacement detector based in a Linear Variable Differential Transformer (LVDT), a linear motor and a furnace. Figure 3.6 (a) shows an overall scheme of the device.

All the experiments described in this work were performed in tensile geometry on ribbon shaped samples. In such set-up, a quarz probe whose bottom edge is finished in a double-hooked shape is assembled to the bottom of the core rod. Additionally, a hollow cylindrical piece surrounds the quarz probe. Both the quarz probe and the bottom of the hollow cylinder are built with holes that fit in the sample clamps. Previously, the ribbon was attached to a couple of clamps on an aluminum mold that allows to make sure that the system clamp-sample-clamp stays aligned. Both clamps are attached to the hook of the cylinder and to the hollow tube respectively. Once the sample is mounted on the rod, the furnace must be moved upwards so the sample sits surrounded by the furnace during the experiment. A thermocouple is attached to the inner part of the hollow tube in order to measure and control the temperature of the sample. A scheme of the sample housing in tensile device is shown in Figure 3.6 (c).

One of the key components of a DMA is the displacement measurement system, whose accuracy limits the spatial resolution of the apparatus. The DMA-7 uses a Linear Variable Differential Transformer LVDT to measure small displacements. Figure 3.6 (b) provides a schematic view of the LVDT set-up. The LVDT consists in a set of three coils wrapped around a common axis, which in this case is the movable vertical core rod, and it working principle is based on the Faraday induction and Ampere circuital laws. In the initial configuration, the primary coil sits symmetrically around a piece of the rod covered by a magnetically permeable material. The primary coil is feed by an AC current which, following Ampere's law, creates a magnetic flux along the core axis which is enhanced further due to the magnetically permeable piece. That magnetic flux will induce, due to Faraday's Induction law, an electromotive force on both secondary coils giving rise to measurable voltages $V_{1}$ and $V_{2}$ in both coils respectively. In the initial configuration, in which the primary coil sits exactly centered on the magnetic piece, the electromotive force induced in both coils is, for symmetry reasons, exactly the same. However, if the central rod moves, and therefore the magnetic piece, such symmetry will be broken and the induced voltage on both secondary coils will be different. Knowing the characteristics of the coils and magnet, the displacement of the rod can be backtracked from the voltage difference in both coils. Finally, since the sample is attached to the central rod, any displacement of the rod is associated to sample deformation.

Lastly, the DMA makes use of a linear motor to apply the force, and a ceramic furnace to bring the sample into the desired temperature. The oven is set around the sample at the bottom of the DMA structure, and it is surrounded by a cooling block with a fluid flow in order to keep the heat flow constrained in the sample's housing area. 


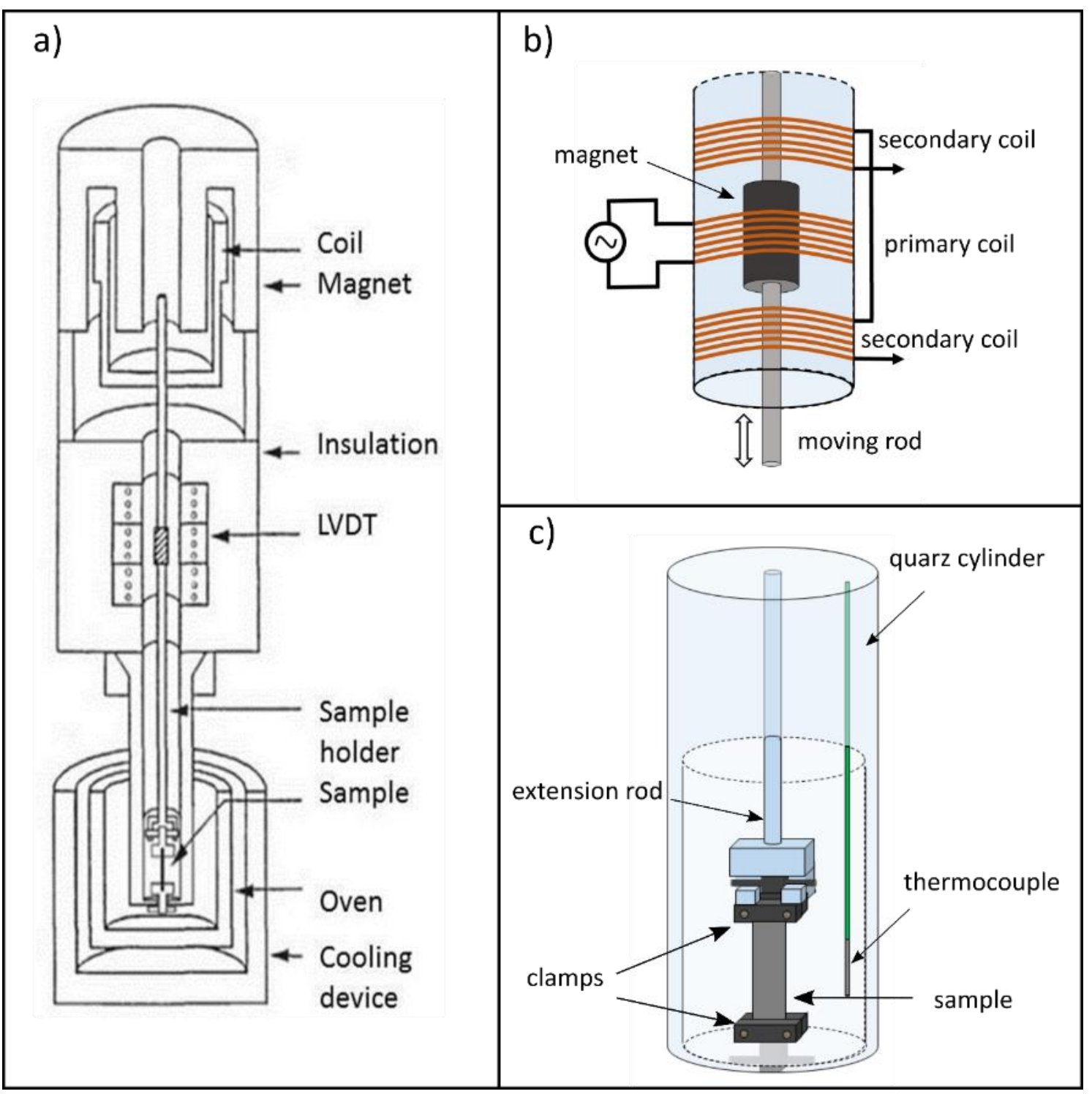

Figure 3.6 (a) Cross-section of the Dynamic Mechanical Analyzer and it main components. (b) Sketch of the LVDT. (c) Illustration of the DMA tensile set-up. 


\subsubsection{DMA calibration}

In order to assure the accuracy of the measurements is it of foremost importance that the DMA is properly calibrated. A full calibration is recommended to be done once a year and every time that any component is changed. The calibration is done through a calibration wizard that guides the user through several steps that must be done in order. The whole DMA calibration procedure is summarized in the following paragraph, a more detailed version of the calibration protocol can be found in the official user manual ${ }^{162}$ :

1. Internal friction calibration: This must be done before starting the calibration wizard. It measures the friction of the inner core, which may increase due to a misalignment of the machine. It must be kept to values lower than $80 \mu \mathrm{N}$. If that's not the case the supporting legs of the DMA must be adjusted until the friction value is reduced.

2. Restore defaults: The calibration parameters must be set to the default ones before starting the calibration wizard. Before starting, the DMA must be mounted with the parallel Plate kit which is required for the first calibration steps.

3. DMA calibration: This is the name of the first step in the calibration wizard. In this step the system calibrates the friction as a function of the frequency, the amplitude of the sine wave, system damping and inertia.

4. Height Calibration: This step is used to calculate the ordinate axis of the DMA. It is done using a $10 \mathrm{~mm}$ height displacement standard. The system measures the ordinate value and the value is corrected to fit the known value of the standard.

5. Force calibration: In this step a $50 \mathrm{~g}$ standard must be placed on a plastic tray on top of the central rod. In an analogous way as for the height standard, the software measures the force exerted by the standard and corrects the measured value with the expected one.

6. Eigendeformation calibration: This is the procedure to estimate the system compliance. To do that, a steel cylinder calibration standard is used, the DMA presses against it and the displacement values are recorded. After doing this calibration, the compliance of the system is subtracted from every measurement.

7. Temperature calibration: This is the procedure for calibration of the thermocouple used to measure the temperature. The thermocouple is attached directly to the outer cylinder, which is a different model depending in the experimental set-up. Therefore, before performing this calibration the set-up must be changed to the definitive one that will be used in our experiments (tensile-set up). This way, the temperature calibration will be performed with the thermocouple in the exact same position as it will be during the experiments, increasing the accuracy of the calibration. In this procedure, a temperature scan is run over two standard samples of $\mathrm{Sn}$ and $\mathrm{Zn}$ whose melting points are known $\left(\mathrm{T}_{\mathrm{m}}^{\mathrm{Sn}}=\right.$ $231.9^{\circ} \mathrm{C}, \mathrm{T}_{\mathrm{m}}^{\mathrm{Zn}}=420^{\circ} \mathrm{C}$ ). The temperature scans are done applying the minimum force required to keep the samples straight in the holder. From the experimental elongation vs temperature data, the temperature at which the sample breaks $\left(\mathrm{T}_{\mathrm{m}, \exp }^{\mathrm{Sn}}, \mathrm{T}_{\mathrm{m}, \exp }^{\mathrm{Zn}}\right)$ can be estimated. Comparing those values with the theoretical ones, the software performs a regression and calibrates the temperature.

8. Furnace calibration: This last step performs a calibration between the user chosen upper and lower limit temperatures for the oven operation. This is an automatized process and after it the DMA is ready to be used. 


\subsubsection{Creep measurements}

A creep test consists in the measurement of the deformation as a function of time under the application of a constant stress at constant temperature ${ }^{163}$. Given the simplicity of the protocol, it is one of the most fundamental mechanical tests that can be made. Despite of the simplicity of the test, creep flow is broadly used to characterize materials and analyze their viability for certain applications, i.e., big amount of creep deformation would be catastrophic in turbine blades, since that may lead to contact their housing and the failure of the piece, whereas some creep in concrete can be positive since it relieves tensile stress that may otherwise lead to cracking.

Typically, a creep test is preceded by some temperature equilibration, in which the sample is kept for some time at the working temperature but with a small applied force. The small force is needed when working in tensile geometry to keep the sample straight during the equilibration. Also very often, after the creep measurement the force is reduced again to a small value and the deformation evolution is analyzed. Such behavior is called "recovery", and when it is also studied the whole experiment is referred as "creep-recovery" test. A schematic illustration of a creep-recovery protocol is shown in Figure 3.7 (a). Classic examples of information that can be retrieved from a creep-recovery test are the elastic, anelastic and plastic strain $\varepsilon_{e l}, \varepsilon_{a n}, \varepsilon_{p l}$ and the shear viscosity $\eta$, that can be calculated from the regime of quasi-steady state plastic flow through the equation: $\eta=\frac{\sigma}{3 \dot{\varepsilon}} 164$. Figure 3.7 (b) shows a schematic creep curve in which the magnitudes $\varepsilon_{e l}, \varepsilon_{a n}, \varepsilon_{p l}$ are indicated.

a)

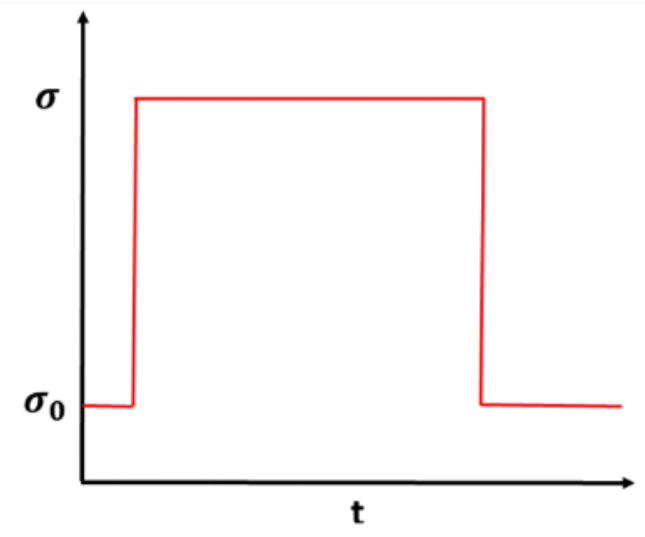

b)

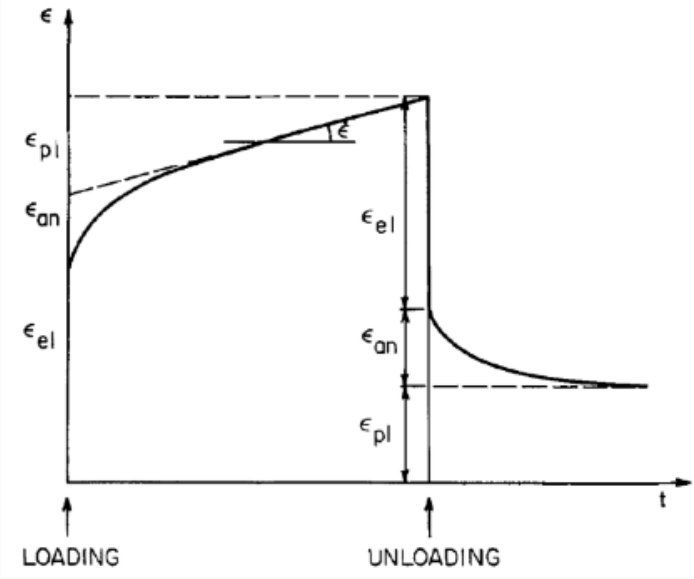

Figure 3.7 (a) Schematic representation of a creep-recovery protocol. (b) Schematic creep curve, reproduced from ${ }^{163}$. 


\subsubsection{Stress-strain measurements}

This experimental protocol consists on the application of a stress ramp to a sample kept at a constant temperature. In this thesis, all the stress-strain curves were done keeping a constant stress rate $\dot{\sigma}$. Although it can also be done adjusting the stress rate to keep a constant strain rate $\dot{\varepsilon}$, we chose the former protocol for simplicity. The reason is that the DMA analysis is intrinsically stresscontrolled. Therefore, the attempt to measure with a constant strain rate condition implies the use of an internal feedback loop that hampers the analysis of the fine structure of the data. The differences between the stress-controlled and strain controlled protocols, in the context of the avalanche analysis will be discussed in detail in following sections of the thesis.

Commonly, the result of a stress-strain experiment is represented by plotting the applied stress in the abscissa axis and the strain in the ordinates, leading to the so-called stress-strain curve. If the applied stress and/or temperature are high enough, two regimes can be distinguished in a stressstrain curve. The first one is the elastic regime, and it is characterized by a linear relationship between stress and strain following Hooke's law. Although the actual existence of a purely elastic regime in metallic glasses is currently questioned, as was discussed in the previous chapter ${ }^{152}$, the elastic regime was historically defined by a good fit of the Hook's law to the stress-strain curve, regardless of the fine structure of the data. The Elastic Modulus (Young modulus in the case of tensile uniaxial deformation) can be determined from the elastic region as the proportionality constant between the stress and strain: $\sigma=E \varepsilon$. If the stress overcomes a certain value called yield stress $\sigma_{Y}$, the sample undergoes the plastic regime, which is identified by a constant average stress value. The ductility of a sample can also be characterized by the amount of strain that the sample undergo after reaching the yield stress and before failing. Although metallic glasses generally show almost no tensile ductility at room temperature, which leads to brittle fracture almost immediately after reaching the yield stress, many attempts have been made to increase the metallic glass ductility, as was discussed in the previous chapter. Figure 3.8 displays a sketch of a stress-controlled stress-strain protocol and an example of two stress-strain curves of metallic glasses in tension and compression.
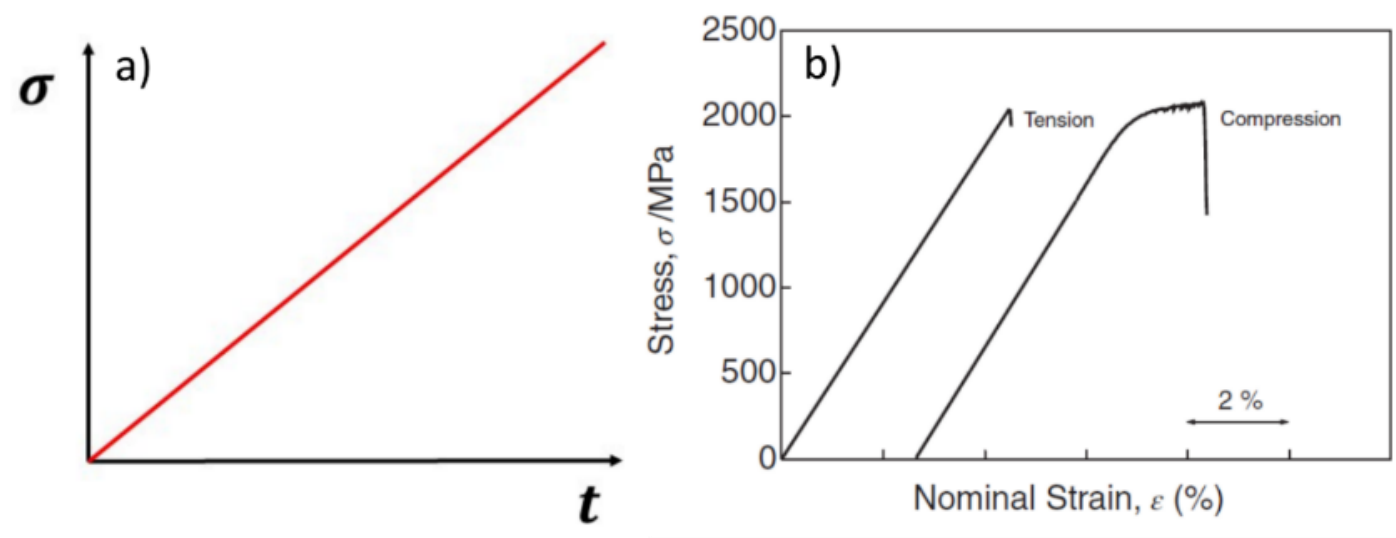

Figure 3.8 (a) Sketch of a stress-controlled stress-strain protocol. (b) Experimental stress-strain curves of Ti-based Bulk metallic glasses measured in tension and compression respectively ${ }^{165}$ 


\subsection{Magnetic-DMA analysis}

One of the main objectives of this thesis is the study of the deformation behavior of metallic glasses under simultaneous magnetic and mechanical load. Therefore the DMA had to be modified to be able to apply a magnetic field while conducting the standard mechanical tests. Such magnetic field is generated by several arrangements of permanent magnets surrounding the sample holder. Those permanent magnets were installed in tailored aluminum magnet holders, which kept them fixed and at a controlled position. This way, standard mechanical tests (creep-recovery, stress-strain and others) could be performed with the superposition of a magnetic field $\overrightarrow{\mathrm{H}}$ to the mechanical field $\vec{\sigma}$. In the following paragraph, the two holders that were used are described in detail, as well as the permanent magnets and their calibration. To conclude, an overview of the available range of intensities and orientations with our set-up is given, and a test of the lack of interferences of the magnetic fields with the magnetically sensitive components of the DMA is provided.

When operated in tensile geometry, a ribbon shaped sample is placed vertically inside the cylindrical oven, surrounded by an also cylindrical cooling block, in a way in which the applied stress is parallel to the length of the ribbon. The magnet holders are placed in the space between the furnace and the cooling block, as is depicted in Figure 3.9 (a).

Two different aluminum holders were built and designed to be able to establish magnetic fields along different orientations. The first holder consists in a hollow cylindrical tube that fits between the furnace and the cooling, leaving a small space in between to diminish thermal conductivity. This cylindrical shape is perforated by two opposite rectangular cavities of $36 \times 14 \times 7 \mathrm{~mm}$ that allow to place a pair of rectangular permanent magnets, situated one in front of the other. The height $h$ and distance $d$ between the magnets can be adjusted placing Aluminum spacers between the magnets and the base and inner diameter of the holder respectively. Figure 3.9 (b) shows a picture of this magnet holder, and the spacers. Such set-up was used in combination with rectangular permanent magnets built with the magnetic poles in the opposite faces. This way, a nearly constant magnetic field can be stablished along the plane of the section of the sample, in a crossed field geometry respect to the stress. The intensity of the magnetic field can be adjusted changing the spacing $d$ and the magnet material. NdFeB and Sm2Co17 permanent magnets of different thicknesses were used for this purpose. The magnetic field produced by each of the magnet-pairs as a function of the spacing distance $d$ was calibrated with a Hall probe outside of the glovebox. In order to maximize the accuracy of the calibration, we reproduced the DMA environment by placing a furnace and cooling block in the calibration set-up. Figure 3.10 shows the calibration curves for the three rectangular permanent magnets used in the first holder. It can be seen in Figure 3.10, that combining the different materials and spacing $d$, the set-up allows to establish a magnetic field $\vec{H}_{1}$ in the range 100-1000 Oe. Finally, by rotating the holder, the angle $\theta$ between the magnetic field and the ribbon width direction can be accurately controlled.

The second device was designed to house two cylindrical shaped permanent magnets in circular holders. The circular holders are $20 \mathrm{~mm}$ diameter pieces perforated with rectangular cavities of $6 \mathrm{x}$ $6 \times 12 \mathrm{~mm}$. Figure 3.9 (c) shows a schematic representation of the holder. This set-up allows to rotate the magnets within the plane of the applied stress. In combination with magnets polarized along the axial direction, it allows to establish a magnetic field $\vec{H}_{2}$ and to control the angle formed with the stress field $\phi$. In this case there is no room to change the spacing of the magnets, and the 
intensity must be kept constant. This holder was feed by a pair of cylindrical axially polarized AlNiCo magnets, which produce a magnetic field of 120 Oe on the sample position. An overview of the experiment geometry and the different angles that can be accessed with both magnet holders is shown in Figure 3.9 (d). Last, tailored clamps made of non-magnetic steel were used in order to avoid artifacts coming from the interaction of the magnetic field with the clamps.

a)

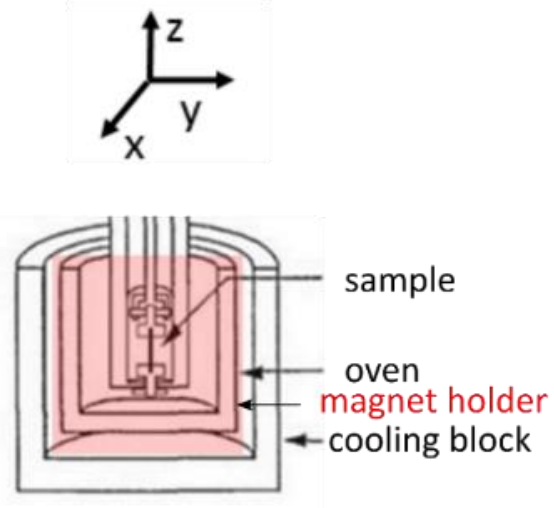

c)

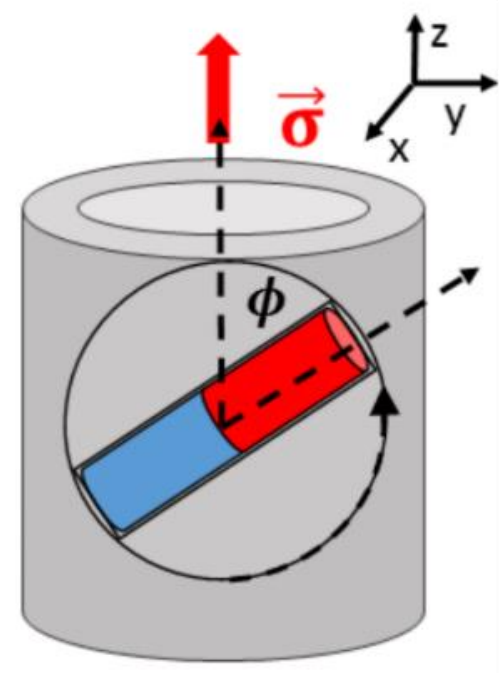

b)

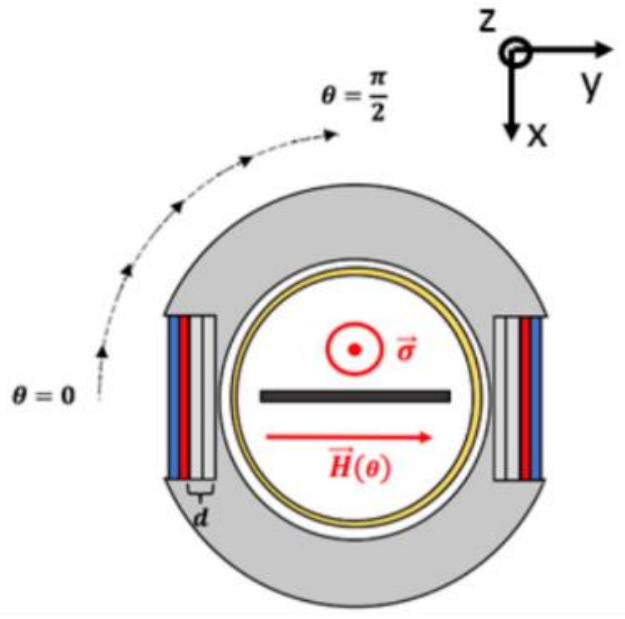

d)

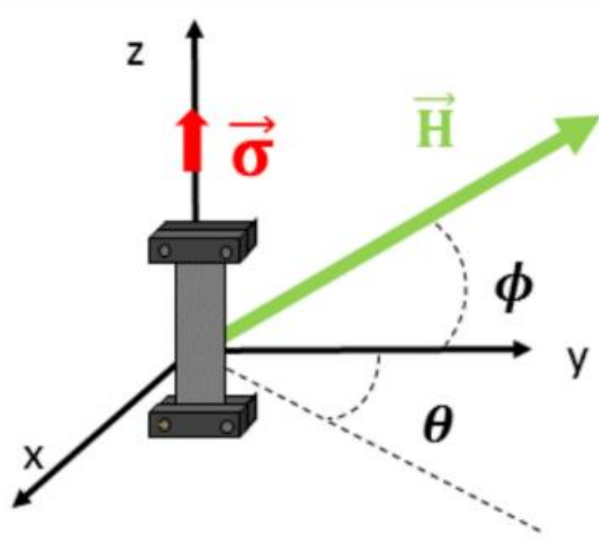

Figure 3.9 Magnetic Dynamic Mechanical Analyzer

(a) Location of the magnet holder in the DMA. (b) Top view of the magnet holder designed to host rectangular permanent magnets. (c) Schematic front view of the magnet holder designed to host cylindrical magnets. (d) Overview of the magneticDMA geometry and the different orientations that can be accessed with both magnet holders.

An Important consideration that must be taken into account is that operating the Magnetic-DMA set-up limits the working temperature of the DMA due to the Curie temperature of the magnets. Table 3.1 displays the maximum operation temperatures of the magnets used in this work. It can be appreciated, that the material choice involves to find a compromise between the maximum operating temperature (limited by the $\mathrm{T}_{\mathrm{c}}$ of the material) and intensity of the produced field. In this 
context, it is worth to be noticed, that due to their low $T_{c}, N d$ magnets were not suitable for creep experiments, which require high temperatures.

Table 3.1 Maximum operation temperatures of the different magnets

\begin{tabular}{|c|c|}
\hline Magnet type & Maximum operation Temperature \\
\hline $\mathrm{NdFeB}(25 \times 10 \times 4 \mathrm{~mm})$ & $80^{\circ} \mathrm{C}$ \\
\hline $\mathrm{Sm}_{2} \mathrm{Co}_{17}(25 \times 10 \times 3 \mathrm{~mm})$ & $300^{\circ} \mathrm{C}$ \\
\hline $\left.\mathrm{Sm}_{2} \mathrm{Co}_{17}(20 \times 6 \times 1.5 \mathrm{~mm})\right)$ & $300^{\circ} \mathrm{C}$ \\
\hline $\mathrm{AlNiCo}(5$ dia $\times 10$ thick$) \mathrm{mm}$ & $500^{\circ} \mathrm{C}$ \\
\hline
\end{tabular}

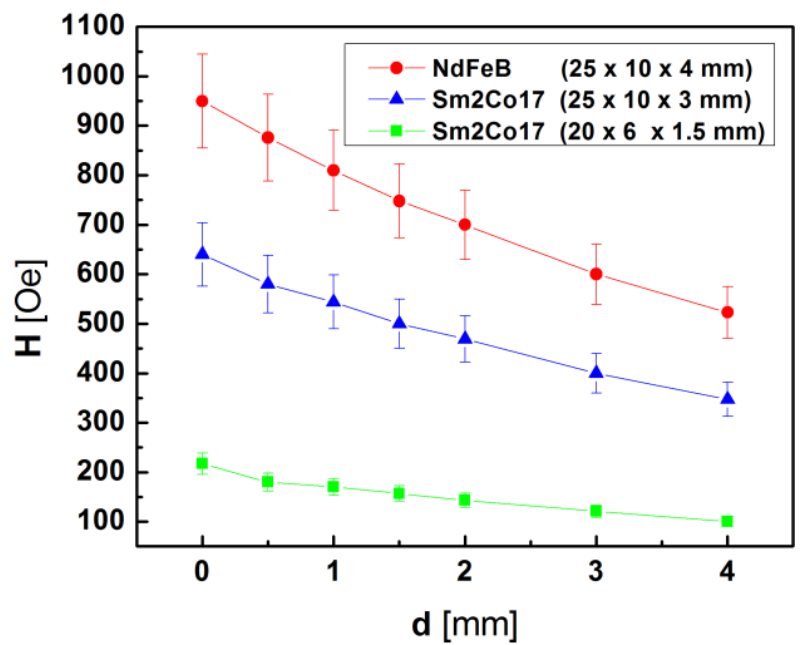

Figure 3.10 Calibration of permanent magnets: $\mathrm{H}$ vs $d$ curves for the three rectangular magnets measured with a Hall probe at room temperature.

Prior to the measurements, it is important to make sure that the magnetic field generated by the magnets is confined in the sample holder area and do not affect the measurement device itself, like the LVDT or linear motor. In this sense the shape of this particular DMA model (DMA-7) is an advantage because the LVDT is placed approximately $20 \mathrm{~cm}$ above the sample holder. That test was done performing several mechanical measurements with the non-magnetic $\mathrm{Pd}_{77.5} \mathrm{Cu}_{6} \mathrm{Si}_{16.5}$ samples, with and without the highest achievable magnetic field (950 Oe).Figure 3.11 shows the result of a stress-strain curve of $\mathrm{Pd}_{77.5} \mathrm{Cu}_{6} \mathrm{Si}_{16.5}$ with and without magnetic field. It can be seen that there are no significant differences in the outcome, which demonstrates that the measuring device itself is not affected by the field. 


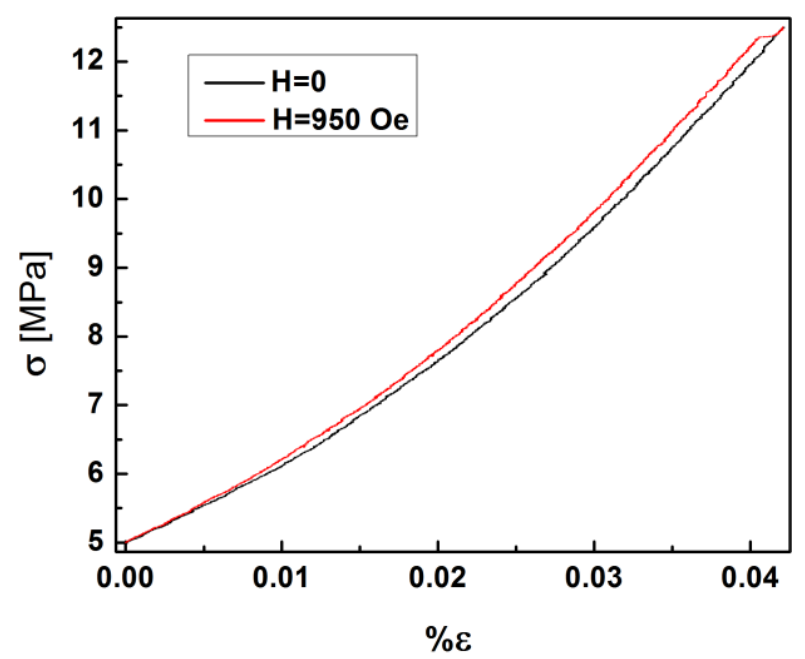

Figure 3.11 Experimental stress-strain curves of $\mathrm{Pd}_{77.5} \mathrm{Cu}_{6} \mathrm{Si}_{16.5}$ samples measured at room temperature at zero field (black line) and at $\mathrm{H}=950$ Oe (red line) 


\section{$4 \quad$ Analytical methods}

In the previous section the experiments which provided the data that have been studied in this work were introduced. This chapter focuses on the description of the analysis procedures of such experimental data. In particular, the filtering procedure, the statistical analysis, and the different figures of merit that have been used to interpret the data are be discussed. Particular attention is payed to take into account the finite spatial and temporal resolution of the experiments. These limitations, that are intrinsic to any experimental measurement, play a basic role in the choice of the figures of merit. This chapter is divided in two subsections, which focus on the analysis of the data produced by creep and stress-strain measurements respectively. 


\subsection{Analysis of creep curves}

\subsubsection{The waiting time $(\Delta t)$ analysis}

As previously defined in Chapter 3, a creep curve displays the evolution of the strain as a function of time under the application of a constant stress $\sigma$ and at a given temperature T. This thesis is concerned with the study of tensile creep on ribbon shaped samples, in which the deformation takes place mainly along the ribbon length axis.

Figure 4.1 (a) shows a creep curve of a $\mathrm{Pd}_{77.5} \mathrm{Cu}_{6} \mathrm{Si}_{16.5}$ sample measured at a constant tensile stress $\sigma=25 \mathrm{MPa}$ and temperature $\frac{\mathrm{T}}{\mathrm{T}_{\mathrm{g}}}=0.95$ during two hours. A first glance of the data shown in Figure 4.1 (a) might suggest that the deformation takes place smoothly. However, a closer look reveals a non-periodic, jerky fine structure of horizontal steps all of them separated by a distance $\Delta \mathrm{L}=15$ $\mathrm{nm}$, which is the DMA spatial resolution. Figure 4.1 (b) displays a zoom of the experimental data in the period of time between 16790 and 16850 seconds, where a structure of horizontal arrays of data points separated $15 \mathrm{~nm}$ can be seen.
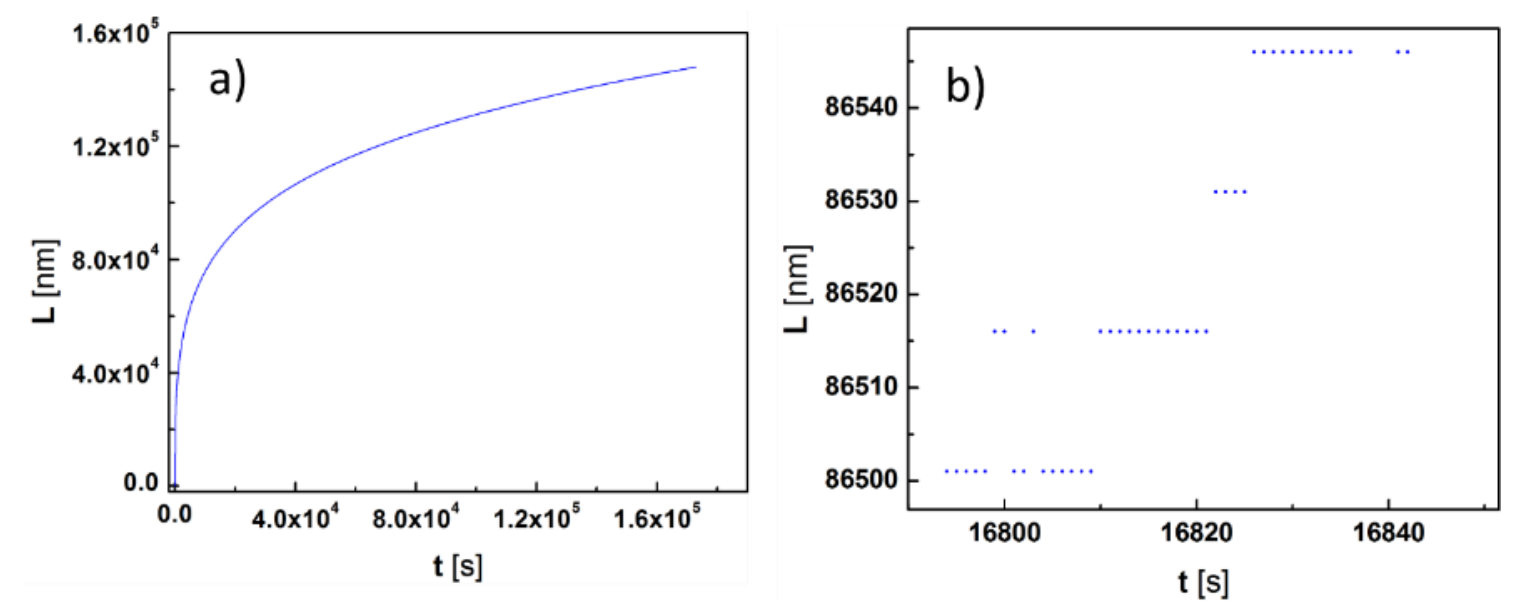

Figure 4.1 (a) Tensile creep deformation of a $\mathrm{Pd}_{77.5} \mathrm{Cu}_{6} \mathrm{Si}_{16.5}$ ribbon subjected to a stress of $\sigma=25 \mathrm{MPa}$ and temperature $\frac{\mathrm{T}}{\mathrm{T}_{\mathrm{g}}}=0.95$ during two hours. (b) Zoom of the creep deformation in the interval $t=(16790-16850)$ seconds, which reveals a staircase -like fine structure of horizontal jumps separated by $15 \mathrm{~nm}$.

After a process of filtering which is detailed in the following paragraph, the horizontal steps that can be appreciated in Figure 4.1 (b) will be referred as waiting times $\Delta t^{\prime}$ 's. These will be the main quantities for the analysis of the creep measurements. In some parts of the analysis the notation $\Delta t(t)$ will be used. In that case $\Delta t$ refers to the waiting time, (the length of the horizontal step) and $t$ indicates the experimental time in which that waiting time starts, i.e. the position of the first point of the horizontal step.

It should be noticed that the staircase-like shape of the fine structure of the data is a consequence of the limits in the spatial resolution of the apparatus. This constrain makes impossible to resolve individual deformation events, which- as discussed in the theory section- are expected to take place 
in length scales below this resolution limit. Thus, a waiting time $\Delta t$ represents the required time to accumulate enough deformation events so the total deformation exerted by the sum of them adds up to $15 \mathrm{~nm}$. The non-resolvable microscopic deformation activity could be classified into a succession of avalanches of different magnitudes $\Delta L_{A V}$ and durations $\Delta t_{A V}$, and some periods of absence of activity or inter-event times $\Delta t_{i t}$. Taking that into account, a waiting time $\Delta t$ can be defined as the sum of the avalanche durations and inter-event times that take place until the total deformation carried out by the avalanches reach a value of $15 \mathrm{~nm}$. This condition can be mathematically expressed as:

$$
\Delta t=\sum_{i} \Delta t_{A V_{i}}+\sum_{j} \Delta t_{i t_{j}} \quad \text { for } \sum_{i} \Delta L_{A V_{i}}=15 \mathrm{~nm}
$$

Since none of the quantities $\Delta t_{A V}, \Delta t_{i t}$ nor their number $i, j$ can be experimentally resolved, there are infinite possibilities of deformation patterns that may lead to a given waiting time.

Figure 4.2 (a) presents a sketch of a waiting time (blue line) and two possible non-resolvable trajectories that lead to the same experimental waiting time (red lines). It illustrates the fact that different microscopic deformation processes may produce the same waiting time. Figure 4.2 (b) depicts the different components of the fine structure of a waiting time, the avalanches (with duration $\Delta t_{A V}$ ) and the inter-event times $\Delta t_{i t}$.

a)

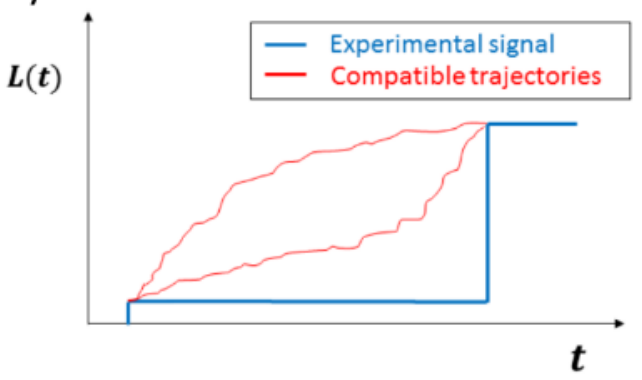

b)

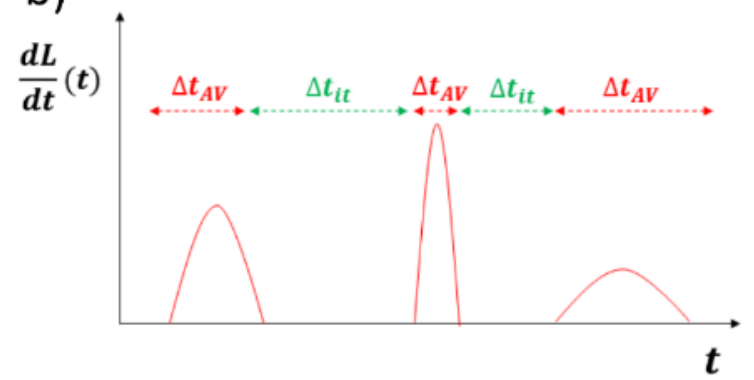

Figure 4.2 (a) Plot of a waiting time(blue line) and two deformation trajectories (red lines) that would give rise to that waiting time (L: length, t: time). (b) Representation of the main components of an inttermittent deformation process: i) Avalanches: characterized by their size, duration and shape and ii) inter-event times. The avalanches have been plotted with a symetrical shape for simplicity.

In conclusion, the variable waiting time $\Delta t$ provides a coarse-grained quantity which is inversely proportional to the avalanche activity. i.e. short waiting times are related with periods of higher deformation activity and vice versa. However, given the already mentioned experimental limits in spatial resolution, it is not possible to know the nature and distribution of events that lead to a given waiting time. 


\subsubsection{Data filtering}

As stated in the paragraph above, raw experimental data should be filtered in order to translate the fine structure of the creep curve to a set of waiting times. The reason is that the creep curve is affected by instrumental noise, which results in the observed down jumps in the fine structure of the data. Such jumps, which would imply a shortening of the sample, are unphysical under applied tensile stress and have to be filtered out.

In a previous work, J.O. Kriponeit et al., proposed a filtering method to determine the transition between two adjacent horizontal levels, and to define the waiting time between them ${ }^{30}$. Their method is based in a comparison of the percentages of data points of two adjacent levels before and after each step position, in order to define one of each data points as the transition between two waiting times. The step position assigned as transition position is the first one that, leaves less data points remaining from the upcoming step than data points have been passed from the preceding step. In both cases such values are normalized with respect to the number of data points in each of the steps. In order to compute this position, one defines two variables $P(t), q(t)$ which refer to two adjacent levels, as follows

$P(t)$ defines the normalized number of points counted in the lower level at the temporal step $t$ and is defined by:

$$
P(t)=\frac{\sum_{t^{\prime}<t} p(t)}{N_{d}}
$$

Where $\sum_{t^{\prime}<t} p(t)$ represents the total number of points counted until the time position $t$ in the lower level, and $N_{d}$ is the total number of points in the lower level.

$q(t)$ is the normalized number of points at the upper level that still have not been counted $t$ a given temporal step $t$, and can be defined by:

$$
q(t)=\frac{\sum_{t^{\prime}>t} p(t)}{N_{u}}
$$

Where $N_{u}$ denotes the total number of points in the upper level.

Making use of the functions $P(t)$ and $q(t)$, the transition between two adjacent levels is determined by the minimum time $t$ which fulfills

$$
P(t)>q(t)
$$

The experimental data is filtered by applying this technique to every pair of adjacent levels, so the final result is a staircase-like shape, which corresponds to a sequence of waiting times $\Delta t(t)$. Figure 
4.3 displays a schematic illustration of the filtering method, where the transition time has been chosen by comparing the functions $P(t), q(t)$.

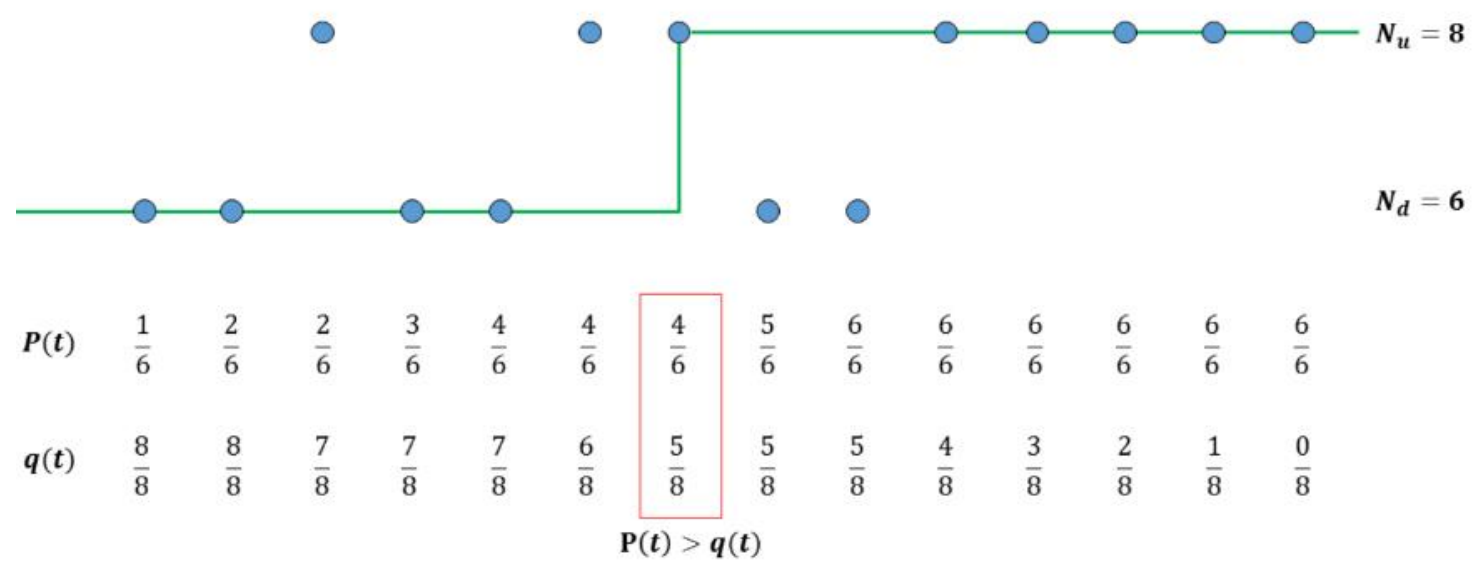

Figure 4.3 Illustration of the filtering method. The corresponding algorithm compares the value of the functions $P(t), q(t)$ between each pair of horizontal steps. The values of $P(t), q(t)$ are shown for each time step, and the transition or jump is defined by the shortest time that fulfils $P(t)>q(t)$.

\subsubsection{Data binning and logarithmic binning}

In the context of avalanche dynamics, data interpretation is based in the analysis of distributions of certain quantities. Those distributions are estimated by histograms of the variables under study, and such process implies that data must be binned. Binning consists on dividing the entire range of values into a series of intervals and then count how many values fall into each interval. The histogram is then drawn by plotting the center of the bins in the $x$-axis and representing the number of cases that fell on each of intervals in the respective $y$-axis. This process implies a certain degree of arbitrariness, since the number of bins has to be decided by the analyst.

A particular case of binning which is especially relevant for the analysis presented in this work is the so-called logarithmic binning. This method is suitable for data sets whose histograms will be represented in double-logarithmic scale. It is particularly recommended for statistical ensembles in which the probability of certain value $P(x)$ decreases dramatically with the magnitude of the value $x$. Logarithmic binning is defined in a way that produces an equidistant set of bins in the logarithmic scale. That is achieved by defining bins with an appropriate increasing size in the original linear space. Since in this case, bins have different widths, the total number of counts in each bin should be normalized by the bin width, which provides an estimate of the probability density.

Figure 4.4 below, shows the difference between linear and logarithmic binning. This is done by comparison of the histograms calculated with each method for the same waiting time set. Figure 4.4 (a) displays the representation in linear space of the probability distribution of waiting times, computed by linear (red) and logarithmic (blue) binning for the same number of bins. Notice that the distance between the blue dots, which correspond to the bin centers, increases with the waiting time size. Figure 4.4 (b) shows the same distributions represented in logarithmic scale. In this case the blue curve, which corresponds to logarithmic binning extends to a wider range, and bin centers turn out to be equidistant. This is in sharp contrast with the distribution calculated with linear binning, which shows an accumulation of bin centers at the right of the histogram. 

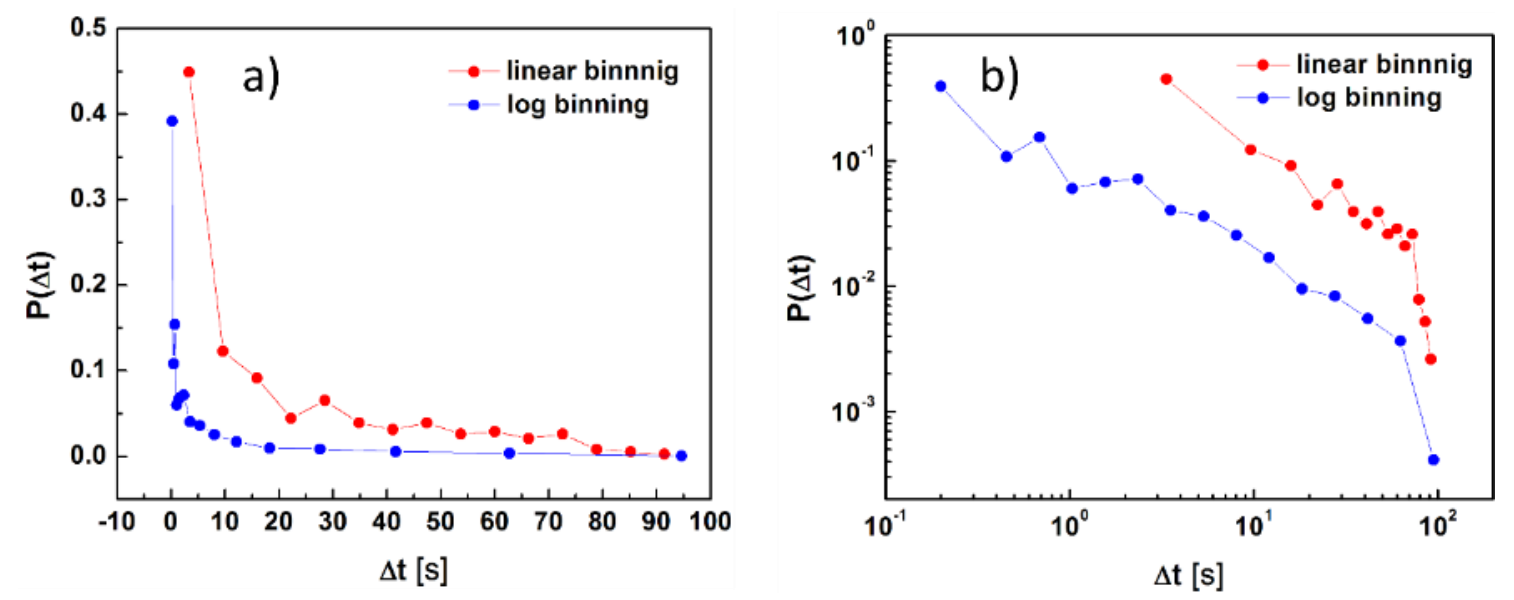

Figure 4.4. Comparison between linear and logarithmic binning. (a) Linear representation of the waiting time distribution $P(\Delta t)$ obtained from a Fe-rich SA2605 ribbon measured at $\sigma=15 \mathrm{MPa}$ and $\frac{T}{T_{g}}=0.85$, calculated with linear binning (red) and logarithmic binning (blue). (b) logarithmic scale representation of the waiting time distribution $P(\Delta t)$ obtained from the same experiment calculated with linear binning (red) and logarithmic binning (blue)

\subsubsection{Evaluation of power-law distributed data}

As described in theory section, one of the features of avalanching systems is the fact that the probability distribution of several relevant quantities scale as a power law, given by the following expression:

$$
P(S) \sim A S^{-\tau}
$$

Computing the logarithm of both side terms of the expression above leads to:

$$
\log (P(S)) \sim \log (A)-\tau \log (S)
$$

A standard approach to check whether the distribution of a given parameter scales as a power law, consists in the estimation of its probability and it subsequent representation in double-logarithmic scale. Such distribution is compatible with a power law, if the double-logarithmic plot scale shows linearity over a certain regime, and the exponent $\tau$ can be calculated from a linear regression in that regime. In order to perform a linear regression which the same weight over the whole fitting range, equidistant binning in the log-scale is required. This suggests the use of a logarithmic binned histogram for this kind of analysis.

It should be stressed, that the linearity in the double-log representation is a necessary but not sufficient condition for a power law distribution. In fact, many functions $P(S)$ may show a linear decay over a limited regime when plotted in double log representation, as long as $P(S)$ decreases 
sufficiently fast with the variable S. Thus, in order to support the hypothesis of power law scaling, it is important to check whether linearity is preserved over an extended range of values of the variable under study. In relevant literature, authors often talk about the decades of power law scaling. The number of decades of power law scaling in a certain data set turns out to be one of the strongest arguments to support the hypothesis of a power law probability distribution of such set.

In the theory chapter it was recalled that many of the usual power law distributions are expected to be truncated by an exponential cut-off. This cut-off may have different origins, according to the variable under study, in particular fine-size and finite time effects. The points belonging to the cutoff should not be used in the linear regression, in order to estimate accurately the exponent $\tau$.

\subsubsection{Estimation of crossover in the waiting time distribution}

Krisponeit et al reported a crossover in the statistics of the waiting times produced during a creep measurement of an amorphous $\mathrm{Pd}_{77.5} \mathrm{Cu}_{6} \mathrm{Si}_{16.5}$ ribbon measured at $\mathrm{T}=320^{\circ} \mathrm{C}$ and an applied stress of $\sigma=12 \mathrm{MPa}$ during one week ${ }^{30}$. According to their result, the waiting times measured until a certain time from the beginning of the experiment (estimated to be $t_{\text {cross }} \sim 100 \mathrm{~min}$ ), showed a power law distribution with an exponent $\tau \sim-1.5 \pm 0.1$. The distribution of the remaining of the waiting times (measured from $t=t_{\text {cross }}$ until the end of the experiment $t=1$ week) exhibited instead a power with an exponent $\tau \sim-0.8 \pm 0.1$.

In this work, the effect of the experimental conditions on such crossover are investigated. To do that, a precise definition for the figure of merit $t_{\text {cross }}$ is required. This is done by defining $t_{\text {cross }}$ as the time that divides the data in two subsets $\left(t<t_{\text {cross }}\right)$ and $\left(t>t_{\text {cross }}\right)$ in such a way that minimizes the error of the fit of the experimental waiting time distributions of both subsets $P_{1}\left(\Delta t \mid t<t_{\text {cross }}\right)$ and $P_{2}\left(\Delta t \mid t>t_{\text {cross }}\right)$ and an ideal power law distribution $P_{1} \sim \Delta t^{-1.5}$ and $P_{2} \sim \Delta t^{-0.8}$ respectively.

In order to calculate $t_{\text {cross }}$ the creep curve is divided in two subsets at different experimental times $t_{i}$. For each $t_{i}$ value, the squared error between $P_{1}\left(\Delta t \mid t<t_{i}\right)$ and $P_{2}\left(\Delta t \mid t>t_{i}\right)$ and ideal power laws fits $\widetilde{P}_{1} \sim \Delta t^{-1.5}$ and $\widetilde{P}_{2} \sim \Delta t^{-0.8}$ is calculated. In each iteration, the error value is calculated and $t_{i}$ is increased, ranging from zero to the total experimental times in intervals of one second. At the end of the loop, $t_{\text {cross }}$ is chosen as the time $t_{i}$ with the minimum error.

Figure 4.5 (a) shows the creep curve of $\mathrm{Pd}_{77.5} \mathrm{Cu}_{6} \mathrm{Si}_{16.5}$ sample measured with a tensile stress of $\sigma=25 \mathrm{MPa}$, and a temperature $\frac{T}{T_{g}}=0.95$. The crossover is represented with a coloured background. Figure 4.5 (b) shows the distribution of the waiting times, before the crossover, after the crossover and for the whole creep curve, measured for the aforementioned conditions. 
a)

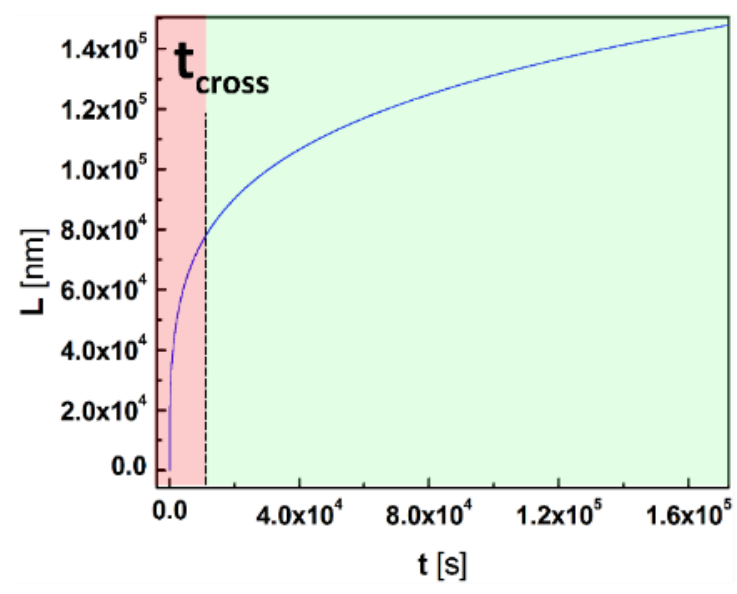

b)

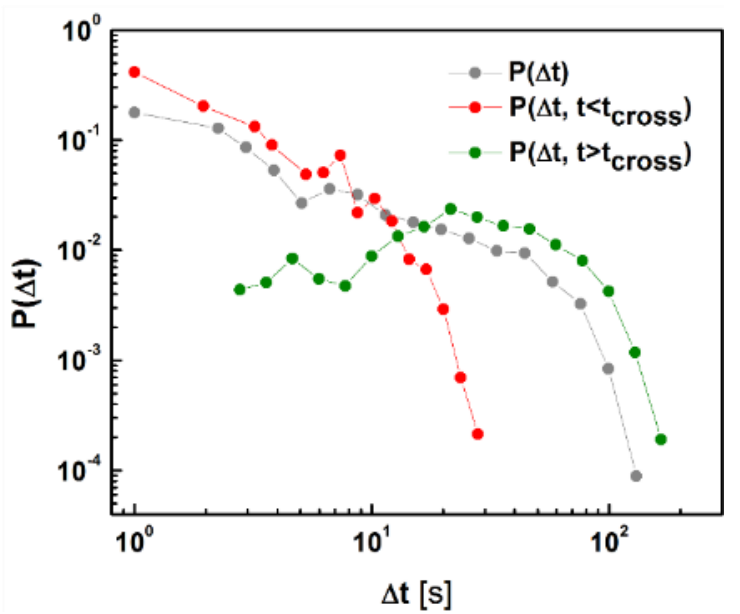

Figure 4.5 (a) Creep curve of a $\mathrm{Pd}_{77.5} \mathrm{Cu}_{6} \mathrm{Si}_{16.5}$ subjected to a tensile stress of $\sigma=$ $25 \mathrm{MPa} \frac{T}{T_{g}}=0.95$. The crossover is shown by the colored background and takes place at $t_{\text {cross }}=(1.64 \pm 0.6) \times 10^{4} s$. (b) Waiting time distribution before the crossover $P_{1}\left(\Delta t \mid t<t_{i}\right)$, after the crossover $P_{2}\left(\Delta t \mid t>t_{i}\right)$ and for the whole experimental time.

In order to estimate the error bars for the figure of merit $t_{\text {cross }}$, the same procedure was repeated for the exponents $\tau=1.5 \pm 0.2, \tau_{1},=-1.3, \tau_{1^{\prime \prime}}=-1.7$. The two crossover times obtained by the selection of $\tau_{1}$, and $\tau_{1^{\prime \prime}}$ are used as lower and higher end for the error bar $t_{\text {cross }}$. 


\subsubsection{Strain rate analysis}

Waiting times analysis, which focuses on the study of the intermittency in the creep signal in the context of the avalanche dynamics, has been described in former paragraphs. In addition, the creep measurements have been analyzed from the perspective of the average strain rate, as will be now discussed below.

To begin with, the intermittency from the creep curves was removed by applying a moving average filter to the creep curves. That process leads to smooth, differentiable curves, without any fine structure. Then, the average strain rate $\dot{\varepsilon}(t)$ and it derivative $\ddot{\varepsilon}(t)$ were calculated upon differentiation of the smoothed creep curves. Figure 4.6 displays the evolution of the strain and its two first derivatives in time $\dot{\varepsilon}(t)$ and $\ddot{\varepsilon}(t)$ as a function of time in a creep measurement performed on a $\mathrm{Pd}_{77.5} \mathrm{Cu}_{6} \mathrm{Si}_{16.5}$ ribbon subjected to a tensile stress of $\sigma=25 \mathrm{MPa}$ and a temperature of $\frac{T}{T_{g}}=$ 0.95 .
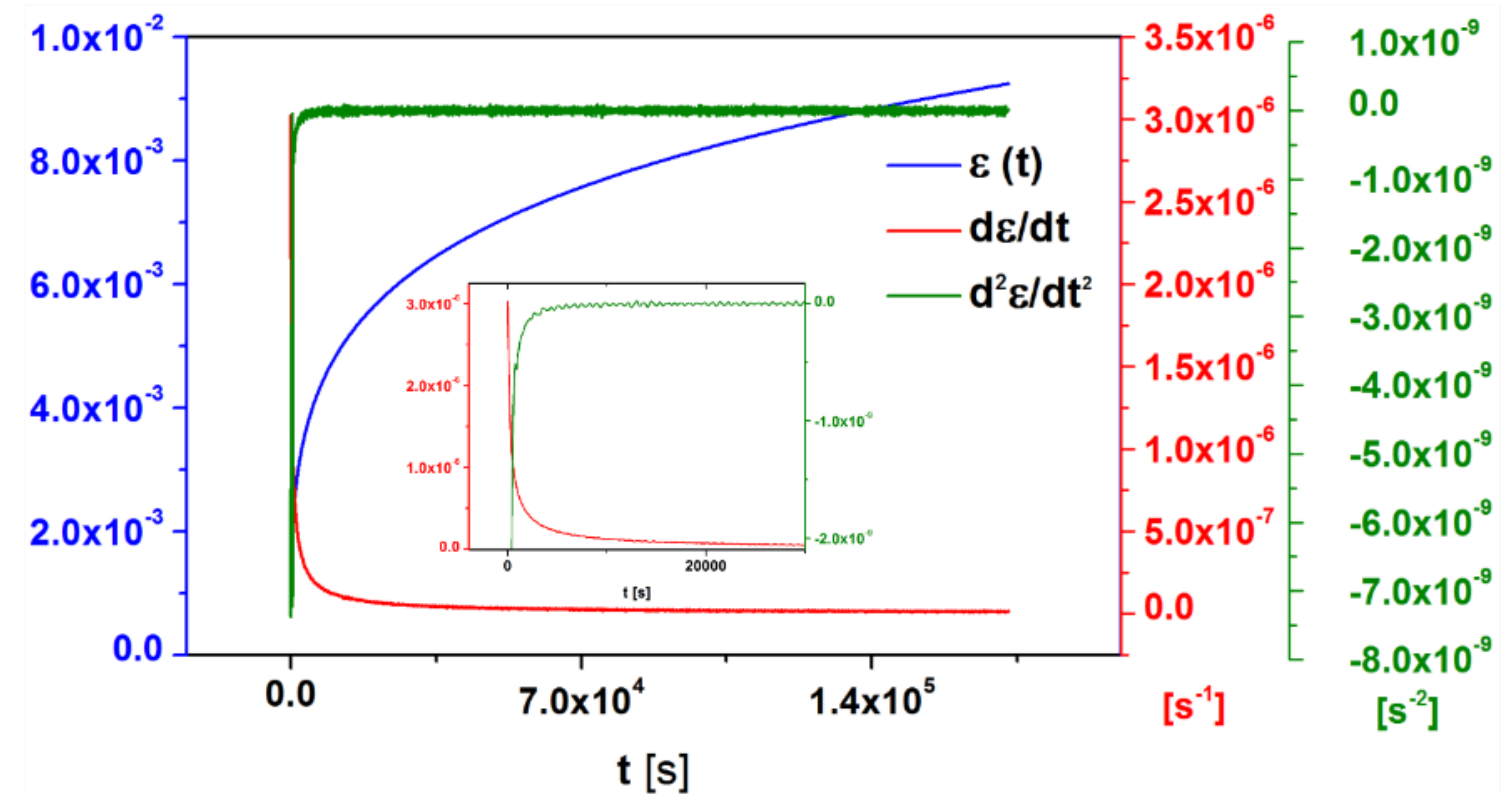

Figure 4.6 Evolution of the strain, strain rate, and second derivative of the strain as a function of the experimental time in a creep experiment performed in a $\mathrm{Pd}_{77.5} \mathrm{Cu}_{6} \mathrm{Si}_{16.5}$ ribbon subjected to a tensile stress of $\sigma=25 \mathrm{MPa}$ and a temperature of $\frac{\mathrm{T}}{\mathrm{T}_{\mathrm{g}}}=0.95$. The creep signal has been smoothed, applying a moving average filter with a window size of 100 seconds, in order to remove the fine structure of the data so that time derivatives could actually be computed. Inset: zoom of strain rate and second derivative of the strain at early times of the creep measurement.

Figure 4.6 shows that although the (filtered) strain and its derivatives are continuous functions, the average strain rate $\dot{\varepsilon}(t)$ decreases dramatically with time at early experimental times, to eventually reach an steady state value, characterized by almost zero-valued second derivative of the strain $\ddot{\varepsilon}(t)$. In fact, two regimes can now be distinguished, an initial transient, characterized by high values of the modulus of the strain rate $\dot{\varepsilon}(t)$, and it derivative $\ddot{\varepsilon}(t)$ and a second regime in which the strain rate approaches a constant value. 
A figure of merit $t_{\dot{\varepsilon}}$ is proposed to characterize such transition. Specifically, $t_{\dot{\varepsilon}}$ is defined as follows:

$$
t_{\dot{\varepsilon}}=\min (t) \quad \text { such that } \quad \varepsilon(t)<\alpha \varepsilon_{\text {steady-state }}
$$

Where $\varepsilon_{\text {steady-state, }}$ represents the average steady state strain rate, and $\alpha$ is an arbitrary proportionality constant. The procedure for estimating $t_{\dot{\varepsilon}}$ consists firstly in finding the experimental times $t_{\alpha_{1}}$ and $t_{\alpha_{2}}$ that fulfill the condition given by equation (4.7) for the values of alpha of $\alpha_{1}=20$ and $\alpha_{2}=10 . t_{\dot{\varepsilon}}$ is then defined as the average of those values and $t_{\alpha_{1}}$ and $t_{\alpha_{2}}$, that are also used to define the upper and lower limits of the error bars respectively. Figure 4.7 shows a closer look to the evolution of the strain rate as a function of time for the $\mathrm{Pd}_{77.5} \mathrm{Cu}_{6} \mathrm{Si}_{16.5}$ ribbon subjected to a tensile stress of $\sigma=25 \mathrm{MPa}$ and a temperature of $\frac{T}{T_{g}}=0.95$. The values $t_{\dot{\varepsilon}}, t_{\alpha_{1}}, t_{\alpha_{2}}$ are also shown in the graph.

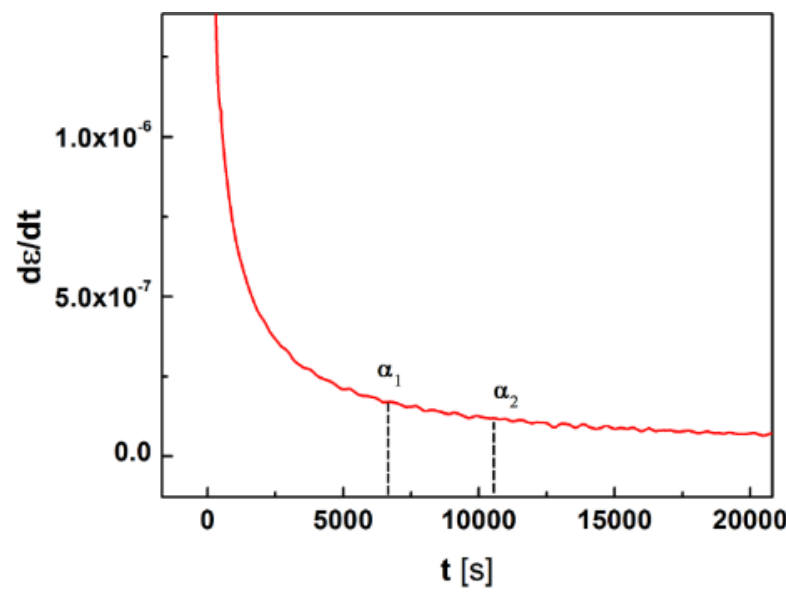

Figure 4.7. Strain rate as a function of time for a $\mathrm{Pd}_{77.5} \mathrm{Cu}_{6} \mathrm{Si}_{16.5}$ ribbon subjected to a tensile stress of $\sigma=25 \mathrm{MPa}$ and a temperature of $\frac{\mathrm{T}}{\mathrm{T}_{\mathrm{g}}}=0.95$. the crossover in strain rate $t_{\dot{\varepsilon}}$ is shown as well as its lower and higher bounds $t_{\alpha_{1}}$ and $t_{\alpha_{2}}$.

At this juncture, it should be observed that estimating $t_{\dot{\varepsilon}}$ in principle relies in the choice of the threshold above, which in turn depends on the values selected for the constants $\alpha_{1}$ and $\alpha_{2}$. It is shown in Appendix A that such choice does not qualitatively change the functional dependence of $t_{\dot{\varepsilon}}$ on temperature and stress. 


\subsubsection{Energy distribution analysis}

The creep measurements can be also analyzed from the point of view of the Potential Energy Landscape (PEL) that has been introduced in the theory section. In this framework, every change on the system's configuration can be associated to a transition between local minima in the PEL. Since a given waiting time $\Delta t$ is inversely proportional to the configuration change rate, using the Transition State Theory it can be associated to an effective energy barrier in the following way:

$$
\Delta t(t)=\Delta t_{0} e^{\left(\frac{E(t)}{K T}\right)}
$$

Where $E(t)$ represents the effective energy barrier that corresponds to the transition represented by the waiting time $\Delta t(t), \mathrm{T}$ is the temperature, $\mathrm{K}$ the Boltzmann constant and $\Delta t_{0}$ is a proportionality constant.

It is important to note than in the PEL picture the energy barriers are all static and do not evolve with time. However In equation (4.8) the time dependence on $\Delta t(t)$ and $E(t)$ was written to represent that the configuration state of the system does evolve with time, and through this evolution the system undergoes different energy barriers whose height depend on the current configurational state. From equation (4.8) the effective Energy barrier $E(t)$ can be estimated from a given waiting time $\Delta t(t)$, which is the measurable variable.

$$
E(t)=\mathrm{KT}\left[\log (\Delta t(t))-\log \left(\Delta t_{0}\right)\right]
$$

For every creep measurement the waiting times $\Delta t(t)$ as a function of the experimental time can be computed following the methods shown in section 4.1.1. Using Eq (4.9) the variable waiting time can be translated into the effective barrier $E(t)$. Notice that the constant $\log \left(\Delta t_{0}\right)$ is set to zero for simplicity, so all energy values are arbitrarily shifted by a constant amount. Figure 4.8(a) shows an example of creep measurement on 2605SA1 sample at stress $\sigma=15 \mathrm{MPa}$ and temperature $\mathrm{T} / \mathrm{T}_{\mathrm{g}}=0.95$. Figure $4.8(\mathrm{~b})$ displays the estimation of the energy barriers derived from equation (4.3).

It can be seen that while $E(t)$ increases as a function of time, it does not so monotonically, but rather shows a certain amount of dispersion. To measure quantitatively such dispersion, a Locally Estimated Scatter -plot Smoother (LOESS) fit was performed to the $E(t)$ vs $t$ plots. Being LOESS a non-parametric method, no particular mathematical function need to be assumed. Using the LOESS fit, the residuals can be calculated as the difference between the experimental and the fitted Energy values:

$$
\Delta E(t)=E(t)_{\exp }-E(t)_{f i t}
$$

Given the definition of the residuals described by equation (4.10) the parameter $\Delta t_{0}$ cancels out. 
To quantitatively characterize the dispersion of the Energy barriers, the probability distribution of the residuals $P(\Delta E)$ is calculated. The Full Width half Maximum (FWHM) of the energy residuals distribution will be the figure of merit for the analysis of the dispersion of the energy barriers during the creep measurements and will be denoted as $W$ in this memoir. Figure 4.8(c) displays the evolution of the Energy residuals $\Delta E(t)$ calculated from a creep measurement of 2605SA1 sample at stress $\sigma=15 \mathrm{MPa}$ and temperature $T / T_{g}=0.95$, and Figure 4.8 (d) represents the probability distribution of such residuals and the value of $W$ in eV.
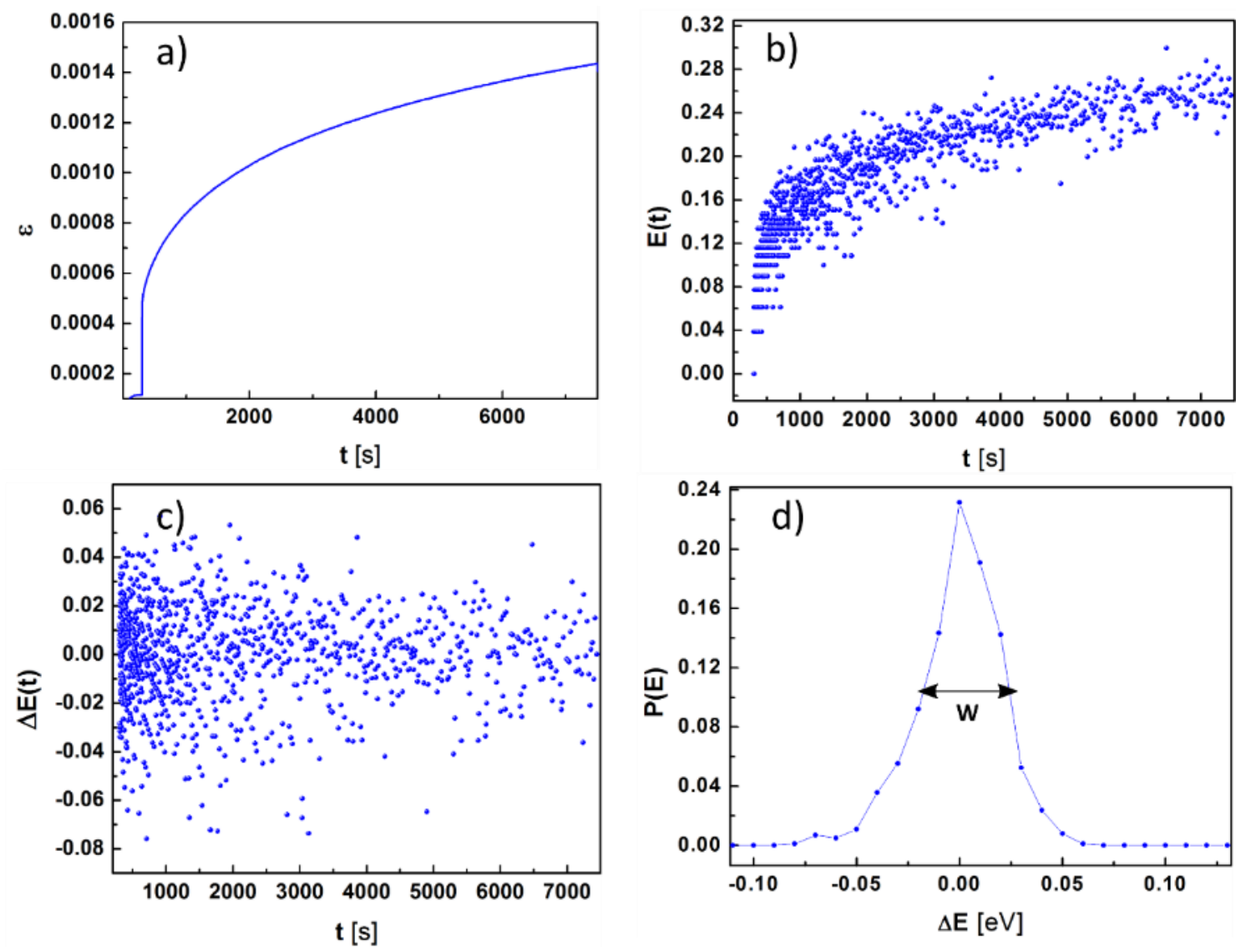

Figure 4.8 Energy dispersion analysis of a creep curve. (a) Creep curve of a Fe-rich 2605SA1 measured at stress $15 \mathrm{MPa}$ and temperature $\mathrm{T} / \mathrm{T}_{\mathrm{g}}=0.95$. (b) Scatter plot of the Energy barrier as a function of the experimental time. (c) Evolution of the Energy residuals, using a non-parametric LOESS fit on the scatter plot. (d) Probability distribution function of the distribution of residuals $P(\Delta E)$, the Full Width Half Maximum $(W)$ provides a quantitative measure of the dispersion in the scatter plot. 


\subsection{Analysis of stress-strain curves}

\subsubsection{The effective modulus analysis}

In section 3.5.4 the stress-strain test was introduced. Throughout this work all the stress-test measurements have been done in tensile geometry, and in a stress-controlled configuration of the DMA. Thus, the apparatus applies a stress ramp, with a constant stress rate $\dot{\sigma}$, and measures the tensile deformation exerted by the sample as a function of the applied stress at a given sampling rate. The sampling rate is synchronized to the stress rate, so the machine records the deformation response to every stress change.

A stress-strain curve is typically represented by plotting the elongation or strain on the horizontal axis (abscissa), and the applied stress on the vertical (ordinate) axis. However, in order to study the fine structure of the data, the resulting curve has to be analyzed with switched axis. This way the independent variable- the applied stress- is represented in the abscissa and the dependent variable and outcome of the experiments is exhibited in the ordinate, as it is shown in Figure 4.9 (a). A close look to the data represented this way, reveals an analogous fine structure as in the case of the creep measurements already discussed. Data are arranged in the shape of horizontal steps, equidistantly spaced in the vertical axis by the amount of $15 \mathrm{~nm}$. As already mentioned, the reason for this fine structure is the finite resolution of the apparatus, and therefore the same filtering method applies for the stress-strain measurement. Figure 4.9 (b) displays the fine structure of a stress-strain test, in which the staircase-like shape can be appreciated.

a)

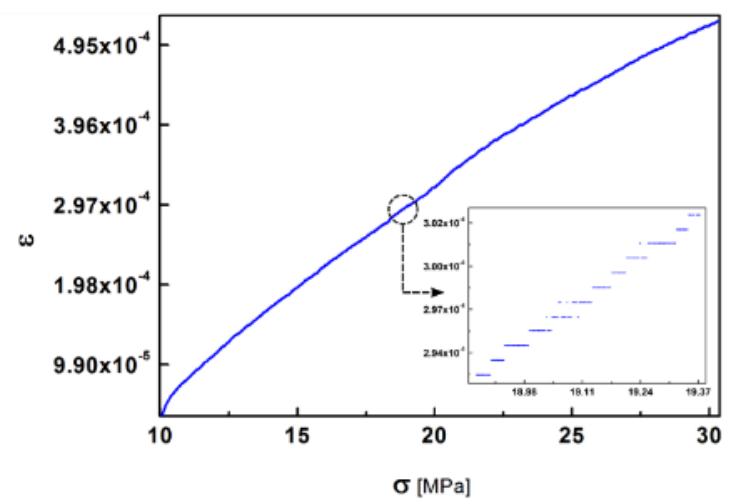

b)

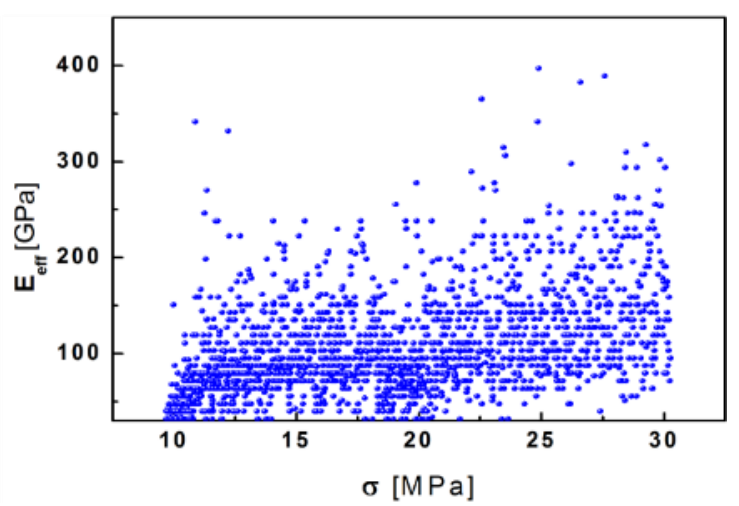

Figure 4.9 (a) strain versus stress measured in a stress ramp conducted on a Fe-rich 2605SA1 ribbon at $\mathrm{T}=30^{\circ} \mathrm{C}$ with a stress rate of $1.25 \times 10^{-2} \mathrm{MPa} / \mathrm{s}$ from $\sigma=10-$ $30 \mathrm{MPa}$. The inset reveals a fine structure of the data, which motivates the definition of the effective modulus. (b) Scatter plot representing the evolution of the effective modulus with respect to the applied stress for the stress-strain curve shown in Figure 4.9 (a)

However, in this case the physical meaning of the horizontal steps is different. In the case of the stress-strain test they represent the total amount of the stress increment necessary to deform the sample beyond the resolution limit. Since the magnitude of the deformation jumps is constant (and 
given by the spatial resolution of $15 \mathrm{~nm}$ of the machine), each stress interval can be associated to a region of an effectively constant Young Modulus in a coarse grained approximation. Such quantity is given by:

$$
E_{e f f}(t)=\frac{\Delta \sigma(t)}{\Delta \varepsilon}
$$

It should be pointed, that this analysis do not imply claiming that the actual deformation behavior of the sample is purely elastic at any given time. Instead, the definition of $E_{e f f}$ is constrained by the fine structure of the data. Such effective modulus includes the contribution of all the elastic and plastic deformation events that may occur in the vicinity of a time $t$ that cannot be told apart due to the limits in the experimental resolution of the apparatus. When measured with an oscillatory force and with a resolution high enough to measure accurately small phase angles between strain and stress, those elastic and plastic events would contribute to the storage and loss modulus.

Through the aforementioned analysis, from each stress-strain measurement the effective modulus of the mechanical response $E_{e f f}$ can be calculated as a function of the driving stress. Figure 4.9 (b) displays the scatter plot of the $E_{\text {eff }}(t)$ as a function of the applied stress calculated from the stressstrain test shown in Figure 4.9 (a).

The probability distribution of the effective modulus can be calculated by means of a histogram, as it was discussed in previous sections. Figure 4.10 shows the probability distribution of the effective modulus for the Fe-rich $2605 \mathrm{SA} 1$ ribbon at $\mathrm{T}=30^{\circ} \mathrm{C}$ with a stress rate of $1.25 \times 10^{-2} \mathrm{MPa} / \mathrm{s}$.

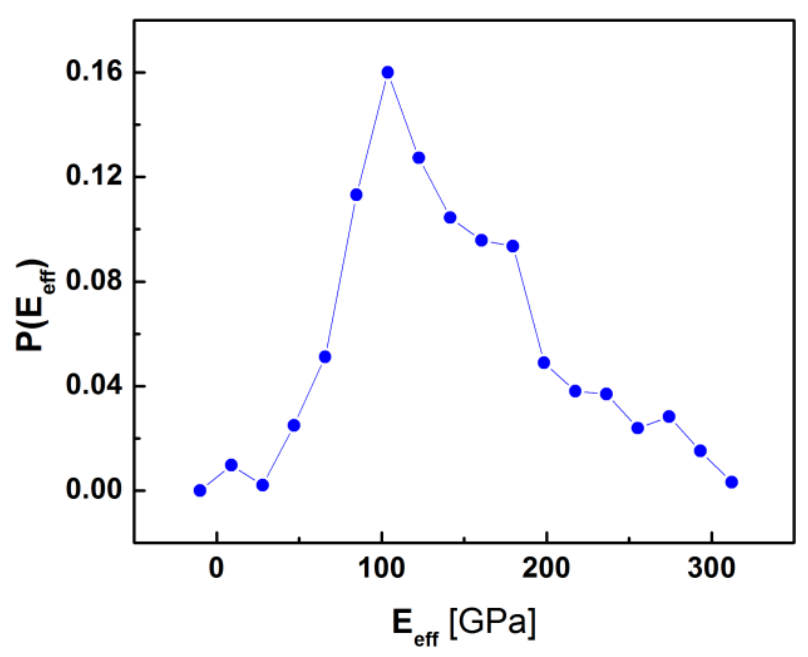

Figure 4.10 Probability distribution function of the effective modulus measured from a stress-strain experiment of Fe-rich $2605 \mathrm{SA} 1$ ribbon at $\mathrm{T}=30^{\circ} \mathrm{C}$ with a stress rate of $1.25 \times 10^{-2} \mathrm{MPa} / \mathrm{s}$ from $\sigma=10-30 \mathrm{MPa}$. 


\subsubsection{Avalanche analysis in stress-strain curves: the renormalized modulus}

To analyze the data in terms of the avalanche behavior a renormalization of the effective modulus $E_{e f f}(t)$ is proposed. Such normalization is made by computing the ratio of the effective modulus $E_{e f f}(t)$ to the global macroscopic modulus of each of the experiments $E_{\text {macro }}$, which is calculated from a linear fit of the stress-strain curve. The global macroscopic modulus $E_{\text {macro }}$ does not include any information regarding the intermittency of the data and provides a description of the averaged linear behavior.

The normalized modulus $\hat{E}(t)=\frac{E_{\text {macro }}}{E_{\text {eff }}(t)}$ gives an insight of the deviation from the linear behavior. In metallic glasses, such deviations are expected to be caused by a wider distribution of local elastic properties compared to the crystalline materials ${ }^{61}$, as well as by the mechanical avalanches. As discussed in the theory chapter, in a stress-controlled experiment, mechanical avalanches produce an instantaneous strain burst in the material, which would trigger an instantaneous decrease of the effective modulus $E_{\text {eff }}$. Therefore, the definition of $\hat{E}$ is useful to identify the avalanches by means of the condition $\hat{E}>1$, the normalized modulus value being proportional to the amount of strain carried out by those avalanches. Figure 4.11 displays the probability distribution function of the normalized modulus $P(\hat{E})$ calculated in a strain stress test of a 2605SA1 ribbon at $\mathrm{T}=300 \mathrm{~K}$ with a stress rate of $1.25 \times 10^{-2} \mathrm{MPa} / \mathrm{s}$. It can be observed that the distribution is centered around the value $\hat{E}=1$, which would correspond to a perfectly linear behavior, but there are few events which fulfil the condition $\hat{E}>1$, that characterize the strain bursts or avalanches.

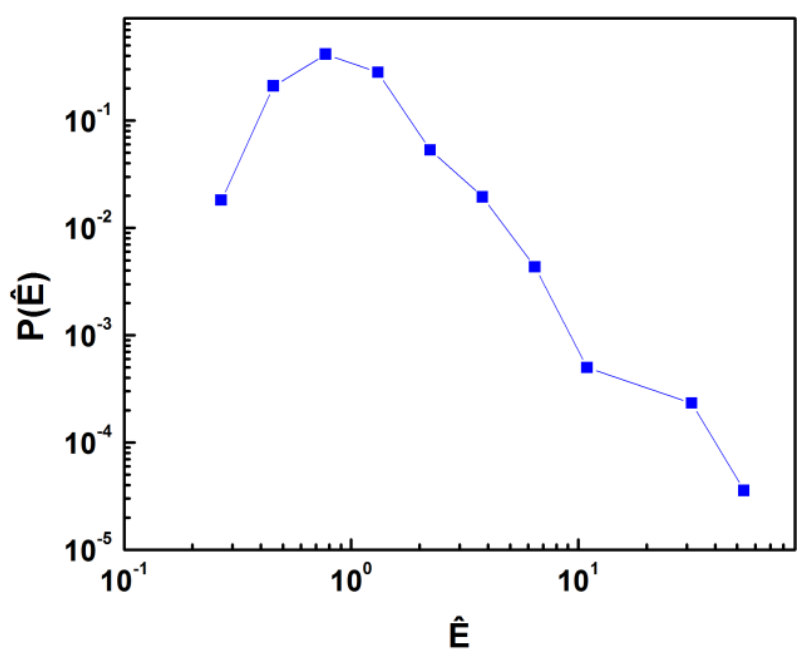

Figure 4.11 Probability distribution of the renormalized modulus $P(\widehat{E})$ calculated from a stressstrain experiment of Fe-rich 2605SA1 ribbon at $\mathrm{T}=300 \mathrm{~K}$ with a stress rate of $1.25 \times 10^{-2} \mathrm{MPa} / \mathrm{s}$ from $\sigma=10-30 \mathrm{MPa}$. 


\section{$5 \quad$ Results}

In this chapter, the experimental results are presented and analyzed according to the methods introduced in section 4 .

Subsection 5.1 focuses on the quantitative study of creep curves of a non-magnetic $\mathrm{Pd}_{77.5} \mathrm{Cu}_{6} \mathrm{Si}_{16.5}$ alloy. The experimental data is analyzed from the point of view of the avalanche regime and the macroscopic strain rate, making use of the figures of merit $t_{\text {cross }}$ and $t_{\dot{\varepsilon}}$. The role played by the experimental parameters stress and temperature on the avalanche behaviour and macroscopic strain rate is described and briefly discussed.

In Subsection 5.2, creep curves of a Fe-rich 2605SA1 alloy under simultaneous stress and magnetic field driving are analyzed. The experimental results are organized in several subsections. Each of them focuses on the influence of the different experimental parameters: Temperature, stress, magnetic field intensity and magnetic field orientation around two axes of symmetry.

Finally, in subsection 5.3 room temperature stress-strain measurements of magnetic alloys under magnetic field driving are shown. Mechanical tests were perfomed on two different compositions: highly magnetostrictive Fe-rich 2605SA1, and low magnetostrictive $\mathrm{Fe}_{2} \mathrm{Co}_{73} \mathrm{Si}_{10} \mathrm{~B}_{15}$ alloy. The effect of the magnetic field on both the macroscopic behavior and the intermittency in the deformation is analayzed. 


\subsection{Creep deformation of a non-magnetic sample}

The aim of the experiments shown in this subsection is to study the effect of the stress and temperature on the avalanche behavior of a $\mathrm{Pd}_{77.5} \mathrm{Cu}_{6} \mathrm{Si}_{16.5}$ metallic glass. In order to do that, several creep experiments were done under different conditions of stress and temperature. All the experiments were performed on freshly prepared $40 \mu \mathrm{m}$ thick, $2 \mathrm{~mm}$ width and $15 \mathrm{~mm}$ long ribbons prepared by melt spinning technique according to section 3.1 .

Prior to the creep measurements, the element composition of the alloy was analyzed by means of dispersive X-rays. Figure 5.1 shows the EDX spectrum, in which the spectral lines of $\mathrm{Pd}, \mathrm{Cu}$ and $\mathrm{Si}$ stand out clearly from the noisy background. Table 5.1 displays the atomic percentage of the elements calculated from the quantitative analysis of such spectrum. The element percentage shown in Table 5.1 confirms that the alloy preserved the nominal composition $\operatorname{Pd}_{77.5} \mathrm{Cu}_{6} \mathrm{Si}_{16.5}$ after the whole fabrication process.

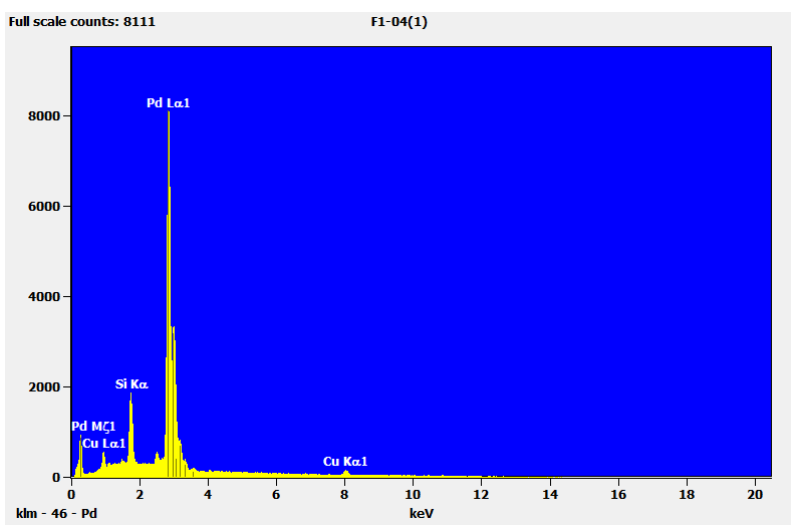

Figure 5.1 EDX spectrum of $\mathrm{Pd}_{77.5} \mathrm{Cu}_{6} \mathrm{Si}_{16.5}$ alloy

Table 5.1 Quantitative analysis of the EDX spectrum of $\mathrm{Pd}_{77.5} \mathrm{Cu}_{6} \mathrm{Si}_{16.5}$

\begin{tabular}{|c|c|}
\hline Element & Atomic percentage [\%] \\
\hline $\mathrm{Pd}$ & $78.1 \pm 0.6$ \\
\hline $\mathrm{Cu}$ & $6.2 \pm 0.8$ \\
\hline $\mathrm{Si}$ & $15.7 \pm 0.3$ \\
\hline
\end{tabular}

The thermal properties of the alloy, such as the crystallization and glass transition temperatures $\mathrm{T}_{\mathrm{X}}$ and $\mathrm{T}_{\mathrm{g}}$, were also measured prior to the creep test, in order to choose the temperature range of the experiments. $\mathrm{T}_{\mathrm{X}}$ and $\mathrm{T}_{\mathrm{g}}$ were measured through a DSC scan performed with a heat rate of $20 \mathrm{~K} / \mathrm{min}$. According to the DSC scan shown in Figure 5.2 the glass transition and crystallization temperatures of the alloy are estimated to be: $\mathrm{T}_{\mathrm{g}}=621 \pm 10 \mathrm{~K}$ and $\mathrm{T}_{\mathrm{X}}=685 \pm 10 \mathrm{~K}$. 


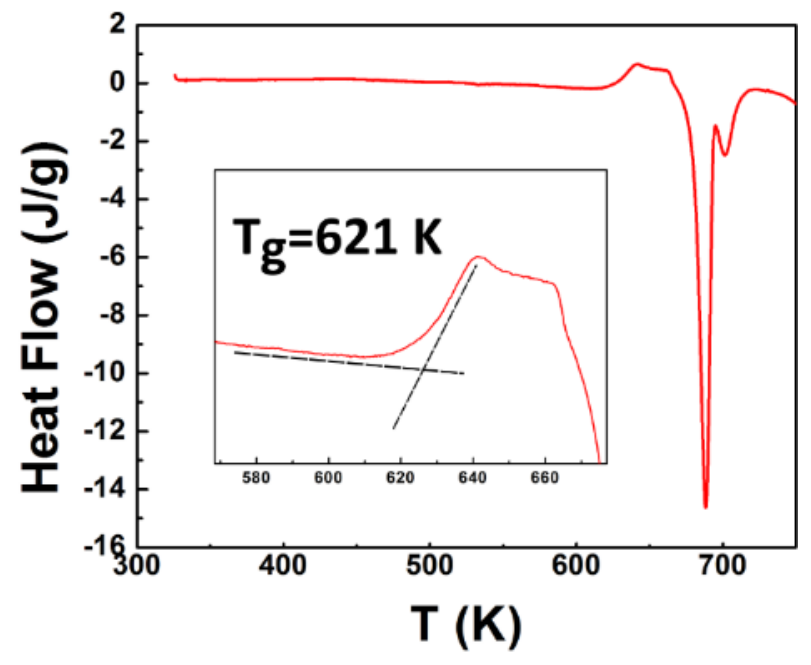

Figure $5.2 \mathrm{DSC}$ scan of $\mathrm{Pd}_{77.5} \mathrm{Cu}_{6} \mathrm{Si}_{16.5}$ measured with a heat rate of $\dot{\mathrm{T}}=20 \mathrm{~K} / \mathrm{min}$. The glass transition and crystallization temperatures can be estimated from the heat hump and crystallization peaks respectively: $\mathrm{T}_{\mathrm{g}}=621 \pm 10 \mathrm{~K}$ and $\mathrm{T}_{\mathrm{X}}=$ $685 \pm 10 \mathrm{~K}$

The structure of the samples was analyzed prior and after the creep tests by means of X-Ray Diffraction experiments. The analysis of the As-cast material allows to ensure that the samples are amorphous before the mechanical test, and therefore is a basic test to check the validity of the fabrication method. The analysis of the samples after being tested, permit to check that the sample did not crystallized nor oxidized during the measurement. Figure 5.3 displays a sample of the XRD spectra of the samples measured in this section. All the measurements show the characteristic amorphous halo, which is the signature of the amorphous state and the lack of crystalline phase. It can be seen that even for the measurements performed at the higher temperatures and mechanical loads the structure remains amorphous after the test. The inset shows that for the highest temperature $\left(\frac{\mathrm{T}}{\mathrm{T}_{\mathrm{g}}}=0.97\right)$ the amorphous halo was punctuated by two crystallization peaks which disappeared after polishing the sample. That is a signature of a small amount of surface oxidation, in which the bulk is still amorphous. Therefore, that sample was taken into account for the analysis. 


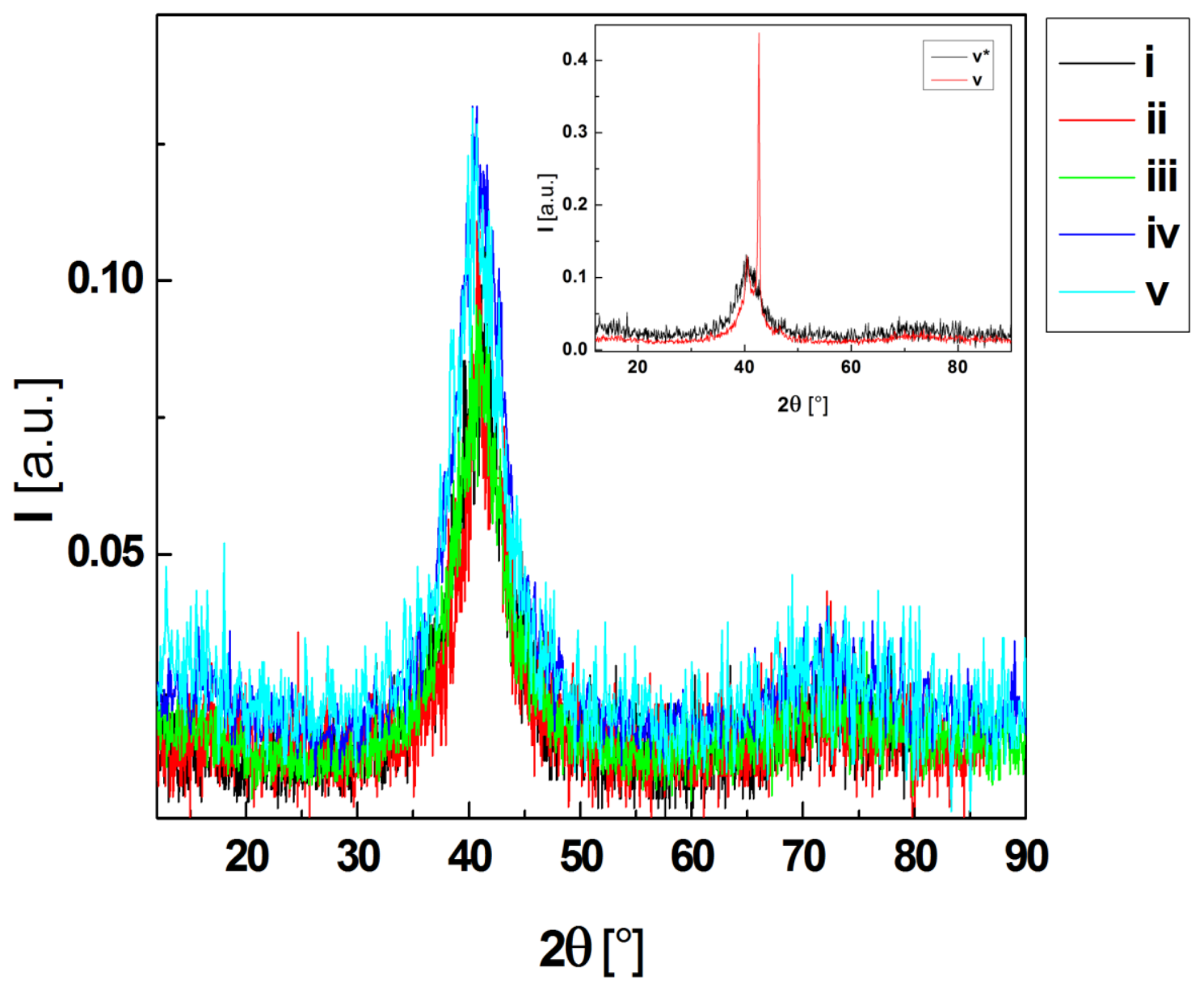

Figure 5.3 XRD spectra of $\mathrm{Pd}_{77.5} \mathrm{Cu}_{6} \mathrm{Si}_{16.5}$ samples. i) As cast. ii) Creep at $\frac{\mathrm{T}}{\mathrm{T}_{\mathrm{g}}}=$ $0.8, \sigma=12$. iii) Creep at $\frac{\mathrm{T}}{\mathrm{T}_{\mathrm{g}}}=0.89, \sigma=12$. iv) Creep at $\frac{\mathrm{T}}{\mathrm{T}_{\mathrm{g}}}=0.94, \sigma=36 \mathrm{MPa}$. v) Creep at $\frac{\mathrm{T}}{\mathrm{T}_{\mathrm{g}}}=0.97, \sigma=12 \mathrm{MPa}$. Inset: $\mathrm{V}^{*}$ ) Creep at $\frac{\mathrm{T}}{\mathrm{T}_{\mathrm{g}}}=0.97, \sigma=12 \mathrm{MPa}$ before polishing

After the sample characterization, a first set of creep tests was conducted to check if the crossoverreported by Krisponeit et al., ${ }^{30}$ take place for a wide range of experimental conditions. Thus, creep experiments were performed at stress and temperaturas in the range $\sigma=\{2-12\} \mathrm{MPa}$ and $\frac{T}{T_{g}}=\{0.85-0.94\}$. In each of the experiments a freshly prepared sample from the very same sample batch was subjected to the corresponding constant stress and temperature during a week. An overview of these creep experiments is given in Figure 5.4, which presents the creep curves measured at $\sigma=2,8$ and $12 \mathrm{MPa}$ for each of the temperatures $\frac{T}{T_{g}}=0.85,0.89,0.92$ and 0.94 


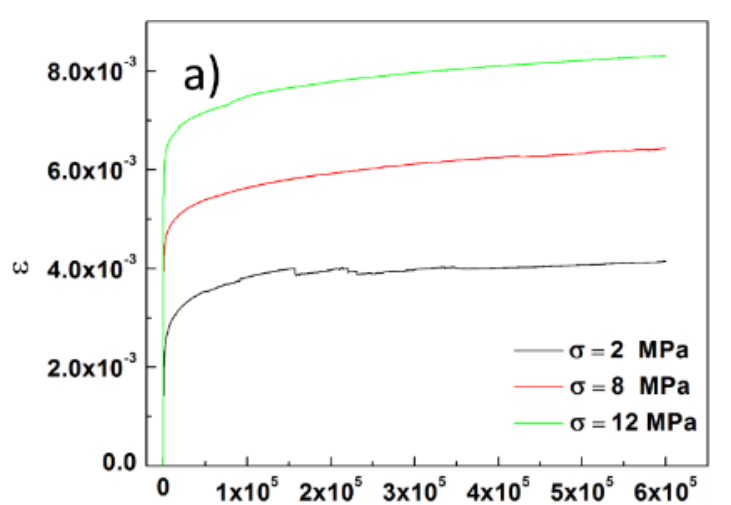

$\mathrm{t}[\mathrm{s}]$

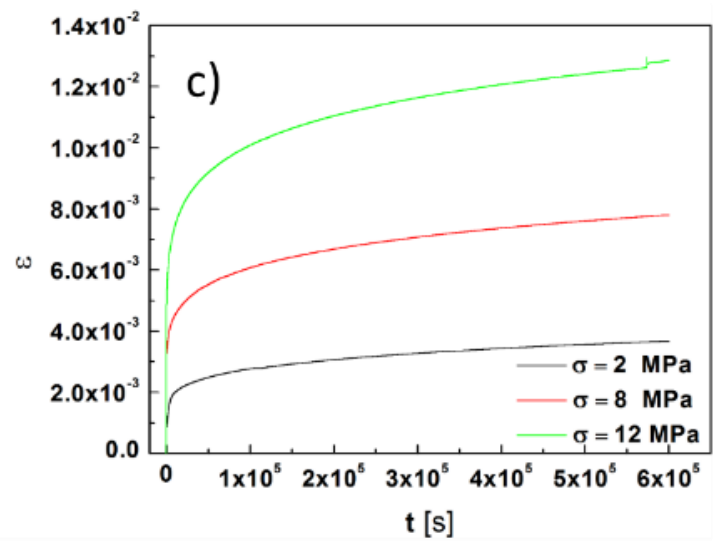

b)

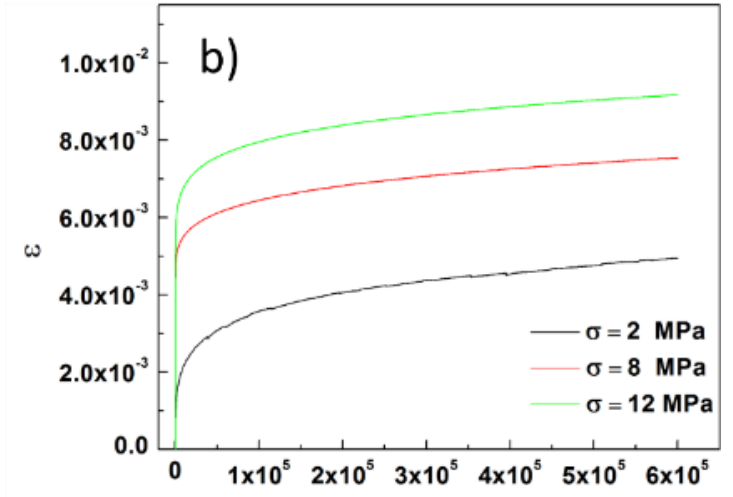

$t[s]$

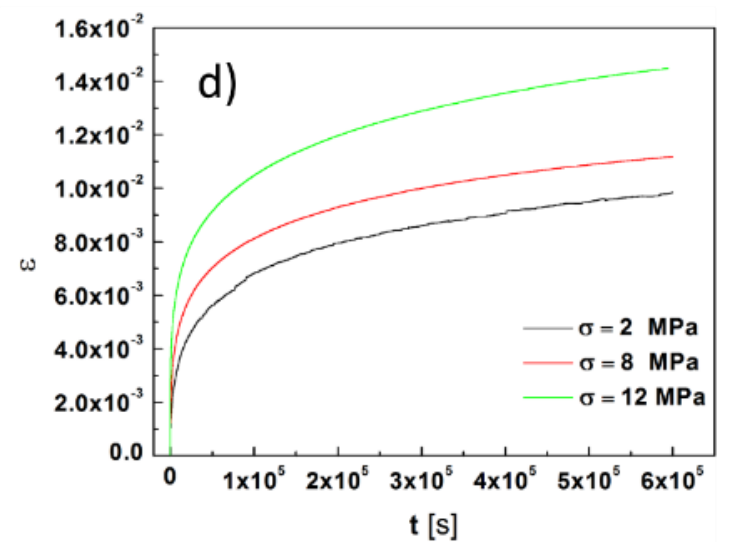

Figure 5.4 (a) Creep curves at $\frac{\mathrm{T}}{\mathrm{T}_{\mathrm{g}}}=0.85$ and $\sigma=2 \mathrm{MPa}$ (black line), $\sigma=8 \mathrm{MPa}$ (red line), and $\sigma=12 \mathrm{MPa}$ (green line). (b) Creep curves at $\frac{\mathrm{T}}{\mathrm{T}_{\mathrm{g}}}=0.89$ and $\sigma=2$ $\mathrm{MPa}$ (black line), $\sigma=8 \mathrm{MPa}$ (red line), and $\sigma=12 \mathrm{MPa}$ (green line). (c) Creep curves at $\frac{\mathrm{T}}{\mathrm{T}_{\mathrm{g}}}=0.92$ and $\sigma=2 \mathrm{MPa}$ (black line), $\sigma=8 \mathrm{MPa}$ (red line), and $\sigma=12$ $\mathrm{MPa}$ (green line). (d) Creep curves at $\frac{\mathrm{T}}{\mathrm{T}_{\mathrm{g}}}=0.94$ and $\sigma=2 \mathrm{MPa}$ (black line), $\sigma=8$ $\mathrm{MPa}$ (red line), and $\sigma=12 \mathrm{MPa}$ (green line.) 
The fine structure of the creep measurements presented in Figure 5.4 was analyzed using the waiting time analysis, and the crossover was estimated following the method described in Chapter 4. The waiting time distribution for each of the experiments shown in Figure 5.4 is presented in Figure 5.5, shown below. That figure is arranged in a stress-temperature map fashion. The distribution shown in each square corresponds to the creep test performed with the stress and temperature values that corresponds to its position in such stress-temperature map. The background color represents the magnitude of the figure of merit $t_{\text {cross }}(\sigma, T)$ as indicated in the legend on the right hand side.

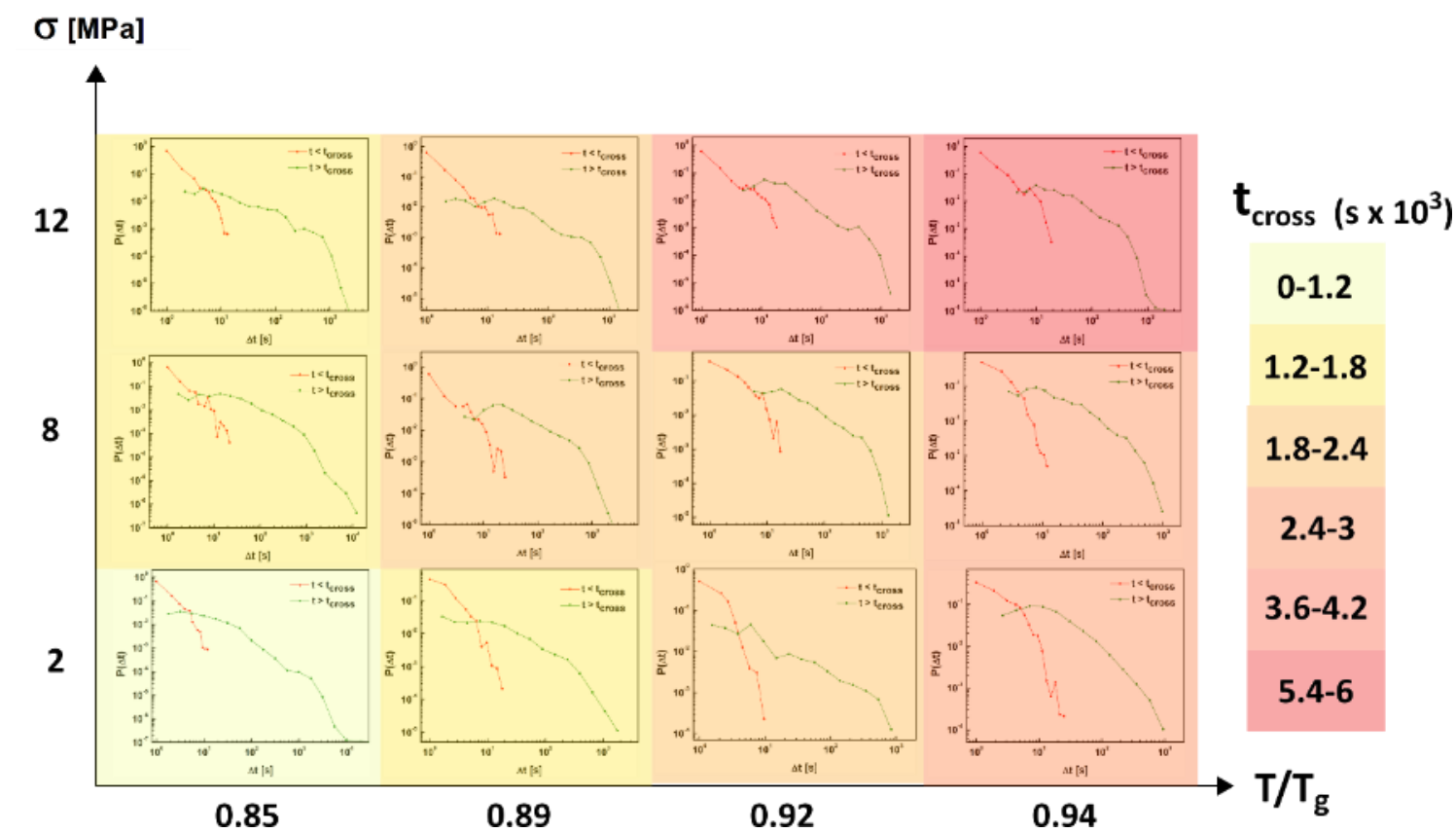

Figure 5.5 Stress-Temperature map. Each square represents the waiting time distribution before and after the crossover time $t_{\text {cross }}$ under the stress and temperature that corresponds with it position. The background color describes the magnitude of $t_{\text {cross }}{ }^{35}$.

Figure 5.5 shows that the waiting time distribution shows a crossover for a wide range of experimental conditions. Moreover, in Figure 5.6 it can be seen how the exponent of the waiting time distribution before and after the crossover is substantially different for each of the experimental conditions under analysis. The error bars and the dispersion in the data shown in Figure 5.6 implies that the exponent of the second regime $\tau_{2}$ remains fairly constant among all the experiments, with a value $\tau_{2}=0.8 \pm 0.1$ whereas the first regime exponent $\tau_{1}$ shows a higher amount of dispersion. Yet for the majority of the cases the fitted value is compatible with $\tau_{1}=$ -1.5 within the error bars. 


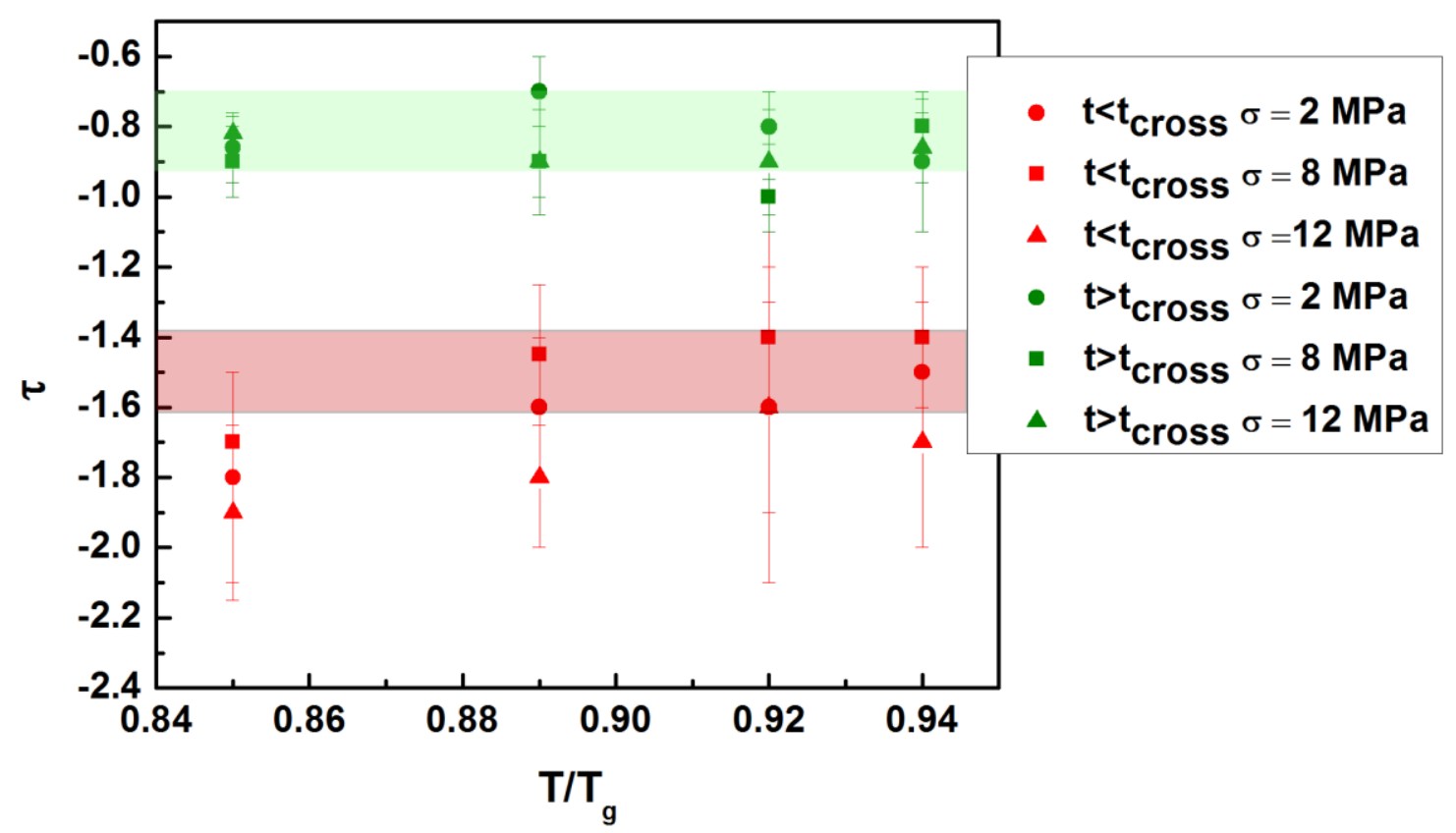

Figure 5.6 Exponents of the waiting time distributions in the stress-temperature map. Red symbols represents the exponent of the distribution before the crossover and green symbols correspond to the distribution after the crossover. The dashed lines represent the intervals $\tau_{1}=-1.5 \pm 0.1$ and $\tau_{2}=-0.8 \pm 0.1$.

From Figure 5.5 it can be also inferred that the crossover time increases both with the applied stress and temperature. In order to analyze in detail the dependence of $t_{\text {cross }}$ with the stress and temperature, more experiments were done to explore further the $\sigma-T$ space by adding two sets of experiments to the results of Figure 5.5. In the first set, the stress was kept constant ( $\sigma=12 \mathrm{MPa})$ among the different experiments and the temperature was swept through the range $\frac{T}{T_{g}}=0.67-$ 0.98 . In the second set, the temperature was kept constant $\frac{T}{T_{g}}=0.94$, the stress was swept in the range $\sigma=2-35 \mathrm{MPa}$, which corresponds to $\frac{\sigma}{E}=(0.05-0.6) \times 10^{-3}$, normalizing by the Young modulus. 

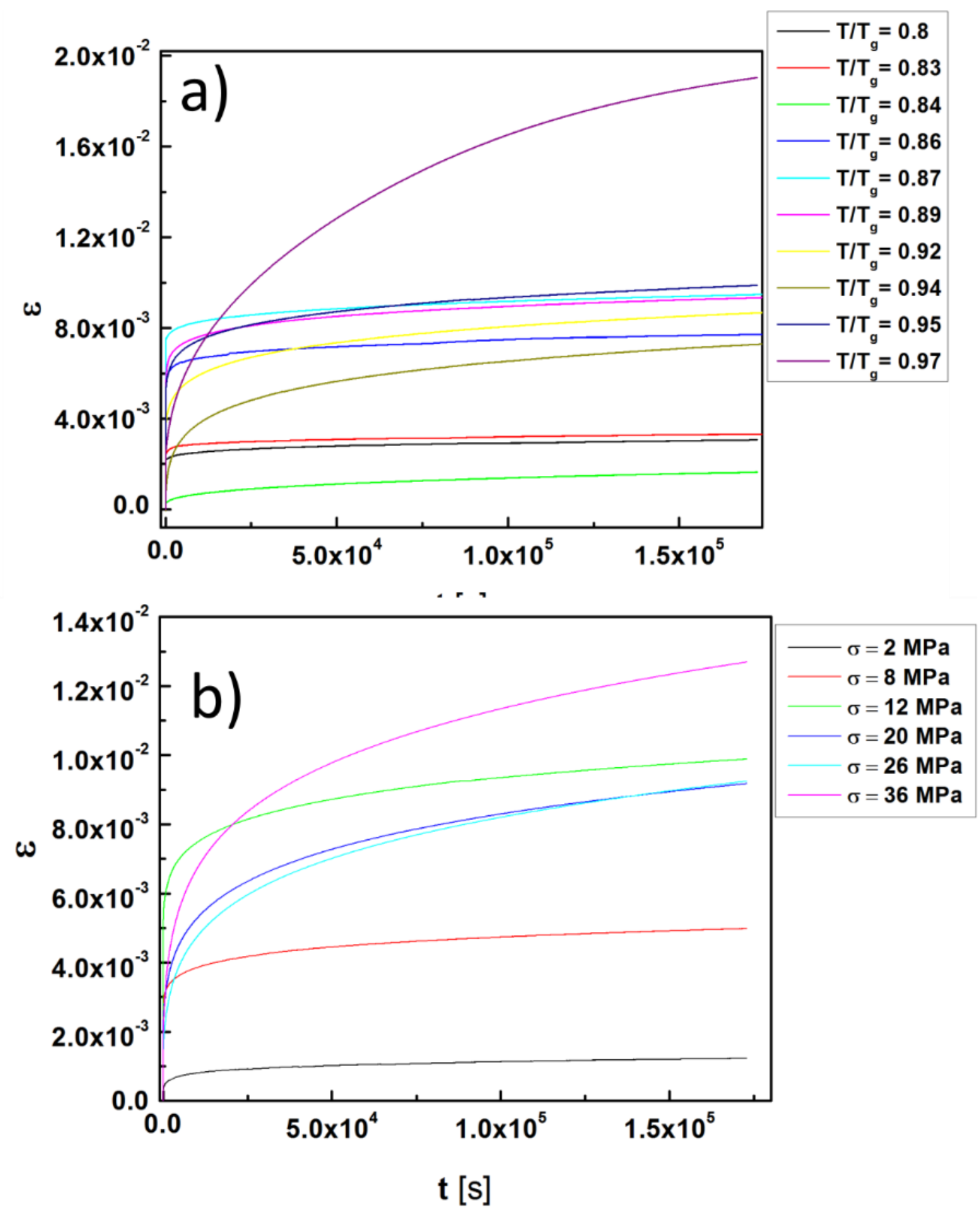

Figure 5.7 Creep measurements used to investigate the quantitative dependence of $t_{\text {cross }}$ with the stress and temperature. (a) Tests done keeping a constant stress $\sigma=12 \mathrm{MPa}$ and sweeping the temperature in the range $\mathrm{T} / \mathrm{T}_{\mathrm{g}}=0.8-0.97$. (b) Set of measurements performed keeping aconstant temperature $\mathrm{T} / \mathrm{T}_{\mathrm{g}}=0.94$ and sweeping the stress in the range $\sigma=2-36 \mathrm{MPa}$.

Additionally, the creep curves are analyzed from the point of view of the strain rate. According to the macroscopic strain rate, the curves can be divided in two regimes: A transient characterized by high values of the strain rate and its derivative and a second regime in which the strain rate approaches a steady state value. The experimental time in which this transition takes place, $t_{\dot{\varepsilon}}$, is 
estimated using the method described in Chapter 4. The evolution of $t_{\text {cross }}$ and $t_{\dot{\varepsilon}}$ as a function of the stress and temperature is depicted in Figure 5.8 shown below.
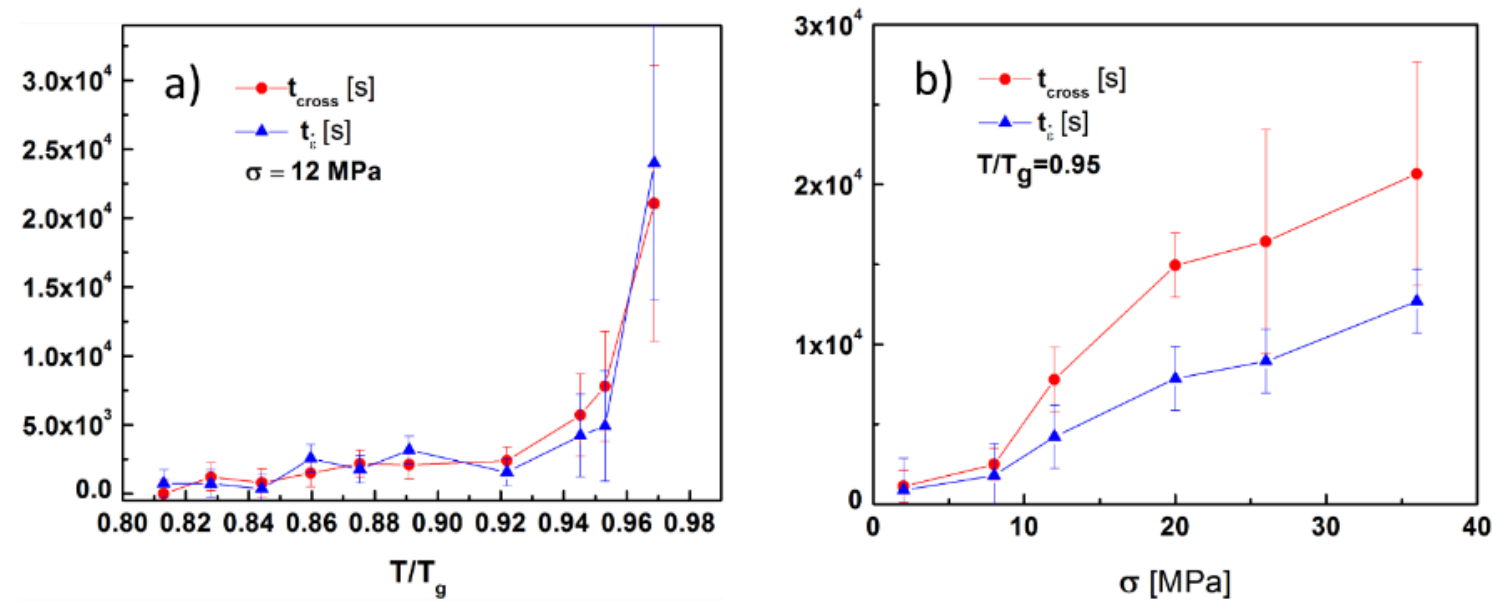

Figure 5.8 (a) Dependence of $t_{\text {cross }}$ (red) and $t_{\dot{\varepsilon}}$ (blue) with the temperature at a fixed stress. (b) Dependence of $t_{\text {cross }}$ (red) and $t_{\dot{\varepsilon}}$ (blue) with the stress at a fixed temperature ${ }^{35}$.

Figure 5.8 shows that there is a clear correlation between $t_{\text {cross }}$ and $t_{\dot{\varepsilon}}$ since both quantities show the same functional dependence with the stress and temperature. That result implies that the macroscopic strain rate is correlated to the avalanche regime or vice-versa, as has been reported in several simulations ${ }^{154}$ and experiments ${ }^{166}$. In addition both figures of merit present different functional dependence with the temperature and stress. This result suggests a different role of the temperature and stress in the deformation process. These results are interpreted and discussed in detail in Section 6. 


\subsection{Creep deformation of a magnetic glass under stress and magnetic driving}

In this subsection the role of the magnetic field in the deformation process is analyzed through the study of creep experiments carried out on Fe-rich 2605SA1 metallic glass. Different set of experiments were performed in order to focus on the influence of the temperature, stress and magnetic field separately. Also, to analyze in detail the influence of the magnetetoelastic coupling, the magnetic field analysis is divided in three different sections: The study of the field intensity (under two different mechanical stresses), the analysis of the influence of the azimuthal angle between the magnetic field and the width axis $\theta$, and the influence of the polar angle between the magnetic and mechanical fields $\phi$.

\subsubsection{Sample characterization}

The element distribution of the 2605SA1 sample was studied by means of dispersive X-ray spectroscopy (EDX). Figure 5.9 shows the EDX spectrum, in which Fe, Si and C lines can be clearly seen. Table 5.2 provides the quantitative analysis from that spectra, which allows to estimate the atomic percentage of each element. From Table it can be inferred that the magnetism of the 2605SA1 alloy, comes uniquely from the iron atoms.

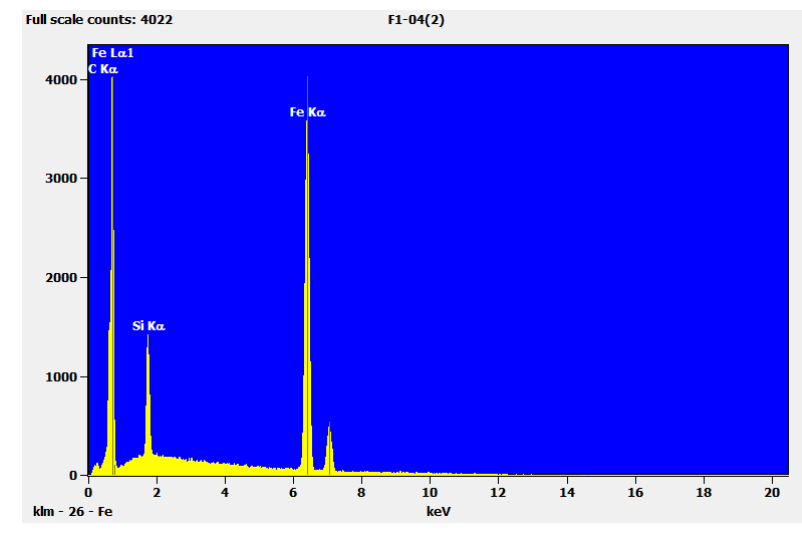

Figure 5.9 EDX pattern of the 2605SA1 alloy. The dispersive X-ray shows emission lines corresponding to $\mathrm{Fe}$, $\mathrm{Si}$ and $\mathrm{C}$

Table 5.2 Quantitative analysis of the EDX spectrum of 2605SA1

\begin{tabular}{|c|c|}
\hline Element & Atomic percentage \% \\
\hline $\mathrm{Fe}$ & $80.5 \pm 0.6$ \\
\hline $\mathrm{Si}$ & $8.4 \pm 0.1$ \\
\hline $\mathrm{C}$ & $11.1 \pm 2.2$ \\
\hline
\end{tabular}


The thermal properties of the metallic glass were characterized by Differential Scanning Calorimetry (DSC) as introduced in section 3. Figure 5.10 shows the result of the DSC scan, measured with a heat rate of $\dot{\mathrm{T}}=20 \mathrm{~K} / \mathrm{min}$. It shows a clear crystallization peak which takes place at $\mathrm{T}_{\mathrm{X}}=782 \pm 10 \mathrm{~K}$. Also, the inset of the figure shows that the Curie transition also shows off as a small hump located at $\mathrm{T}_{\mathrm{C}}=674 \pm 10 \mathrm{~K}$. Both values are in good agreement with the specifications provided by the material provider ${ }^{167}$ which are $\mathrm{T}_{\mathrm{X}}^{\text {prov }}=781 \mathrm{~K}$ and $\mathrm{T}_{\mathrm{C}}^{\text {prov }}=672 \mathrm{~K}$.

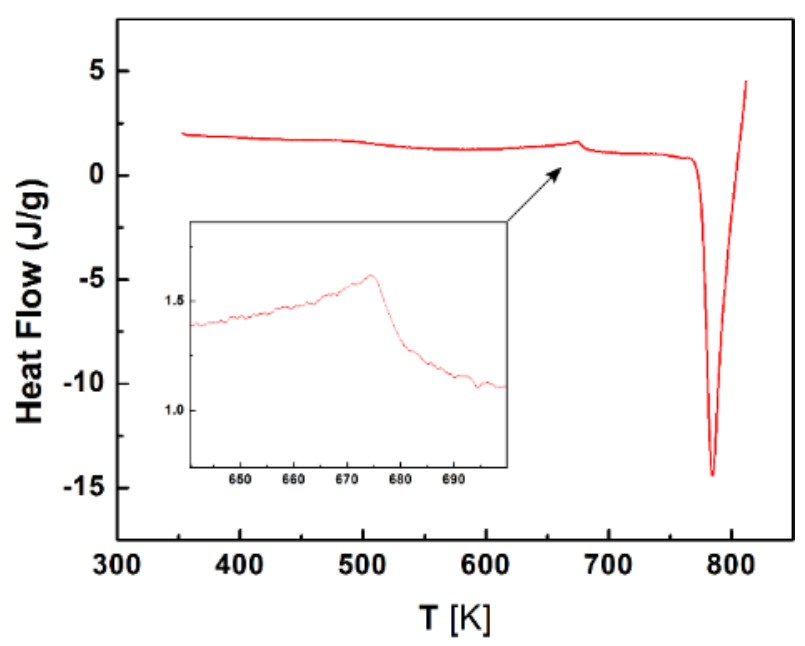

Figure 5.10 DSC scan of $2605 \mathrm{~A} 1$ measured with a heat rate of $\dot{T}=20 \mathrm{~K} / \mathrm{min}$. The Curie and crystallization temperatures can be estimated from the heat hump and crystallization peaks respectively: $\mathrm{T}_{\mathrm{C}}=674 \pm 10 \mathrm{~K}$ and $\mathrm{T}_{\mathrm{X}}=782 \pm 10 \mathrm{~K}$. However the glass transition temperature is difficult to estimate accurately.

In the DSC scan the glass transition is not appreciable. In order to estimate it, temperature scans were performed on $16 \mathrm{~mm} \times 1 \mathrm{~mm} \times 40 \mu \mathrm{m}$ ribbon subjected to different stresses. From the deformation vs Temperature curve, the glass transition can be estimated as the temperature in which the sample increases substantially its flow rate $\mathrm{T}_{\dot{\varepsilon}}$. Figure 5.11 displays the results from the temperature scans and the calculation of $\mathrm{T}_{\dot{\varepsilon}}$ as a function of the applied stress. $\mathrm{T}_{\dot{\varepsilon}}$ measured at the lowest stress is used for the estimation of the glass transition temperature: $\mathrm{T}_{\mathrm{g}} \sim \mathrm{T}_{\dot{\varepsilon}}(\sigma=$ $2.5 \mathrm{MPa})=685 \pm 5 \mathrm{~K}$. 

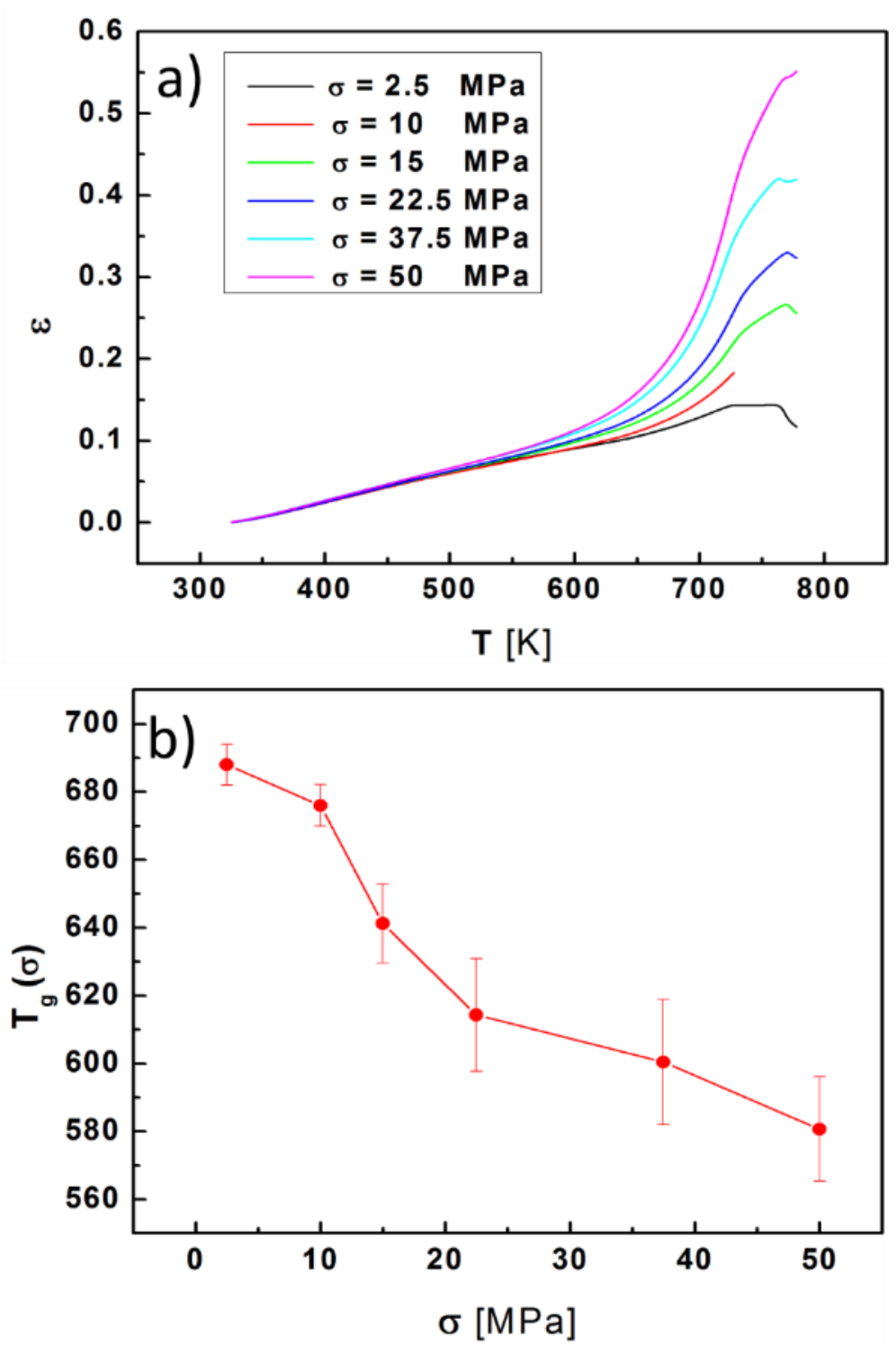

Figure 5.11 (a) Temperature scans of 2605A1 ribbons performed for several constant mechanical loads at a constant heating rate $\dot{\mathrm{T}}=10 \mathrm{~K} / \mathrm{min}$. (b) Temperature in which the strain rate leaves the linear regime $T_{\dot{\varepsilon}}$ as a function of the mechanical load calculated from the curves shown in (a). A linear regression to the data yields a relationship: $T_{g}(\sigma)=(694 \pm 6)-(2.5 \pm 0.3) \sigma$.

The magnetic properties of the glass were characterized measuring the hysteresis loops and the demagnetization curve by means of Vibrating Sample Magnetometer (VSM), as described in section 3. Figure 5.12.a displays the hysteresis loop of a 2605SA1 ribbon. Since the magnetic response depends on the direction of magnetization, the hysteresis loops were measured along the three main axes of symmetry of the ribbon. From the hysteresis loops, the anisotropy fields along the width and length directions can be estimated to be $\mathrm{H}_{\mathrm{A}}^{\mathrm{L}}=110 \pm 20 O e$ and $\mathrm{H}_{\mathrm{A}}^{\mathrm{W}}=300 \pm 200 e$. Along the thickness direction, the magnetization do not increase significantly in this range of fields, as it corresponds to the out-of-plane magnetization of a ribbon shaped sample. The coercive field lays in the interval $\mathrm{H}_{\mathrm{c}}=10 \pm 5 \mathrm{Oe}$ in every orientation. Figure 5.12.b shows the demagnetization curve of the same alloy. The measurement consists in a temperature scan with an applied field of 300 Oe. The Curie temperature corresponds to the temperature at which the sample loses it 
magnetization, and can be estimated from this figure to be $T_{C}=670 \pm 10 \mathrm{~K}$. That value matches the $\mathrm{T}_{\mathrm{C}}$ value obtained from the DSC scan in Figure 5.10. The inset of Figure 5.12.b shows several hysteresis loops measured at different temperatures, and shows how the saturation magnetization decreases with the temperature.
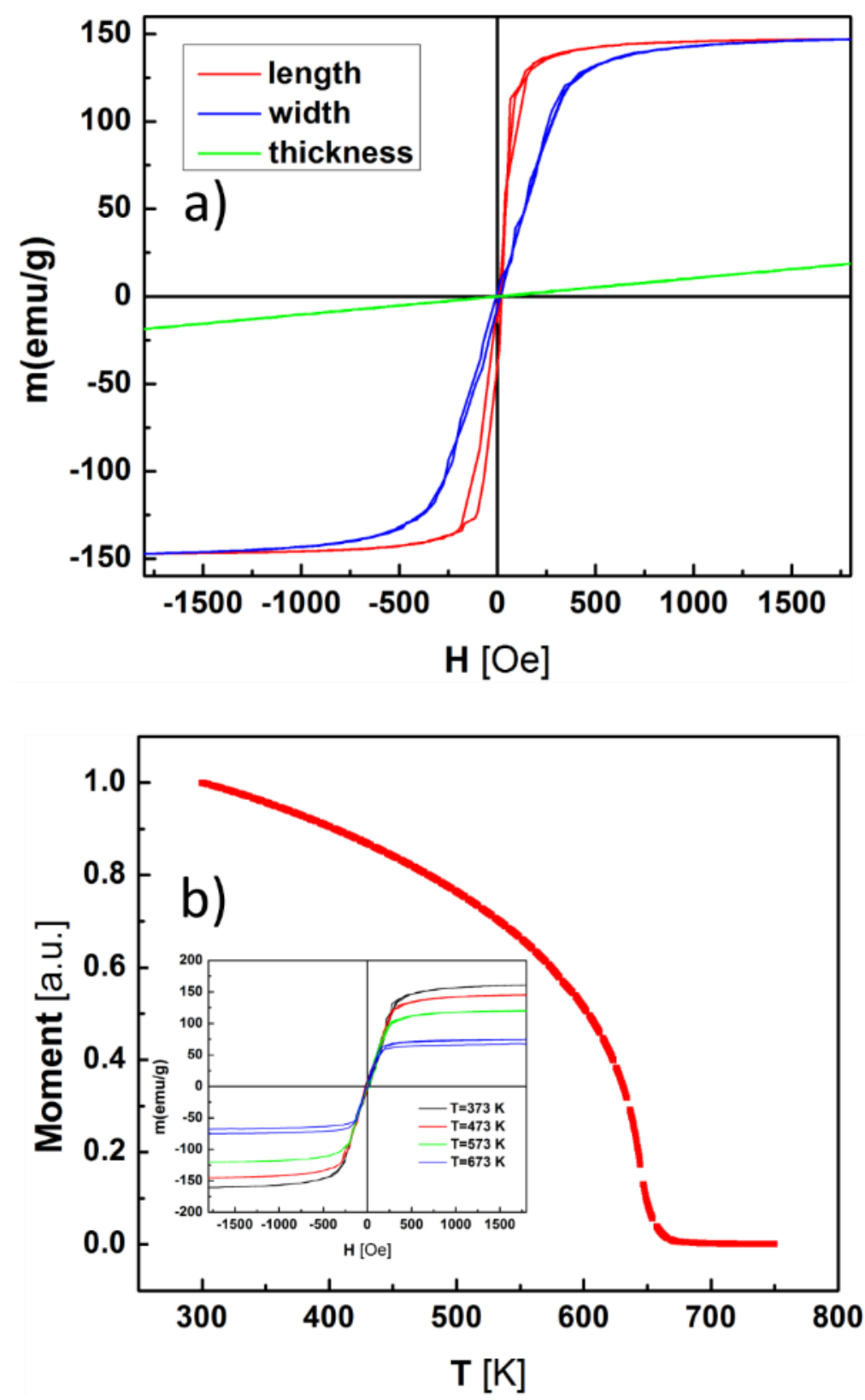

Figure $\mathbf{5 . 1 2}$ (a) Hysteresis loops of 2605SA1 measured by means of VSM. The different curves represent the magnetisation curves along the three main axes of symmetry of the ribbon: length (red), width (blue) and thickness (green). (b) Magnetisation vs Temperature curve M(T) vs T of 2605SA1 measure under a small field $\mathrm{H}=300$ Oe. The inset shows the histeresis curves measured along the width direction at the temperatures: $373,473,573$ and $673 \mathrm{~K}$. 
To check if the samples remained in the amorphous state after the different tests, the structure of each sample was investigated by X-Ray Diffraction experiments. Given the high Fe component of the 2605SA1 alloy, the XRD measurements were performed in the Diffractometer D5000 equipped with a Mo source, as it was discussed in the experimental techniques section.

Figure 5.13 displays several characteristic XRD spectra of the samples investigated through this section. All the spectra show the typical features of an amorphous structure, characterized by the amorphous halo. Also, a second maximum can also be appreciated, which is characteristic of a certain degree of medium range order ${ }^{158}$. The samples kept the amorphous state even under the highest mechanical and magnetic load. The inset of Figure 5.13 shows a fully crystallized 26052A1 ribbon for comparison with the glassy samples. That sample was annealed during $10 \mathrm{~min}$ above the crystallization temperature.

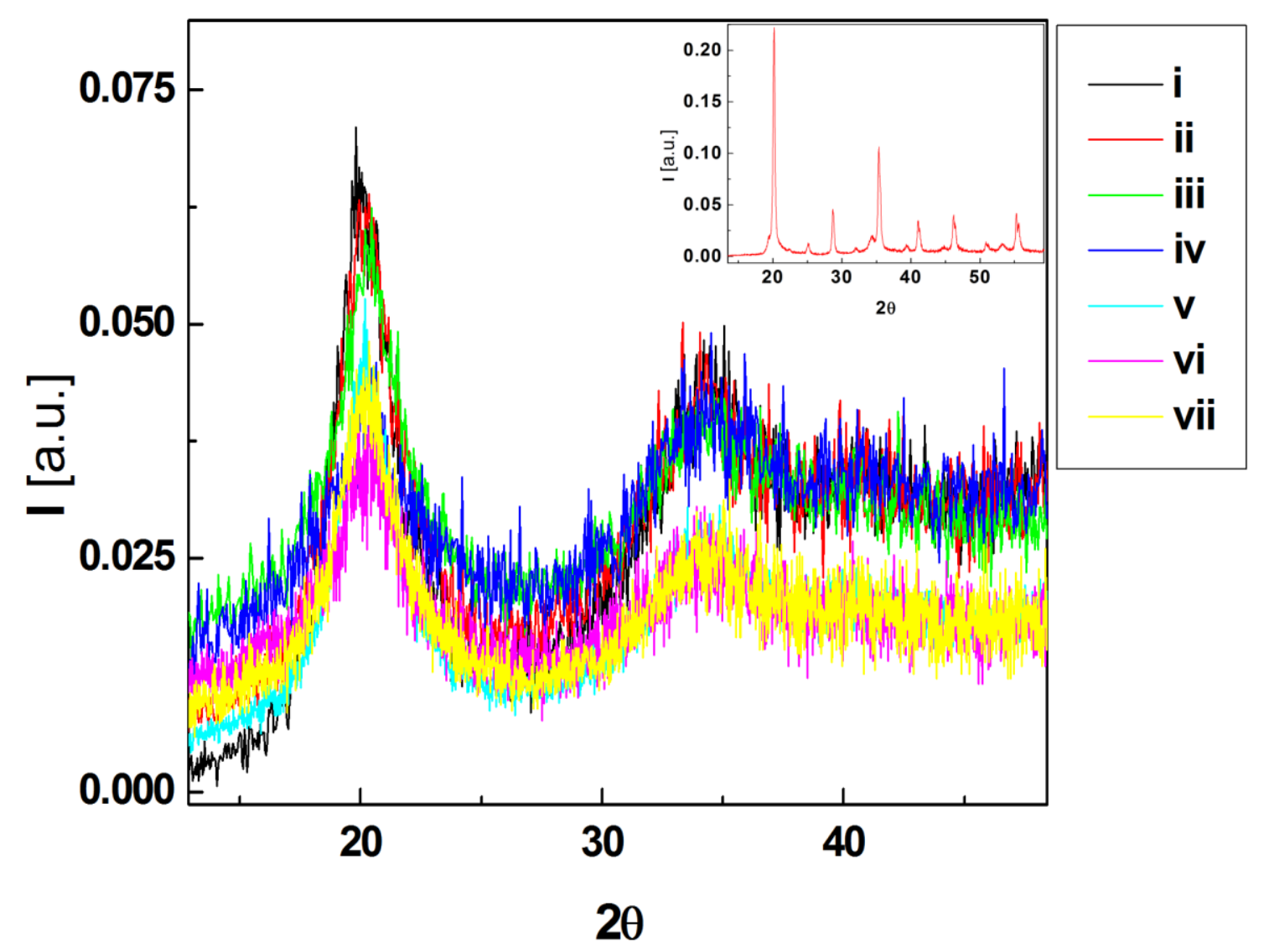

Figure 5.13 XRD spectra of the 2605SA1 alloy. i) As cast. ii) $\frac{\mathrm{T}}{\mathrm{T}_{\mathrm{g}}}=0.8, \sigma=15 \mathrm{MPa}$. iii) $\frac{\mathrm{T}}{\mathrm{T}_{\mathrm{g}}}=0.95, \sigma=15 \mathrm{MPa}$. iv) $\frac{\mathrm{T}}{\mathrm{T}_{\mathrm{g}}}=0.95, \sigma=15 \mathrm{MPa}$. v) $\frac{\mathrm{T}}{\mathrm{T}_{\mathrm{g}}}=0.8, \sigma=15 \mathrm{MPa}$, $|\vec{H}|=500, \theta=0, \phi=0$. vi) $\frac{\mathrm{T}}{\mathrm{T}_{\mathrm{g}}}=0.8, \sigma=15 \mathrm{MPa},|\vec{H}|=200, \theta=0, \phi=45$. Inset: Fully crystallized sample annealed 10 minutes above $\mathrm{T}_{\mathrm{X}}$ 


\subsubsection{The influence of temperature on creep deformation.}

The current subsection focuses on the study of the role of the temperature on the creep deformation of the 2605SA1 glass. In order to do that, several experiments were carried out sweeping the temperature in the range $\frac{\mathrm{T}}{\mathrm{T}_{\mathrm{g}}}=0.7-1$. All tests were done in absence of magnetic field and with the same constant mechanical stress $\sigma=15 \mathrm{MPa}$, for a duration of two hours. Before starting the creep tests, the samples were equilibrated at the working temperature during 5 minutes subjected to a small stress $\sigma=2 \mathrm{MPa}$.

Figure 5.14 shows the creep curves for the different experimental conditions. From the inspection of Figure 5.14(a) it can be seen that the strain exerted by the sample increases with the working temperature. In addition, the curvature of the creep curves seems also to be influenced by the temperature, since the average slope increases as the temperature is increased.

Figure 5.14(b) displays the waiting time distribution corresponding to the creep curves shown in Figure 5.14(a). A clear power law regime can be seen for each of the experiments, followed by a cut-off whose value decreases with increasing temperature. Also, the distributions show an initial higher slope which is characteristic of the crossover. In the case of the experiment performed at $\frac{\mathrm{T}}{\mathrm{T}_{\mathrm{g}}}=0.7$ the sample did not show enough deformation in order to measure the waiting times. Therefore, it was not consider for further analysis. 

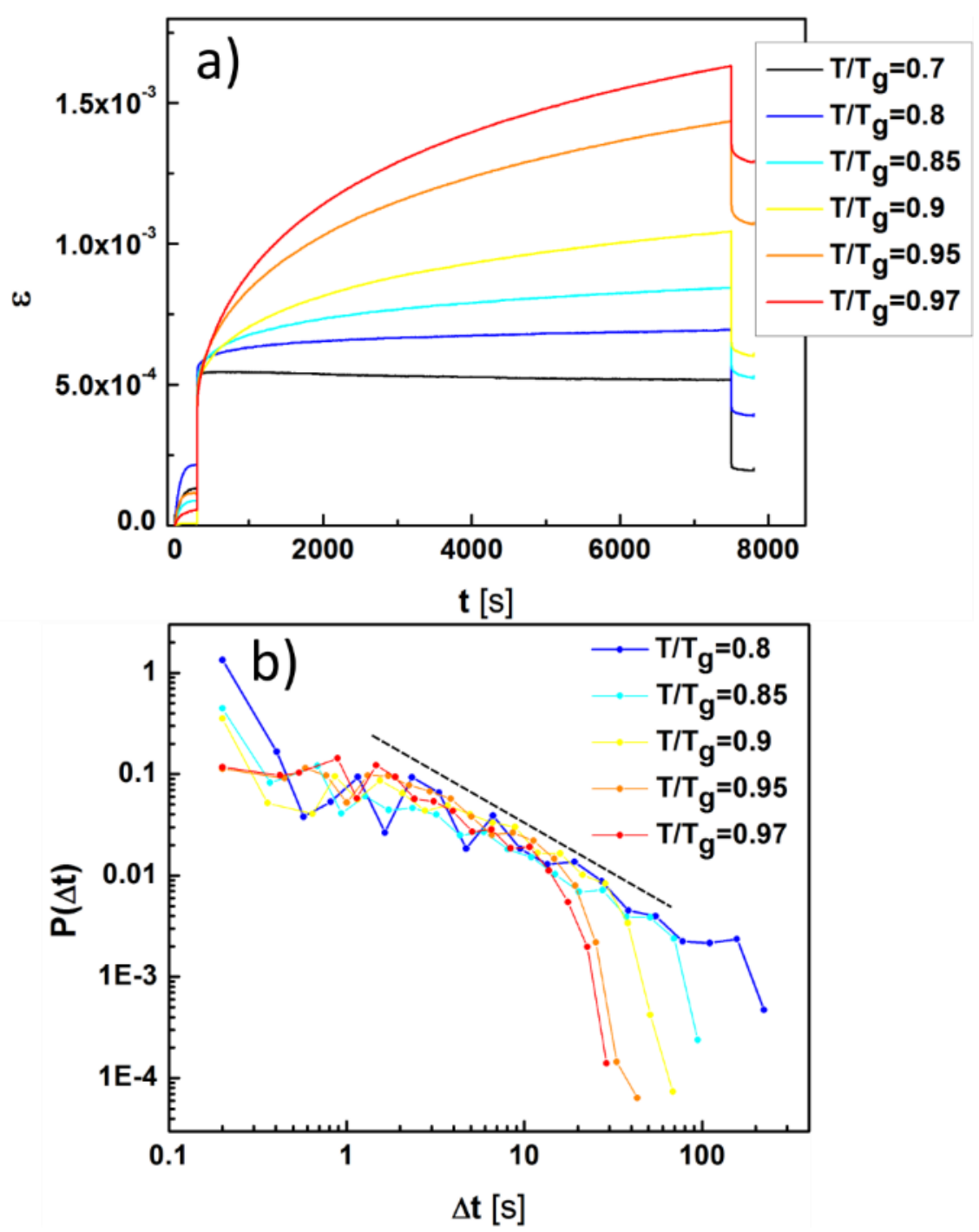

Figure 5.14 (a) Creep tests of 2605SA1 ribbons, all subjected to the same mechanical stress ( $\sigma=15 \mathrm{MPa}$ ) and different temperatures. (b) Waiting time distribution calculated from the creep curves shown in (a). The dashed line represents a perfect power law with exponent $\tau=-0.8$.

Figure 5.15 depicts the waiting time distribution before and after the crossover time $t_{\text {cross }}$ for each of the creep measurements shown in Figure 5.14 . In each of the experiments, the waiting time distribution shows a power law shape. In every case, the waiting time distribution changes from a steeper exponent at lower experimental times to a less step distribution at longer waiting times. Figure 5.15. (f) shows the numerical fit of the power law exponents before and after the crossover. It shows that most of the exponents of the power law distribution before the crossover $\left(\tau_{1}\right)$ lay within the range $\tau_{1}=-1.5 \pm 0.1$. Additionally, the exponents of the waiting time distribution after the crossover $\left(\tau_{2}\right)$ show values in the range $\tau_{2}=-0.8 \pm 0.1$, 

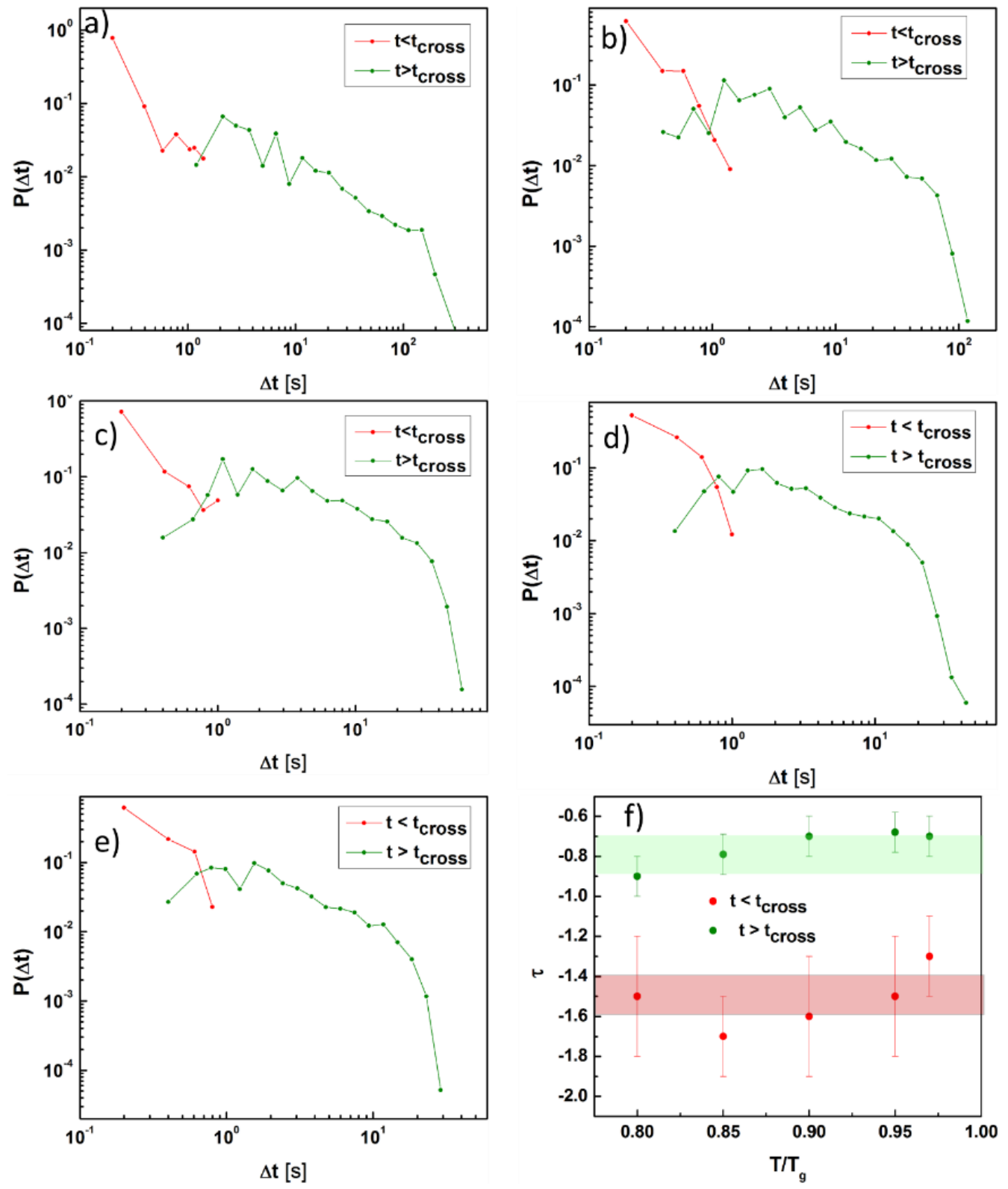

Figure 5.15 Waiting time distributions before and after the crossover time, for the creep measurements on Fe -rich 2605SA1 under several temperatures. a) $\frac{\mathrm{T}}{\mathrm{T}_{\mathrm{g}}}=$ $0.8, \sigma=15 \mathrm{MPa}$. (b) $\frac{\mathrm{T}}{\mathrm{T}_{\mathrm{g}}}=0.85, \sigma=15 \mathrm{MPa}$. (c) $\frac{\mathrm{T}}{\mathrm{T}_{\mathrm{g}}}=0.9, \sigma=15 \mathrm{MPa}$. (d) $\frac{\mathrm{T}}{\mathrm{T}_{\mathrm{g}}}=$ $0.95, \sigma=15 \mathrm{MPa}$. (e) $\frac{\mathrm{T}}{\mathrm{T}_{\mathrm{g}}}=0.97, \sigma=15 \mathrm{MPa}$. (f) Fit of the power law exponents before and after the crossover calculated from the curves (a)-(e), the dashed lines represent the intervals $\tau_{1}=-1.5 \pm 0.1$ and $\tau_{2}=-0.8 \pm 0.1$.

Figure 5.16 shows the dependence of $t_{\text {cross }}$ and $t_{\dot{\varepsilon}}$ with the temperature, calculated following the procedure indicated in section 4 . Both quantities $t_{\text {cross }}$ and $t_{\dot{\varepsilon}}$ seem to increase with the temperature, although $t_{\text {cross }}$ appears to be less temperature sensitive as it was in the case of a $\mathrm{Pd}_{77.5} \mathrm{Cu}_{6} \mathrm{Si}_{16.5}$. A physical interpretation for this difference is given in Chapter 6 . 


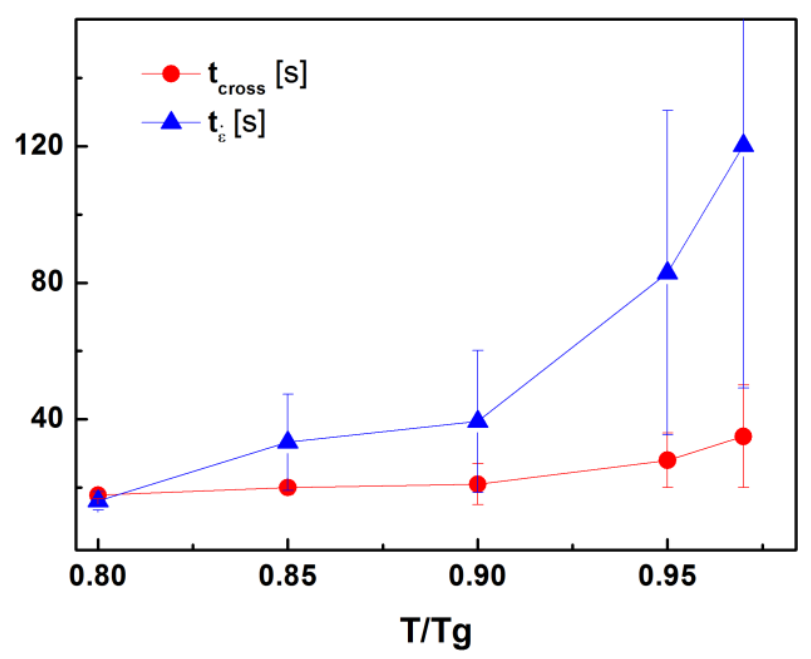

Figure 5.16 Dependence of $t_{\text {cross }}$ and $t_{\dot{\varepsilon}}$ with the temperature at a fixed stress $\sigma=$ $15 \mathrm{MPa}$.

The creep measurements were also analyzed from the point of view of the dispersion of the energy barriers, making use of the figure of merit $W$ defined in in Chapter 4. Figure 5.17(a) shows the time evolution of the effective energy barriers calculated making use of the transition state theory for each of the working temperature. Figure 5.17 (b) depicts the evolution of the residuals, which are the difference between the experimental Energy values and a non-parametric LOESS fit to each of the curves depicted in Figure 5.17 (a). The probability distribution of the residuals of the energy barrier is shown in Figure 5.17(c). From Figure 5.17(b) and (c) it can be seen that the dispersion increases with the temperature. The amount of the dispersion is quantified by $W$, defined as the Full Width Half Maximum FWHM of the probability distributions shown in Figure 5.17 (c). The dependence of $W$ with the temperature is shown in Figure 5.17(d) 

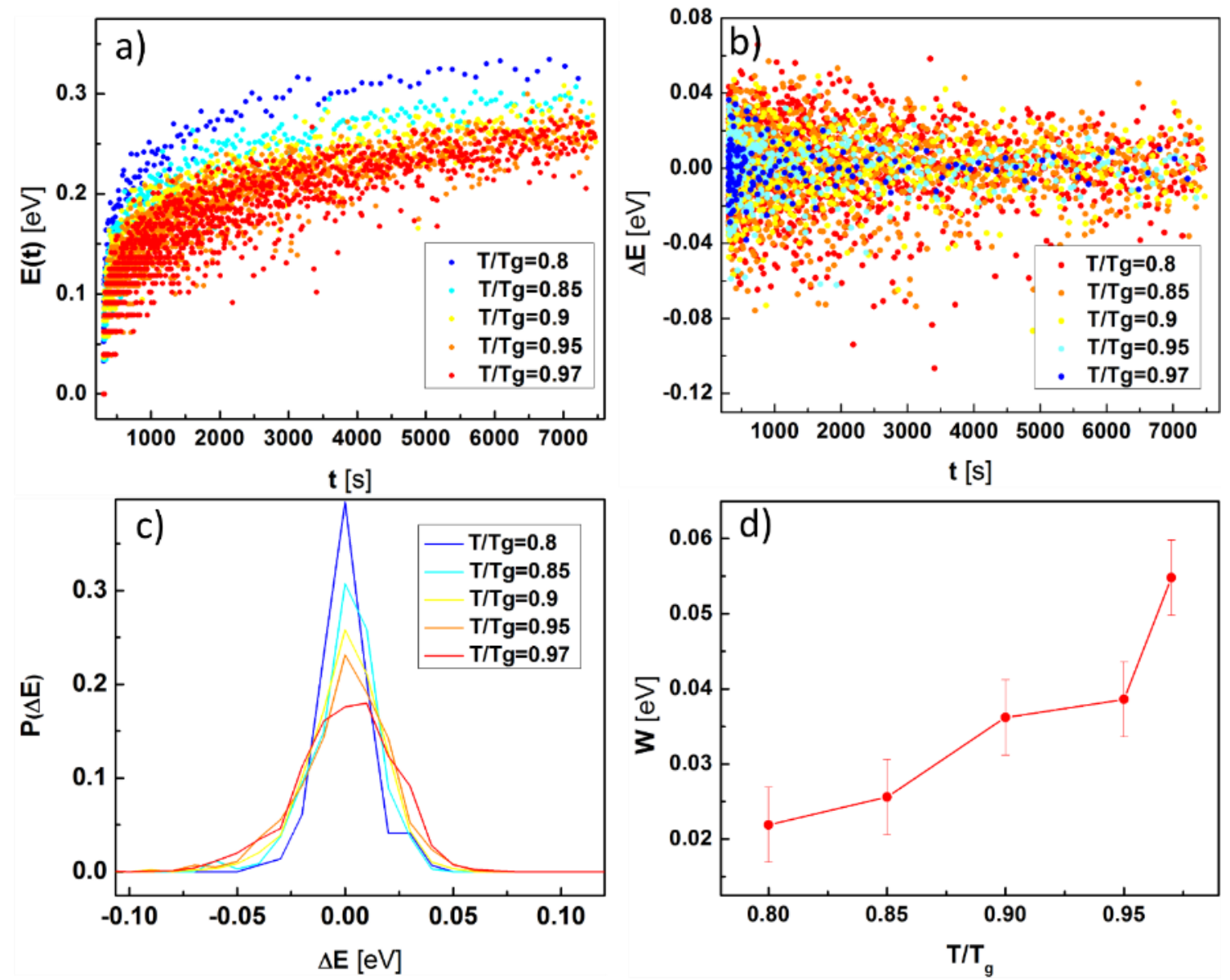

Figure 5.17 (a) Effective energy barrier as a function of experimental time calculated from the Transition State Theory for the creep measurements done at $\sigma=15 \mathrm{MPa}$ and several temperatures. (b) Residuals Vs time. The residuals are calculated as the difference between the energy values and a LOESS fit. (c) Probability distribution of the residuals for the different temperatures. (d) Evolution of $W$ as a function of temperature.

Figure 5.17 (d) reveals that $W$ shows a similar temperature dependence as $t_{\text {cross }}$ and $t_{\dot{\varepsilon}}$. The connection between the three figures of merit will be further discussed in Chapter 6 . 


\subsubsection{The influence of mechanical stress on creep deformation}

In this subsection the influence of the mechanical stress on the creep measurements of 2605SA1 is analyzed. In order to do that, the temperature was kept constant to $\frac{\mathrm{T}}{\mathrm{T}_{\mathrm{g}}}=0.8$ and several creep experiments were performed, sweeping the mechanical stress in the range $\sigma=11-50 \mathrm{MPa}$. The upper stress limit is determined by the maximum force that can be applied by the DMA, whereas the minimum is determined by the minimum stress that produces appreciable deformation on the samples. Figure 5.18(a) depicts the creep curves measured at different mechanical stresses. The average slope of the curves increases with the stress and so does in general the initial instantaneous elastic deformation. Only in the case of $\sigma=50$ and $\sigma=25$ this order is inverted. That may be due to differences in the clamping, but it does not affect the waiting time analysis since the elastic deformation is not taken into account for it.

Figure 5.18(b) shows the waiting time distribution corresponding to the creep measurements presented in Figure 5.18(a). A power law regime over almost three decades can be observed, and the dashed line suggests a similar slope for each of the cases. A crossover on the scaling can also be appreciated, since the distribution of the short waiting times present a steeper slope than the long ones. The mechanical stress affects as well the cut-off, which shift to lower waiting times as the stress increases. 

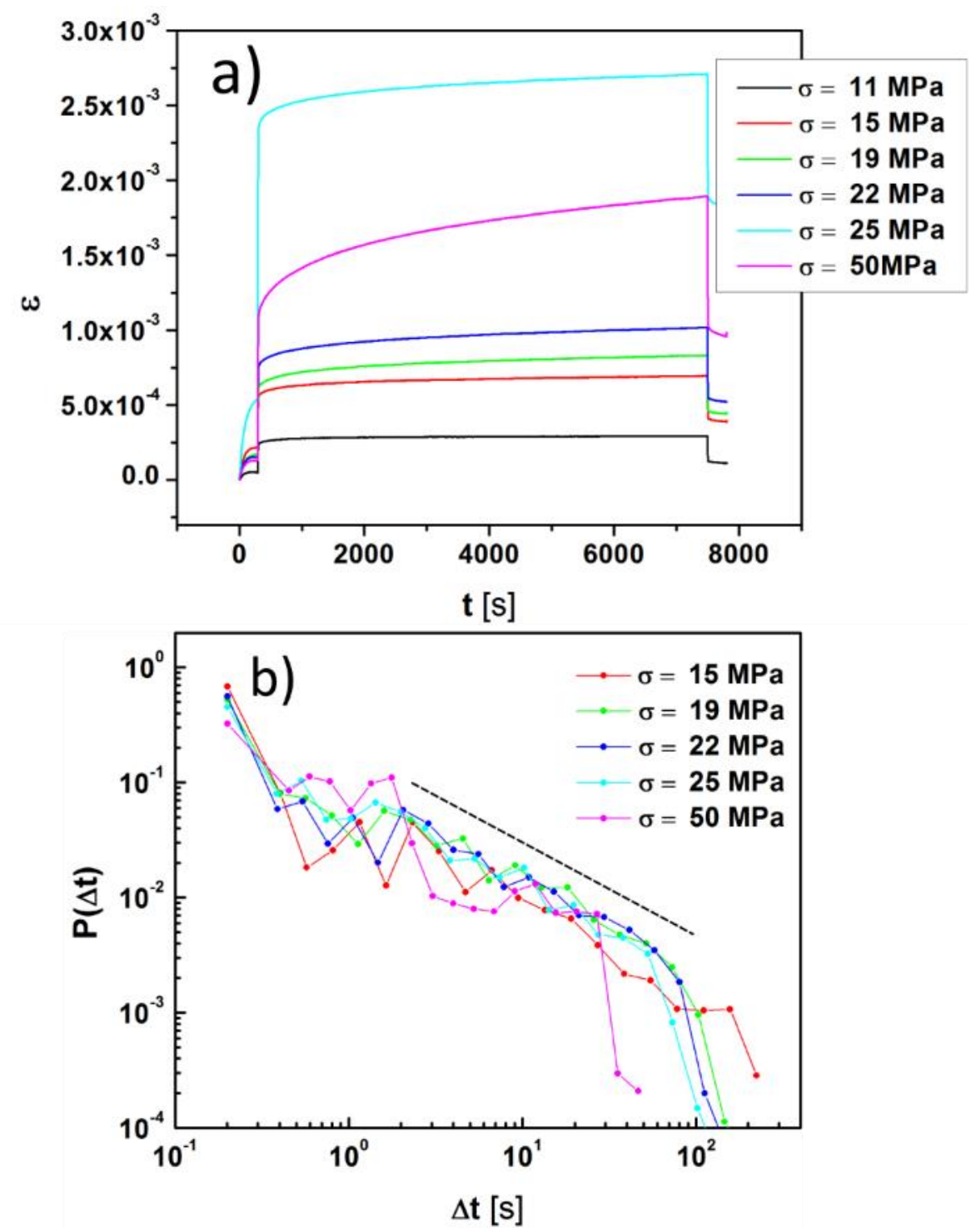

Figure 5.18 (a) Creep tests of 2605SA1 ribbons subjected to different mechanical stresses and kept at the same temperature $\frac{\mathrm{T}}{\mathrm{T}_{\mathrm{g}}}=0.8$. (b) Waiting time distribution calculated from the creep curves shown in (a). The dashed line represents a perfect power law with exponent $\tau=-0.8$.

Figure 5.19 displays the distribution of waiting times separated at the crossover time $t_{\text {cross }}$. The waiting time distribution from each of the experiments presents a clear power law regime except the case of the highest stress $\sigma=50 \mathrm{MPa}$. In that case the distribution of the waiting times for $t>$ $t_{\text {cross }}$ is not a straight line anymore, but shows a hump at long waiting times, a feature that will be discussed in the next chapter. Finally, Figure 5.18(f) show numerical fits of the power laws exponents before $\left(\tau_{1}\right)$ and after $\left(\tau_{2}\right)$ the crossover for each of the experiments. It shows that both exponents take the values $\tau_{1}=-1.5 \pm 0.1$ and $\tau_{2}=-0.8 \pm 0.1$. 

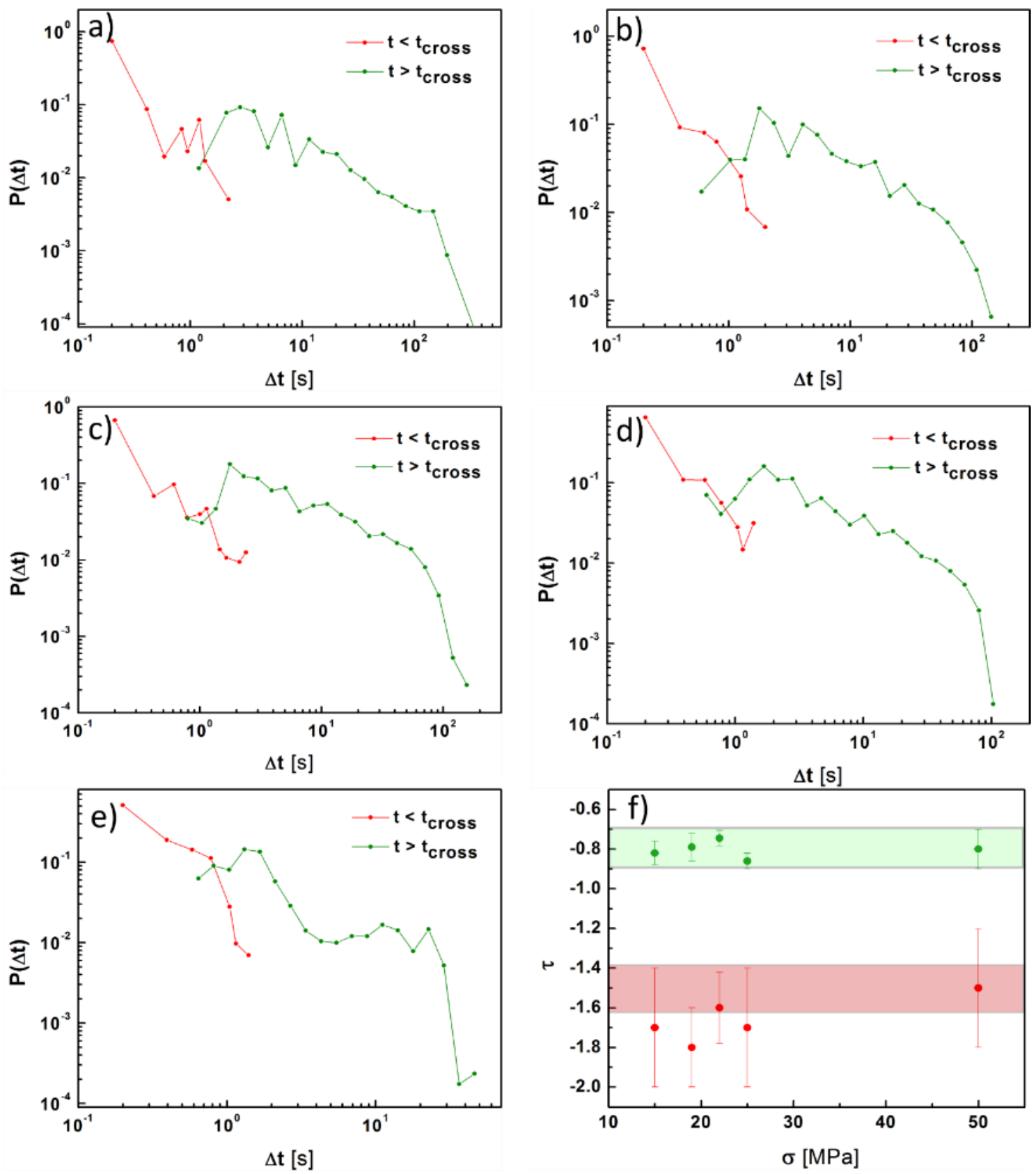

Figure 5.19 Waiting time distributions before and after the crossover time, for the creep measurements of 2605SA1 ribbons measured at temperature $\frac{\mathrm{T}}{\mathrm{T}_{\mathrm{g}}}=0.8$ and under several mechanical stresses. (a) $\sigma=15 \mathrm{MPa}$. (b) $\sigma=19 \mathrm{MPa}$. (c) $\sigma=$ $22 \mathrm{MPa}$. (d) $\sigma=25 \mathrm{MPa}$. (e) $\sigma=50 \mathrm{MPa}$. (f) Fit of the power law exponents before and after the crossover calculated from the curves (a)-(e), the dashed lines represent the intervals $\tau_{1}=-1.5 \pm 0.1$ and $\tau_{2}=-0.8 \pm 0.1$.

The creep measurements are also analyzed from the point of view of the dispersion of the energy barriers. Following a similar procedure as it was shown in Figure 5.17, $W$ can be calculated from each creep curve, providing a quantitative measure of the dispersion. Figure 5.20 shows the dependence of the three figures of merit $t_{\text {cross }}, t_{\dot{\varepsilon}}$ and $W$ with the mechanical stress. Figure 5.20 (a) depicts the dependence of $t_{\text {cross }}, t_{\dot{\varepsilon}}$ with the applied stress. It can be seen that two magnitudes seem to have a linear dependence with the stress, and their values almost overlap. That's is the 
same case as it was for the $\mathrm{Pd}_{77.5} \mathrm{Cu}_{6} \mathrm{Si}_{16.5}$ measurements. Figure 5.20 (b) shows the functional dependence of $W$ with the mechanical stress, and also a roughly linear dependence can be observed. All three figures of merit show a similar functional dependence with the mechanical stress, a fact that suggests a correlation between the avalanche regime and the dispersion of the energy barriers in the PEL.
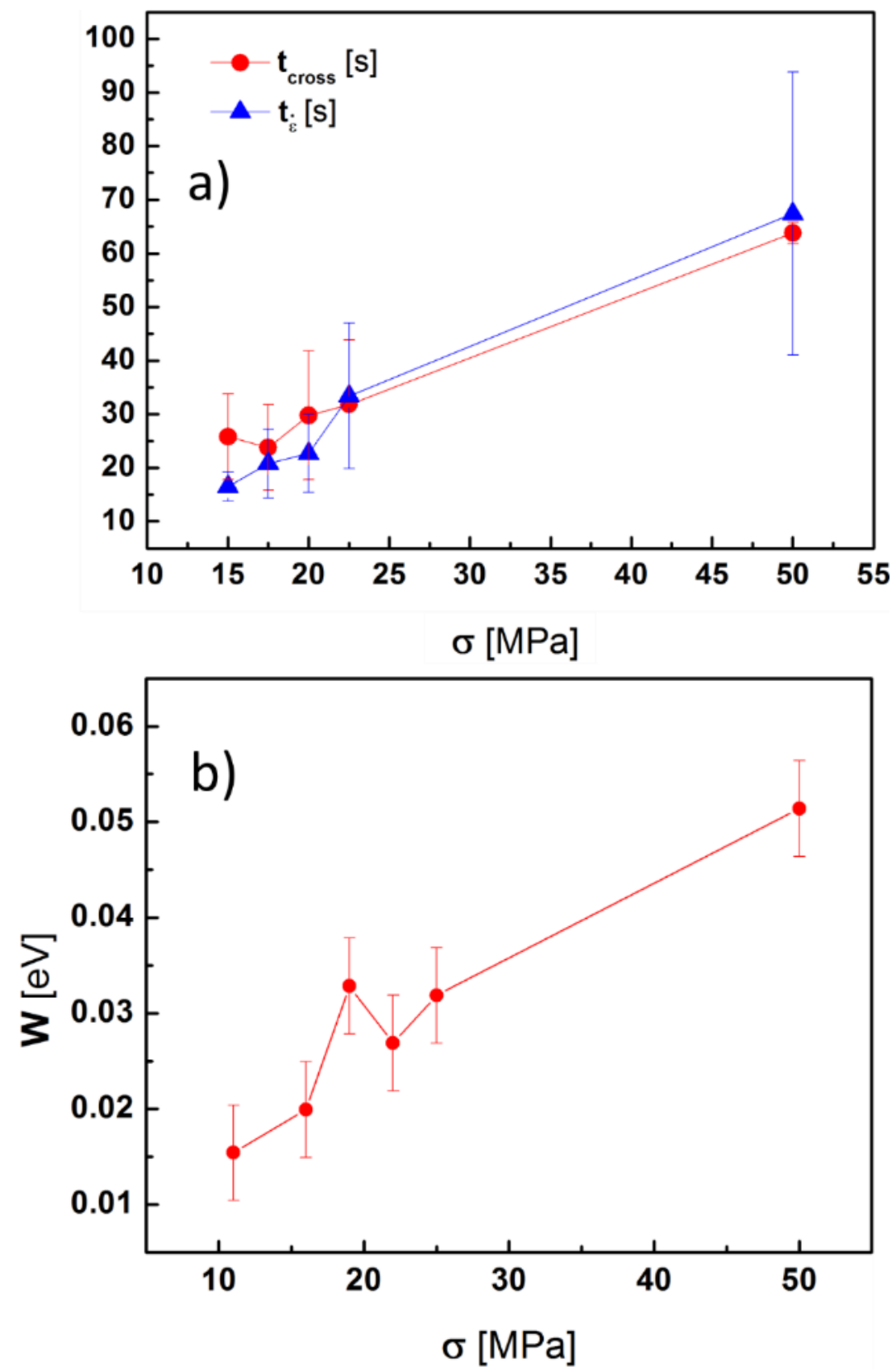

Figure 5.20 (a) Dependence of $t_{\text {cross }}$ and $t_{\dot{\varepsilon}}$ with stress for a fixed temperature $\frac{\mathrm{T}}{\mathrm{T}_{\mathrm{g}}}=0.8$ for the 2605SA1 alloy (b) $W$ as a function of the mechanical stress for the same composition at the same temperature. 


\subsubsection{The influence of magnetic field intensity on creep deformation}

In this subsection and the two following ones the results regarding the study of the influence of the magnetic field on the creep deformation process of 2605SA1 metallic glass are presented. Since the magnetic field is a vectorial quantity, and so is the stress, not only it intensity should be considered but also it orientation with respect to the mechanical stress. Taking that into account, the study of the magnetic field effect is divided in three subsections, which focuses on the influence of it intensity, and orientation. The study of the orientation is as well divided into two independent parts which study the influence of the angle $\theta$ within a plane perpendicular to the stress and the angle $\phi$ with respect to the stress.

The experiments shown in the current subsection focus on the study of the influence of the intensity of the magnetic field. Since it turned out that the influence of the magnetic field depends itself on the mechanical load, all the experiments were performed for two different tensile stresses, $\sigma=15$ and $\sigma=25 \mathrm{MPa}$. Figure 5.21 (a) shows a set of creep experiments performed at $\frac{\mathrm{T}}{\mathrm{T}_{\mathrm{g}}}=0.8$, $\sigma=15$ and several intensities of magnetic field applied along the width direction of the ribbon $(\theta=0, \phi=0)$. As shown in Figure 5.12 the sample magnetization decreases with temperature. Therefore, the temperature of the experiments was chosen to be the lowest at which the sample deforms sufficiently in order to be able to perform a waiting times analysis.

Figure 5.21(b) shows the waiting time distribution calculated for the experiments shown in Figure 5.21(a). A power law regime can be appreciated in the double logarithmic plot, as well as an initial higher slope, which suggest a crossover in the waiting time distribution. The cut-off is shifted to shorter waiting times when the magnetic field was applied. 

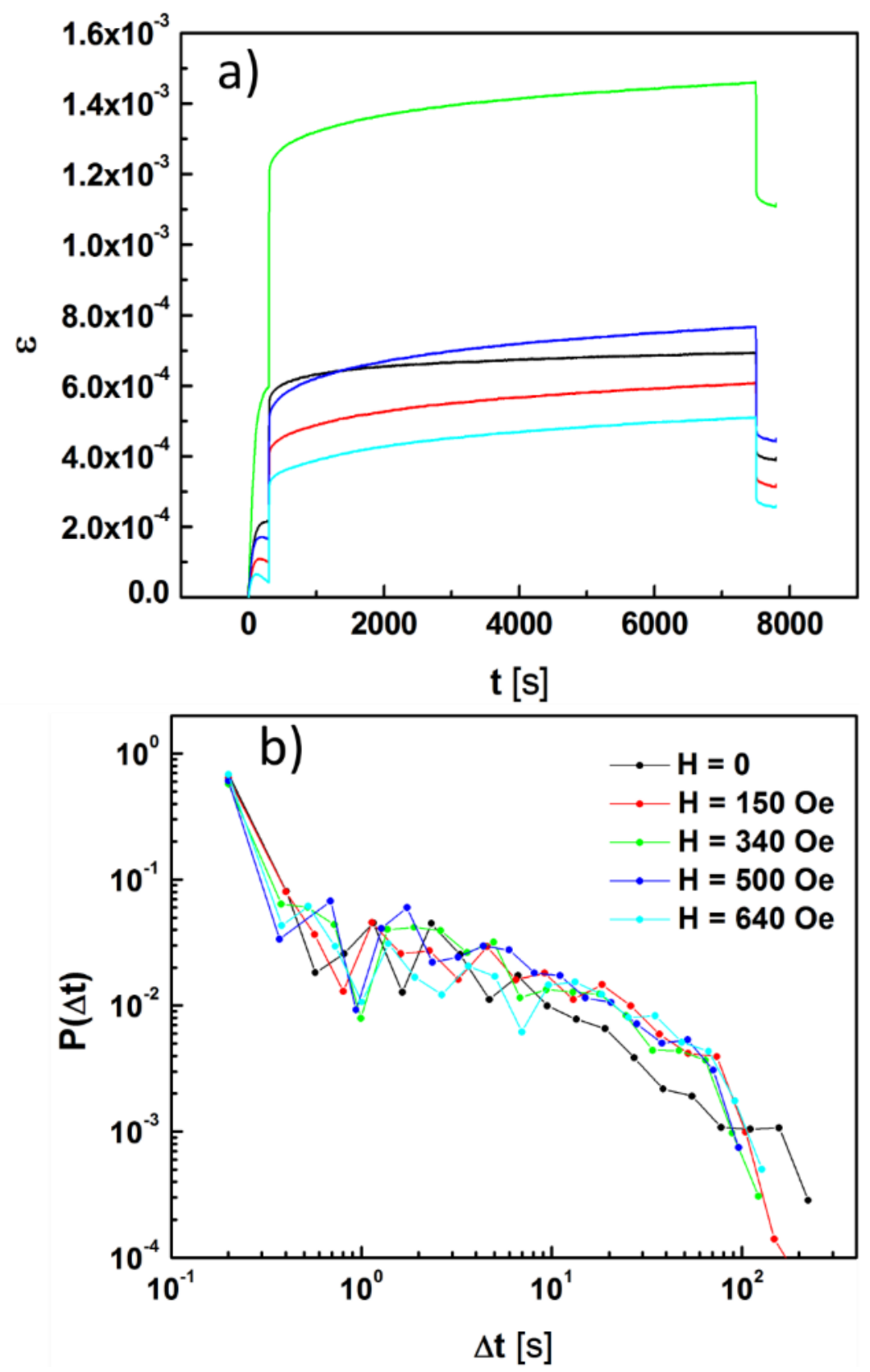

Figure 5.21 (a) Creep measurements of 2605SA1 performed at $\frac{T}{T_{g}}=0.8, \sigma=15$ for different intensities of magnetic field oriented along the width direction $(\theta=0, \phi=0)$. (b) Double logarithmic representation of the waiting time distribution calculated from the creep measurements shown in (a)

Figure 5.22 shows the waiting time distribution splitting the data between before and after the crossover time $t_{\text {cross }}$. It can be seen in Figure 5.22 (a)-(e) that there is a significant change of slope at $t_{\text {cross }}$, but the power law shape is well preserved in each of the cases. Figure 5.22 (f) shows the fit of the experimental power laws and it demonstrates that, except the measurement at $|\vec{H}|=$ $640 \mathrm{Oe}$, the values of the experimental exponents before $\left(\tau_{1}\right)$ and after $\left(\tau_{2}\right)$ the crossover are contained in the range $\tau_{1}=-1.5 \pm 0.1$ and $\tau_{2}=-0.8 \pm 0.1$. The experiment corresponding to $|\vec{H}|=640$ Oe presents a lower slope of $\tau_{2} \sim-0.5$ in the second regime. 

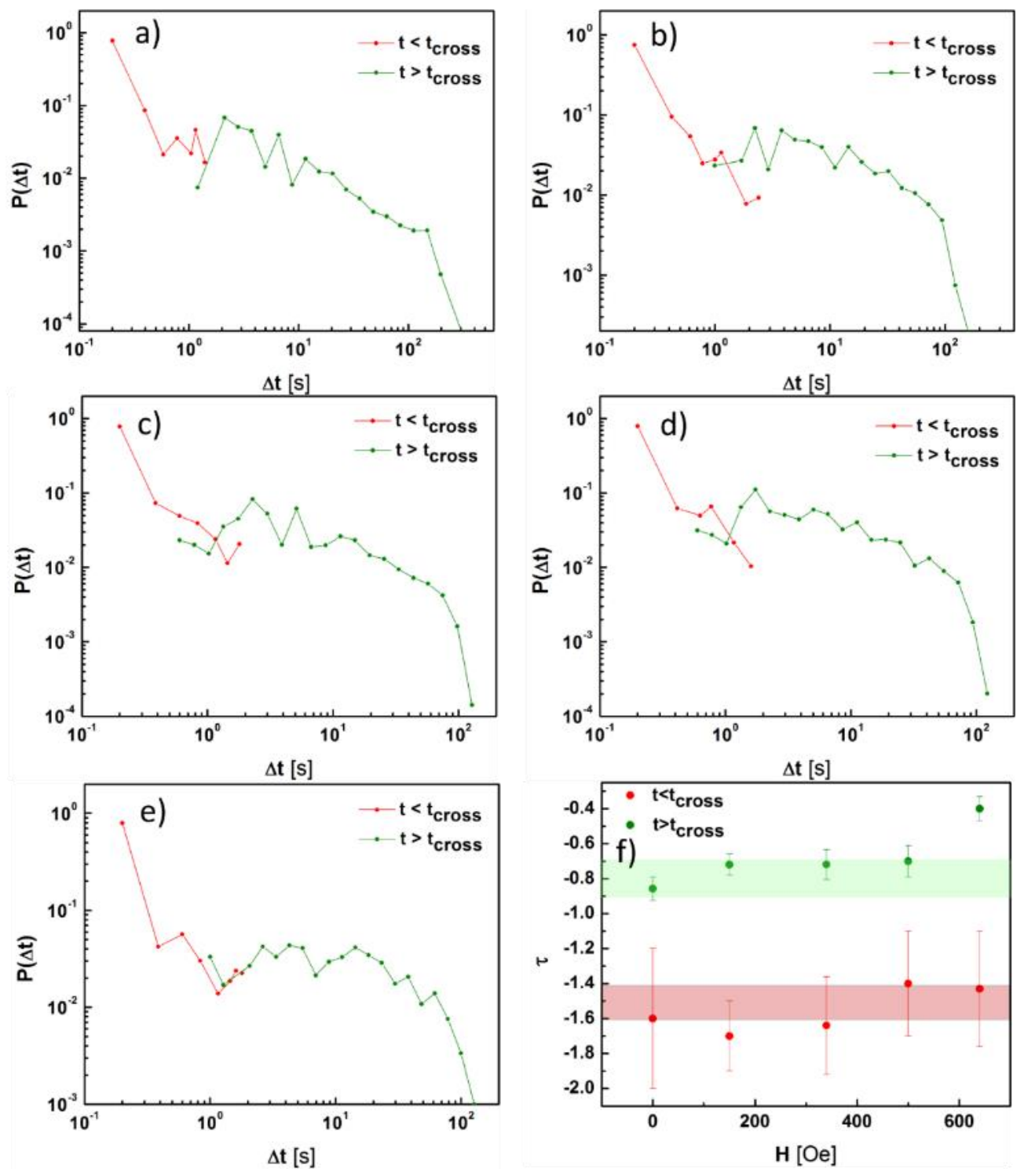

Figure 5.22 Waiting time distributions before and after the crossover time, for the creep measurements of 2605SA1 ribbons at temperature $\frac{T}{T_{g}}=0.8, \sigma=15 \mathrm{MPa}$ and under several intensities of magnetic field along the width direction $(\theta=0, \phi=0)$. (a) $|\vec{H}|=0$ Oe. (b) $|\vec{H}|=150$ Oe. (c) $|\vec{H}|=340$ Oe. (d) $|\vec{H}|=500$ Oe. (e) $|\vec{H}|=640$ Oe. (f) Fit of the power law exponents before and after the crossover calculated from the curves (a)-(e), the shaded areas represent the intervals $\tau_{1}=-1.5 \pm 0.1$ and $\tau_{2}=-0.8 \pm 0.1$.

The three figures of merit, $t_{\text {cross }}, t_{\dot{\varepsilon}}$ and $W$, are shown in Figure 5.23. All of three magnitudes present a maximum which is located in the range 150-500 Oe, which matches the value of the anisotropy field along the width orientation $\mathrm{H}_{\mathrm{A}}^{\mathrm{W}}=300 \pm 20$ 0e shown in figure 5.12. All the values of applied field are higher than the coercive field which is estimated to be $\mathrm{H}_{\mathrm{c}} \approx 10 \mathrm{Oe}$. Such maximum of the figures of merit can be interpreted in terms of the orientation of domain walls and and will be discussed in next section. 

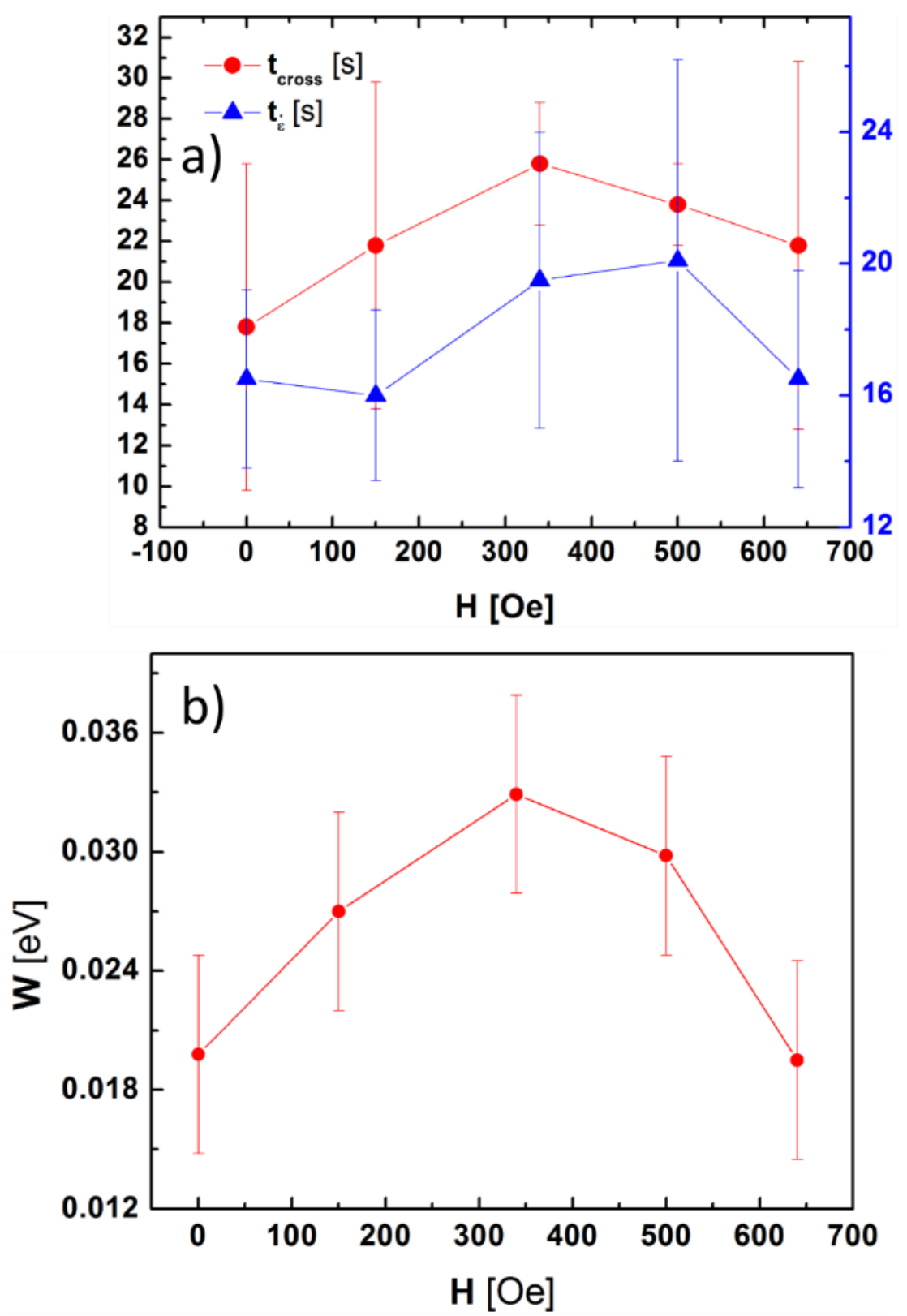

Figure 5.23 (a) Dependence of $t_{\text {cross }}$ and $t_{\dot{\varepsilon}}$ with the magnetic field intensity applied along the width direction $(\theta=0, \phi=0)$, calculated from creep measurements on 2605SA1 ribbons for a fixed temperature $\frac{T}{T_{g}}=0.8$ and several stresses. (b) $W$ as a function of magnetic field intensity, applied along the width direction $(\theta=0, \phi=0)$, calculated from creep measurements on 2605SA1 ribbons for a fixed temperature $\frac{T}{T_{g}}=0.8$ and stress $\sigma=15 \mathrm{MPa}$.

As was previously stated, the influence of the magnetic field intensity is expected to be stress dependent. All the measurements shown in this subsection, were repeated for a higher value of mechanical stress $\sigma=25 \mathrm{MPa}$. Figure 5.24 (a) displays the creep curves measured at $\frac{\mathrm{T}}{\mathrm{T}_{\mathrm{g}}}=0.8, \sigma=$ 25 and several intensities of magnetic field applied along the width direction of the ribbon $(\theta=0, \phi=0)$. Figure 5.24(b) shows the waiting time distribution calculated from such creep measurements. A power law regime can be clearly seen, as well as an initial higher slope, which suggest a crossover in the waiting times statistics. 

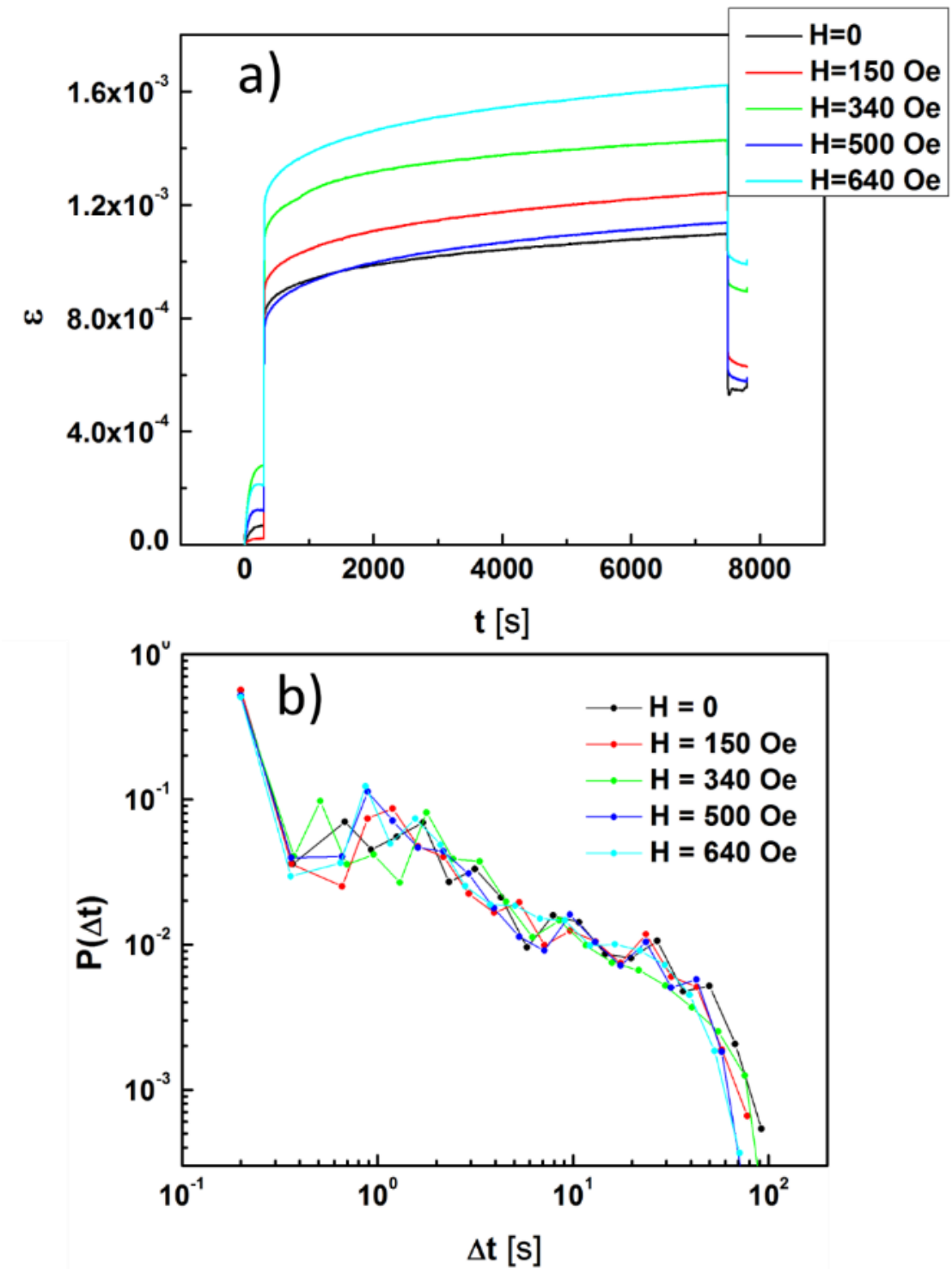

Figure 5.24 (a) Creep measurements of 2605SA1 perfermed at $\frac{T}{T_{g}}=0.8, \sigma=25$ for different intensities of magnetic field oriented along the width direction $(\theta=0, \phi=0)$. (b) Double logarithmic representation of the waiting time distribution calculated from the creep measurements shown in (a)

Figure 5.25 shows the waiting time distribution splitting the data between before and after the crossover time $t_{\text {cross }}$. It can be seen in Figure 5.25(a)-(e) that there is a significant change of slope at $t_{\text {cross }}$, but the power law shape is well preserved in each of the cases. Figure 5.22(f) shows the fit of the experimental power laws and it can be seen that the experimental exponents before $\left(\tau_{1}\right)$ and after $\left(\tau_{2}\right)$ the crossover oscillate around the values $\tau_{1}=-1.5$ and $\tau_{2}=-0.8$. However, in this set of experiments some of the deviations are larger than \pm 0.1 . particularly, the experiment performed at $\mathrm{H}=340$ Oe shows a dip and peak of the first and second exponents $\tau_{1}$, $\tau_{2}$ respectively. 

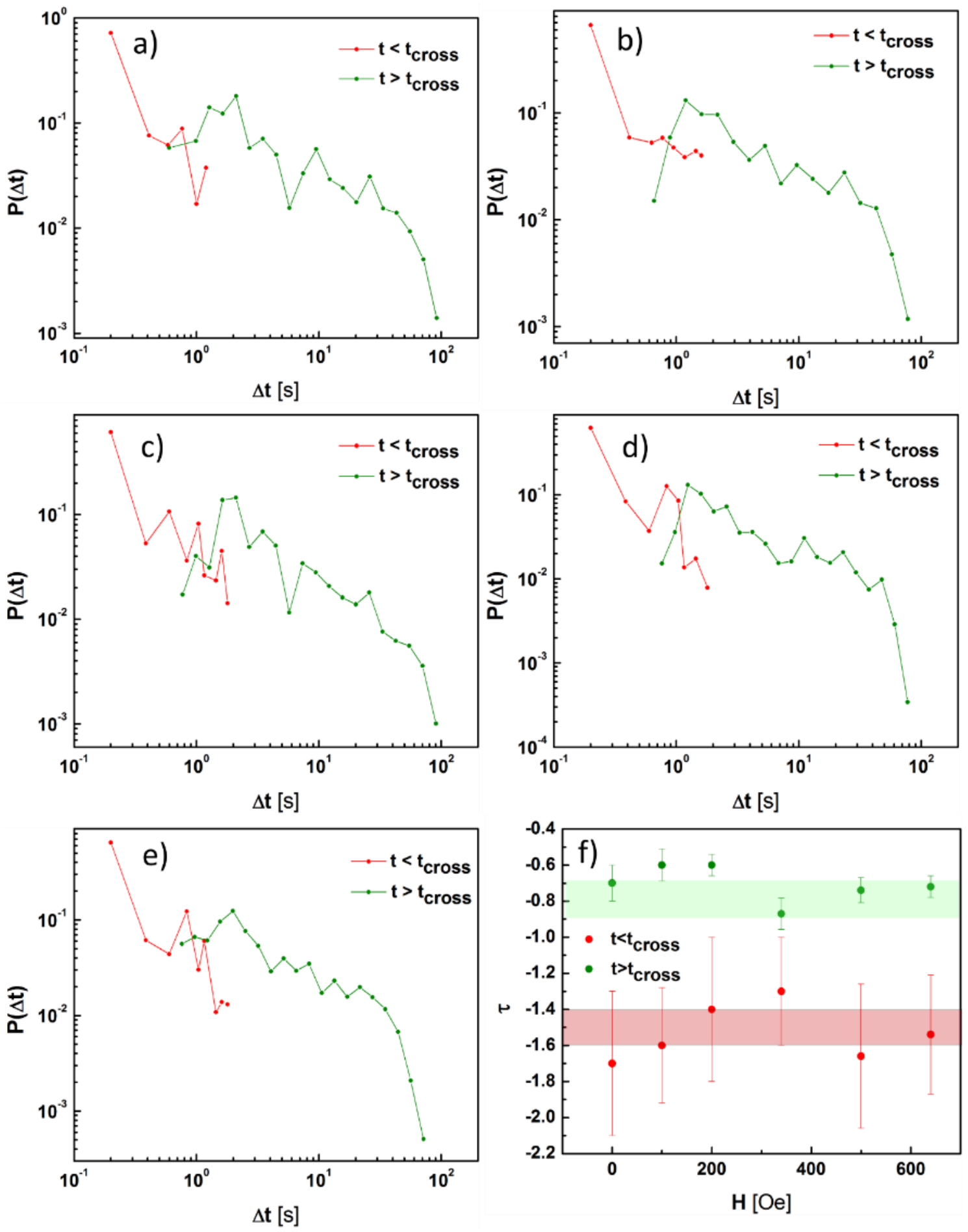

Figure 5.25 Waiting time distributions before and after the crossover time, for the creep measurements of 2605SA 1 ribbons at temperature $\frac{T}{T_{g}}=0.8, \sigma=25 \mathrm{MPa}$ and under several intensities of magnetic field along the width orientation $(\theta=0, \phi=0)$. (a) $|\vec{H}|=0$ Oe. (b) $|\vec{H}|=150$ Oe. (c) $|\vec{H}|=340$ Oe. (d) $|\vec{H}|=500$ Oe. (e) $|\vec{H}|=640$ Oe. (f) Fit of the power law exponents before and after the crossover calculated from the curves (a)-(e), the dashed areas represent the intervals $\tau_{1}=-1.5 \pm 0.1$ and $\tau_{2}=-0.8 \pm 0.1$. 
Last, the three figures of merit, $t_{\text {cross }}, t_{\dot{\varepsilon}}$ and $W$, are shown in Figure 5.26. In contrast with the creep measurements at $\sigma=15 \mathrm{MPa}$, these magnitudes do not show a maximum. Instead, they increase almost monotonically with increasing magnetic field. This change in behavior in comparison with the measurements at $\sigma=15 \mathrm{MPa}$ will be analyzed and discussed in Section 6 , and will be interpreted in terms of the anisotropy field $H_{A}(\sigma)$, which is stress-dependent.
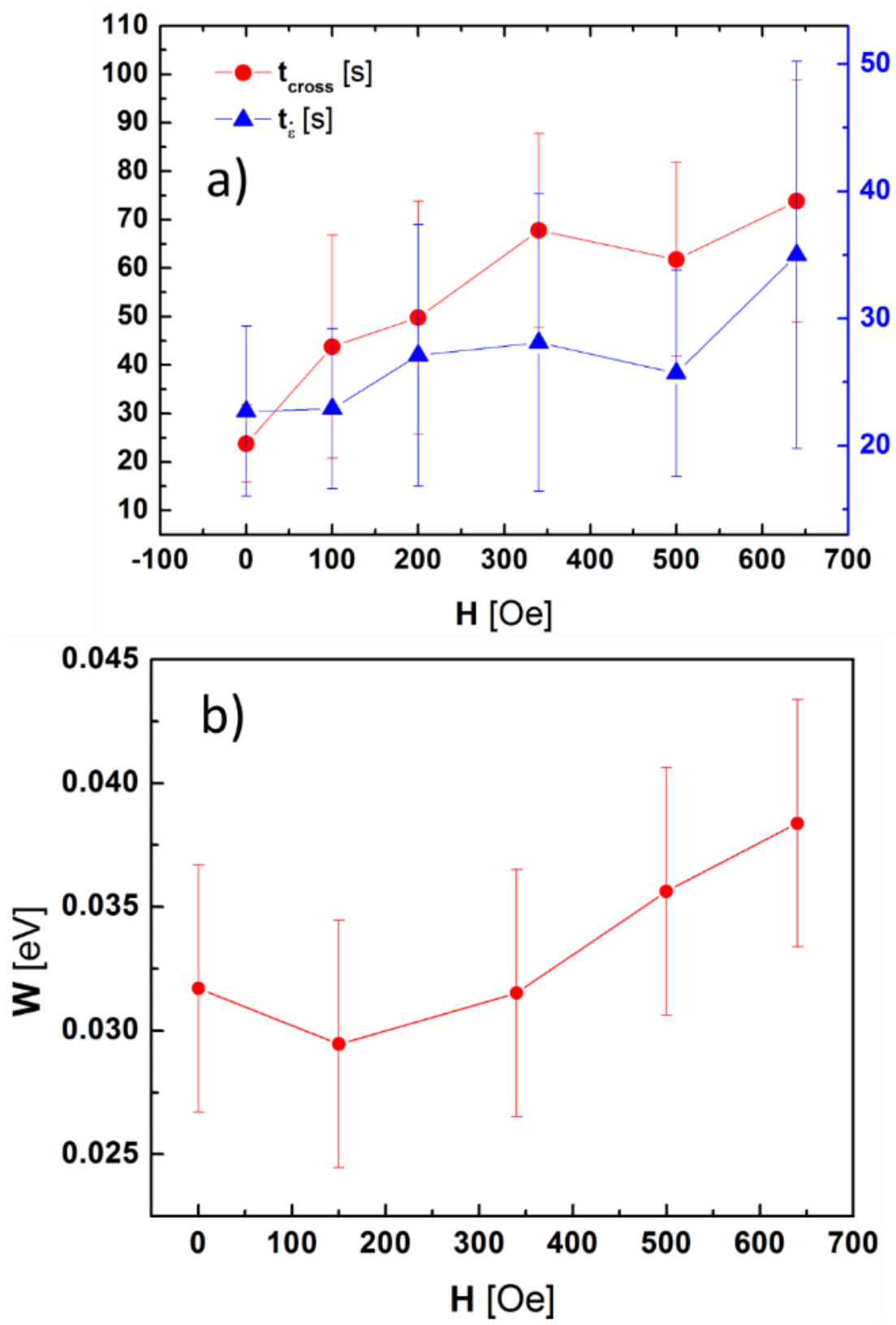

Figure $\mathbf{5 . 2 6}$ (a) Dependence of $t_{\text {cross }}$ and $t_{\dot{\varepsilon}}$ with the magnetic field intensity applied along the width direction $(\theta=0, \phi=0)$ calculated from creep measurements on 2605SA1 for a fixed temperature $\frac{T}{T_{g}}=0.8$ and stress $\sigma=25$ MPa. (b) $W$ as a function of magnetic field intensity applied along the width direction $(\theta=0, \phi=0)$ calculated from creep measurements on 2605SA1 for a fixed temperature $\frac{T}{T_{g}}=0.8$ and stress $\sigma=25 \mathrm{MPa}$. 


\subsubsection{The influence of magnetic field orientation on creep deformation: azimuthal angle $\theta$}

In this subsection the influence of the magnetic field orientation within a plane perpendicular to the stress field is analyzed. Such perpendicular plane to the direction of the mechanical stress is characterized by the constrain $\phi=0$, as defined in Chapter 3. The orientation within that plane can be described by the angle $\theta$, which ranges from $\theta=0$ in the case that the magnetic field is applied along the width of the sample, to $\theta=90$ which represents a magnetic field applied along the thickness of the ribbon. Figure 5.27 depicts schematically the magnetic field orientation during the experiments described in this subsection.

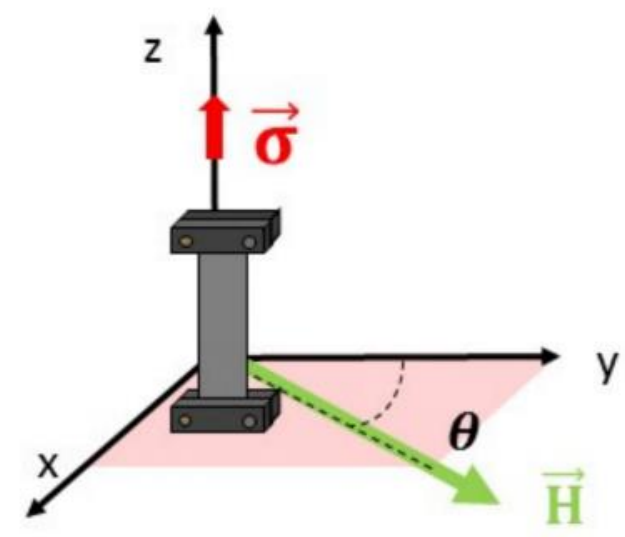

Figure 5.27 Schematic view of the magnetic field orientation with respect to the mechanical stress and ribbon axes used in the experiments described in the current subsection

In order to explore the influence of the angle $\theta$, a set of creep measurements were performed keeping constant the stress, temperature, and intensity of magnetic field $\left(\frac{\mathrm{T}}{\mathrm{T}_{\mathrm{g}}}=0.8, \sigma=\right.$ $15 \mathrm{MPa},|\vec{H}|=500 \mathrm{Oe}$ ) and varying such angle.

Figure 5.28 (a) displays the creep curves for this set of experiments and Figure 5.28(b) present the waiting time distributions of the creep curves shown in Figure 5.28(a). A power law regime can be appreciated for each of the experiments, as well as a higher slope for short waiting times. 

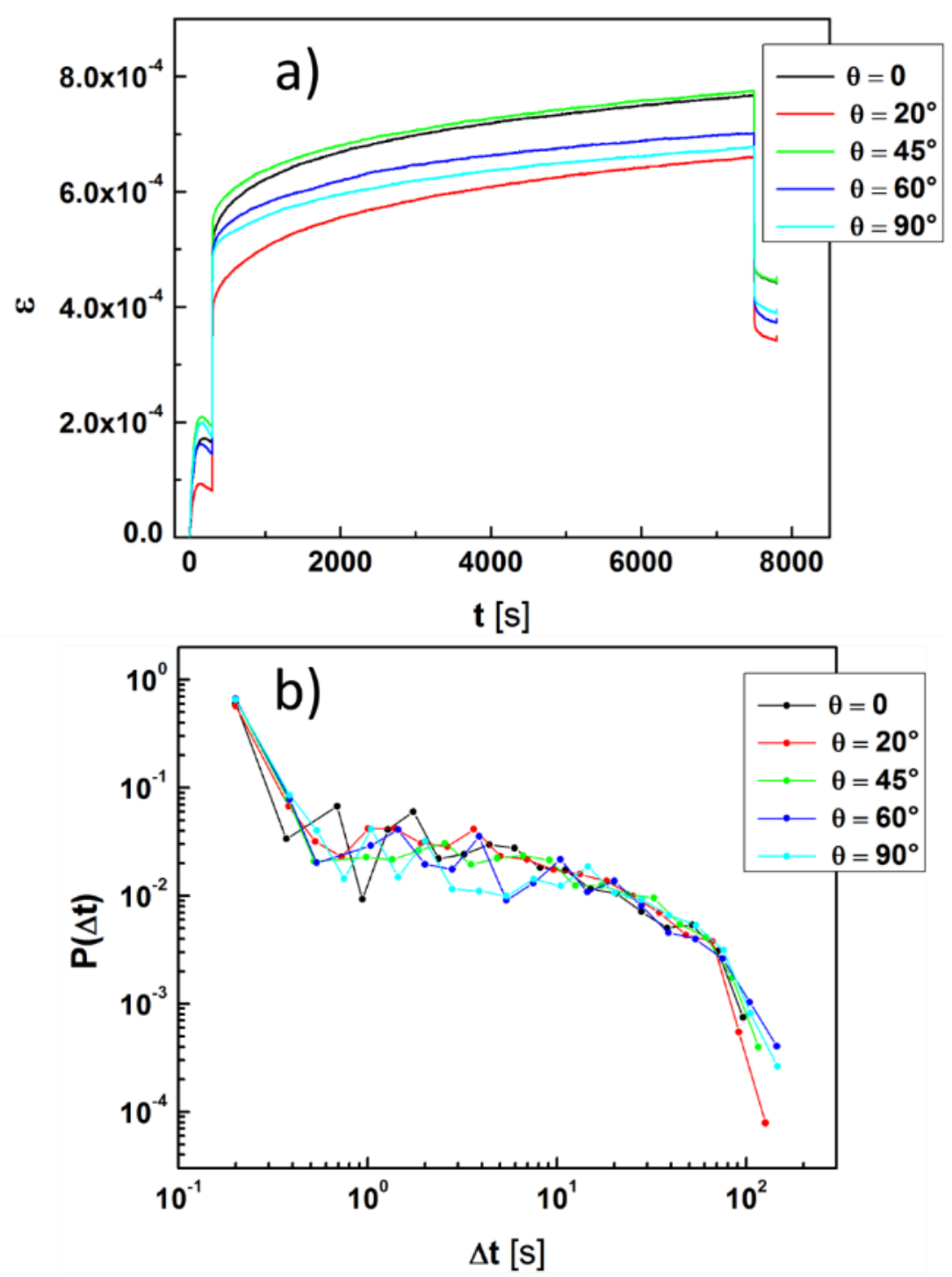

Figure 5.28 (a) Creep measurements of 2605SA1 performed at $\frac{\mathrm{T}}{\mathrm{T}_{\mathrm{g}}}=0.8, \sigma=$ $15 \mathrm{MPa},|\vec{H}|=5000 e, \phi=0$ ) for different angles $\theta$. (b) Double logarithmic representation of the waiting time distribution calculated from the creep measurements shown in (a)

Figure 5.29 shows the waiting time distribution splitting the data between before and after the crossover time $t_{\text {cross }}$. It can be seen in Figure 5.29 (a)-(e) that there is a significant change of slope at $t_{\text {cross }}$, but the power law shape is well preserved in each of the cases. Figure 5.29(f) shows the fit of the experimental power laws. The experimental exponents before $\left(\tau_{1}\right)$ and after $\left(\tau_{2}\right)$ the crossover oscillate around the values $\tau_{1}=-1.5 \pm 0.2$ and $\tau_{2}=-0.8 \pm 0.2$ respectively. 

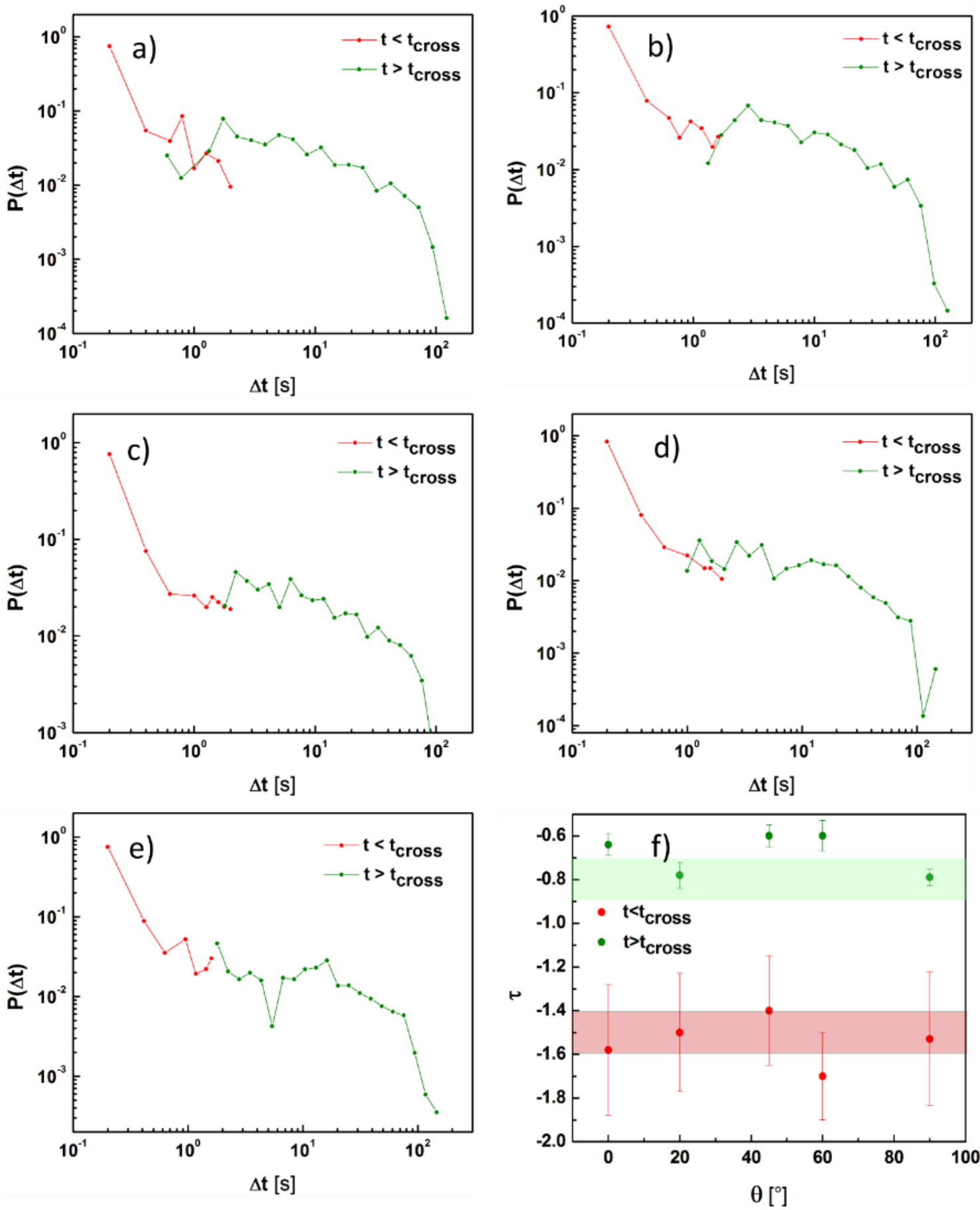

Figure 5.29 Waiting time distributions before and after the crossover time, for the creep measurements of 2605SA1 performed at $\frac{T}{T_{g}}=0.8, \sigma=15 \mathrm{MPa},|\vec{H}|=$ $5000 e, \phi=0$ ) for different angles $\theta$. (a) $\theta=0$. (b) $\theta=20^{\circ}$. (c) $\theta=45^{\circ}$. (d) $\theta=$ $60^{\circ}$. (e) $\theta=90^{\circ}$. (f) Best fit of the power law exponents before and after the crossover calculated from the curves (a)-(e), the coloured areas represent the intervals $\tau_{1}=-1.5 \pm 0.1$ and $\tau_{2}=-0.8 \pm 0.1$.

The three figures of merit, $t_{\text {cross }}, t_{\dot{\varepsilon}}$ and $W$ are shown in Figure 5.30. It can be observed how all of them decrease their magnitude as the angle shifts from $\theta=0$ towards $\theta=90^{\circ}$. That change in the angle $\theta$ corresponds to a rotation from the width direction towards the thickness direction of the 
sample. Since the anisotropy along the out-of-plane direction is much higher than along the sample width, a decrease in the magnetic field effect is expected as the angle approaches $\theta=90^{\circ}$. These results are discussed further in the chapter 6.
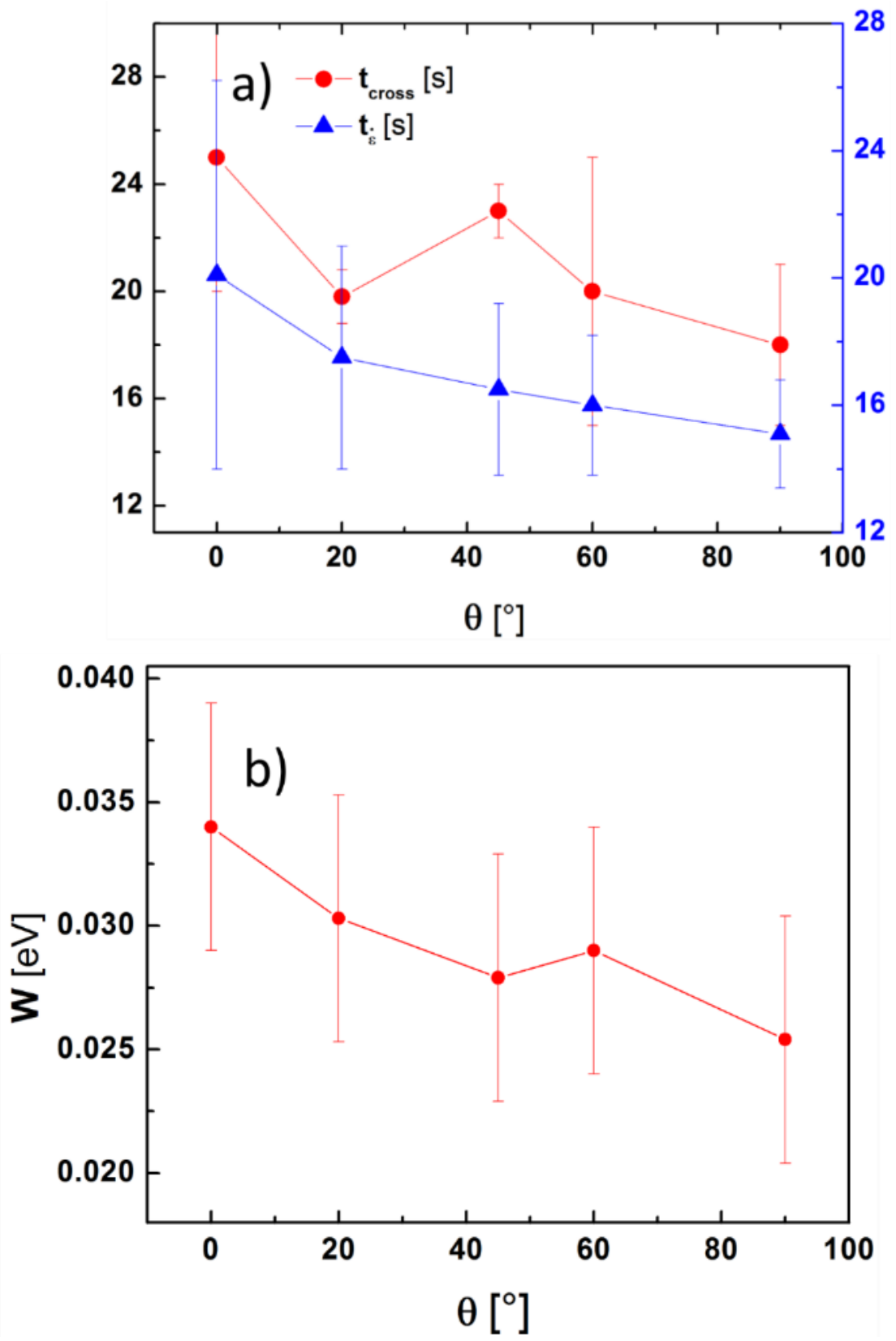

Figure 5.30 (a) Dependence of $t_{\text {cross }}$ and $t_{\dot{\varepsilon}}$ with the angle $\theta$ for creep experiments performed on 2605SA1 at, $\left.\frac{T}{T_{g}}=0.8, \sigma=15 \mathrm{MPa},|\vec{H}|=500 \mathrm{0e}, \phi=0\right)$.(b) $W$ as a function of the angle $\theta$ for creep experiments performed on 2605SA1 at , $\frac{T}{T_{g}}=$ $0.8, \sigma=15 \mathrm{MPa},|\vec{H}|=500$ Oe, $\phi=0$ 


\subsubsection{The influence of magnetic field orientation on creep deformation: polar angle $\phi$}

The influence of the angle between the magnetic and stress field is analyzed in this subsection. In order to do that, the angle $\theta$ is fixed to $\theta=0$, and the angle $\phi$ between the stress and magnetic field is swept from experiment to experiment making use of a magnet holder feed with a couple of AINiCo cylindrical magnets. Thus, the set of creep measurements shown below were performed keeping constant the stress, temperature, and intensity of magnetic field $\left(\frac{\mathrm{T}}{\mathrm{T}_{\mathrm{g}}}=0.8, \sigma=\right.$ $15 \mathrm{MPa},|\overrightarrow{\mathrm{H}}|=120 \mathrm{Oe}$ ) and varying the angle $\phi$. Figure 5.31 illustrates schematically the orientation of the magnetic field used during the experiments shown in this subsection.

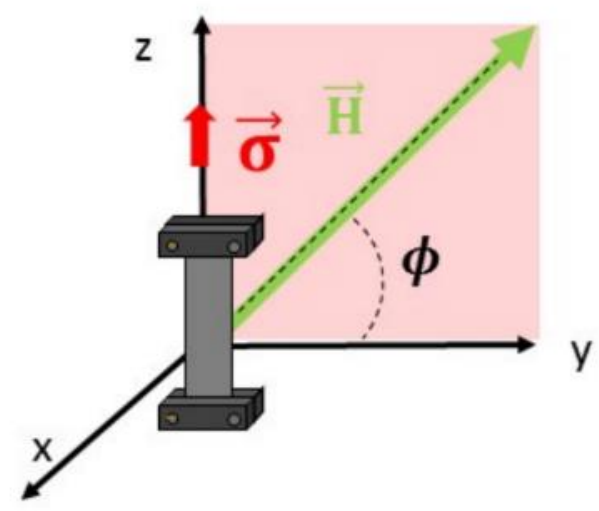

Figure 5.31 Schematic view of the magnetic field orientation with respect to the mechanical stress and ribbon axes used in the experiments shown in the current subsection

Figure 5.32 (a) displays the creep curves for this set of experiments. Although the elastic deformation varies between each measurement, no clear change in the slope during the anelastic deformation can be appreciated from a first inspection of that figure. Figure 5.32(b) presents the waiting time distributions of the creep curves shown in Figure 5.32(a).The waiting time distribution decay in every case with a power law fashion, and a crossover can be appreciated for short waiting times. 

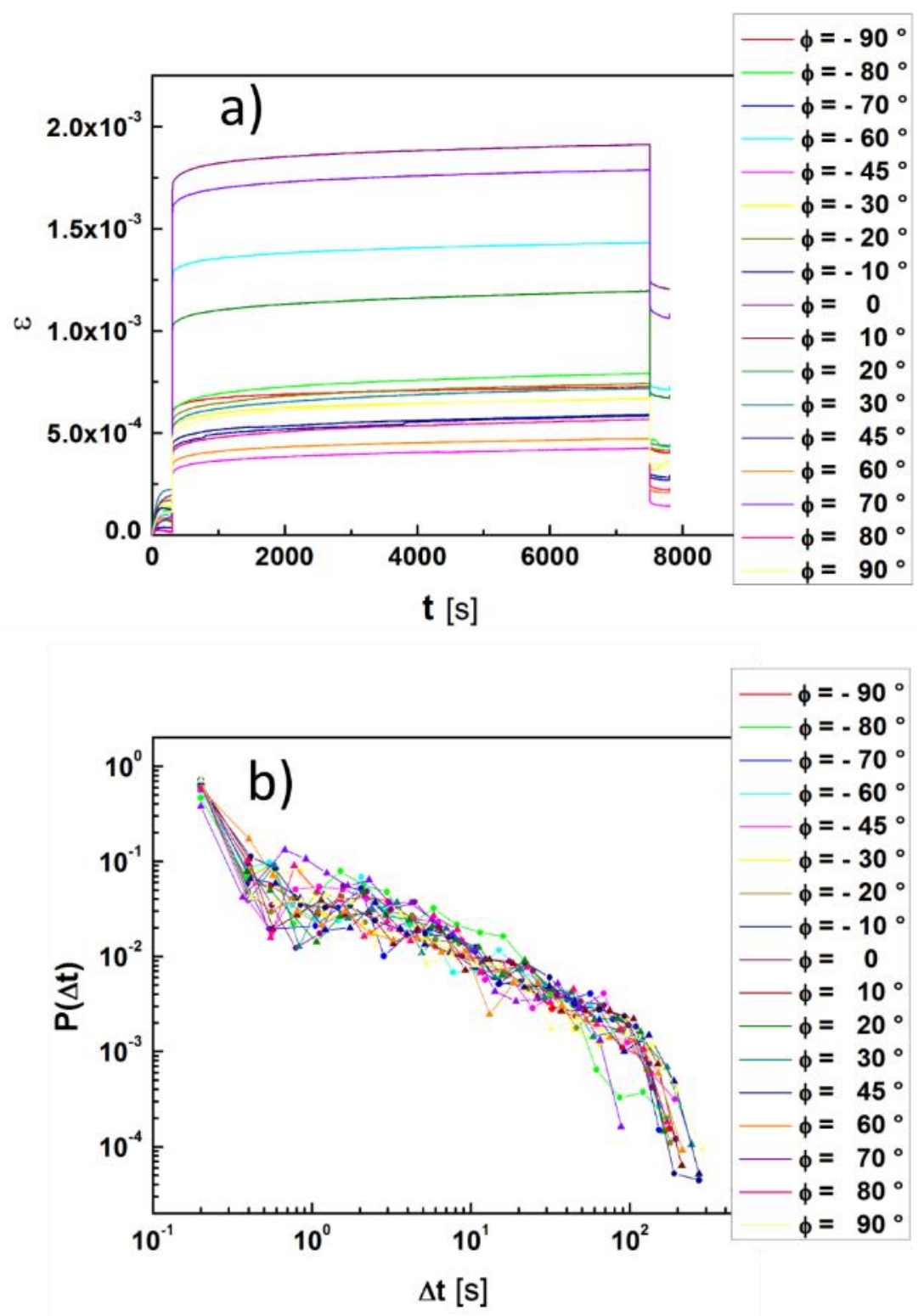

Figure 5.32 (a) Creep measurements of 2605SA1 performed at $\frac{T}{T_{g}}=0.8, \sigma=$ $15 \mathrm{MPa},|\vec{H}|=120$ Oe, $\theta=0$ ) for different angles $\phi$. (b) Double logarithmic representation of the waiting time distribution calculated from the creep measurements shown in (a)

Figure 5.33 shows the waiting time distribution splitting the data between before and after the crossover time $t_{\text {cross }}$, for some of the creep measurements presented in Figure 5.32. Figure 5.33(a)(e) show a significant change of slope at $t_{\text {cross }}$, and a power law shape which is well preserved in each of the cases. Figure 5.33.(f) shows the fit of the experimental power laws. The exponents of the waiting time distributions after the crossover $\left(\tau_{2}\right)$ have all values in the range $\tau_{2}=-0.8 \pm 0.1$. In the case of the first regime, the experiments performed at angles between $\phi=-45,45$ show exponents in the range $\tau_{1}=-1.5 \pm 0.1$. However, as the magnetic field approaches a parallel orientation with respect to the stress $(\phi= \pm 90-80)$, there is a significant shift of the exponents $\tau_{1}$, which approach values from $\tau_{1}=-1.8$ up to -2 . 

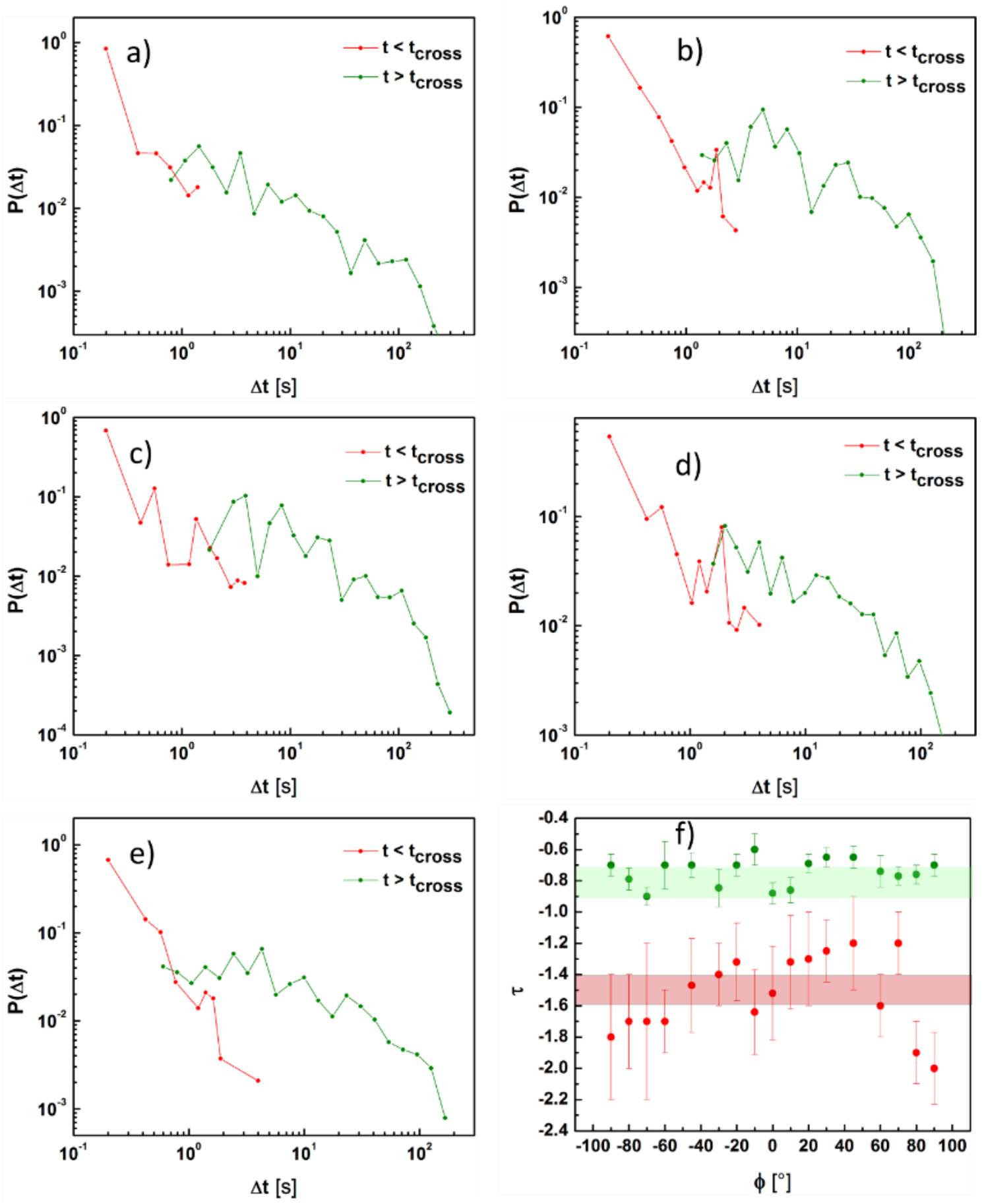

Figure 5.33 Waiting time distributions before and after the crossover time calculated from creep measurements of 2605SA1 performed at $\frac{T}{T_{g}}=0.8, \sigma=$ $15 \mathrm{MPa},|\vec{H}|=120 O e, \theta=0$ ) for different angles $\phi$. (a) $\phi=-90$ Oe. (b) $\phi=$ $-60^{\circ}$. (c) $\phi=-30^{\circ}$. (d) $\phi=30^{\circ}$. (e) $\phi=90^{\circ}$. (f) Power law exponent fit before and after the crossover calculated from the curves (a)-(e). Coloured areas represent the intervals $\tau_{1}=-1.5 \pm 0.1$ and $\tau_{2}=-0.8 \pm 0.1$.

The evolution of the three figures of merit, $t_{\text {cross }}, t_{\dot{\varepsilon}}$ and $W$ with the angle $\phi$ is shown in Figure 5.34. Despite the fluctuation of the data, it can be observed in Figure 5.34.(a) that the crossover times approach minimum values when the stress and magnetic field are parallel aligned 
$\left(|\phi| \rightarrow 90^{\circ}\right)$. The rest of the values of $t_{\text {cross }}$ show a small $\phi$-dependence, and fluctuate around an average value. That average value increases for the values $\phi=30,-60$ in which the crossover time is increased. The values of $t_{\dot{\varepsilon}}$ follow a similar tendency, although in this case the dispersion in the data is bigger.

The evolution of $W$ shown in Figure 5.34(b) share some features with the evolution of $t_{\text {cross }}(\phi)$ shown in Figure 5.34(a). $W$ oscillates around a mean value for all the experimental conditions except for $\phi=-45,60$ in which two sharp peaks can be distinguished. Such behavior is discussed in terms of the magnetoelastic coupling and the orientation of domain walls with respect to the Shear Transformation Zones in Chapter 6.
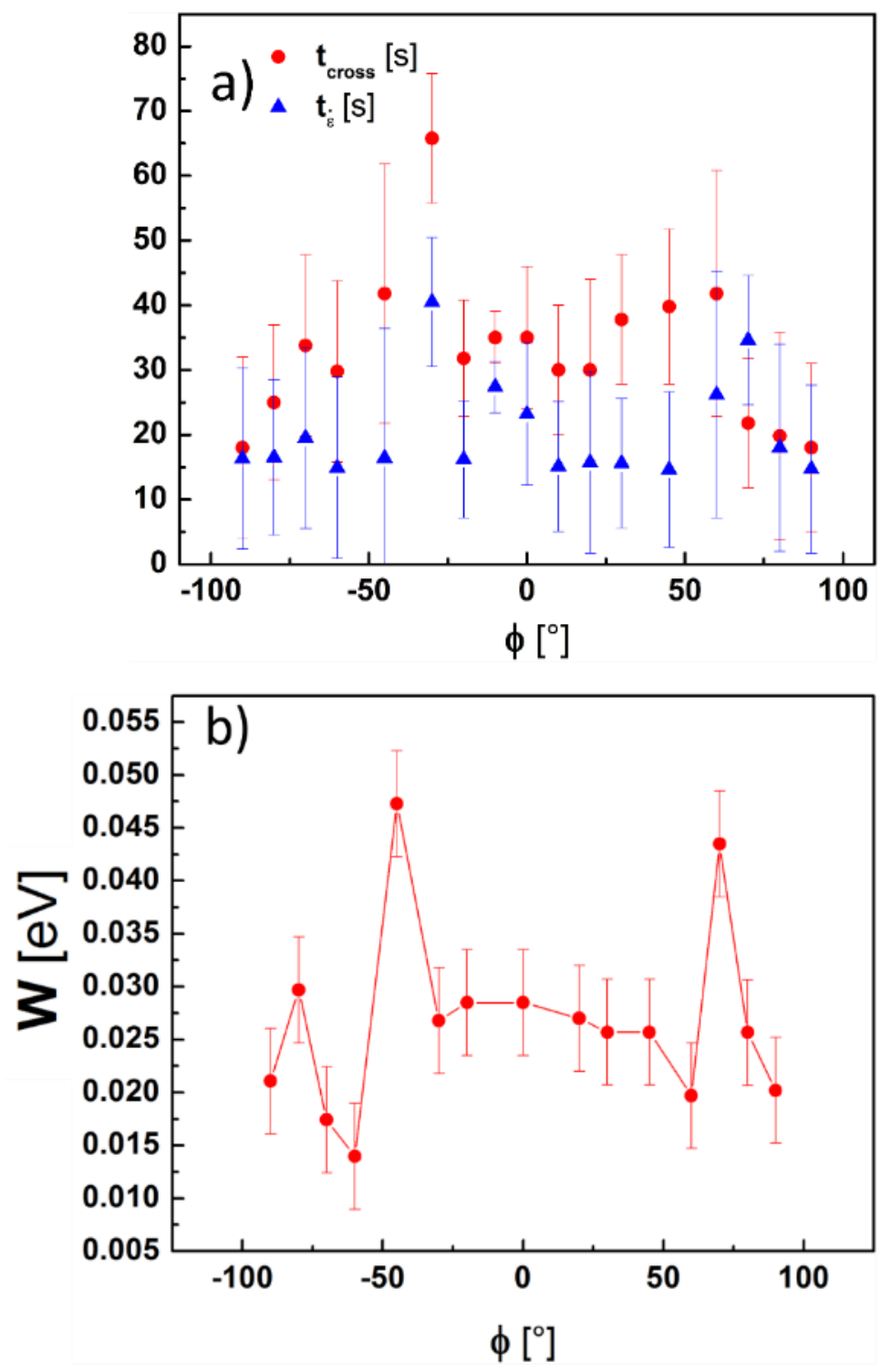

Figure $\mathbf{5 . 3 4}$ (a) Dependence of $t_{\text {cross }}$ and $t_{\dot{\varepsilon}}$ with the angle $\phi$ for creep experiments performed on 2605SA1 at, $\frac{T}{T_{g}}=0.8, \sigma=15 \mathrm{MPa},|\vec{H}|=1200 e, \theta=0$ ). (b) $W$ as a function of the angle $\theta$ for creep experiments performed on 2605SA1 at , $\frac{T}{T_{g}}=$ $0.8, \sigma=15 \mathrm{MPa},|\vec{H}|=120 \mathrm{Oe}, \theta=0$ ) 


\subsection{Stress-strain measurements of magnetic glasses under magnetic driving}

The current section focuses on the analysis of stress-strain curves under both magnetic and mechanical driving. The motivation to perform these experiments was that they could be done at room temperature, which is not the case for creep experiments since a minimum temperature of $\mathrm{T}=540 \mathrm{~K}$ is required in order to measure appreciable deformation flow in the magnetic compositions. This fact, makes it possible to use $N d$ magnets and therefore increase the available field intensity range until 1000 Oe. Additionally, since the magnetization decreases with temperature, the coupled magneto-mechanical effects are expected to be maximized at low temperatures.

All the experiments in this section were performed using two different alloys: a magnetostrictive 2605SA1, with magnetostriction constant $\lambda_{s}=27 \times 10^{-6}$, and a $\mathrm{Fe}_{2} \mathrm{Co}_{73} \mathrm{Si}_{10} \mathrm{~B}_{15}$ metallic glass with vanishing magnetostriction. The purpose of choosing these two alloys is to analyze the influence of the magnetostriction on the magneto elastic coupling effects.

Figure 5.35 displays the EDX spectra of the Co-rich alloy. It presents emission lines corresponding to $\mathrm{Co}, \mathrm{Fe}$ and $\mathrm{Si}$. Table 5.3 shows the element atomic percentage of such alloy calculated from the quantitative analysis of such spectra. It must be noted that in this technique, the emission lines of the light elements like B cannot be resolved. EDX analysis reveals that $\mathrm{Co}$ is the major magnetic component in this alloy.

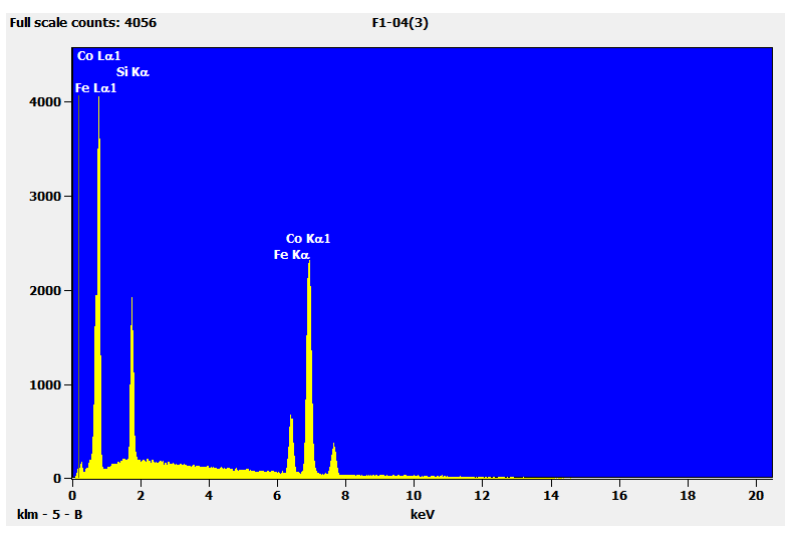

Figure 5.35 EDX spectra of $\mathrm{Fe}_{2} \mathrm{Co}_{73} \mathrm{Si}_{10} \mathrm{~B}_{15}$

Table 5.3 Quantitative analysis of the $\mathrm{Fe}_{2} \mathrm{Co}_{73} \mathrm{Si}_{10} \mathrm{~B}_{15}$ spectra.

\begin{tabular}{|c|c|}
\hline Element & Atomic percentage [\%] \\
\hline $\mathrm{Co}$ & $71.8 \pm 0.7$ \\
\hline $\mathrm{Fe}$ & $14.6 \pm 0.4$ \\
\hline $\mathrm{Si}$ & $13.6 \pm 0.2$ \\
\hline
\end{tabular}


The magnetic properties of the Co-rich glass were characterized measuring the hysteresis loops by means of Vibrating Sample Magnetometer (VSM) at room temperature, as described in section 3. Figure 5.36(a) shows the hysteresis loops measured along the main axes of symmetry of a Co-rich ribbon. The coercive field measured along each orientation is very low with values in the order $\mathrm{H}_{c}=$ $10 \pm 5$ Oe. Anisotropy fields along width and length orientations can be estimated from the hysteresis loops to be $\mathrm{H}_{\mathrm{A}}^{\mathrm{L}}=105 \pm 10$ and $\mathrm{H}_{\mathrm{A}}^{\mathrm{W}}=360 \pm 20$ Oe. Figure 5.36(b) shows a DSC scan of the Co-rich alloy measured with a heat rate $\dot{T}=20 \mathrm{~K} / \mathrm{min}$. Crystallization takes place at the temperature $\mathrm{T}_{\mathrm{x}}=822 \pm 5 \mathrm{~K}$. On the other hand no clear signature of either the glass or the ferromagnetic-paramaganetic transitions can be observed in the DSC scan.
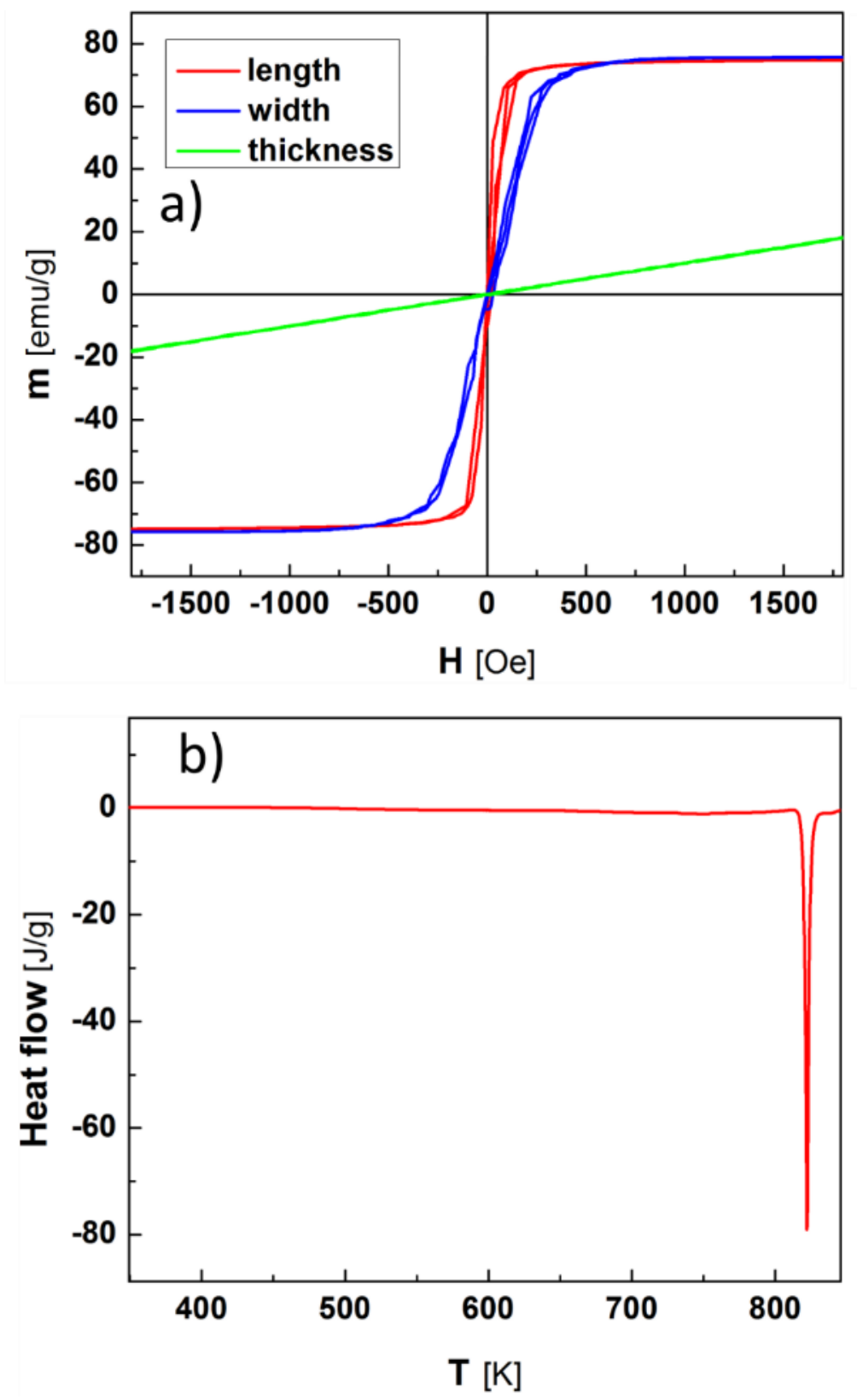

Figure $\mathbf{5 . 3 6}$ (a) Hysteresis loops measured along the three main axes of symmetry of the Co-rich alloy by means of Vibrating Sample Magnetometer. (b) Differential Scanning Calorimeter measurement of the Co-rich alloy. 
After each of the measurements, the structure of the samples was investigated through XRD experiments to make sure that the structure remained amorphous. Figure 5.37 shows a sample of XRD spectra for both compositions. It can be seen that none of them crystallized during the experiments, which would have been unlikely given that they were conducted at room temperature.
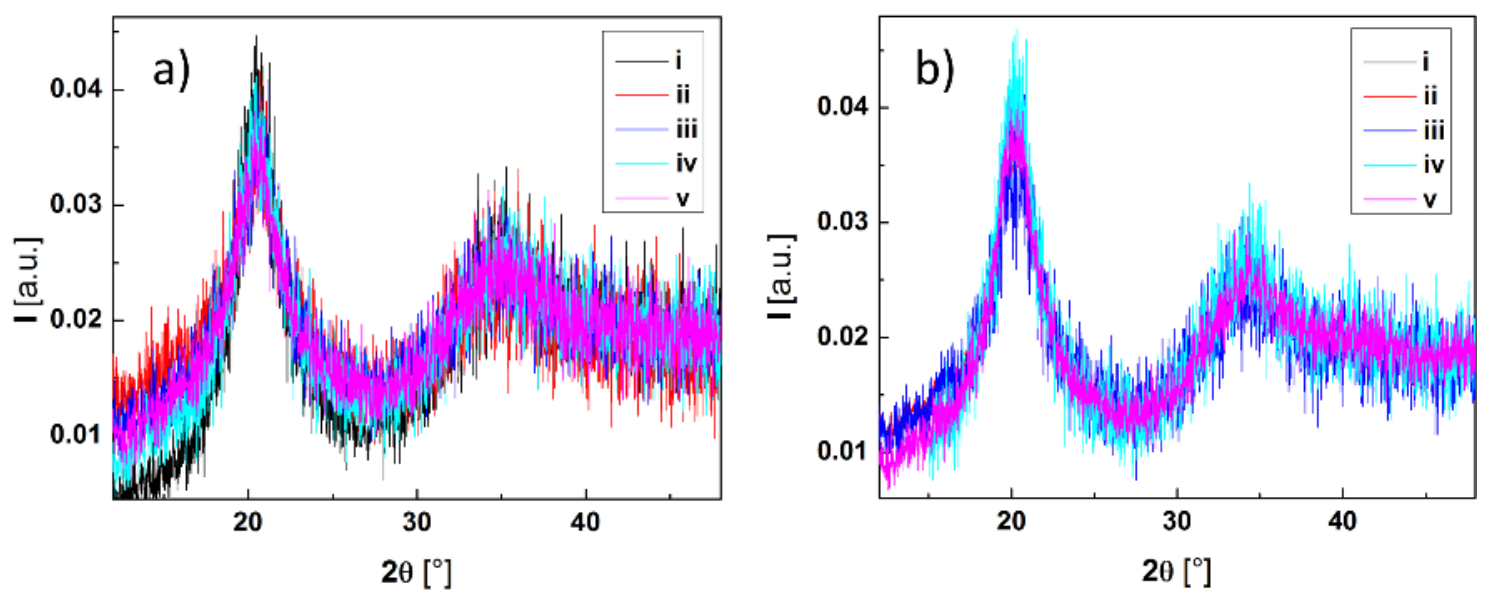

Figure 5.37 (a) XRD spectra of the Co-rich alloy. i) As cast. ii) Stressed under field $|\vec{H}|=340$ Oe. iii) Stressed under field $|\vec{H}|=500$ Oe. iv) Stressed under field $|\vec{H}|=$ 700 Oe. v) Stressed under field $|\vec{H}|=950$ Oe. (b) XRD spectra of the Fe-rich alloy. i) As cast. ii) Stressed under field $|\vec{H}|=340$ Oe. iii) Stressed under field $|\vec{H}|=500$ Oe. iv) Stressed under field $|\vec{H}|=700$ Oe. v) Stressed under field $|\vec{H}|=950$ Oe

With the aim to study the effect of a magnetic field in the local mechanical response of magnetic metallic glasses, tensile stress-strain tests were performed on both alloys under several magnetic field intensities ranging from $\mathrm{H}=0-1000$ Oe and in two different orientations $\left(\theta=0\right.$ and $\left.\theta=90^{\circ}\right)$ at a controlled temperature $\mathrm{T}=300 \mathrm{~K}$.

The strain produced by a freshly prepared amorphous sample that was subjected to a stress ramp protocol ranging from $\sigma=10$ to $30 \mathrm{MPa}$ at a Force rate of $30 \mathrm{mN} / \mathrm{min}$ is measured for each of the experimental conditions (fixed $|\vec{H}|, \theta$ and composition). The ramp is run from $10 \mathrm{MPa}$ in order to ensure that the sample stays straight during the whole run. Each protocol was repeated four times to collect more data and improve the statistics of further analysis. Figure 5.38 show the stress-strain curves for all the different experimental conditions. Figure 5.38(a)-(b) show the result of the stressstrain test performed with the magnetic field applied along the out of plane direction $\left(\theta=90^{\circ}\right)$ for the Co-rich and Fe-rich alloys respectively. With the magnetic field applied in such geometry, no clear change of slope can be appreciated in the stress-strain curves. Figure 5.38(c) shows the experimental stress-strain curves corresponding to a magnetic field applied along the width direction $(\theta=0)$ of the non magnetostrictive Co-rich alloy. It can be seen that in this case, the slope of the stress-strain curves increases slightly as the magnetic field intensity increases. A more dramatic effect can be appreciated in the case of the magnetostrictive Fe-rich alloy exposed to a magnetic field along the width direction $(\theta=0)$. Figure 5.38(d) shows that in that case, for magnetic field intensities between $|\vec{H}|=340-700$ Oe, the stress-strain curves are separated in two regions by a kink located at a certain stress $\sigma_{k}$. The first region $\left(\sigma<\sigma_{k}\right)$ is characterized by a lower slope, which corresponds to a lower Young modulus. The slope of the second region 
$\left(\sigma>\sigma_{k}\right.$ ) is much higher, which corresponds to Young modulus value closer to the corresponding to the non-magnetized sample. The stress at which this kink takes place $\left(\sigma_{k}\right)$, shifts to higher values as the field intensity is increased, until the whole effect vanishes when a field of $|\vec{H}|=950$ Oe is applied.
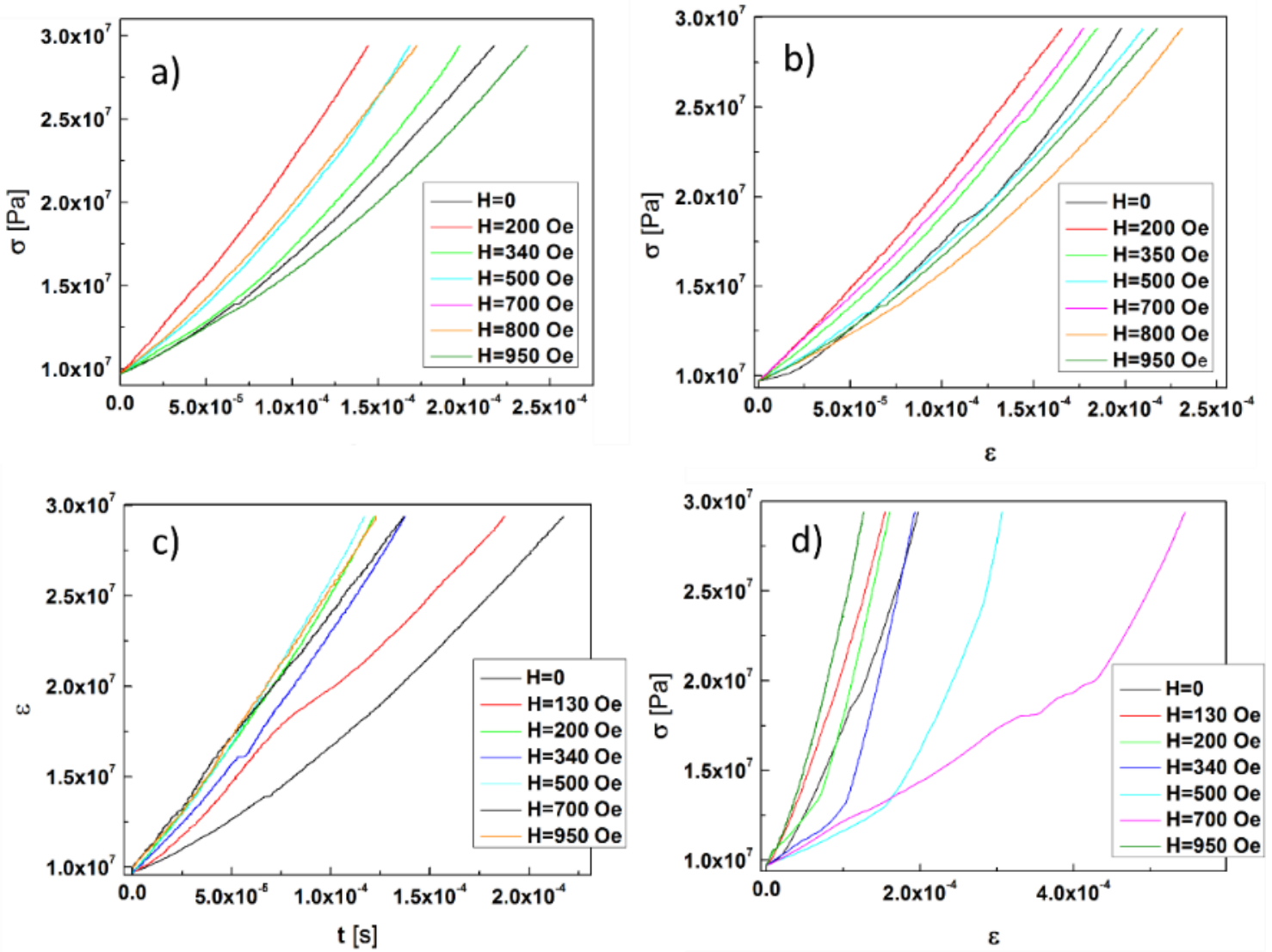

Figure 5.38 (a) Stress-strain curves of $\mathrm{Fe}_{2} \mathrm{Co}_{73} \mathrm{Si}_{10} \mathrm{~B}_{15}$ under different intensities of magnetic field applied along the out-of-plane direction $\left(\theta=90^{\circ}\right)$. (b) Stress strain curves of 2605SA1 under different intensities of magnetic field applied along the out-of-plane direction $\left(\theta=90^{\circ}\right)$. (c) Stress-strain curves of $\mathrm{Fe}_{2} \mathrm{Co}_{73} \mathrm{Si}_{10} \mathrm{~B}_{15}$ under different intensities of magnetic field applied along the width direction $(\theta=0)$. (d) Stress-strain curves of 2605SA1 under different intensities of magnetic field applied along the width direction $(\theta=0)$. All test are perfomed with a force rate of 30 $\mathrm{mN} / \mathrm{min}$ and at a constant temperature $\mathrm{T}=300 \mathrm{~K}$.

The influence of the magnetic field in the macroscopic Young modulus is summarized in Figure 5.39. Figure 5.39(a) displays the evolution of the macroscopic Young modulus as a function of the applied field applied along the directions $\theta=0$ and $\theta=90^{\circ}$ for the Co-rich alloy and Figure 5.39 (b) shows the analogous information in the case of the magnetostrictive Fe-rich alloy. In both cases the macroscopic Young modulus is calculated by the linear fit of the stress-strain curves shown in Figure 5.38 .

A first inspection of the curves shown in Figure 5.39 reveal that the Young modulus do not seem to be affected by the magnetic field when it is applied along the out-of-plane direction $\left(\theta=90^{\circ}\right)$. As it will be discussed in Chapter 6 , that is an expected result since the susceptibility of ribbon samples 
along the out-of-plane direction is almost negligible, and therefore the sample almost does not change it magnetic state when subjected to a magnetic field along that direction. However, the data corresponding to the magnetic field applied along the $\theta=0$ direction reveal important changes in the Young modulus. The Co-rich alloy shows an increase of the Young modulus for magnetic fields $|\vec{H}|>200$ Oe, the change of the Young modulus takes place in the order of $50 \%$ and it remains roughly constant for all the range of higher magnetic fields. On the other hand the magnetostrictive Fe-rich alloy show a dramatic decrease of the Young modulus for magnetic fields in the interval $|\vec{H}|=340-700$ Oe. This is the so called $\Delta E$-effect ${ }^{14,110}$, and in this case the decrease of the Young modulus reaches values higher than the $50 \%$.
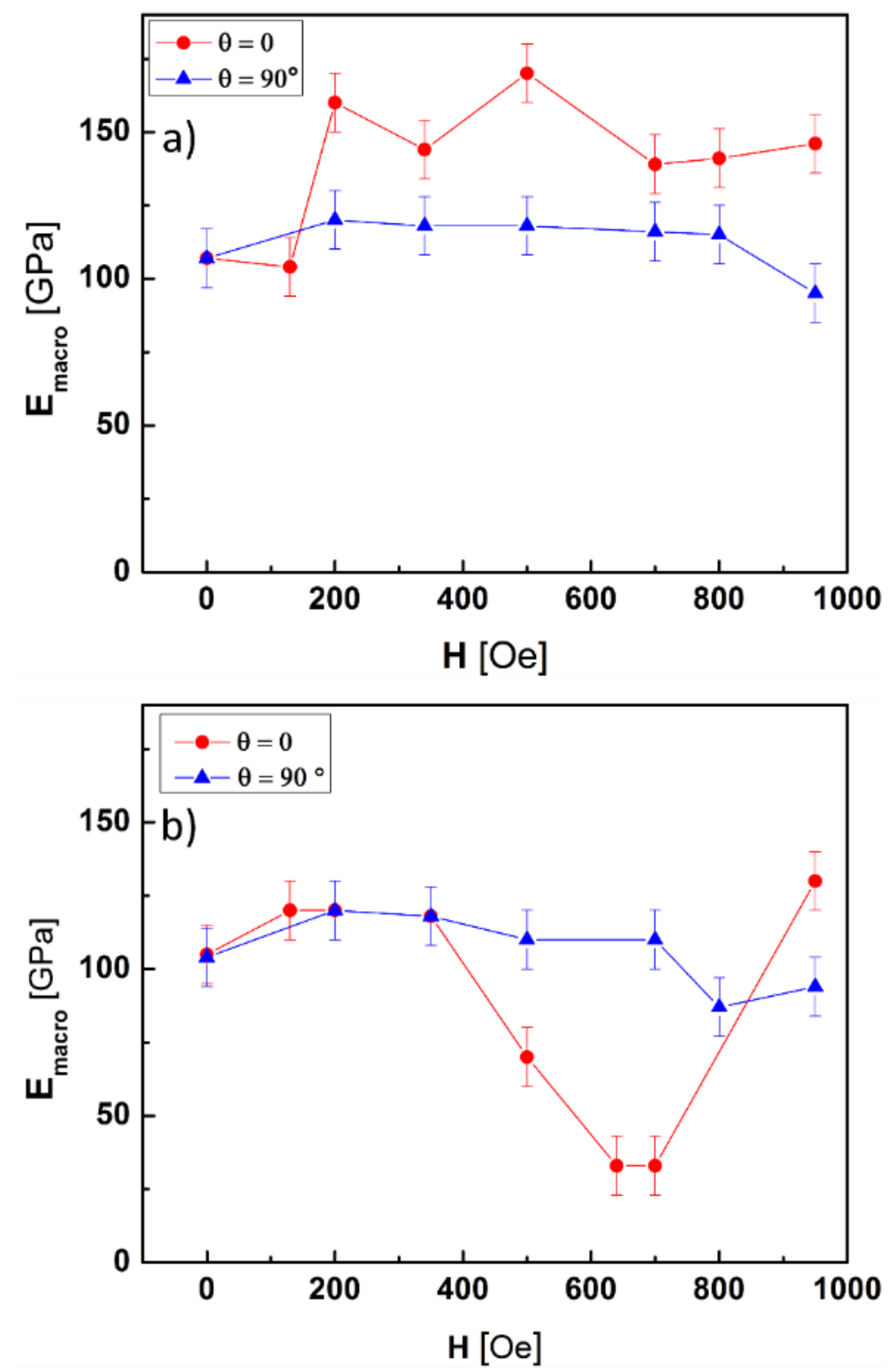

Figure 5.39 (a) Macroscopic Young modulus $E_{\text {macro }}$ as a function of the intensity of magnetic field for the CoFeSiB metallic glass. (b) Macroscopic Young modulus $E_{\text {macro }}$ as a function of the intensity of magnetic field for the2605SA1 metallic glass.

Figure 5.40 shows the statistical analysis of the intermittency on the stress-strain curves. As described in Chapter 4, the fine structure of the stress-strain curves can analyzed as an array of 
effective modulus $E_{\text {eff }}(\sigma)$. Then, a normalized modulus can be defined as $\hat{E}=\frac{E_{\text {macro }}}{E_{\text {eff }}}$. In a stress controlled experiment, mechanical avalanches would show up as strain bursts, which would induce an instantaneous reduction of the effective modulus $E_{\text {eff }}$. Therefore, the definition of $\hat{E}$ allows to identify the avalanche behavior with the condition $\hat{E}>1$.
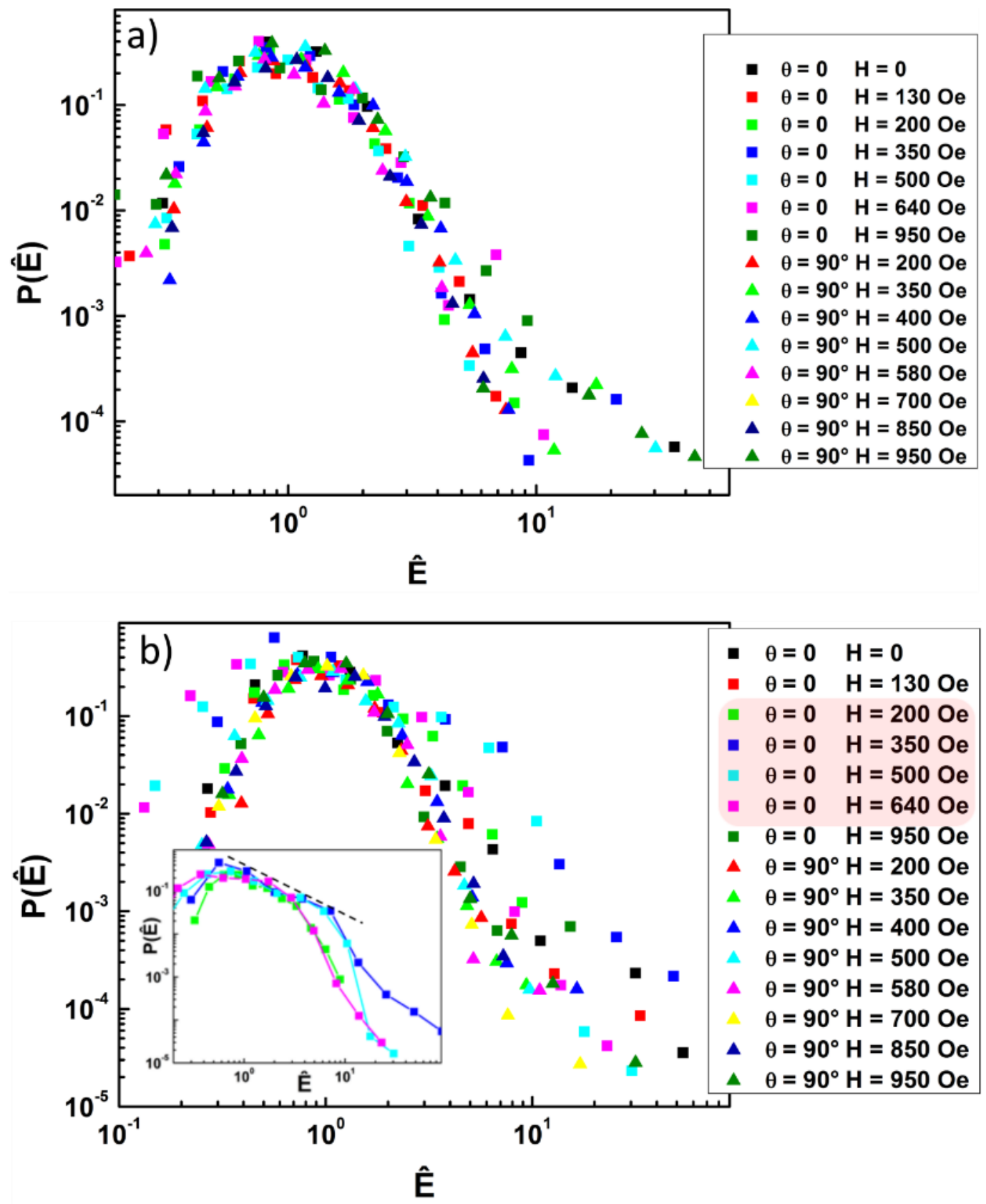

Figure 5.40 (a) Distribution of normalized modulus $P(\hat{E})$ of $\mathrm{Fe}_{2} \mathrm{Co}_{73} \mathrm{Si}_{10} \mathrm{~B}_{15}$ under magnetic driving (b) Distribution of normalized modulus $P(\hat{E})$ of $2605 \mathrm{SA} 1$ under magnetic driving. Figure $\mathbf{5 . 4 0}$ (b) Inset. $P(\widehat{E})$ for $\theta=0,|\vec{H}|=200,340,500$ and 640 Oe.

Squares represent experiments measured under magnetic field applied along $\theta=0$ and triangles along $=90^{\circ} 168$.

Figure 5.40(a) shows the probability distribution of the normalized modulus for the Co-rich alloy exposed to the different magnetic field conditions. It can be seen that all the experiments collapse in a master plot which presents a symmetric peak around $\widehat{E}=1$. Data shown in Figure 5.40 (b) 
corresponds to the magnetostrictive alloy. While most of the measurements on this alloy collapse as well in a master plot, few of them deviate from the collapse and it distribution appear to show a certain regime of power-law scaling, as it is shown in the inset of Figure 5.40(b). The experiments whose distributions do not collapse with the others correspond with the conditions in which the $\Delta E$-effect is maximized. This result is discussed in detail in the next chapter.

Figure 5.41.(a) shows the non-normalized distribution of effective modulus $E_{\text {eff }}$ corresponding to the 2605SA1 alloy under magnetic loading along $\theta=0$. It can be seen that as the magnetic field intensity increases, a left peak emerges which corresponds to a Young modulus $E \sim 25 \mathrm{GPa}$. The height of this peak increases with the magnetic field and reaches a maximum at the condition $|\vec{H}|=$ 700 Oe. This peak disappears when the field intensity reaches the value $|\vec{H}|=950$ Oe. Figure 5.41(b) shows the distribution of effective modulus of 2605SA1 subjected to a magnetic field of $\mathrm{H}=500 \mathrm{Oe}$, separated between the modulus measured before and after the kink located at $\sigma_{k}$ in the stress-strain curve. It shows that the low moduli are located almost uniquely before the kink. After the kink the distribution of effective modulus is centered around $\mathrm{E} \sim 100 \mathrm{GPa}$, which corresponds to the Young modulus of the demagnetized sample. These results are discussed in detail in Chapter 6.
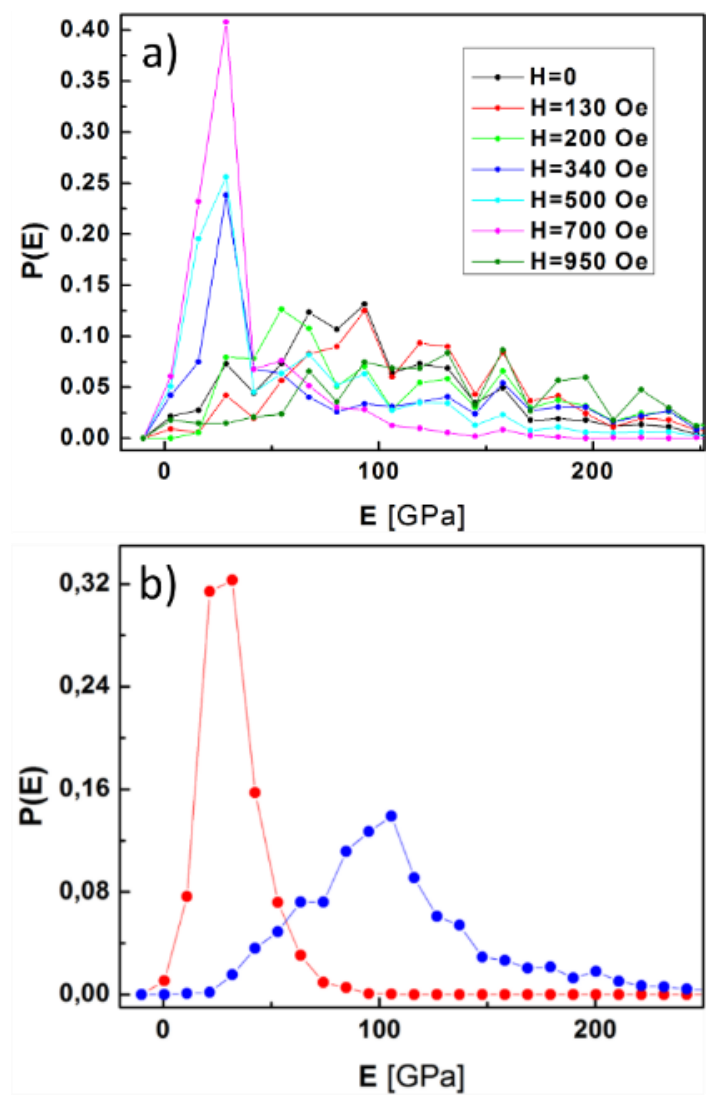

Figure 5.41 (a) Distribution of effective modulus of the 2605SA1 alloy under magnetic loading along $\theta=0$ for different intensities of applied field. (b) Distribution of effective modulus of 2605SA1, under an applied field of $\mathrm{H}=500 \mathrm{Oe}$ separated in two regimes according to the kink that takes places at $\sigma_{k}=12.5 \mathrm{MPa}$ : $P\left(E_{\text {eff }}\right)$ for $\sigma<\sigma_{k}$ (red) and $P\left(E_{\text {eff }}\right)$ for $\sigma>\sigma_{k}$ (blue) 


\section{Discussion}

\subsection{Creep deformation of a non-magnetic sample}

\subsubsection{Characterization of sample properties}

The study of avalanches produced during creep deformation of non-magnetic samples was performed on $\mathrm{Pd}_{77.5} \mathrm{Cu}_{6} \mathrm{Si}_{16.5}$ ribbons prepared by melt spinning. XRD measurements shown in Figure (5.3) confirmed that the as-cast ribbons were structurally amorphous, and that they remained amorphous after the different creep protocols. Additionally, good match between the nominal composition $\mathrm{Pd}_{77.5} \mathrm{Cu}_{6} \mathrm{Si}_{16.5}$ and the actual element distribution was confirmed by means of EDX measurements shown in Figure 5.1 and Table 5.1. Finally, crystallization and glass transition temperatures were estimated from DSC scans and both values $T_{g}=621 \pm 10 \mathrm{~K}, \mathrm{~T}_{\mathrm{x}}=685 \pm 10 \mathrm{~K}$ are in good agreement with literature values of the same composition ${ }^{30,71,169}$.

\subsubsection{Crossover in the waiting time distribution}

J.O. Krisponeit et al., reported a crossover of the waiting time distribution generated by a $\mathrm{Pd}_{77.5} \mathrm{Cu}_{6} \mathrm{Si}_{16.5}$ ribbon subjected to a creep measurement with experimental conditions $\sigma=15$ $\mathrm{MPa}, \frac{\mathrm{T}}{\mathrm{T}_{\mathrm{g}}}=0.95^{30}$. The exponent of the power law distribution was shown to change from $\tau_{1}=$ $-1.5 \pm 0.2$ for waiting times measured at experimental times $t<100 \min$ to $\tau_{2}=-0.8 \pm 0.2$ in the case of waiting times measured at $t>100 \mathrm{~min}$. Such crossover was associated to a transition in the underlying deformation mechanism from a deformation regime governed by uncorrelated 3dimensional stress-driven plastic events to a regime in which the deformation is mainly carried out by the thermal assisted collective activation of nano-shear bands in 2 dimensions.

The stress-temperature map shown in Figure 5.5 shows the existence of the crossover for a wide range of temperatures and stresses. Moreover, it can be seen that the time $t_{\text {cross }}$ at which such crossover takes place increases as both applied stress and temperature increase. The functional dependence of $t_{\text {cross }}$ with stress and temperature was separately analyzed, and the results were shown in figure 5.8 . 
Additionally, following the method described in Section 4.1.2, the creep measurements were also analyzed from the point of view of the macroscopic strain rate. In that context, the creep curve can be divided into an initial transient characterized by high values of the strain rate $\dot{\varepsilon}$ and its time derivative $\ddot{\varepsilon}$, and a subsequent pseudo-steady state, $t_{\dot{\varepsilon}}$ being the time at which such transition takes place. The clear correlation of $t_{\text {cross }}$ and $t_{\dot{\varepsilon}}$ shown in Figure 5.8. indicates a dependence of the avalanche regime on the overall strain rate ${ }^{35}$.

The dependence of the avalanche regime on the driving rate has been analyzed in detail by $\mathrm{K}$. Martens et al., in the framework of an elasto-plastic model for amorphous systems ${ }^{154}$. By means of sweeping the driving rate in strain controlled simulations, it is shown by Liu et al., ${ }^{154}$ that the critical exponents of the avalanches tend towards the mean field predictions as the driving rate is increased. In contrast, for low driving rates such exponents are clearly differentiated from the predicted by Mean Field theory as can be seen in Table 2.1 (a). In the case of strong driving, the recovering of Mean Field behavior was associated to suppression of strong mechanical correlations. Besides, M. Robbins et al., and Barrat et al., analyzed the effect of inertia and damping by tuning the damping parameter $\Gamma$ in molecular dynamics simulations and elasto-plastic models respectively ${ }^{170,139}$. They demonstrated that inertia (controlled by $\Gamma$ ) has an impact in the critical exponents and promotes the emergence of events with a characteristic scale, which shows up as a hump in the power law distributions. Also in that context, T. Egami et al. showed by means of numerical analysis that avalanche behavior is promoted in fast quenched glasses compared to those obtained at lower cooling rates ${ }^{171}$. Such phenomenon was associated to the higher density of local minima on the fast quenched PEL.

The dependence of the avalanche crossover on the macroscopic rate shown in Figure 5.8 is consistent with the interpretation of the crossover given by Krisponeit et al., ${ }^{30}$ and the findings regarding driving rate effects given by Liu et al, and Salerno et al , 154,170 . In particular, avalanches in the initial regime are highly inertial due to the high strain rate, and can therefore be well modelled in the mean field approximation, whereas the second regime corresponds to an overdamped scenario of thermally activated correlated events. The shift of $t_{\text {cross }}$ towards higher times as either stress or temperature are increased can be understood in terms of the influence of both excitations on the PEL. Since temperature is a scalar quantity, its influence on the PEL consists in an isotropic reduction of all the energy barriers. On the other hand, mechanical stress induces an anisotropy in the system's exploration of the PEL, which can be understood as an effective tilt of the energy barriers. Such tilt actually reduces the barriers along a certain direction, and increase them along their opposite ones. The difference between mechanical and thermal excitation is reflected in the different functional dependence of $t_{\text {cross }}$ with stress and temperature show in Figure 5.8. The increase of low energy barriers can be associated to the promotion of flow units in the glassy matrix, that were shown to increase upon heating by Wang et al. ${ }^{172}$.

In contrast with the aforementioned strain controlled simulations ${ }^{154,139,170}$ the crossover in the creep experiments under discussion is not produced by a change on the driving rate. In the case of a stress-controlled creep experiments no external strain rate is imposed. In contrast, the sample is subjected to a constant load during the whole experiment. Thus, the change of strain rate characterized by $t_{\dot{\varepsilon}}$ should be a consequence of an internal process of the material. Such slowing down of the dynamics can be understood as an exhaustion of the low energy barriers in the PEL. At early stages of deformation, the system explores the PEL by hopping over the lowest energy barriers. Only after those low barriers have been all overcome, that regime is saturated and the system explores inherent states separated by higher effective energies, which corresponds to the second avalanching regime. The fact that $t_{\text {cross }}$ increases both as the stress and temperature 
increase is in good agreement with the previous interpretation in terms of the low energy barriers of the PEL.

Finally, the evolution of $t_{\text {cross }}$ as a function of temperature can be used to estimate an activation energy for the crossover assuming an Arrhenius relationship between $t_{\text {cross }}$ and temperature: $t_{\text {cross }}=t_{o} e^{\left(-\frac{E_{A}}{K_{B} T}\right)}$. Figure 6.1 shows the evolution of the natural logarithm of $t_{\text {cross }}$ as a function of $\frac{T_{g}}{T}$. The evolution of $\log \left(t_{\text {cross }}\right)$ with respect to $\frac{\mathrm{T}_{\mathrm{g}}}{\mathrm{T}}$ suggests that the data can be divided between a low and high temperature regime corresponding to $\frac{T}{T_{g}}<0.9$ and $\frac{T}{T_{g}}>0.9$ respectively

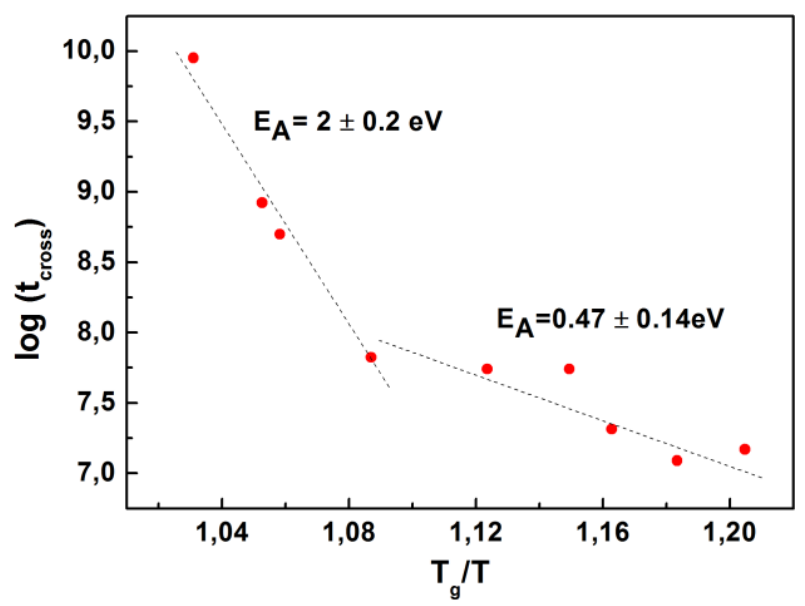

Figure 6.1 Natural logarithm of $t_{\text {cross }}$ as a function of the $\frac{\mathrm{T}_{\mathrm{g}}}{\mathrm{T}}$ plotted in an Angell plot fashion. Dashed lines represent Least Squares fits calculated in the two regimes $\frac{\mathrm{T}}{\mathrm{T}_{\mathrm{g}}}<0.9$ and $\frac{\mathrm{T}}{\mathrm{T}_{\mathrm{g}}}>0.9$.

The activation Energy calculated at low temperatures is $E_{A}\left(\frac{\mathrm{T}}{\mathrm{T}_{\mathrm{g}}}<0.9\right)=0.47 \pm 0.14 \mathrm{eV}$, whereas $E_{A}\left(\frac{T}{T_{g}}>0.9\right)=2 \pm 0.2 \mathrm{eV}$. The activation energy for the crossover at lower temperatures is in good agreement with the value for activation energy measured by Schwabe et al. for damping in $\mathrm{Pd}_{77.5} \mathrm{Cu}_{6} \mathrm{Si}_{16.5}$ at temperatures $\frac{\mathrm{T}}{\mathrm{T}_{\mathrm{g}}}<0.9,\left(E_{A} \approx 0.41 \mathrm{eV}\right)^{71}$ and the activation energy for the $\beta$-process found by for the same system by Hachenberg et al. $\left(E_{A} \approx 0.67 \mathrm{eV}\right){ }^{173}$. Such good match suggests that these three independent experiments describe the same underlying mechanism, and that avalanches might be due to the interaction of $\beta$-like events. On contrast, the higher activation energy found at temperatures approaching $\mathrm{T}_{\mathrm{g}}$ seems to indicate an onset of activation of correlated processes ( $\alpha$-processes), which could be related to avalanches involving a higher number of STZ's. 


\subsubsection{The influence of stress and temperature on the waiting time distribution shape}

Besides the shift of the crossover that was discussed in the previous section, stress and temperature affect the waiting time power law distribution in different ways. Figure 6.1 (a) shows the waiting times power law distribution of the second regime $\left(t>t_{\text {cross }}\right)$ corresponding to creep measurements performed at $\sigma=8 \mathrm{MPa}$ and several temperatures in the range $\frac{T}{T_{g}}=0.85-0.94$ replotted from Figure 5.5. It can be seen that the exponential cut-off shifts to lower waiting times as the temperature is increased, and analogous results are found comparing experiments under different applied stresses. Intuitively, such shift can be understood as a consequence of the effective decrease of the energy barriers as the temperature is increased. Such effect make longer waiting times less likely to occur as temperature is raised.

Figure 6.2(b) displays the waiting times distribution of the second regime, corresponding to creep measurements performed under several mechanical stresses at $\frac{T}{T_{g}}=0.89$, replotted as well from Figure 5.5. Figure 6.2(b) shows the development of a plateau or small hump previous to the exponential cut-off of the distributions as the mechanical stress is increased. The distortion of power law scaling was associated by Eurich et al ${ }^{142}$ to the changes in cooperativity of the system. Thus, the slight distortion of the power-law shape shown in Figure 6.2 (b) might be due to the stressinduced correlations.
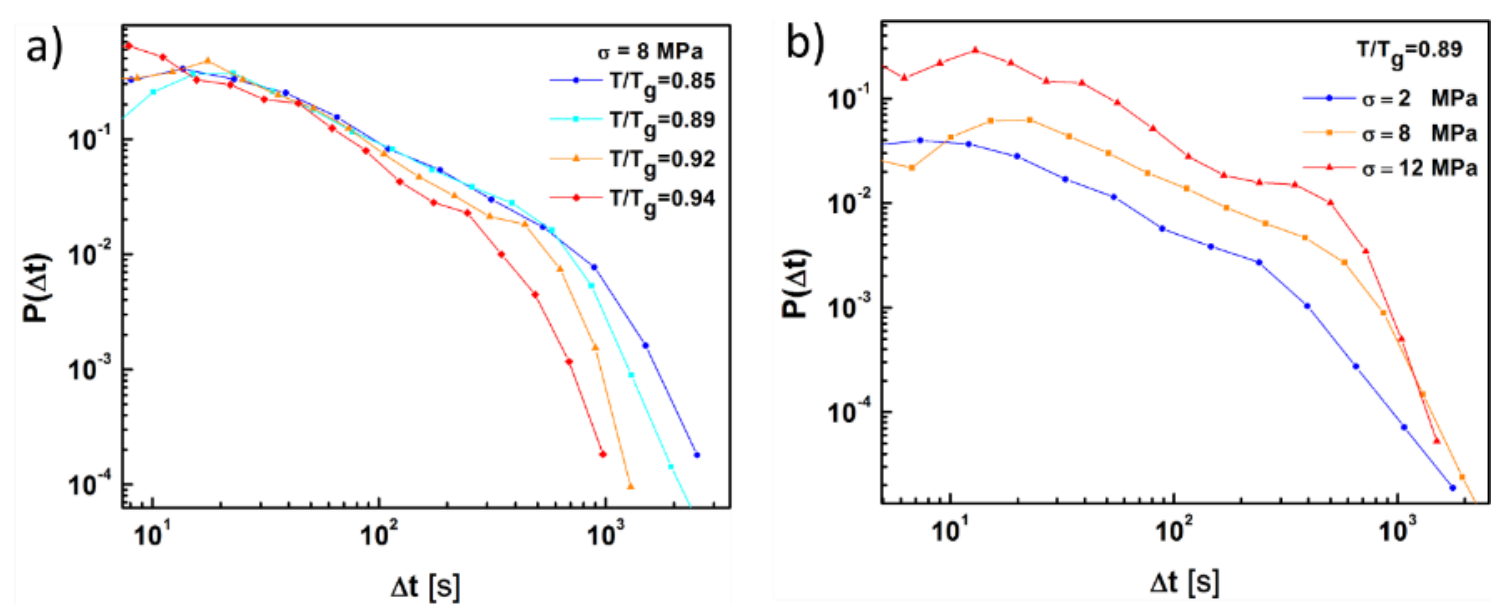

Figure 6.2 (a) Waiting time distribution yield upon creep measurements performed at $\sigma=8 \mathrm{MPa}$ and $\mathrm{T} / \mathrm{T}_{\mathrm{g}}=0.85,0.89,0.92$ and 0.94 . (b) Waiting time distribution obtained from creep measurements performed at $\mathrm{T} / \mathrm{T}_{\mathrm{g}}=0.89$ and $\sigma=$ 2, 8 and $12 \mathrm{MPa}$ 


\subsubsection{Physical interpretation of the waiting times}

Due to limitation in the apparatus spatial resolution, the fine structure of a given waiting time cannot be resolved in terms of avalanche durations $\Delta t_{\mathrm{AV}}$ and inter-events times $\Delta t_{\mathrm{it}}$, as was discussed in section 4.1.1 and schematically shown in Figure 4.2. For this reason, the interpretation of the waiting times in terms of classical avalanche parameters, such as size, duration, or interevent time has remained elusive.

However, under suitable hypotheses it is possible to associate a given waiting time $\Delta t$ to typical avalanche magnitudes. More precisely, in the hypothetical case of high avalanche activity, it could be assumed that inter-events times are negligible. Instead, many avalanches would be being triggered through the sample and as a result there would be an almost continuous overlap of events. If such were the case, it could be inferred that Equation 4.1 would simplify as follows:

$$
\Delta t=\sum_{i} \Delta t_{A V_{i}} \sum_{j} \Delta t_{i t_{j}} \sim \sum_{i} \Delta t_{A V_{i}}
$$

In that situation, in which only avalanche durations contribute to the waiting time $\Delta t$, one could define, through a coarse grain transformation ${ }^{174}$, an effective avalanche of $15 \mathrm{~nm}$ and the waiting time would be equivalent to the duration of such avalanche $\Delta t \rightarrow \Delta t_{A V}$.

On the other hand, if a regime in which avalanches are scarce is assumed, the contribution from inter-event times would be dominant within a given waiting time. Moreover, even if some avalanches take place, their total duration must be negligible compared to the total quiet or interevent time. In such scenario, Equation 4.1 could be approximated by the following expression:

$$
\Delta t=\sum_{i} \Delta t_{A V_{i}} \sum_{j} \Delta t_{i t_{j}} \sim \sum_{j} \Delta t_{i t_{j}}
$$

In that case, a given waiting time would be mainly composed by the superposition of several interevent times, and $\Delta t \rightarrow \Delta t_{i t}$.

The critical exponent predicted by mean field theories for avalanches duration scaling $P\left(\Delta T_{A V}\right) \sim \Delta T_{A V}{ }^{-\tau}$ is $\tau=-2{ }^{31}$, whereas elasto-plastic models predict $\tau=-1.4^{24}$. On the other hand it was found that inter-event times in earthquake activity exhibit a crossover from a power law scaling with exponent $\tau=-2.4$ to $\tau=-0.9^{136}$. Besides, as pointed out by Salje et al ${ }^{145} \mathrm{a}$ measured exponent may be the consequence of several underlying processes and therefore some exponent mixing is expected in real experiments. In this context, measured exponents before the crossover $\tau_{1} \sim-1.5$ are compatible with a superposition of mean field avalanches and few interevent times in which avalanches are dominant. The scaling shown in the second regime $\tau_{2} \sim-0.8$ is much closer to the measured earthquakes inter-event times, which suggest that avalanches are scarcer in such regime. The approximations illustrated in Equations (6.1),(6.2) are then compatible 
with the interpretation given by Krisponeit et $\mathrm{al}^{30}$ of a transition from mean field stress-driven plastic events to the thermal assisted collective activation of nano-shear bands. 


\subsection{Creep deformation in magnetic metallic glasses}

\subsubsection{Characterization of sample properties}

Creep experiments performed in magnetic metallic glasses were carried out in Fe-rich 2605SA1 ribbons from Metglas Inc. exhibiting positive magnetostriction with saturation magnetostriction $\lambda_{S}=27 \times 10^{-6} 167,175$.

The structure of the ribbons was checked before and after the creep measurements by XRD scans. Figure 5.13 shows diffraction patterns under different loading conditions, and it can be appreciated that the structure remained amorphous in every case. EDX spectrum reveals the composition of the sample which is $80 \% \mathrm{Fe}$. It must be noted that light atoms cannot be accurately detected by dispersed X-rays. However a small percentage of Boron is expected according to the manufacturer technical bulleting ${ }^{167}$.

Samples have been magnetically characterized by means of VSM measurements, which yield a Curie temperature $T_{c}=672 \mathrm{~K}$ in good agreement with literature and Metglas Inc. technical bulletin ${ }^{167}$. Most of the creep measurements were performed at $\mathrm{T}=553 \mathrm{~K}$, a temperature high enough to observe substantial creep deformation at the available stress range, but yet remaining in the ferromagnetic phase, which was checked by means of VSM measurements with oven mode shown in Figure 5.12 (b). Young modulus $\mathrm{E}$ was estimated from the slope of stress-strain measurements performed at room temperature to be $\mathrm{E}=105 \pm 10 \mathrm{GPa}$, in good agreement with Metglas technical bulleting specification ${ }^{167}$.

DSC scans provided a precise measure for the crystallization and Curie temperature in good agreement with the company technical data, but no clear signature of the glass transition could be appreciated. Therefore, $\mathrm{T}_{\mathrm{g}}$ was estimated by means of temperature scans under different uniaxial stresses in the DMA. This way, $\mathrm{T}_{\mathrm{g}}$ is estimated as the temperature in which the slope $\frac{\partial \varepsilon}{\partial \mathrm{T}}$ significantly deviates from linear behavior. The glass transition temperature was approximated by the temperature at which $\frac{\partial \varepsilon}{\partial \mathrm{T}}$ deviated from linearity subjected to the lowest mechanical stress $\mathrm{T}_{\mathrm{g}} \sim \mathrm{T}_{\mathrm{g}}(\sigma=2 \mathrm{MPa})=685 \pm 5 \mathrm{~K}$. 


\subsubsection{The influence of stress and temperature on creep deformation}

Figures 5.15 and 5.19 show the existence of a crossover in the distribution of waiting times yield during creep measurements of 2605SA1 under different conditions of stress and temperature. The exponents of the waiting times corresponding to the first $\left(t<t_{\text {cross }}\right)$ and second $\left(t>t_{\text {cross }}\right)$ regimes oscillate around values compatible with $\tau_{1}=-1.5, \tau_{2}=-0.8$ within error bars, in analogy with the results found in $\mathrm{Pd}_{77.5} \mathrm{Cu}_{6} \mathrm{Si}_{16.5}$. Moreover, figures 5.16 and 5.20 (a) show that $t_{\text {cross }}$ increases as both stress and temperature increase, but exhibits a different functional dependence with each of them as it was the case in $\mathrm{Pd}_{77.5} \mathrm{Cu}_{6} \mathrm{Si}_{16.5}$ experiments.

Additionally, figures 5.16 and Figure 5.20 (a) demonstrate the correlation between the avalanche regime and strain rate by showing the similar evolution of $t_{\text {cross }}$ and $t_{\dot{\varepsilon}}$ with temperature and stress respectively. There is a qualitative match between both figures of merit, which is also quantitative in the case of the stress dependence shown in Figure 5.20 (a). The aforementioned results suggest that the strain-rate dependent crossover in the creep deformation regime discussed in section 6.1 is not a specific feature of $\mathrm{Pd}_{77.5} \mathrm{Cu}_{6} \mathrm{Si}_{16.5}$ but is rather a common feature of metallic glasses.

In addition, the dispersion of effective energy barriers quantified by $W$ provides an estimation of the heterogeneity of the PEL, which has been associated to the fragility of the system ${ }^{72}$, enthalpy storage and mechanical softening ${ }^{67}$. The ability of the experimental parameter $W$ to depict the fragile-to-strong transition upon mechanical yielding shown in simulations ${ }^{72}$ is discussed in Appendix B.

Figures $5.17(\mathrm{~d})$ and $5.20(\mathrm{~b})$ show the evolution of $W$ with temperature and stress for constant stress and temperature respectively. It can be seen that $W$ increases as both temperature and stress are ramped with analogous functional dependence as $t_{\text {cross }}$ and $t_{\dot{\varepsilon}}$. This fact suggests a correlation between the avalanche regime and the dynamical heterogeneities, being the duration of the first avalanching regime proportional to the heterogeneity degree or fragility.

Remarkably, values of $t_{\text {cross }}$ in the case of Fe-rich 2605SA1, are roughly two orders of magnitude smaller than for $\mathrm{Pd}_{77.5} \mathrm{Cu}_{6} \mathrm{Si}_{16.5}$, measured in similar conditions in terms of $\frac{\sigma}{E}, \frac{\mathrm{T}}{\mathrm{T}}$. Taking into account that the first avalanche regime was associated in Section 6.1.2 to the overcome of low energy barriers, such result suggests that the PEL of the Fe-rich alloy has smaller density of low energy minima. That possibility is further supported by DSC scans of both alloys. $\operatorname{Pd}_{77.5} \mathrm{Cu}_{6} \mathrm{Si}_{16.5}$ samples exhibit a clear enthalpy hump previous to crystallization in contrast with the Fe-rich alloy. Since enthalpy storage has been associated to elastic heterogeneities and shallow minima in the $\mathrm{PEL}^{67}$, the comparison between calorimetric and avalanche dynamics experiments supports the picture of the initial avalanche regime being enhanced by elastic heterogeneities. 


\subsubsection{The influence of the magnetic field on creep deformation}

In order to interpret the influence of the magnetic field on the mechanical deformation during creep tests, the impact of both mechanic and magnetic driving on the magnetic domain structure and domain wall motion should be taken into account. Due to the preparation technique and shape, as-cast 2605SA ribbons exhibit a longitudinal anisotropy $K_{u}$ which results in a pattern of longitudinal domains along the length axes ${ }^{176}$. In the case of an alloy with positive magnetostriction, such anisotropy is further increased when a stress is applied along the ribbon length axes ${ }^{103}$. In this scenario, a magnetic field applied along any other direction would induce a rotation of the domains with an angle which results from a balance between the anisotropy and the magnetostatic energy in the minimization of the free energy. Such interplay between the anisotropy and bias field can be quantified by an anisotropy field $H_{A}$, which represents the field required to saturate the sample along a certain direction:

$$
\sin (\alpha)=\frac{H}{H_{A}}\left(H \leq H_{A}\right)
$$

where $\alpha$ represents the angle of magnetization rotation with respect to the longitudinal direction and $H$ the applied field. A detailed analysis of the magnetic domain configuration and its dependence on the mechanical stress and magnetic field in creep and stress-strain experiments is given in Appendix C. Following the results shown in there, the anisotropy field is given by:

$$
H_{A \sigma}=\frac{2 K_{u}+3 \lambda_{s} \sigma}{M_{s}}
$$

where $K_{u}$ represents the longitudinal anisotropy, $\lambda_{S}$ the saturation magnetostriction, $M_{S}$ the saturation magnetization and $\sigma$ the mechanical stress.

Subsection 5.2.5 focuses on the analysis of the influence of the intensity of magnetic field applied along the width direction $(\theta=0, \phi=0)$ on the creep deformation measured at $\frac{T}{T_{g}}=0.8$ under two different mechanical loads $\sigma=15,25 \mathrm{MPa}$. As shown in Equation (6.4), the anisotropy field increases with the stress for $\lambda_{S}>0$ alloys. It then follows that domain wall rotation angles $\alpha$ induced during creep measurements at $\sigma=15 \mathrm{MPa}$ should be larger than those achieved at $\sigma=$ $25 \mathrm{MPa}$.

The measurements done at $\sigma=15 \mathrm{MPa}$ yield a maximum of the three figures of merit $t_{\text {cross }}, t_{\dot{\varepsilon}}, W$ corresponding to an applied field $H=350$ Oe. The evolution of the three figures of merit as a function of field intensity are replotted from figures 5.2.3 and 5.2.6 in figure 6.2 for the reader convenience. Such result suggests the existence of a given domain wall orientation $\alpha_{c}$ in which the inertial avalanche regime and dynamic heterogeneities are maximized. In contrast, measurements performed at higher stress $\sigma=25 \mathrm{MPa}$ show no maximum of the three figures of merit, but instead a monotonic increase of all three as a function of field intensity. Such behavior in turn suggests that 
the range of field intensities is not enough to achieve the preferred domain orientation $\alpha_{c}$ at $\sigma=$ $25 \mathrm{MPa}$.
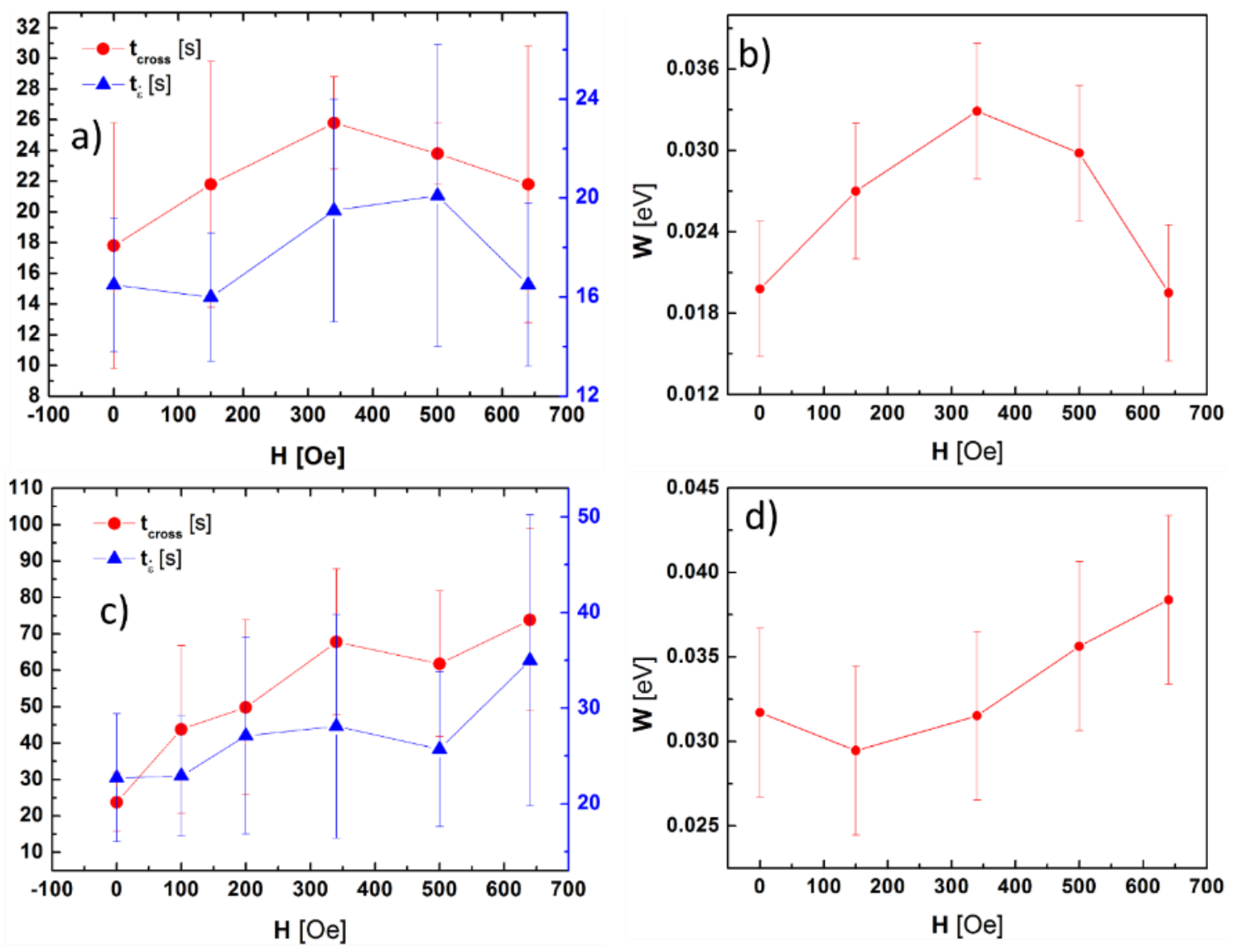

Figure 6.3 (a) Evolution of $t_{\text {cross }}$ and $t_{\dot{\varepsilon}}$ as a function of magnetic field intensity calculated from creep measurements of 2605SA1 measured at $\sigma=15 \mathrm{MPa}, \frac{\mathrm{T}}{\mathrm{T}_{\mathrm{g}}}=$ 0.8. (b) Evolution of $W$ as a function of magnetic field intensity calculated from creep measurements of 2605SA1 measured at $\sigma=15 \mathrm{MPa}, \frac{\mathrm{T}}{\mathrm{T}_{\mathrm{g}}}=0.8$. (c) Evolution of $t_{\text {cross }}$ and $t_{\dot{\varepsilon}}$ as a function of magnetic field intensity calculated from creep measurements of 2605SA1 measured at $\sigma=25 \mathrm{MPa}, \frac{\mathrm{T}}{\mathrm{T}_{\mathrm{g}}}=0.8$. (d) Evolution of $W$ as a function of magnetic field intensity calculated from creep measurements of 2605SA1 measured at $\sigma=25 \mathrm{MPa}, \frac{\mathrm{T}}{\mathrm{T}_{\mathrm{g}}}=0.8$.

The analysis of the influence of the azimuthal angle $\theta$ on the creep deformation for a constant field intensity shown in Figure 5.30 reveals a decrease of the magnitude of the three figures of merit as the angle $\theta$ is increased. An increase of $\theta$ implies a rotation of the applied field towards the out-ofplane orientation. As the bias field moves towards an out-of-plane orientation, the susceptibility dramatically decreases as shown in the hysteresis loop in Figure 5.12 and so does the magnetic field influence on the domain structure. Therefore, the effect of increasing $\theta$ is the recovering of the longitudinal arrangement of magnetic domains.

In subsection 5.26 the influence of the polar angle $\phi$ on the creep deformation was analyzed. Despite some variability on the data, a decrease of $t_{\text {cross }}$ can be seen as the magnetic field is aligned 
towards the longitudinal direction $(\phi=-90,90)$. Remarkably, maximum values of $t_{\text {cross }}$ are obtained at angles in the surroundings of $\phi=-45,45$. Such maxima are especially sharp in the case of $W$ in which two peaks at $\phi=-45,+60$ degrees can be clearly seen. The evolution of $t_{\text {cross }}, t_{\dot{\varepsilon}}$ and $W$ as function of the angle $\phi$ is replotted from Figure 5.32 in Figure 6.3 for the reader convenience.
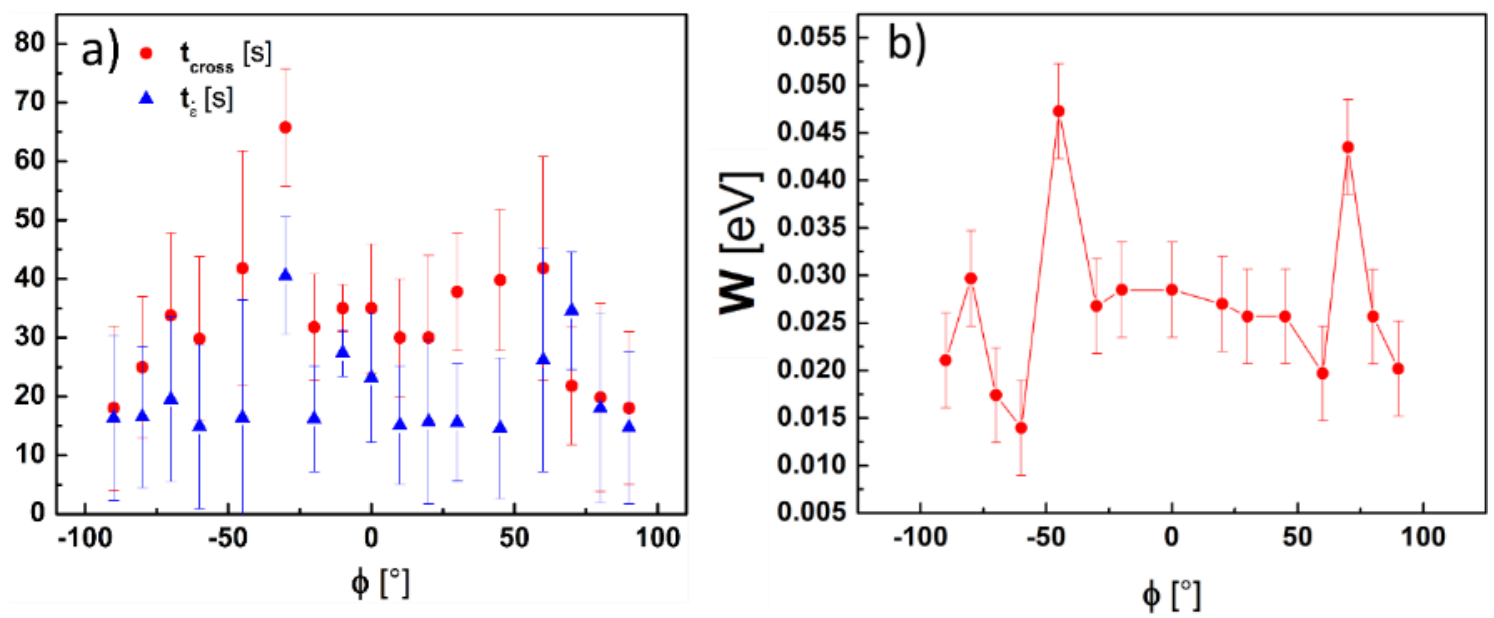

Figure 6.4 (a) Dependence of $t_{\text {cross }}$ and $t_{\dot{\varepsilon}}$ with the angle $\phi$ for creep experiments performed on 2605SA1 at $\frac{\mathrm{T}}{\mathrm{T}_{\mathrm{g}}}=0.8, \sigma=15 \mathrm{MPa}|\vec{H}|=120 \mathrm{Oe}$. (b) $W$ as a function of the angle $\phi$ for creep experiments performed on 2605SA $\frac{\mathrm{T}}{\mathrm{T}_{\mathrm{g}}}=0.8, \sigma=15 \mathrm{MPa}|\vec{H}|=120$ 0e.

The influence of both $|\vec{H}|$ and $\phi$ on the avalanches $\left(t_{\text {cross }}\right)$ and heterogeneities $(W)$ reveals the complexity of the magneto-mechanical coupling. In particular, the non-monotonic dependence of both $t_{\text {cross }}$ and $W$ with respect to $|\vec{H}|$ and $\phi$ suggests a non-linear coupling between the magnetic and mechanical subsystems and the existence of a certain domain wall orientation $\alpha_{\mathrm{c}}$ which maximizes the initial avalanche regime and the heterogeneities degree. Furthermore, Figure 6.3 (b) reveals a symmetric behavior of $W(\phi)$ which implies that is the direction of domain walls with respect to the tensile stress, and not their orientation, what more substantially affects the avalanche regime.

The coupling between the avalanche regime and the magnetization can be interpreted in terms of an interplay between Shear Transformation Zones and domain walls through magneto-elastic coupling. Under tensile loading, STZs are known to preferentially align along a roughly $45^{\circ}$ with respect to the maximum stress component due to their Eshelby field interaction ${ }^{23,177}$. On the other hand, as discussed in Chapter 2, magnetostriction is a local phenomenon, which influences the local environment inducing a local anisotropy around the magnetic atoms ${ }^{106,107}$. If domain walls are oriented parallel to the STZ preferential alignment direction ( $\pm 45^{\circ}$ under tensile loading), cascades of STZs can be triggered while keeping stress and magnetic field parallel along the domain wall. Thus, such an arrangement would be the most favorable for the STZ operation, which is in agreement with the experimental results. A schematic description of such orientation of domain walls with respect to STZs is illustrated in Figure 6.5. 


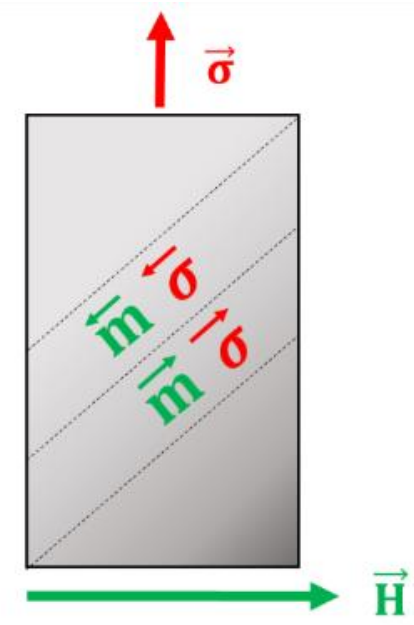

Figure 6.5 Schematic illustration of the relative orientation of the STZ stress field $(\vec{\sigma})$ and the magnetization vector $(\overrightarrow{\mathrm{m}})$ in a domain wall oriented $45^{\circ}$ respect the longitudinal axis. Dashed lines represent domain walls and red and green arrows indicate the STZ stress field and magnetization vector respectively. 


\subsection{Magnetoelastic coupling in stress-strain measurements}

In addition to creep experiments, the effect of the magnetomechanical coupling in the deformation has been studied in stress-strain tests. The motivation for these experiments is double. On the one hand stress-strain tests allows to measure deformation at room temperature, a regime in which creep is negligible. Since magnetic order is inversely proportional to temperature, magnetomechanical effects are expected to be maximized in such regime. On the other hand, in contrast to creep experiments, during stress controlled stress-strain tests the stress is constantly increased at a given stress rate $\dot{\sigma}$. Taking into account Equation 6.4 , that means that the effective anisotropy is constantly modified through the magnetostriction term $3 \lambda_{S} \sigma$, which translates into magnetization rotation during the experiment. Thus, stress-strain tests provide the opportunity to analyze the effect of domain rotations on the mechanical properties. In order to analyze the role played by the magnetostriction, all the experiments were performed on both Fe-rich 2605SA1 ${ }^{167}$ and Co-rich $\mathrm{Fe}_{2} \mathrm{Co}_{73} \mathrm{Si}_{10} \mathrm{~B}_{15}$ ribbons, exhibiting the former positive magnetostriction $\lambda_{S}=27 \times 10^{-6}$ and the latter vanishing magnetostriction in its unstressed state $\mathrm{e}^{109,108}$.

Figure 5.36 displays the global effect of the magnetic field on the mechanical behavior by means of the analysis of the macroscopic Young Modulus $E$ of both samples under different applied magnetic fields. In both alloys, E does not vary significantly as a consequence of a magnetic field oriented along the out-of-plane direction $(\theta=90)$. Such small influence is expected from the shape of the hysteresis loops shown in Figures 5.12 (a) and 5.32 which show that both alloys undergo negligible magnetization along the out of plane direction for the available field intensity range.

On the other hand, the impact of a magnetic field oriented along the width of the ribbon $(\theta=0)$ on the mechanical response of the samples can be interpreted in terms of the rotation of the magnetic domains of a magnetostrictive material, which give rise to the so called $\Delta E$-effect ${ }^{110}$. More precisely, as domains rotate, they exert an extra strain whose sign depends on the sign of the magnetostriction $\lambda_{S}$, which may induce a significant change in $E$. Such effect has been typically described by the addition of an extra strain contribution as depicted by equation 6.5 , shown below ${ }^{14}$ :

$$
E=\frac{\sigma}{\varepsilon_{e}+\varepsilon_{\lambda}}
$$

Where $\varepsilon_{e}$ represents the normal elastic strain and $\varepsilon_{\lambda}$ the magnetostrictive strain arising from the domains. As discussed in the previous subsection and Appendix $C$, the domain orientation is a result of a balance between the anisotropy and the applied field, which is described by equation 6.3. However, in the case of a stress-strain experiment, the effective anisotropy $2 K_{u}+3 \lambda_{s} \sigma$ changes continuously due to the imposed stress rate $\dot{\sigma}$. That change of stress induces a magnetization rotation during the experiment, in contrast with the static domain configuration that takes place during creep measurements. 
The evolution of the Young modulus as a function of the applied field in the Fe-rich alloy can be understood as follows: for small fields $(H<200)$ no significant rotation is induced in the magnetic domains, which remain parallel to the length orientation, and therefore the elastic modulus remains unaffected and similar to literature values ${ }^{167}$. As a field of moderate intensity (200<H>900 Oe) is applied, the magnetization initially rotates by a given angle $\alpha$. The sample magnetization rotates back throughout the experiment as the stress is increased and so does the anisotropy term $\left(2 K_{u}+3 \lambda_{s} \sigma\right)$. Through that rotation, the magnetic domains contribute with an extra magnetostrictive strain $\varepsilon_{\lambda}$ which reduces the effective quasi-static Young modulus as shown in equation (6.5). Once a certain stress $\sigma_{K}$ is applied, whose value increase with $H$, the magnetization is fully returned to the longitudinal direction and the Young modulus recovers its demagnetized value. Finally, in the case of the highest magnetic field ( $H=900 \mathrm{Oe})$ no decrease of $E$ can be seen. Such result can be associated to a fully saturation of the sample along the width axes and the incapability of the applied stress to induce any rotation.

Co-rich alloys exhibit vanishing $\lambda_{S}$ in their unstressed state, but are known to increase their magnetostriction modulus towards negative values under uniaxial stresses ${ }^{178}$. This is due to the fact that both one ion and two ion components of magnetostriction exhibit different values of their stress derivatives. Therefore although they compensate each other in the unstrained state that compensantion does not hold under stress ${ }^{178}$. That means that these alloys can be expected to exhibit a small negative magnetostriction during the stress-strain test. Hence, the anisotropy term represented by Equation (6.3) is reduced as the stress increases, which implies that the magnetic domains tend to rotate from the longitudinal towards the perpendicular direction as stress increases. Through such rotation, the magnitude of the negative strain exerted by the domains along the longitudinal direction is reduced, and therefore the Young modulus is reduced as well. In contrast to $\lambda_{S}>0$ alloys, if a magnetic field is applied perpendicular to the stress, the magnetic and the magnetostrictive energy contributions do not compete with each other and both tend to align the magnetization along the direction perpendicular to the stress. Thus, $E$ would be reduced as long as neither the stress nor magnetic field are high enough to saturate the magnetization along the perpendicular orientation with respect to the tensile stress.

The data shown in Figure 5.46 (a) are in good agreement with the interpretation given above. It can be seen therein that the macroscopic Young modulus of the Co-rich sample increases when a field $H>200$ Oe is applied along the direction $\theta=0$. That condition corresponds to the field required to saturate the magnetization along the width axes. In the remaining cases, it can be concluded that domain rotation reduces $E$ by providing a contribution that vanishes as soon as a field sufficiently intense to saturate the magnetization is applied, which turns out to be $H>200$ in this case.

The effect of the magnetic field can also be analyzed from the point of view of the fine structure of the data. Figure 5.37 shows the distribution of the normalized modulus $\hat{E}=\frac{E_{\text {macro }}}{E_{e f f}(t)}$. It is important to highlight that the definition of $E_{\text {eff }}(t)$ given in Chapter 4 , do not consist in linearizing the stressstrain curve and claim that the actual deformation behavior of the sample is purely elastic at any given time. Instead, $E_{e f f}(t)$ is defined according to the fine structure of the data. The effective modulus includes the contribution of all the elastic, anelastic and plastic deformation events that may occur in the vicinity of a time $t$ and cannot be told apart due to limits in the actual experimental resolution. Those elastic and plastic events would contribute to the storage and loss modulus, if measured with an oscillatory force and with a resolution high enough to accurately keep track of small phase angles between strain and stress ${ }^{179}$. 
As mentioned in Chapter 4, the value of $\widehat{E}$ is proportional to the strain carried out by the avalanches when $\hat{E}>1$. Due to technical constrains the minimum detectable modulus, for the size of the studied samples, is roughly $E_{\text {eff }} \sim 5 \mathrm{GPa}$. This limitation implies that the maximum measurable values of the normalized modulus are restricted to $\hat{E} \sim 10^{2}$, which hinders the assertion of precise functional patterns in the data. In addition, since the experiments were performed within the socalled elastic regime, only few, small-size avalanches are expected to occur, leading to limited statistics that also contribute to the narrow range of scaling, which can be appreciated in Figure 5.37 .

The analysis of the dependence on the magnetic field of the effective modulus reveals a difference between the Fe-rich and Co-rich alloys. On Figure 5.37(a) it can be observed a very good collapse of the $P(\hat{E})$ for all the experimental conditions in the Co-rich alloy. The data show a peak centered in $\hat{E}=1$ which corresponds to an effective modulus that matches the global macroscopic one, a feature to be expected in the linear region. The symmetric width around the peak can be associated to heterogeneities in the mechanical properties, but also to the contribution of some inevitable noise in the measurement. Finally, the asymmetric tail at values $\hat{E}>1$ can be associated to the occurrence of few strain bursts or avalanche processes. It should be highlighted that the collapse holds for all the experimental conditions in this alloy, including those that shown a significant change of $E$. This fact suggests that the superposition principle between mechanical and magnetic energy holds for all the intensities and orientations of magnetic field in this alloy with vanishing magnetostriction.

Figure 5.37(b) represents the data for the Fe-rich alloy, which shows a remarkable difference when compared to Figure $5.37(\mathrm{a})$. Although the vast majority of the data can be collapsed in a curve similar to the previous one, few data clearly deviates from such collapse. Non-collapsing data correspond with those experimental conditions in which a drop of the macroscopic modulus take place. In such cases the probability distribution of $\hat{E}$ separates from the symmetric shape and shows a small region of roughly power law decay, which can be appreciated in Figure 5.37 (b) inset. Whilst the limited accessible range of $\hat{E}$ prevents robust affirmation about a power law distribution and its specific exponent, it is clear that the magnetic field causes the collapse breakdown. Physically, this implies that the magnetic and mechanical energies are added in a non-linear way through the magnetoelastic coupling. Moreover, in those cases the probability of $\hat{E}>1$ is much higher than for the rest of the collapsed data, a fact which reflects an enhancement of avalanching activity. Those events are not uniformly distributed over the whole range of applied stresses. It can be seen in Figure 5.38 that stress-strain curves of the Fe-rich sample measured under the condition (200<H>900 0e) exhibit two different slopes separated by a kink located at $\sigma=\sigma_{K}$. The analysis of the distribution of effective modulus separated in two regimes $\sigma\left\langle\sigma_{K}\right.$ and $\sigma>\sigma_{K}$, shown in Figure 5.38 (b), reveals a predominance of smaller effective modulus $E_{\text {eff }}$, and therefore of higher renormalized modulus $\widehat{E}$, in the initial regime $\sigma<\sigma_{K}$. As previously argued, the first regime is characterized by the rotation of the magnetostrictive domains towards the stress direction. Hence, it can be concluded that mechanical avalanches are promoted by the rotation of the magnetic walls.

In conclusion, it has been shown that the magnetization affects the instantaneous mechanical response in magnetostrictive metallic glasses, by increasing the number of big avalanches as the magnetic walls rotate towards the applied stress orientation. In those cases, there is a breakdown of the collapse and the data seems to show a power law -like decay. It is worth to highlight that a linear softening of the sample would not have any impact of the $\hat{E}$ distribution. Therefore, the 
breakdown of the collapse can be associated to a non-linear addition of the magnetic energy on top of the mechanical energy ${ }^{168}$. 


\section{Conclusions and outlook}

\subsection{Conclusions}

The scope of this work is the study of deformation processes in metallic glasses by means of a statistical analysis of the intermittency in the deformation signal. In particular, this work can be divided into three parts: i) Study of avalanches produced during creep deformation of $\mathrm{Pd}_{77.5} \mathrm{Cu}_{6} \mathrm{Si}_{16.5}$ under different mechanical stresses and temperatures, ii) Analysis of avalanches during creep deformation of magnetostrictive Fe-rich 2605SA1 ribbons under magnetic driving, and iii) Deformation study of Fe-rich and Co-rich alloys subjected to tensile stress-strains tests under different magnetic excitation. The main results obtained on such issues are shortly summarized below.

Firstly, the analysis of a broad set of creep tests performed on $\mathrm{Pd}_{77.5} \mathrm{Cu}_{6} \mathrm{Si}_{16.5}$ ribbons allows to conclude that the crossover observed by Krisponeit et al. ${ }^{30}$ takes place over a wide range of stresses and temperatures. Moreover, comparison with the average strain rate suggests an intimate relation between avalanche regimes and strain rate ${ }^{35}$. The initial avalanche regime would be governed by fast triggering of STZs cascades which would produce inertial avalanches. Upon exhaustion of the first regime, a second stage develops associated to the formation of 2D correlated nano-shear bands. Several theoretical works ${ }^{154,141}$ point out that mean field descriptions successfully described avalanches under high rate driving, or in an underdamped state, whereas that approach fails as soon as inertia becomes negligible. According to such view, interactions among STZs in the first regime could be better described by a mean field approach, whereas 2D nano-shear bands after the crossover would not be consistent with a mean field approximation. Finally, the slowing down of the dynamics can be associated to a thorough exploration of the Potential Energy Landscape (PEL). More precisely, the system overcomes low energy barriers at the beginning of the creep experiment, which results in fast deformation rates. Higher energy barriers are explored only when the lower ones have been exhausted, thus yielding lower deformation rates.

A crossover in the waiting times statistics is also observed in creep tests performed on a magnetostrictive Fe-rich metallic glass. Similar exponents and dependencies of the crossover with respect to the stress and temperature as those found for $\mathrm{Pd}_{77.5} \mathrm{Cu}_{6} \mathrm{Si}_{16.5}$ point towards a universal transition in metallic glasses exposed to creep deformation. In this sense, the shorter inertial regime in the Fe-rich alloy compared with $\mathrm{Pd}_{77.5} \mathrm{Cu}_{6} \mathrm{Si}_{16.5}$ can be attributed to a lower density of minima in the Fe-alloy PEL. Experiments combining stress, temperature, and magnetic driving yield 
evidence of the influence of the magnetic excitation on the avalanche dynamics. In particular, experiments with varying intensity and polar angle $\phi$ suggest the enhancement of the mean field avalanching regime and dynamic heterogeneities in the PEL by a certain domain wall orientation $\alpha_{C}$. Although experimental constraints hamper precise estimates of $\alpha_{C}$, the results obtained are compatible with the value $\alpha_{C} \sim 45^{\circ}$ with respect to the applied uniaxial stress. Such experimental result can be interpreted in terms of a magnetically induced anisotropic modification of local yield stresses around magnetic atoms. Such anisotropy would facilitate the STZ triggering along a certain axis determined by the magnetization. Thus, the condition to maximize the coupling between the stress field generated by an STZ and the magnetization is accomplished if domain walls are oriented parallel to the STZ preferential alignment $\left(\sim \pm 45^{\circ}\right.$ under tensile loading). In that scenario, the stress field generated by cascades of STZ's is parallel to the magnetization along the domain wall.

The last part of this memoir focuses on the analysis of stress-strain tests of Fe-rich and Co-rich magnetic alloys under magnetic driving. In contrast with creep experiments, rotation of domains is expected to occur during the tests due to the imposed stress rate $\dot{\sigma}$. In this case, the change in the macroscopic Young modulus as a function of the applied field can be understood as a consequence of rotations of the domain walls of magnetostrictive magnetic domains. In this context, the Fe-rich magnetic alloy is known to exhibit positive high magnetostriction, whereas the Co-alloy exhibits small negative magnetostriction under uniaxial stress. Analysis of the data fine structure reveals a difference between the behavior of both alloys. On the one hand, the collapse of the normalized modulus $\hat{E}$ for every experimental condition in the Co-rich alloy suggests that a superposition principle between magnetic and mechanic energies holds in all cases. On the other hand, a promotion of periods of high activity associated to $\widehat{E}>1$ is observed in the Fe-rich alloy under the application of certain magnetic fields which induce domain rotations. As a consequence, the distribution of normalized modulus departs from the collapsed shape through the addition of a power law tail. Such fact reveals the non-linear addition of magnetic and mechanical energies.

In conclusion, this work provides experimental evidence of the existence of a crossover on the waiting time distribution during creep deformation in several glassy systems. Such crossover is associated to the average strain rate and is determined by the density of shallow minima in the PEL. Furthermore, the influence of magnetic excitation on avalanche dynamics is studied in two magnetic glasses under two different protocols. Analysis of creep measurements and stress-strain tests under magnetic fields allows to distinguish two different contributions of the magnetomechanical coupling. First, creep measurements permit to investigate the dependence of the orientation of domain walls with respect to the applied stress. The results in this regard hint at the existence of a certain domain wall orientation $\alpha_{C} \sim 45^{\circ}$ which maximizes the impact on the deformation. Second, stress-strain tests allow to study the influence of wall rotations on the mechanical response. Such process promotes bursts of deformation which show up as a breakdown of the normalized modulus collapse. To conclude, both analyses reveal the complexity of the magneto-mechanical coupling, in which non-linear and orientational effects appear to play a big role. 


\subsection{Outlook}

The experimental results discussed in this memoir show the complex mechanisms governing the magneto-mechanical coupling, which include non-linear and orientational effects. These results call for further experiments to provide a deeper insight into the microscopical origin of such coupling. Some personal suggestions for future research pathways are listed below.

As discussed in section 6.2 the orientation of magnetic domain walls with respect to the applied stress seems to play an important role in the magnetomechanical effect on the mechanical avalanches. Therefore, it would be of great interest to measure avalanches on samples with engineered domain patterns and orientation. Domain tailoring in metallic glasses can be performed by means of thermal annealing under stress and magnetic fields ${ }^{180,181,182}$. Thus, the role played by domain wall orientation could be accurately analyzed by means of appropriately treated samples.

Another interesting fact that deserves further attention is the local effect of magnetostriction on the mechanical environment of magnetic atoms and its influence on the STZ operation. Given the difficulty of resolving atomic positions in deformation experiments on metallic glasses, Molecular Dynamics simulations might assist in this regard. On the other hand, both single STZs and percolation of STZs ensembles were successfully observed by confocal microscopy in deformation experiments on colloidal glasses ${ }^{87}$. The influence of magnetic fields on local shearing events could be monitored if similar experiments could be done involving magnetically-coated spheres under the application of an external bias field, for example, by means of an electromagnet. Such experiments would allow for direct observation of the effect of a magnetic field on STZ interactions.

Also, time scales of magnetomechanical effects that were discussed in terms of rotation and orientation of magnetic walls might be analyzed by means of ultrasound measurements of Young modulus under magnetic driving. In contrast with the experiments shown through his memoir, ultrasound experiments, at high enough frequencies, only probe fast processes. Such experiments may help to separate the influence of the magnetomechanical coupling in slow $(\alpha)$ and fast $(\beta)$ relaxations.

More generally, to achieve a better understanding of the magnetomechanical coupling effect on avalanches, it would be advisable to perform deformation experiments with higher resolution. Although waiting time analysis has demonstrated its utility for the description of avalanching processes, the limited DMA-7 resolution constrains the intermittency to be described by a single parameter (i.e., the waiting time duration). In contrast, more sensitive methods like acoustic emission measurements ${ }^{147,183}$ could characterize avalanches with more detail, thus permitting to quantify sizes, duration and shape profiles. Such complete description would allow for avalanche characterization beyond power law exponents, which is believed to yield a more complete picture of the underlying process ${ }^{132}$. Besides, acoustic emission experiments can be done using several transducers placed at different sample locations. That would allow to investigate the localization of avalanches.

Finally, although mechanical and magnetic avalanche events have been shown to be simultaneously triggered in numerical simulations ${ }^{155}$, a synchronous analysis of both mechanical and magnetic phenomena has never been experimentally carried out. Such experimental set-up would be technologically challenging, given the difficulty to reach high accuracy on both mechanical and 
magnetic measurements at the same time. However, if a Kerr-effect microscope and a high precision DMA could be combined without resolution loss in each device, it should be possible to monitor deformation avalanches, Barkhausen noise and domain structure at the same time. It is likely that such experiment would significantly improve the understanding of magneto-mechanical coupling in metallic glasses and its influence on the underlying avalanche dynamics. 


\section{Acknowledgments}

First and foremost, I want to thank Professor Dr. Konrad Samwer for giving me the chance to carry out my doctoral thesis under his supervision. During this time, he has generously provided me with his ideas and advice, and has always been available to share his expertise in glass physics during individual and group meetings. Additionally, he has supported my participation in many international conferences and workshops, which served me greatly and helped me continue to gain scientific knowledge and develop my presentation skills. Specially, he encouraged me to test my own ideas and develop independent work, which I consider a key skill for my professional development. Finally, I also appreciate his support and advice regarding my future career plans. Joining the glass group in Göttingen has also been a very valuable experience beyond science and thesis work, resulting in my appreciation of the German language and culture, science and ski conferences in Switzerland, meeting new friends in the vibrant town of Göttingen, and every day lessons of a life far away from home and my comfort zone. Vielen Dank!

Furthermore, I want to thank Professor Dr. Cynthia Volkert for being my second supervisor and for her interesting suggestions and remarks. Professor Dr. Vasile Mosneaga, Professor Dr. Michael Seibt, Dr. Claus Heussinger and Dr. Richard Vink are also thankfully acknowledged for accepting to take part of my thesis committee.

As part of the Marie Curie ITN "VitriMetTech" I had the opportunity to learn, interact and collaborate with several scientists of different European institutions. I am therefore very grateful to the European Union for financial support. I am also especially thankful to Professor Dr. Georgios Evangelakis for hosting me at the University of loannina and sharing his knowledge in glass and computational physics through several workshops and discussions. I have also been invited by Dr. Beatrice Ruta to work together with Martin Lüttich in Synchrotron experiments in ESRF in Grenoble, a valuable experience that I am also very thankful for.

Pursuing my PhD at the $1^{\text {st }}$ Physikalisches Institut has been a pleasant experience due to the nice atmosphere created by the people working there. I would like to thank all members and former members for their friendliness and willingness to help. I want to thank the help from all the technical staff: The help and patience of Uta Filippich teaching me how to operate the DMA and helping to solve any experimental or technical problem that I have encountered. Additionally she has been a great ski instructor. I also greatly appreciate the support of Carsten Mahn with every computer-related issue, Simon Sloldt support with EDX measurements and the printing of this thesis, the guidance of Kathrin Gerkhe regarding X-ray Diffractometry and everyday lab-related issues, and the assistance of Kai Dörner and Dennis Köln with gas bottles handling. To Dennis Köln I must also acknowledge his support in the repair of the gas sensor cell of the DMA glovebox and 
his help with sample cutting. I also want to thank Harmut Eichenberg and the staff of the $1^{\text {st }}$ Institute workshop and central workshop for their availability and help in designing experimental pieces.

I want to thank Dr. Manuel Mchalwat for his help with countless experimental issues. His unfortunate office location in front of the mechanics lab made him an easy victim of my many "kurze Fragen" which often were not so short, but nevertheless he was always ready to help. To Martin Lüttich I am thankful for his hospitality in Grenoble during our joint synchrotron sessions at ESRF. I am very thankful to Dr. Stefan Küchemann, who is always available for scientific questions and discussions even after leaving the group. Additionally, I have learned a lot from our scientific discussions with Dr. Birte Riechers, who was always available to help me in many aspects, including being extremely patient during the presentation rehearsals for my german courses. I want to thank Victor Pfahl who, despite not working exactly on glasses, joined me in many brainstorming sessions. Many projects, including one of the magnet holder designs, were carried out with his help. I also appreciate his friendship and his willingness to keep joining me to table soccer breaks despite his clear negative score balance. Finally, I want to thank Dr. Alexandra Lagogianni, who really did make a difference when she joined us in the office. Beyond her vast help in scientific aspects, she made the time spent in the office much more fun and became a truly close friend.

I also would like to thank all the friends and people who shared time with me during my PhD time also outside the university, including flatmates, spanish and latin community and football colleagues. I will keep the memories of all the gatherings, trips, parties, and football games, (specially our $10^{\text {th }}$ Position in Uni-Liga with Atlético Chingones). I am sure that this work could not have been done without the necessary support that they provided me when needed. I would also like to mention all friends that I left in Spain, who kept feeling very close regardless of the distance.

Finally, I want to thank my parents Miguel and Pilar, my sister Elena and my girlfriend Diana. Being far away from all of them only helped me realize how important they are to me. I appreciate very much their advice and unconditional support, which I especially needed and felt during the hardest times. All of them in their different ways have always been and continue to be a role model for me. i Muchísimas gracias por todo! 


\section{Appendix A. Influence of parameters $\alpha_{1}, \alpha_{2}$ on $t_{\dot{\varepsilon}}$}

The procedure to define the crossover according to the average strain rate was introduced in Section 4.1.2. $t_{\dot{\varepsilon}}$ is computed as the experimental time which fulfils the condition (4.1), which is rewritten below:

$$
t_{\dot{\varepsilon}}=\min (t) \quad \text { such that } \quad \varepsilon(t)<\alpha \varepsilon_{\text {steady-state }}
$$

Making use of condition (A.1) with two parameters: $\alpha_{1}, \alpha_{2}$ two experimental times are obtained $t_{\alpha_{1}}, t_{\alpha_{2}}$. The crossover time $t_{\dot{\varepsilon}}$ is then calculated as the average of $t_{\alpha_{1}}$ and $t_{\alpha_{2}}$, and the upper and lower limits of the error bar are estimated from $t_{\alpha_{1}}$ and $t_{\alpha_{2}}$ respectively.

The results shown in this memoir are calculated using the values $\alpha_{1}=20, \alpha_{2}=10$. This appendix is devoted to the study of the influence of the parameters $\alpha_{1}, \alpha_{2}$ on the estimation of $t_{\dot{\varepsilon}}$. Figure A.1 below shows the crossover time $t_{\dot{\varepsilon}}$ calculated from the creep measurements of 2605SA1 at constant temperatures and several stresses making use three different sets of parameters: $\left(\alpha_{1}=10, \alpha_{2}=5\right),\left(\alpha_{1}=20, \alpha_{2}=10\right)$ and $\left(\alpha_{1}=40, \alpha_{2}=20\right)$.
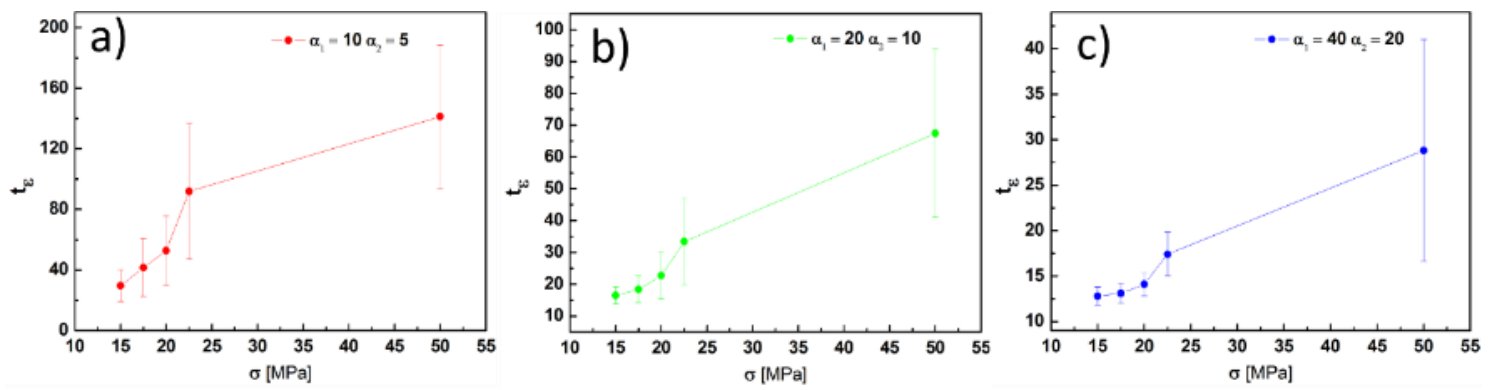

Figure A.1 (a) Evolution of $t_{\dot{\varepsilon}}$ as function of stress calculated from creep measurements of 2605SA1 at $\frac{T}{T_{g}}=0.8$, calculated using $\left(\alpha_{1}=10, \alpha_{2}=5\right)$. (b) Evolution of $t_{\dot{\varepsilon}}$ as function of stress calculated from creep measurements of 2605SA1 at $\frac{T}{T_{g}}=0.8$, calculated using $\left(\alpha_{1}=20, \alpha_{2}=10\right)$. (c) Evolution of $t_{\dot{\varepsilon}}$ as function of stress calculated from creep measurements of 2605SA1 at $\frac{T}{T_{g}}=0.8$, calculated using ( $\left.\alpha_{1}=40, \alpha_{2}=20\right)$

Figures A.1 (a), (b) and (c) show the evolution of $t_{\dot{\varepsilon}}$ as a function of stress calculated using the parameters $\left(\alpha_{1}=10, \alpha_{2}=5\right),\left(\alpha_{1}=20, \alpha_{2}=10\right)$ and $\left(\alpha_{1}=40, \alpha_{2}=20\right)$ respectively. Since 
parameters $\alpha_{1}, \alpha_{2}$ define the threshold for the fulfillment of condition (4.1), $t_{\dot{\varepsilon}}$ values decrease as $\alpha_{1}, \alpha_{2}$ increase. However, it can be seen that qualitatively the functional dependence of $t_{\dot{\varepsilon}}$ with stress does not depend on the choice of $\alpha_{1}, \alpha_{2}$. Such independence is highlighted in Figure A.2, which shows the evolution of normalized values of $t_{\dot{\varepsilon}}$ calculated using the three different sets of $\left(\alpha_{1}, \alpha_{2}\right)$ as a function of stress and temperature. From Figure A.2 it can be inferred that, although the specific value of $t_{\dot{\varepsilon}}$ depends on the choice of $\left(\alpha_{1}, \alpha_{2}\right)$, the qualitative functional dependence of $t_{\dot{\varepsilon}}$ remains invariant under different thresholds.
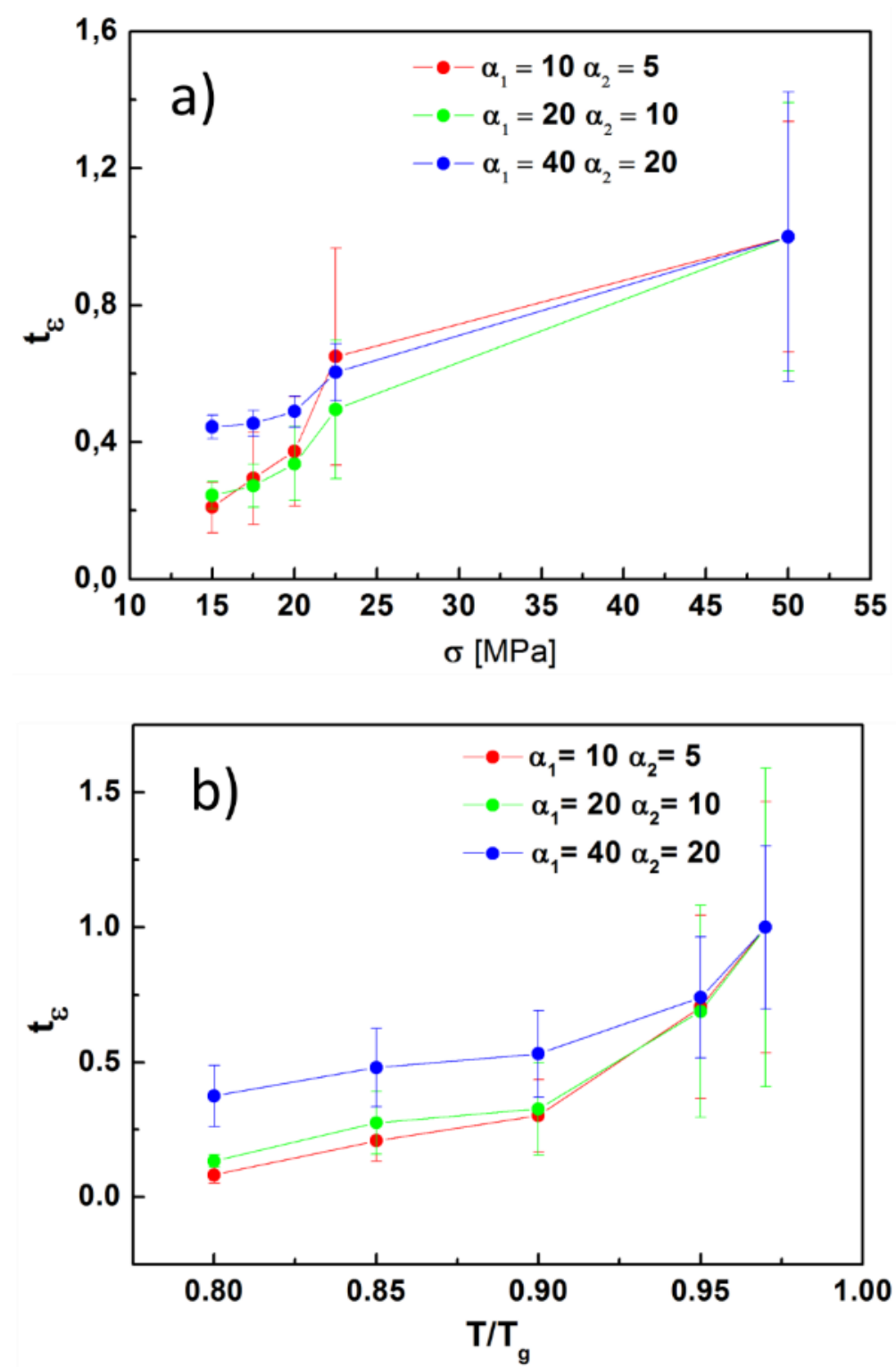

Figure A.2 (a) evolution of normalized $t_{\dot{\varepsilon}}$ as function of stress calculated from creep measurements of 2605SA1 at $\frac{T}{T_{g}}=0.8$, for different thresholds $\left(\alpha_{1}=10, \alpha_{2}=\right.$ 5), $\left(\alpha_{1}=20, \alpha_{2}=10\right)$ and $\left(\alpha_{1}=40, \alpha_{2}=20\right)$. (b) Evolution of normalized $t_{\dot{\varepsilon}}$ as function of temperature calculated from creep measurements of 2605SA1 at stress $\sigma=15 \mathrm{MPa}$ for different thresholds $\left(\alpha_{1}=10, \alpha_{2}=5\right),\left(\alpha_{1}=20, \alpha_{2}=\right.$ $10)$ and $\left(\alpha_{1}=40, \alpha_{2}=20\right)$ 


\section{Appendix B. Evaluation of $W$ as fragility estimator}

The experiments described in this appendix were performed in order to test the fitness of parameter $W$ defined in Chapter 4 , as an estimator of the heterogeneities in the PEL. The analysis presented herein attempts to check if the strain induced fragility transition observed by Yu et al., in Molecular Dynamics simulations ${ }^{72}$ could be monitored measuring $W$ in creep experiments.

As discussed in section 6.2, $\mathrm{T}_{\mathrm{g}}$ in Fe-rich 2605SA1 glasses was estimated from the deviation of the slope $\frac{\partial \varepsilon}{\partial T}$ from linear behavior in temperature scans under uniaxial tensile mechanical stress, being the yielding temperature estimated as a function of the applied stress $\sigma$. The analysis described in this appendix was performed on creep experiments measured under constant normalized temperatures $\frac{\mathrm{T}}{\mathrm{T}_{\mathrm{g}}(\sigma)}$, but with different ratios between mechanical and thermal excitation i.e., yielding temperatures reached at low stresses and high temperatures or at higher stresses and lower temperatures.

The evolution of $W$ as function of the mechanical stress $\sigma$ under condition of constant $\frac{\mathrm{T}}{\mathrm{T}_{\mathrm{g}}(\sigma)}$ calculated from creep measurements in Fe-rich 2605SA1 is shown in Figure B.1. A decrease of $W$ as a function of increasing stress is observed, which is in good agreement with the finding of Yu et al., in molecular dynamics ${ }^{72}$ and reflects the difference between mechanical and thermal driving described in Chapter 2. The result shown in figure B.1 supports the applicability of $W$ as an experimental estimator of heterogeneities in the PEL throughout this memoir.

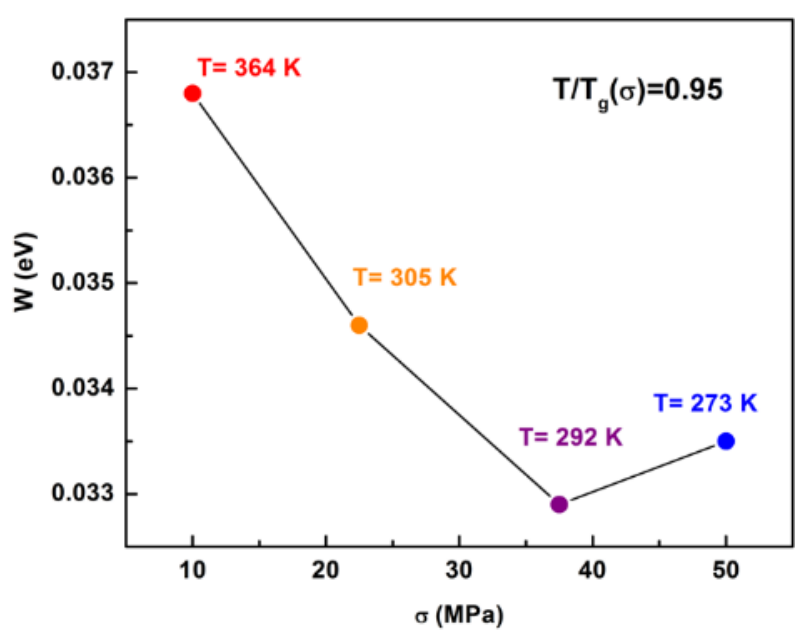

Figure B.1 Evolution of $W$ as function of mechanical stress $\sigma$ at constant $\frac{\mathrm{T}}{\mathrm{T}_{\mathrm{g}}(\sigma)}$ calculated from creep measurements of 2605SA1 


\section{Appendix C. Magnetic domains in ribbons under tensile loading}

In order to interpret the influence of the magnetic field on the mechanical deformation during creep tests, the influence of both mechanical and magnetic driving on the magnetic domain structure should be taken into account. Due to the preparation technique, as-cast ribbons in absence of external bias field exhibit a longitudinal anisotropy $K_{u}$ which results in a pattern of longitudinal domains along the length axis 176,184. A magnetic field applied longitudinally ( $\theta=0, \phi=90,-90)$ would induce the growth of those domains whose magnetization lays in the same direction as the applied field, through domain wall motion, resulting in an increase of the macroscopic magnetization $\vec{M}$. In such scenario, there would be no domain rotation, since the domains were already aligned along the magnetic field orientation.

On the contrary, a magnetic field applied perpendicular to the longitudinal axes induces a domain rotation towards the field direction. The orientation of the magnetization, is determined as a balance between the anisotropy and magnetostatic energy contributions ${ }^{14},{ }^{103}$. Both energy terms are shown in equation (C.1), in which for simplicity is assumed that the applied field lays in the ribbon plane $(\theta=0)$

$$
U=K_{u} \cos ^{2}(\gamma)-M_{s} H \cos (\gamma-\phi)
$$

Where $\gamma$ is the angle of the magnetization with respect to the ribbon width, $K_{u}$ represents the anisotropy along the length direction, $M_{S}$ the saturation magnetization and $\phi$ the angle of the bias field with respect to the width direction.

In the case of a magnetic field applied along the width direction $(\theta=0, \phi=0)$, the minimization of the energy in equation (C.1) leads to:

$$
\cos (\gamma)=\frac{M_{s} H}{2 K_{u}}=M_{s} \frac{H}{H_{A}}\left(H \leq H_{A}\right)
$$

Were $H_{A}=\frac{2 K_{u}}{M_{S}}$ denotes the anisotropy field, which represents the field required to rotate the magnetization along the width direction. In the case of a magnetostrictive material, the anisotropy would read: 


$$
H_{A \sigma}=\frac{2 K_{u}+3 \lambda_{S} \sigma}{M_{S}}
$$

Therefore, the effect of a mechanical stress on the domain rotation would depends on the sign of the magnetostriction $\lambda_{S}$. In the case of materials with $\lambda_{S}>0$, a tensile stress will hamper the domain rotation $\alpha$ produced by a magnetic field perpendicular to that stress. On the other hand, a tensile stress applied to a $\lambda_{S}<0$ alloy will promote the domain rotation, since the anisotropy field is reduced through the magnetostriction. 


\section{Literature}

1. Cheng, Y.-T. \& Johnson, W. L. Disordered Materials: A Survey of Amorphous Solids. Science (80-. ). 235, 997-1002 (1987).

2. Oppenheim, A. Leo, Bril, R.H., Barag, D., Von Saldern, A. Glass and glassmaking in ancient Mesopotamia; an edition of the cuneiform texts which contain instructions for glassmakers with a catalogue of surviving objects. (Corning Museum of Glass Inc., 1970).

3. Yu, H.-B., Luo, Y. \& Samwer, K. Ultrastable Metallic Glass. Adv. Mater. 25, 5904-5908 (2013).

4. Schlenkrich, Felix, Seyffarth, Susanne, Fuchs, Britta, Krebs, H.-U. Pulsed laser deposition of polymer-metal nanocomposites. Appl. Surf. Sci. 257, 5362-5365 (2011).

5. Johnson, W. L. Bulk Glass-Forming Metallic Alloys: Science and Technology. MRS Bull. 24, 42-56 (1999).

6. Klement, W., Willens, R. H. \& Duwez, P. Non-crystalline Structure in Solidified Gold-Silicon Alloys. Nature 187, 869-870 (1960).

7. Greer, A. L. \& Ma, E. Bulk Metallic Glasses: At the Cutting Edge of Metals Research. MRS Bull. 32, 611-619 (2007).

8. Schroers, J. Processing of Bulk Metallic Glass. Adv. Mater. 22, 1566-1597 (2010).

9. Ashby, M. \& Greer, A. Metallic glasses as structural materials. Scr. Mater. (2006).

10. Gloriant, T. Microhardness and abrasive wear resistance of metallic glasses and nanostructured composite materials. J. Non. Cryst. Solids 316, 96-103 (2003).

11. Ashby, M. F. \& Greer, A. L. Metallic glasses as structural materials. Scr. Mater. 54, 321-326 (2006).

12. Liquid Metals Technologies Inc.

13. Tiberto, P., Baricco, M., Olivetti, E. \& Piccin, R. Magnetic Properties of Bulk Metallic Glasses. Adv. Eng. Mater. 9, 468-474 (2007).

14. Coey, J. M. . Magnetism and Magnetic Materials. Cambridge 52, (2010).

15. Luborsky, F. E., Becker, J. J., Frischmann, P. G. \& Johnson, L. A. Potential of amorphous alloys for application in magnetic devices. J. Appl. Phys. 49, 1769-1774 (1978).

16. Hasegawa, R. Soft magnetic properties of metallic glasses. J. Magn. Magn. Mater. 41, 79-85 (1984).

17. Hernando, A. \& Vazquez, M. Metallic glasses and sensing applications. J. Phys. E 21, 1129- 
1139 (1988).

18. Vazquez, M. \& Hernando, A. A soft magnetic wire for sensor applications. J. Phys. D. Appl. Phys. 29, 939-949 (1996).

19. Grimes, C. A. et al. Wireless Magnetoelastic Resonance Sensors: A Critical Review. Sensors 2, 294-313 (2002).

20. Argon, A. . Plastic deformation in metallic glasses. Acta Metall. 27, 47-58 (1979).

21. Goldstein, M. Viscous Liquids and the Glass Transition: A Potential Energy Barrier Picture. J. Chem. Phys. 51, 3728-3739 (1969).

22. Johnson, W. \& Samwer, K. A universal criterion for plastic yielding of metallic glasses with a (T/T g) 2/3 temperature dependence. Phys. Rev. Lett. 95, 195501 (2005).

23. Dasgupta, R., Hentschel, H. G. E. \& Procaccia, I. Microscopic Mechanism of Shear Bands in Amorphous Solids. Phys. Rev. Lett. 109, 255502 (2012).

24. Ferrero, E., Martens, K. \& Barrat, J. Relaxation in yield stress systems through elastically interacting activated events. Phys. Rev. Lett. 113, 248301 (2014).

25. Homer, E. R., Rodney, D. \& Schuh, C. a. Kinetic Monte Carlo study of activated states and correlated shear-transformation-zone activity during the deformation of an amorphous metal. Phys. Rev. B - Condens. Matter Mater. Phys. 81, 1-11 (2010).

26. Lewandowski *, J. J., Wang, W. H. \& Greer, A. L. Intrinsic plasticity or brittleness of metallic glasses. Philos. Mag. Lett. 85, 77-87 (2005).

27. Nicolas, A., Ferrero, E. E., Martens, K. \& Barrat, J.-L. Deformation and flow of amorphous solids: a review of mesoscale elastoplastic models. arXiv:1708.09194 (2017).

28. Antonaglia, J. et al. Tuned critical avalanche scaling in bulk metallic glasses. Sci. Rep. 4, 4382 (2014)

29. Dalla Torre, F.H., Klaumünzer, D., Maas, R., Löffler, J. F. Stick-slip behavior of serrated flow during inhomogeneous deformation of bulk metallic glasses. Acta Mater. 58, 3742-3750 (2010).

30. Krisponeit, J. et al. Crossover from random three-dimensional avalanches to correlated nano shear bands in metallic glasses. Nature 5, 3616 (2014).

31. Dahmen, K., Ben-Zion, Y. \& Uhl, J. Micromechanical Model for Deformation in Solids with Universal Predictions for Stress-Strain Curves and Slip Avalanches. Phys. Rev. Lett. 102, 175501 (2009).

32. Maloney, C. E. \& Robbins, M. O. Anisotropic Power Law Strain Correlations in Sheared Amorphous 2D Solids. Phys. Rev. Lett. 102, (2009).

33. Sethna, J. P., Dahmen, K. a \& Myers, C. R. Crackling noise. Nature 410, 242-50 (2001).

34. Salje, E. K. H. \& Dahmen, K. a. Crackling Noise in Disordered Materials. Annu. Rev. Condens. Matter Phys. 5, 233-254 (2014).

35. Herrero-Gómez, C. \& Samwer, K. Stress and temperature dependence of the avalanche dynamics during creep deformation of metallic glasses. Sci. Rep. 6, 33503 (2016).

36. Turnbull, D. Under what conditions can a glass be formed? Contemp. Phys. 10, 473-488 (1969). 
37. Angell, C. A. Structural instability and relaxation in liquid and glassy phases near the fragile liquid limit. J. Non. Cryst. Solids 102, 205-221 (1988).

38. Debenedetti, P. \& Stillinger, F. Supercooled liquids and the glass transition. Nature 410, 259267 (2001).

39. Stillinger, F. H. \& Debenedetti, P. G. Glass Transition Thermodynamics and Kinetics. Annu. Rev. Condens. Matter Phys. 4, 263-285 (2013).

40. Cavagna, A. Supercooled liquids for pedestrians. Phys. Rep. 476, 51-124 (2009).

41. Brüning, R. \& Samwer, K. Glass transition on long time scales. Phys. Rev. B 46, 11318-11322 (1992).

42. Küchemann, S. \& Samwer, K. Ultrafast heating of metallic glasses reveals disordering of the amorphous structure. Acta Mater. 104, 119-124 (2016).

43. Egami, T. Atomic level stresses. Prog. Mater. Sci. 56, 637-653 (2011).

44. Angell, C. A., Ngai, K. L., McKenna, G. B., McMillan, P. F. \& Martin, S. W. Relaxation in glassforming liquids and amorphous solids. J. Appl. Phys. 88, (2000).

45. Cheng, Y. Q., Sheng, H. W. \& Ma, E. Relationship between structure, dynamics, and mechanical properties in metallic glass-forming alloys. Phys. Rev. B 78, 14207 (2008).

46. Concustell, A., Méar, F. O., Suriñach, S., Baró, M. D. \& Greer, A. L. Structural relaxation and rejuvenation in a metallic glass induced by shot-peening. Philos. Mag. Lett. 89, 831-840 (2009).

47. Ketov, S. V. et al. Rejuvenation of metallic glasses by non-affine thermal strain. Nature 524, 200-203 (2015).

48. Hufnagel, T. C. Metallic glasses: Cryogenic rejuvenation. Nat. Mater. 14, 867-868 (2015).

49. Singh, S., Ediger, M. D. \& de Pablo, J. J. Ultrastable glasses from in silico vapour deposition. Nat. Mater. 12, 139-144 (2013).

50. Lüttich, Martin, Giordano, Valentina M., Le Floch, Sylvie, Pineda, Eloi, Zontone, Federico, Luo, Yuansu, Samwer, Konrad, Ruta, B. Anti-Aging in ultrastable metallic glasses. Submitted (2017).

51. Angell, C. A. Relaxation in liquids, polymers and plastic crystals -strong/fragile patterns and problems. J. Non. Cryst. Solids 131133, 13-31 (1991).

52. Böhmer, R. \& Angell, C. A. Correlations of the nonexponentiality and state dependence of mechanical relaxations with bond connectivity in Ge-As-Se supercooled liquids. Phys. Rev. $B$ 45, 10091-10094 (1992).

53. Krausser, J., Samwer, K. H. \& Zaccone, A. Interatomic repulsion softness directly controls the fragility of supercooled metallic melts. PNAS 112, 13762-13767 (2015).

54. Lagogianni, A. E., Krausser, J., Evenson, Z., Samwer, K. \& Zaccone, A. Unifying interatomic potential, $g(r)$, elasticity, viscosity, and fragility of metallic glasses: analytical model, simulations, and experiments. J. Stat. Mech. Theory Exp. 84001 (2016).

55. Kob, W. \& Andersen, H. C. Scaling Behavior in the $\beta$-Relaxation Regime of a Supercooled Lennard-Jones Mixture. Phys. Rev. Lett. 73, 1376-1379 (1994).

56. Kob, W. \& Andersen, H. C. Testing mode-coupling theory for a supercooled binary Lennard- 
Jones mixture. II. Intermediate scattering function and dynamic susceptibility. Phys. Rev. E 52, 4134-4153 (1995).

57. Lunkenheimer, P., Schneider, U., Brand, R. \& Loid, A. Glassy dynamics. Contemp. Phys. 41, 15-36 (2000).

58. Kohlrausch, R. Theorie des elektrischen Rückstandes in der Leidener Flasche. Ann. der Phys. und Chemie 167, 179-214 (1854).

59. Ediger, M. D. Spatially Heterogeneous dynamics in supercooled liquids. Annu. Rev. Phys. Chem. 51, 99-128 (2000).

60. Richert, R. Heterogeneous dynamics in liquids: fluctuations in space and time. J. Phys. Condens. Matter 14, 201 (2002).

61. Wagner, H. et al. Local elastic properties of a metallic glass. Nat. Mater. 10, 439-442 (2011).

62. Stillinger, F. H. A Topographic View of Supercooled Liquids and Glass Formation. Science 267, 1935-1939 (1995).

63. Harmon, J. S., Demetriou, M. D., Johnson, W. L. \& Samwer, K. Anelastic to Plastic Transition in Metallic Glass-Forming Liquids. Phys. Rev. Lett. 99, 135502 (2007).

64. Demetriou, M. D. et al. Cooperative Shear Model for the Rheology of Glass-Forming Metallic Liquids. Phys. Rev. Lett. 97, 65502 (2006).

65. Derlet, P. M. \& Maaß, R. Linking high- and low-temperature plasticity in bulk metallic glasses: thermal activation, extreme value statistics and kinetic freezing. Philos. Mag. 93, 4232-4263 (2013).

66. Derlet, P. M. \& Maaß, R. Linking high- and low-temperature plasticity in bulk metallic glasses II: use of a log-normal barrier energy distribution and a mean-field description of hightemperature plasticity. Philos. Mag. 94, 2776-2803 (2014).

67. Ross, Perry, Küchemann, Stefan, Derlet, P.M., Yu, Haibin, Arnold, Walter, Liaw, Peter, Samwer, Konrad, Maas, R. Linking macroscopic rejuvenation to nano-elastic fluctuations in a metallic glass. Acta Mater. 138, 111-118 (2017).

68. Dziuba, T. Lokale mechanische Eigenschaften von ultrastabilen metallischen Gl国asernitle. (Georg-August-Universität Göttingen, 2016).

69. Guan, P., Chen, M. \& Egami, T. Stress-Temperature Scaling for Steady-State Flow in Metallic Glasses. Phys. Rev. Lett. 104, 205701 (2010).

70. Berthier, L. \& Barrat, J.-L. Shearing a Glassy Material: Numerical Tests of Nonequilibrium Mode-Coupling Approaches and Experimental Proposals. Phys. Rev. Lett. 89, 95702 (2002).

71. Schwabe, M., Bedorf, D. \& Samwer, K. Influence of stress and temperature on damping behavior of amorphous Pd77.5Cu6.0Si16.5 below Tg. Eur. Phys. J. E 34, 91 (2011).

72. Yu, H.-B., Richert, R., Maaß, R., Samwer, K. \& Chen, M. W. Strain induced fragility transition in metallic glass. Nat. Commun. 6, 7179 (2015).

73. Mayr, S. G. Activation Energy of Shear Transformation Zones: A Key for Understanding Rheology of Glasses and Liquids. Phys. Rev. Lett. 97, 195501 (2006).

74. Sliding and deformation of metallic glass: experiments and MD simulations. J. Non. Cryst. Solids 317, 206-214 (2003). 
75. Chen, H. S. Ductile-brittle transition in metallic glasses. Mater. Sci. Eng. 26, 79-82 (1976).

76. Yavari, A. R., Lewandowski, J. J. \& Eckert, J. Mechanical Properties of Bulk Metallic Glasses. MRS Bull. 635-638 (2007).

77. Spaepen, F. A microscopic mechanism for steady state inhomogeneous flow in metallic glasses. Acta Metall. 25, 407-415 (1977).

78. Steif, P.S, Spaepen, Frans, Hutchinson, J. W. Strain localization in amorphous metals. Acta Metall. 30, 447-455 (1982).

79. Maaß, R. \& Löffler, J. F. Shear-Band Dynamics in Metallic Glasses. Adv. Funct. Mater. 25, 2353-2368 (2015).

80. Torre, F. D., Klaumünzer, D., Maaß, R. \& Löffler, J. Stick-slip behavior of serrated flow during inhomogeneous deformation of bulk metallic glasses. Acta Mater. 58, 3742-3750 (2010).

81. Dubach, Alan, Dalla, Florian H, Torre, Dalla, Löffler, J. F. Constitutive model for inhomogeneous flow in bulk metallic glasses. Acta Mater. 57, 881-892 (2009).

82. Hays, C. C., Kim, C. P. \& Johnson, W. L. Microstructure Controlled Shear Band Pattern Formation and Enhanced Plasticity of Bulk Metallic Glasses Containing in situ Formed Ductile Phase Dendrite Dispersions. Phys. Rev. Lett. 84, 2901-2904 (2000).

83. Song, K.K, Pauly, S, Zhang, Y, Scudino, S, Garggarella, P, Surreddi, K.B, Kühn, U, Eckert, J. Significant tensile ductility induced by cold rolling in Cu47.5Zr47.5Al5 bulk metallic glass. Intermetallics 19, 1394-1398 (2011).

84. Schuh, C., Hufnagel, T. \& Ramamurty, U. Mechanical behavior of amorphous alloys. Acta Mater. 55, 4067-4109 (2007).

85. Ashby, M. F. A first report on deformation-mechanism maps. Acta Metall. 20, 887-897 (1972).

86. Cohen, M. H. \& Turnbull, D. Molecular Transport in Liquids and Glasses. J. Chem. Phys. 31, 1164-1169 (1959).

87. Schall, P., Weitz, D. \& Spaepen, F. Structural rearrangements that govern flow in colloidal glasses. Science (80-. ). 318, 1895-1899 (2007).

88. Dasgupta, R., Hentschel, H. \& Procaccia, I. Microscopic mechanism of shear bands in amorphous solids. Phys. Rev. Lett. 109, 255502 (2012).

89. Dasgupta, R., Karmakar, S. \& Procaccia, I. Universality of the plastic instability in strained amorphous solids. Phys. Rev. Lett. 108, 75701 (2012).

90. Martens, K., Bocquet, L. \& Barrat, J. Spontaneous formation of permanent shear bands in a mesoscopic model of flowing disordered matter. Soft Matter 8, 4197 (2012).

91. Zink, M., Samwer, K., Johnson, W. \& Mayr, S. Plastic deformation of metallic glasses: size of shear transformation zones from molecular dynamics simulations. Phys. Rev. B 73, 172203 (2006).

92. Eshelby, J. D. The Determination of the Elastic Field of an Ellipsoidal Inclusion, and Related Problems. Proc. R. Soc. A Math. Phys. Eng. Sci. 241, 376-396 (1957).

93. Gendelman, O., Jaiswal, P. K., Procaccia, I., Sen Gupta, B. \& Zylberg, J. Shear Transformation Zones: State determined or protocol dependent? EPL (Europhysics Lett. 109, 16002 (2015). 
94. Patinet, S., Vandembroucq, D. \& Falk, M. L. Connecting Local Yield Stresses with Plastic Activity in Amorphous Solids. Phys. Rev. Lett. 117, 45501 (2016).

95. Puosi, F., Rottler, J. \& Barrat, J.-L. Time-dependent elastic response to a local shear transformation in amorphous solids. Phys. Rev. E 89, 42302 (2014).

96. Dasgupta, R., Hentschel, H. \& Procaccia, I. Yield strain in shear banding amorphous solids. Phys. Rev. E 87, 22810 (2013).

97. Amorphous and nanocrystalline materials for applications as soft magnets. Prog. Mater. Sci. 44, 291-433 (1999).

98. Saini, S., Frankel, R., Stark, D. \& Ferrucci, J. Magnetism: a primer and review. Am. J. Roentgenol. 150, 735-743 (1988).

99. Egami, T. Magnetic amorphous alloys: physics and technological applications. Reports Prog. Phys. 47, 1601-1725 (1984).

100. Tiberto, P., Baricco, M., Olivetti, E. \& Piccin, R. Magnetic Properties of Bulk Metallic Glasses. Adv. Eng. Mater. 9, 468-474 (2007).

101. O'Handley, R. C. Physics of ferromagnetic amorphous alloys. J. Appl. Phys. 62, R15-R49 (1987).

102. Lachowicz, Hk, Szymcak, H. Magnetostriction of amorphous magnetic materials. J. Magn. Magn. Mater. 41, 327-334 (1984).

103. Livingston, J. D. Magnetomechanical properties of amorphous metals. Phys. Status Solidi 70, 591-596 (1982).

104. Barandiaran, J. M., Gutierreez, J., Garcia-Arribas, A., Squire, P. T. \& Atkinson, D. Magnetoelastic coupling constant of amorphous ferromagnetic alloys-A critical study. IEEE Trans. Magn. 36, 3241-3243 (2000).

105. Furthmuller, J., Fahnlet, M. \& Herzers ', G. Theory of magnetostriction in amorphous and polycrystalline ferromagnets. J. Phys. F Met. Phys 16, 255-258 (1986).

106. O'Handley, R. Magnetostriction of ferromagnetic metallic glasses. Solid State Commun. 21, 1119-1122 (1977).

107. Fähnle, M. \& Egami, T. Phenomenological theory of magnetostriction in amorphous ferromagnets. J. Appl. Phys. 53, 2319-2320 (1982).

108. Hernando, A., Madurga, V., Núñez de Villavicencio, C. \& Vázquez, M. Temperature dependence of the magnetostriction constant of nearly zero magnetostriction amorphous alloys. Appl. Phys. Lett. 45, 802-804 (1984).

109. Madurga, V., Vazquez, M., Hernando, A., Nielsen, O. V. Magnetostriction of amorphous (Co1-xFex)75Si15B10 ribbons $(0 \leqslant x \leqslant 0.12)$ and its temperature dependence. Solid State Commun. 52, 701-703 (1984).

110. Kersten, M. Über den Temperaturkoeffizienten des Elastizitätsmoduls ferromagnetischer Stoffe. Zeitschrift für Phys. 85, 708-716 (1933).

111. Herrero-Gómez, C., Marín, P. \& Hernando, a. Bias free magnetomechanical coupling on magnetic microwires for sensing applications. Appl. Phys. Lett. 103, 142414 (2013).

112. A remote query magnetostrictive viscosity sensor. Sensors Actuators A Phys. 80, 8-14 (2000). 
113. Herrero-Gómez, C. \& Aragón, A. Stress and field contactless sensor based on the scattering of electromagnetic waves by a single ferromagnetic microwire. Appl. Phys. 105, 92405 (2014).

114. Amorphous metallic glass biosensors. Intermetallics 30, 80-85 (2012).

115. Gutenberg, B, Richter, C. F. Seismicity of the earth and associated phenomena. (1954).

116. Faillettaz, J., Louchet, F. \& Grasso, J.-R. Two-Threshold Model for Scaling Laws of Noninteracting Snow Avalanches. Phys. Rev. Lett. 93, 208001 (2004).

117. Benza, V. G., Nori, F. \& Pla, O. Mean-field theory of sandpile avalanches: From the intermittent- to the continuous-flow regime. Phys. Rev. E 48, 4095-4098 (1993).

118. Buldyrev, S. V., Ferrante, J. \& Zypman, F. R. Dry friction avalanches: Experiment and theory. Phys. Rev. E 74, 66110 (2006).

119. Sethna, J. P. et al. Hysteresis and hierarchies: Dynamics of disorder-driven first-order phase transformations. Phys. Rev. Lett. 70, 3347-3350 (1993).

120. Cizeau, P., Zapperi, S., Durin, G. \& Stanley, H. Dynamics of a Ferromagnetic Domain Wall and the Barkhausen Effect. Phys. Rev. Lett. 79, 4669-4672 (1997).

121. Vives, E. et al. Distributions of avalanches in martensitic transformations. Phys. Rev. Lett. 72, 1694-1697 (1994).

122. Field, S., Witt, J., Nori, F. \& Ling, X. Superconducting Vortex Avalanches. Phys. Rev. Lett. 74, 1206-1209 (1995).

123. Politzer, P. A. Observation of Avalanchelike Phenomena in a Magnetically Confined Plasma. Phys. Rev. Lett. 84, 1192 (2000).

124. Bouchaud, J.-P. Power laws in economics and finance: some ideas from physics. Quant. Financ. 1, 105-112 (2001).

125. Rational group decision making: A random field Ising model at $\mathrm{T}=0$. Phys. A Stat. Mech. its Appl. 238, 66-80 (1997).

126. Lombardi, F., Herrmann, H. J., Perrone-Capano, C., Plenz, D. \& De Arcangelis, L. Balance between Excitation and Inhibition Controls the Temporal Organization of Neuronal Avalanches. Phys. Rev. Lett. 108, 228703 (2012).

127. Kuntz, M. C., Perkovic, O., Dahmen, K. A., Roberts, B. W. \& Sethna, J. P. Hysteresis, avalanches, and noise. Comput. Sci. Eng. 1, 73-81 (1999).

128. Bak, P., Tang, C. \& Wiesenfeld, K. Self-organized criticality: An explanation of the $1 / \mathrm{f}$ noise. Phys. Rev. Lett. 59, 381-384 (1987).

129. Bak, P., Tang, C. \& Wiesenfeld, K. Self-organized criticality. Phys. Rev. A 38, 364-374 (1988).

130. Massobrio, P., de Arcangelis, L., Pasquale, V., Jensen, H. J. \& Plenz, D. Criticality as a signature of healthy neural systems. Front. Syst. Neurosci. 9, 22 (2015).

131. Friedman, N. et al. Universal Critical Dynamics in High Resolution Neuronal Avalanche Data. Phys. Rev. Lett. 108, 208102 (2012).

132. Papanikolaou, S., Bohn, F., Sommer, R. \& Durin, G. Universality beyond power laws and the average avalanche shape. Nat. Phys. 7, 316-320 (2011).

133. Durin, G. \& Zapperi, S. Scaling exponents for barkhausen avalanches in polycrystalline and 
amorphous ferromagnets. Phys. Rev. Lett. 84, 4705-8 (2000).

134. Vespignani, A. \& Zapperi, S. How self-organized criticality works: A unified mean-field picture. Phys. Rev. E 57, 6345-6362 (1998).

135. Zapperi, S., Lauritsen, K. B. \& Stanley, H. E. Self-Organized Branching Processes: Mean-Field Theory for Avalanches. Phys. Rev. Lett. 75, 4071-4074 (1995).

136. Baró, J. et al. Statistical Similarity between the Compression of a Porous Material and Earthquakes. Phys. Rev. Lett. 110, 88702 (2013).

137. Salerno, K. \& Robbins, M. Effect of inertia on sheared disordered solids: Critical scaling of avalanches in two and three dimensions. Phys. Rev. E 88, 62206-62 (2013).

138. Vives, E., Soto-Parra, D., Mañosa, L., Romero, R. \& Planes, A. Driving-induced crossover in the avalanche criticality of martensitic transitions. Phys. Rev. B 80, 180101 (2009).

139. Karimi, K., Ferrero, E. E. \& Barrat, J.-L. Inertia and universality of avalanche statistics: The case of slowly deformed amorphous solids. Phys. Rev. E 95, 13003 (2017).

140. Salerno, K. M., Maloney, C. E. \& Robbins, M. O. Avalanches in Strained Amorphous Solids: Does Inertia Destroy Critical Behavior? Phys. Rev. Lett. 109, 105703 (2012).

141. Salerno, K. M. \& Robbins, M. O. Effect of inertia on sheared disordered solids: Critical scaling of avalanches in two and three dimensions. Phys. Rev. E 88, 62206-62 (2013).

142. Eurich, C. W., Herrmann, J. M. \& Ernst, U. A. Finite-size effects of avalanche dynamics. Phys. Rev. E 66, 66137 (2002).

143. Ryu, K.-S., Akinaga, H. \& Shin, S.-C. Tunable scaling behaviour observed in Barkhausen criticality of a ferromagnetic film. Nat. Phys. 3, 547-550 (2007).

144. Pradhan, S., Hansen, A. \& Hemmer, P. C. Crossover Behavior in Burst Avalanches: Signature of Imminent Failure. Phys. Rev. Lett. 95, 125501 (2005).

145. Salje, E. K. H., Planes, A. \& Vives, E. Analysis of crackling noise using the maximum-likelihood method: Power-law mixing and exponential damping. Phys. Rev. E 96, (2017).

146. Becker, R. \& Orowan, E. Über sprunghafte. Dehnung von Zinkkristallen. Z. Phys 79, 566-572 (1932).

147. Weiss, J. et al. From Mild to Wild Fluctuations in Crystal Plasticity. Phys. Rev. Lett. 114, 105504 (2015).

148. On the critical character of plasticity in metallic single crystals. Mater. Sci. Eng. A 424, 190195 (2006).

149. Richeton, T., Weiss, J. \& Louchet, F. Breakdown of avalanche critical behaviour in polycrystalline plasticity. Nat. Mater. 4, 465-469 (2005).

150. Richeton, Thiebaud, Weiss, Jerome, Louchet, F. Dislocation avalanches: Role of temperature, grain size and strain hardening. Acta Mater. 53, 4463-4471 (2005).

151. Antonaglia, J. et al. Bulk Metallic Glasses Deform via Slip Avalanches. Phys. Rev. Lett. 112, 155501 (2014).

152. Lagogianni, A., Liu, C., Martens, K. \& Samwer, K. Plastic avalanches in the so-called elastic regime of metallic glasses. Phys. Rev. B Submitted, (2017).

153. Budrikis, Z., Castellanos, D. F., Sandfeld, S., Zaiser, M. \& Zapperi, S. Universal features of 
amorphous plasticity. Nat. Commun. 8, (2017).

154. Liu, C., Ferrero, E., Puosi, F., Barrat, J. \& Martens, K. Driving rate dependence of avalanche statistics and shapes at the yielding transition. Phys. Rev. Lett. (2016).

155. Hentschel, H., Procaccia, I. \& Gupta, B. Anatomy of plastic events in magnetic amorphous solids. Phys. Rev. E 93, 33004 (2016).

156. Durin, G. \& Zapperi, S. Barkhausen noise in soft amorphous magnetic materials under applied stress. J. Appl. Phys. 85, 5196 (1999).

157. Bragg, W. H. \& Bragg, W. L. The Reflection of X-rays by Crystals. Proc. R. Soc. A Math. Phys. Eng. Sci. 88, 428-438 (1913).

158. Sheng, H. W., Luo, W. K., Alamgir, F. M., Bai, J. M. \& Ma, E. Atomic packing and short-tomedium-range order in metallic glasses. Nature 439, 419-425 (2006).

159. Quantum Design Inc. at <https://www.qdusa.com/>

160. Poynting, J. . On pressure perpendicular to the shear planes in finite pure shears, and on the lengthening of loaded wires when twisted. Proc. R. Soc. A, 546 (1909).

161. Weissenber, K, Freeman, S. M. Principles of Rheological Measurement. Proc. Gen. Conf. Br. Rheol. Club ,Bedford Coll. London 33-75 (1946).

162. Perkin Elmer Inc. at <http://www.perkinelmer.com/>

163. Greer, A. L. \& Spaepen, F. Creep, Diffusion, and Structural Relaxation in Metallic Glasses. Ann. N. Y. Acad. Sci. 371, 218-237 (1981).

164. Weiss, M., Moske, M. \& Samwer, K. Kohlrausch exponent of amorphous Zr 65 Al 7.5 Cu 27.5 determined by anelastic relaxation measurements. Cit. Appl. Phys. Lett 69, (1996).

165. Gong, P. et al. Review on the Research and Development of Ti-Based Bulk Metallic Glasses. Metals (Basel). 6, 264 (2016).

166. Jiang, W., Fan, G., Liu, F., ... G. W.-J. of materials \& 2006, U. Rate dependence of shear banding and serrated flows in a bulk metallic glass. J. Mater. Res. 21, 2164-2167 (2006).

167. (www.metglas.com). Metglas. Inc.

168. Herrero-Gómez, C. \& Samwer, K. Mechanical avalanches promoted by magnetoelastic coupling in magnetic metallic glasses. Submitted (2017).

169. Schwabe, M., Kuechemann, S., Wagner, H., Bedorf, D. \& Samwer, K. Activation volume of microscopic processes in amorphous Pd 77.5Cu6.0Si16.5 due to stress and temperature. $J$. Non. Cryst. Solids 357, 490-493 (2011).

170. Salerno, K. M. \& Robbins, M. O. Effect of inertia on sheared disordered solids: Critical scaling of avalanches in two and three dimensions. Phys. Rev. E 88, 62206-62 (2013).

171. Fan, Y., Iwashita, T. \& Egami, T. Crossover from Localized to Cascade Relaxations in Metallic Glasses. Phys. Rev. Lett. 115, (2015).

172. Wang, Z., Sun, B. A., Bai, H. Y. \& Wang, W. H. ARTICLE Evolution of hidden localized flow during glass-to-liquid transition in metallic glass. Nat. Commun. 5, 5823 (2014).

173. Hachenberg, J. et al. Merging of the $\alpha$ and $\beta$ relaxations and aging via the Johari-Goldstein modes in rapidly quenched metallic glasses. Appl. Phys. Lett. 92, 131911 (2008). 
174. Sethna, J., Dahmen, K. \& Myers, C. Crackling noise. Nature 410, 242-50 (2001).

175. Spano, M. L., Hathaway, K. B. \& Savage, H. T. Magnetostriction and magnetic anisotropy of field annealed Metglas* 2605 alloys via dc M-H loop measurements under stress. J. Appl. Phys. 53, 2667-2669 (1982).

176. Azuma, D., Hasegawa, R., Saito, S. \& Takahashi, M. Effect of residual strain in Fe-based amorphous alloys on field induced magnetic anisotropy and domain structure. J. Appl. Phys. 113, 17A339 (2013).

177. Zhang, Z. F., Eckert, J. \& Schultz, L. Difference in compressive and tensile fracture mechanisms of Zr59Cu20Al10Ni8Ti3 bulk metallic glass. Acta Mater. 51, 1167-1179 (2003).

178. Barandiarán, J. M. et al. Temperature, stress, and structural-relaxation dependence of the magnetostriction in ( Co 0.94 / BFe 0.06 ) 75 / BSi 15 B 10 glasses. Phys. Rev. B 35, 50665071 (1987).

179. Ni, X., Papanikolaou, S., Vajente, G. \& Adhikari, R. Probing Microplasticity in Small-Scale FCC Crystals via Dynamic Mechanical Analysis. Phys. Rev. Lett. 118, 155501 (2017).

180. Chen, H. S., Ferris, S. D., Gyorgy, E. M., Leamy, H. J. \& Sherwood, R. C. Field heat treatment of ferromagnetic metallic glasses. Appl. Phys. Lett. 26, 405-406 (1975).

181. Mbengue, S. S., Buiron, N. \& Lanfranchi, V. Macroscopic modeling of anisotropic magnetostriction and magnetization in soft ferromagnetic materials. J. Magn. Magn. Mater. 404, 74-78 (2016).

182. Brinkman, W. F. et al. Magnetic Annealing and Directional Ordering of an Amorphous Ferromagnetic Alloy. Phys. Rev. Phys, Rev. Phys. Rev. 6M. B. Brodsky, Phys. Rev. B 4, (1968).

183. Weiss, J. \& Marsan, D. Three-dimensional mapping of dislocation avalanches: clustering and space/time coupling. Science 299, 89-92 (2003).

184. Leamy, H. J. et al. Ferromagnetic domain structure of metallic glasses. Appl. Phys. Lett. 26, 259-260 (1975). 


\section{List of figures}

Figure 2.1 Temperature dependence of a liquid Volume or Entalphy at constant pressure. ${ }^{38}$ 8

Figure 2.2 Angell representation of the logarithm of glass former viscosities as a function of $\mathrm{Tg} / \mathrm{T}$. Strong glass formers show linear behavior in such scaling, whereas fragile glasses deviate from linearity 38

Figure 2.3 (a)Self-intermediate scattering function as a function of time for different temperatures calculated from simulations in Lennard-Jones glass. (b)Temperature dependence of the peak dielectric relaxation frequency of a glass.former 38

Figure 2.4 Mean-Square-Displacement (MSD) as a function of the logarithm of time in a LennardJones system ${ }^{56}$

Figure 2.5 Map of the local contact resonance frequencies of (a) PdCuSi glass and (b) (100)-SrTiO crystal ${ }^{61}$

Figure 2.6 (a) Schematic illustration of a Potential Energy Landscape of a glass. (b)Mean energy per particle of a Lennard-Jones glass as a function of temperature ${ }^{38}$.

Figure 2.7 Schematic section of a PEL, in which the anelastic reversible $\beta$-processes and the plastic irreversible $\alpha$-processes are represented. ${ }^{63}$

Figure 2.8 Schematic representation of the Potential Energy Landscape of a strong (a) and fragile (b) glass..$^{38}$

Figure 2.9 Two dimensional plot of the viscosity as a function of normalized stress and temperature calculated by means of molecular dynamics simulations ${ }^{69}$

Figure 2.10 Elastic limit and Young modulus for more than 1500 metals, alloys, composites and metallic glasses ${ }^{9}$.

Figure 2.11 Stress-Temperature deformation map for amorphous materials ${ }^{85}$ 
Figure 2.12 Shear rate-Temperature deformation map for amorphous materials 85

Figure 2.13 Shear Transformation Zone observed in a sheared colloidal glass by means of confocal microscopy ${ }^{88}$

Figure 2.14 Stress-temperature map of the STZ cooperativity calculated from kinetic Montecarlo simulations ${ }^{25}$

Figure 2.15 Triangular Map of magnetostriction values as a function of the element composition for $\mathrm{Fe}, \mathrm{Co}$ and $\mathrm{Ni}$ alloy.The solid line indicates the composition with vanishing magnetostriction ${ }^{14} \ldots 27$

Figure 2.16 Young modulus as a function of magnetic field intensity for a metallic glass with magnetic domains parallel $(E \|)$ and perpendicular $(E \perp)$ to the applied bias field ${ }^{14}$

Figure 2.17 Probability distribution of avalanche sizes in Barkhausen Noise experiments for amorphous and crystalline samples ${ }^{134}$

Figure 2.18 (a) Effect of the cooperativity parameter $\alpha$ on the avalanche size probability distribution in a stick-slip model ${ }^{143}$. (b) Inertial effects on the probability distribution of avalanche sizes calculated from an elasto-plastic model. The events are divided in small power law distributed avalanches (light yellow) and big inertial avalanches with a characteristic length scale ${ }^{140}$

Figure 2.19 Waiting time probability distribution measured during a creep test on PdCuSi at $\sigma=15$ $\mathrm{MPa}$ and $\mathrm{T}=593 \mathrm{~K}$. The distribution is split into two regimes with different slopes, originating from the first $100 \mathrm{~min}$ of the creep curve and the subsequent part.

Figure 2.20 (a) Barkhausen noise signal of a magnetic metallic glass for different values of mechanical stress. (b) Probability distribution of Avalanche size (Power of the voltage peaks) for different values of applied stress. The inset shows how cut-off shifts towards higher avalanche sizes as the mechanical stress increases ${ }^{157}$ 35

Figure 3.1. (a) XRD spectra of As-cast $P d_{77.5} \mathrm{Cu}_{6} S i_{16.5}$ measured with $\mathrm{Cu} \mathrm{K} \alpha$ radiation. (b) XRD spectra of As-cast 2605SA1 measured with a Mo K $\alpha$ source.

Figure 3.2 (a) Schematic representation of a typical DSC protocol. It is very important that both positive temperature ramps are measured in the same conditions, since the data resulting from them will be subtracted (b) Example of a DSC measurement carried on an As-cast $P d_{77.5} \mathrm{Cu}_{6} \mathrm{Si}_{16.5}$ measured at heating rates of $20 \mathrm{~K} / \mathrm{min}$. Tg and Tx can be estimated from the heat flow peak and sharp minimum respectively.

Figure 3.3 Main components of the PPMS equipped with the VSM option from Quantum Design: Linear motor (top) and coil set (bottom) ${ }^{160}$. 
Figure 3.4 (a) Hysteresis loop measured along the width direction of $2605 S A 1$ ribbon. (b) $M(T)$ vs $T$ curve of the same ribbon measured with a magnetic field of 300 Oe.

Figure 3.5 (a) Picture of the DMA and glovebox set up in the Mechanical Analysis Laboratory of the $1^{\text {st }}$ Institute of Physics in Göttingen. (b) Sketch of the different components of the glovebox-DMA set-up.

Figure 3.7 (a) Schematic representation of a creep-recovery protocol. (b) Schematic creep curve, reproduced from ${ }^{164}$.

Figure 3.8 (a) Sketch of a stress-controlled stress-strain protocol. (b) Experimental stress-strain curves of Ti-based Bulk metallic glasses measured in tension and compression respectively ${ }^{166} \ldots . .50$

Figure 3.9 Magnetic Dynamic Mechanical Analyzer

Figure 3.10. Calibration of permanent magnets: $\mathrm{H}$ vs $d$ curves for the three rectangular magnets measured with a Hall probe at room temperature.

Figure 3.11 Experimental stress-strain curves of $P d_{77.5} \mathrm{Cu}_{6} S i_{16.5}$ samples measured at room temperature at zero field (black line) and at $\mathrm{H}=950$ Oe (red line)

Figure 4.1 (a) Tensile creep deformation of a $P d_{77.5} C u_{6} S i_{16.5}$ ribbon subjected to a stress of $\sigma=$ $25 \mathrm{MPa}$ and temperature $T / \mathrm{Tg}=0.95$ during two hours. (b) Zoom of the creep deformation in the interval $t=(16790-16850)$ seconds, which reveals a staircase -like fine structure of horizontal jumps separated by $15 \mathrm{~nm}$.

Figure 4.2 (a) Plot of a waiting time(blue line) and two deformation trajectories (red lines) that would give rise to that waiting time.L: length, t: time. (b) Representation of the main components of an inttermittent deformation process: i) Avalanches: characterized by their size, duration and shape and ii) inter-event times. The avalanches have been plotted with a symetrical shape for simplicity.

Figure 4.3 Illustration of the filtering method. The corresponding algorithm compares the value of the functions $P(t), q(t)$ between each pair of horizontal steps. The values of $P(t), q(t)$ are shown for each time step, and the transition or jump is defined by the shortest time that fullfils $P(t)>$ $q(t)$. 
Figure 4.4. Comparison between linear and logarithmic binning. (a) Linear representation of the waiting time distribution $P(\Delta t)$ obtained from a Fe-rich SA2605 ribbon measured at $\sigma=15 \mathrm{MPa}$ and TTg $=0.85$, calculated with linear binning(red) and logarithmic binning (blue). (b) logarithmic scale representation of the waiting time distribution $P(\Delta t)$ obtained from the same experiment and calculated with linear binning(red) and logarithmic binning (blue) 60

Figure 4.5 (a) Creep curve of a $P d_{77.5} C u_{6} S i_{16.5}$ subjected to a tensile stress of $\sigma=25 \mathrm{MPa} T T g=$ 0.95 . The crossover is shown by the colored background and takes place at tcross $=1.64 \pm$ $0.6104 \mathrm{~s}$. (b) Waiting time distribution before the crossover $P(\Delta t) t<t i$, after the crossover $P(\Delta t) t>t i$ and for the whole experimental time. 62

Figure 4.6 Evolution of the strain, strain rate, and second derivative of the strain as a function of the experimental time in a creep experiment performed in a $P d_{77.5} \mathrm{Cu}_{6} S i_{16.5}$ ribbon subjected to a tensile stress of $\sigma=25 \mathrm{MPa}$ and a temperature of $\mathrm{T} / \mathrm{Tg}=0.95$. The creep signal has been smoothed, applying a moving average filter with a window size of 100 seconds, in order to remove the fine structure of the data so that time derivatives could actually be computed. Inset: zoom of strain rate and second derivative of the strain at early times of the creep measurement. 63

Figure 4.7. Strain rate as a function of time for a $P d_{77.5} C u_{6} S i_{16.5}$ ribbon subjected to a tensile stress of $\sigma=25 \mathrm{MPa}$ and a temperature of $T / \mathrm{Tg}=0.95$. the crossover in strain rate $t \varepsilon$ is shown as well as its lower and higher bounds $t \alpha 1$ and $t \alpha 2$.

Figure 4.8 . Energy dispersion analysis of a creep curve. (a) Creep curve of a Fe-rich 2605SA1 measured at stress $15 \mathrm{MPa}$ and temperature $T / \mathrm{Tg}=0.95$.(b) scatter plot of the Energy barrier as a function of the experimental time. (c) Evolution of the Energy residuals, using a non-parametric LOESS fit on the scatter plot. (d) Probability distribution function of the distribution of residuals $P(\Delta E)$, the FWHM provides a quantitative measure of the dispersion in the scatter plot. 66

Figure 4.9 (a) strain versus stress measured in a stress ramp conducted on an Fe-rich 2605SA1 ribbon at $\mathrm{T}=30{ }^{\circ} \mathrm{C}$ with a stress rate of $1.25 \times 10-2 \mathrm{MPa} / \mathrm{s}$ from $\sigma=10-30 \mathrm{MPa}$. The inset reveals a fine structure of the data, which motivates the definition of the effective modulus. (b) scatter plot representing the evolution of the effective modulus with respect to the applied stress for the stress-strain curve shown in Figure 4.9.a......

Figure 4.10 Probability distribution function of the effective modulus measured from a stress-strain experiment of Fe-rich $2605 \mathrm{SA} 1$ ribbon at $\mathrm{T}=30{ }^{\circ} \mathrm{C}$ with a stress rate of $1.25 \times 10-2 \mathrm{MPa} / \mathrm{s}$ from $\sigma=10-30 \mathrm{MPa}$.

Figure 4.11 Probability distribution of the renormalized modulus $P(E)$ calculated from a stressstrain experiment of Fe-rich $2605 \mathrm{SA} 1$ ribbon at $\mathrm{T}=300 \mathrm{~K}$ with a stress rate of $1.25 \times 10-2 \mathrm{MPa} / \mathrm{s}$ from $\sigma=10-30 \mathrm{MPa}$. 
Figure $5.2 \mathrm{DSC}$ scan of $P d_{77.5} \mathrm{Cu}_{6} S i_{16.5}$ measured with a heat rate of $\mathrm{T}=20 \mathrm{~K} / \mathrm{min}$. The glass transition and crystallization temperatures can be estimated from the heat hump and crystallization peaks respectively: $\mathrm{Tg}=621 \pm 10 \mathrm{~K}$ and $\mathrm{TX}=685 \pm 10 \mathrm{~K}$

Figure 5.3 XRD spectra of $P d_{77.5} C u_{6} S i_{16.5}$ samples. i) As cast, ii) creep at $\mathrm{T} / \mathrm{Tg}=0.8, \sigma=12$, iii) creep at TTg $=0.89, \sigma=12$, iv) creep at T/Tg $=0.94, \sigma=36 \mathrm{MPa}, \mathrm{v}$ ) creep at T/Tg $=0.97, \sigma=$ $12 \mathrm{MPa}$. Inset: $\mathrm{V}^{*}$ ) creep at $\mathrm{T} / \mathrm{Tg}=0.97, \sigma=12 \mathrm{MPa}$ before polishing.

Figure 5.4 (a) Creep curves at $\mathrm{T} / \mathrm{Tg}=0.85$ and $\sigma=2 \mathrm{MPa}$ (black line), $\sigma=8 \mathrm{MPa}$ (red line), and $\sigma=12 \mathrm{MPa}$ (green line.) (b) Creep curves at $\mathrm{T} / \mathrm{Tg}=0.89$ and $\sigma=2 \mathrm{MPa}$ (black line), $\sigma=8 \mathrm{MPa}$ (red line), and $\sigma=12 \mathrm{MPa}$ (green line.) (c) Creep curves at $\mathrm{T} / \mathrm{Tg}=0.92$ and $\sigma=2 \mathrm{MPa}$ (black line), $\sigma=8 \mathrm{MPa}$ (red line), and $\sigma=12 \mathrm{MPa}$ (green line.) (d) Creep curves at $\mathrm{T} / \mathrm{Tg}=0.94$ and $\sigma=$ $2 \mathrm{MPa}$ (black line), $\sigma=8 \mathrm{MPa}$ (red line), and $\sigma=12 \mathrm{MPa}$ (green line.).

Figure 5.5. Stress-Temperature map. Each square represents the waiting time distribution before and after the crossover time tcross under the stress and temperature that corresponds with it position. The background color describes the magnitude of tcross.

Figure 5.6. Exponents of the waiting time distributions in the stress-temperature map. Red symbols represents the exponent of the distribution before the crossover and green symbols correspond to the distribution after the crossover. The dashed lines represent the intervals $\tau 1=-1.5 \pm 0.1$ and $\tau 2=-0.8 \pm 0.1$.

Figure 5.7 Creep measurements used to investigate the quantitative dependence of tcross with the stress and temperature. (a) Tests done keeping a constant stress and sweeping the temperature in the range $T / T g=0.8-0.97$. (b) Set of measurements performed keeping aconstant temperature $T / T g=0.94$ and sweeping the stress in the range $\sigma=2-36 \mathrm{MPa}$.

Figure 5.8 (a) Dependence of tcross (red) and $t \varepsilon$ (blue) with the temperature at a fixed stress. (b) Dependence of tcross (red) and $t \varepsilon$ (blue) with the stress at a fixed temperature.

Figure 5.9 EDX pattern of the 2605SA1 alloy. The dispersive X-ray shows emission lines corresponding to $\mathrm{Fe}, \mathrm{Si}$ and $\mathrm{C}$

Figure 5.10. DSC scan of 2605A1 measured with a heat rate of $T=20 \mathrm{~K} / \mathrm{min}$. The Curie and crystallization temperatures can be estimated from the heat hump and crystallization peaks respectively: $T c=674 \pm 10 \mathrm{~K}$ and $T X=782 \pm 10 \mathrm{~K}$. However the glass transition temperature is difficult to estimate accurately. .81

Figure 5.11 (a) Temperature scans of 2605A1 ribbons performed for several constant mechanical loads at a constant heating rate $T=10 \mathrm{~K} / \mathrm{min}$. (b) Temperature in which the strain rate leaves the 
linear regime $T \varepsilon$ as a function of the mechanical load calculated from the curves shown in (a). A linear regression to the data yields a relationship: $\operatorname{Tg} \sigma=(694 \pm 6)-(2.5 \pm 0.3) \sigma$.

Figure 5.12 (a) Hysteresis loops of 2605SA1 measured by means of VSM. The different curves represent the magnetisation curves along the three main axes of symmetry of the ribbon: length (red), width (blue) and thickness (green). (b) Magnetisation vs Temperature curve M(T) vs T of 2605SA1 measure under a small field $\mathrm{H}=300$ Oe. The inset shows the histeresis curves measured along the width direction at the temperatures: $373,473,573$ and $673 \mathrm{~K}$. .83

Figure 5.13. XRD spectra of the 2605SA1 alloy. i) As cast ii) $\mathrm{T} / \mathrm{Tg}=0.8, \sigma=15 \mathrm{MPa}$, iii) $\mathrm{T} / \mathrm{Tg}=$ $0.95, \sigma=15 \mathrm{MPa}$, iv) $T / \mathrm{Tg}=0.95, \sigma=15 \mathrm{MPa}, \mathrm{v}) \mathrm{T} / \mathrm{T} g=0.8, \sigma=15 \mathrm{MPa}, H=500, \theta=$ $0, \phi=0$, vi) $T T g=0.8, \sigma=15 \mathrm{MPa}, H=200, \theta=0, \phi=45$. Inset: Fully crystallized sample annealed 10 minutes above TX. 84

Figure 5.14 (a) Creep tests of 2605SA1 ribbons, all subjected to the same mechanical stress ( $\sigma=$ $15 \mathrm{MPa}$ ) and different temperatures. (b) Waiting time distribution calculated from the creep curves shown in (a). The dashed line represents a perfect power law with exponent $\tau=-0.8$. 86

Figure 5.15. Waiting time distributions before and after the crossover time, for the creep measurements on Fe -rich 2605SA1 under several temperatures: a) $T / T g=0.8, \sigma=15 \mathrm{MPa}$. (b) $T / T g=0.85, \sigma=15 \mathrm{MPa}$. (c) $T / T g=0.9, \sigma=15 \mathrm{MPa}$ (d) $T / T g=0.95, \sigma=15 \mathrm{MPa} \quad$ (e) $T / T g=0.97, \sigma=15 \mathrm{MPa}$ (f) Fit of the power law exponents before and after the crossover calculated from the curves (a)-(e), the dashed lines represent the intervals $\tau 1=-1.5 \pm$ 0.1 and $\tau 2=-0.8 \pm 0.1$. 87

Figure 5.16 Dependence of $t$ cross and $t \varepsilon$ with the temperature at a fixed stress $\sigma=15 \mathrm{MPa} . .88$

Figure 5.17 (a) Effective energy barrier as a function of experimental time calculated from the Transition State Theory for the creep measurements done at $\sigma=15 \mathrm{MPa}$ and several temperatures. (b) Residuals Vs time. The residuals are calculated as the difference between the energy values and a LOESS fit. (c) Probability distribution of the residuals for the different temperatures. (d) Evolution of $W$ as a function of the temperature.

Figure 5.18 (a) Creep tests of 2605SA1 ribbons subjected to different mechanical stresses and kept at the same temperature $\mathrm{T} / \mathrm{Tg}=0.8$. (b) Waiting time distribution calculated from the creep curves shown in (a). The dashed line represents a perfect power law with exponent $\tau=-0.8 \ldots 91$

Figure 5.19 Waiting time distributions before and after the crossover time, for the creep measurements of 2605SA1 ribbons measured at temperature $T / T g=0.8$ and under several mechanical stresses . (a) $\sigma=15 \mathrm{MPa}$. (b) $\sigma=15 \mathrm{MPa}$. (c) $\sigma=15 \mathrm{MPa}$ (d) $\sigma=15 \mathrm{MPa}$ (e) $\sigma=$ $15 \mathrm{MPa}$ (f) Fit of the power law exponents before and after the crossover calculated from the curves (a)-(e), the dashed lines represent the intervals $\quad \tau 1=-1.5 \pm 0.1$ and $\tau 2=-0.8 \pm$ 0.1 . 
Figure 5.20 (a) Dependence of $t$ cross and $t \varepsilon$ with stress for a fixed temperature $T / T g=0.8$ for the 2605SA1 alloy (b) $W$ as a function of the mechanical stress for the same composition at the same temperature.

Figure 5.21 (a) Creep measurements of 2605SA1 perfermed at $T / T g=0.8, \sigma=15$ for different intensities of magnetic field oriented along the width direction $\theta=0, \phi=0$. (b) Double logarithmic representation of the waiting time distribution calculated from the creep measurements shown in (a) .95

Figure 5.22 Waiting time distributions before and after the crossover time, for the creep measurements at temperature $T / \mathrm{Tg}=0.8, \sigma=15 \mathrm{MPa}$ and under several intensities of magnetic field along the width direction $\theta=0, \phi=0$. (a) $H=0$ Oe. (b) $H=150$ Oe. (c) $H=340$ Oe (d) $H=500$ Oe (e) $H=640$ Oe (f) Fit of the power law exponents before and after the crossover calculated from the curves (a)-(e), the shaded areas represent the intervals $\tau 1=-1.5 \pm$ 0.1 and $\tau 2=-0.8 \pm 0.1$. .96

Figure 5.23 (a) Dependence of tcross and $t \varepsilon$ with the magnetic field intensity applied along the width direction $\theta=0, \phi=0$, calculated from creep measurements on 2605SA1 ribbons for a fixed temperature $T / T g=0.8$ and several stresses (b) $W$ as a function of magnetic field intensity, applied along the width direction $\theta=0, \phi=0$, calculated from creep measurements on 2605SA1 ribbons for a fixed temperature $T / T g=0.8$ and stress $\sigma=15 \mathrm{MPa}$.

Figure 5.24 (a) Creep measurements of 2605SA1 perfermed at $T / T g=0.8, \sigma=25$ for different intensities of magnetic field oriented along the width direction $\theta=0, \phi=0$. (b) Double logarithmic representation of the waiting time distribution calculated from the creep measurements shown in (a)

Figure 5.25 Waiting time distributions before and after the crossover time, for the creep measurements at temperature $T / \mathrm{Tg}=0.8, \sigma=25 \mathrm{MPa}$ and under several intensities of magnetic field along the width orientation $\theta=0, \phi=0$. (a) $H=0$ Oe. (b) $H=150$ Oe. (c) $H=340$ Oe (d) $H=500$ Oe (e) $H=640$ Oe (f) Fit of the power law exponents before and after the crossover calculated from the curves (a)-(e), the dashed represent the intervals $\tau 1=-1.5 \pm 0.1$ and $\tau 2=$ $-0.8 \pm 0.1$.

Figure 5.26 (a) Dependence of tcross and $t \varepsilon$ with the magnetic field intensity, applied along the width direction $\theta=0, \phi=0$, for a fixed temperature $T / T g=0.8$ and stress $\sigma=25 \mathrm{MPa}$ (b) FWHM of the energy residual distribution as a function of magnetic field intensity, applied along the width direction $\theta=0, \phi=0$, for a fixed temperature $T / T g=0.8$ and stress $\sigma=25$ MPa.

Figure 5.27 Schematic view of the magnetic field orientation with respect to the mechanical stress and ribbon axes used in the experiments described in the current subsection..... 101 
Figure 5.28 (a) Creep measurements of 2605SA1 perfermed at $T / T g=0.8, \sigma=15 \mathrm{MPa}, H=$ $500 O e, \phi=0$ ) for different angles $\theta$. (b) Double logarithmic representation of the waiting time distribution calculated from the creep measurements shown in (a) 102

Figure 5.29 Waiting time distributions before and after the crossover time, for the creep measurements of 2605SA1 performed at $T / T g=0.8, \sigma=15 \mathrm{MPa}, H=500 \mathrm{Oe}, \phi=0$ ) for different angles $\theta$. (a) $\theta=0$ Oe. (b) $\theta=20^{\circ}$. (c) $\theta=45^{\circ}$ (d) $\theta=60^{\circ}$ (e) $\theta=90^{\circ}$ (f) Best fit of the power law exponents before and after the crossover calculated from the curves (a)-(e), the coloured areas represent the intervals $\tau 1=-1.5 \pm 0.1$ and $\tau 2=-0.8 \pm 0.1$. 103

Figure 5.30 (a) Dependence of tcross and $t \varepsilon$ with the angle $\theta$ for creep experiments performed on 2605SA1 at , $T / T g=0.8, \sigma=15 \mathrm{MPa}, H=500 \mathrm{Oe}, \phi=0$ ) (b) $W$ as a function of the angle $\theta$ for creep experiments performed on 2605SA1 at , $T / T g=0.8, \sigma=15 \mathrm{MPa}, H=500 \mathrm{Oe}, \phi=0104$

Figure 5.31 Schematic view of the magnetic field orientation with respect to the mechanical stress and ribbon axes used in the experiments shown in the current subsection 105

Figure 5.32 (a) Creep measurements of 2605SA1 perfermed at $T / T g=0.8, \sigma=15 \mathrm{MPa}, H=$ $1200 e, \theta=0$ ) for different angles $\phi$. (b) Double logarithmic representation of the waiting time distribution calculated from the creep measurements shown in (a). 106

Figure 5.33 Waiting time distributions before and after the crossover time, for a sample of the creep measurements of 2605SA1 performed at $T / \mathrm{Tg}=0.8, \sigma=15 \mathrm{MPa}, \mathrm{H}=120 \mathrm{Oe}, \theta=0$ ) for different angles $\phi$. (a) $\phi=-90$ Oe. (b) $\phi=-60^{\circ}$. (c) $\phi=-30^{\circ}$ (d) $\phi=30^{\circ}$ (e) $\phi=90^{\circ}$ (f) Power law exponent fit before and after the crossover calculated from the curves (a)-(e), the coloured areas represent the intervals $\tau 1=-1.5 \pm 0.1$ and $\tau 2=-0.8 \pm 0.1$. 107

Figure 5.34 (a) Dependence of tcross and $t \varepsilon$ with the angle $\phi$ for creep experiments performed on 2605SA1 at , $T / T g=0.8, \sigma=15 \mathrm{MPa}, H=1200 e, \theta=0$ ) (b) FWHM of the energy residual distribution as a function of the angle $\theta$ for creep experiments performed on 2605SA1 at , $T / T g=$ $0.8, \sigma=15 \mathrm{MPa}, H=120 \mathrm{Oe}, \theta=0$ ) 108

Figure 5.35 EDX spectra of $\mathrm{Fe}_{2} \mathrm{Co}_{73} \mathrm{Si}_{10} \mathrm{~B}_{15}$ 109

Figure 5.36 (a) Hysteresis loops measured along the three main axes of symmetry of the Co-rich alloy by means of Vibrating Sample Magnetometer. (b) Differential Scanning Calorimeter measurement of the Co-rich alloy..... 110

Figure 5.37 (a) XRD spectra of the Co-rich alloy. i) As cast, ii) stress-strained $H=340$ Oe, iii) stressstrained at $H=500 \mathrm{Oe}$, iv) stress-strained at $H=700 \mathrm{Oe}$, v) stress-strained $H=950 \mathrm{Oe}$. (b) XRD 
spectra of the Fe-rich alloy. i) As cast, ii) stress-strained at $H=340 \mathrm{Oe}$, iii) stress-strained at $H=$ $500 \mathrm{Oe}$, iv) stress-strained at $H=700 \mathrm{Oe}$, v) stress-strained at $H=950 \mathrm{Oe}$

Figure 5.38 (a) stress-strain curves of $\mathrm{Fe}_{2} \mathrm{Co}_{73} \mathrm{Si}_{10} \mathrm{~B}_{15}$ under different intensities of magnetic field applied along the out-of-plane direction $\left(\theta=90^{\circ}\right)$. (b) stress strain curves of 2605SA1 under different intensities of magnetic field applied along the out-of-plane direction $\left(\theta=90^{\circ}\right)$. (c) stressstrain curves of $\mathrm{Fe}_{2} \mathrm{Co}_{73} \mathrm{Si}_{10} \mathrm{~B}_{15}$ under different intensities of magnetic field applied along the width direction $(\theta=0)$. (d) stress-strain curves of 2605SA1 under different intensities of magnetic field applied along the width direction $(\theta=0)$. All test are perfomed with a force rate of 30 $\mathrm{mN} / \mathrm{min}$ and at a constant temperature $\mathrm{T}=300 \mathrm{~K}$. 112

Figure 5.39 (a) Macroscopic Young modulus Emacro as a function of the intensity of magnetic field for the CoFeSiB metallic glass. (b) Macroscopic Young modulus Emacro as a function of the intensity of magnetic field for the2605SA1 metallic glass. 113

Figure 5.40 Distribution of normalized modulus $P(E)$ of $\mathrm{Fe}_{2} \mathrm{Co}_{73} \mathrm{Si}_{10} B_{15}$ (a) and 2605SA1 (b) alloys under several values of magnetic field applied along the $\theta=0$ (squares) and $\theta=90^{\circ}$ (triangles) directions. Figure 5.40(b) Inset. $P(E)$ for $\theta=0, H=200,340,500,640$ Oe.

Figure 5.41 (a) Distribution of effective modulus of the 2605SA1 alloy under magnetic loading along $\theta=0$ for different intensities of applied field. (b) Distribution of effective modulus of 2605SA1, under an applied field of $\mathrm{H}=500$ Oe separated in two regimes according to the kink that takes places at $\sigma k=12.5 \mathrm{MPa}:$ P(Eeff) for $\sigma<\sigma k$ (red) and $P$ (Eeff) for $\sigma<\sigma k$ (blue).....

Figure 6.1 Natural logarithm of tcross as a function of the $\mathrm{Tg} / \mathrm{T}$ plotted in an Angell plot fashion. Dashed lines represent Least Squares fits calculated in the two regimes $\mathrm{T} / \mathrm{Tg}<0.9$ and $\mathrm{T} / \mathrm{Tg}>$ 0.9 .

Figure 6.2 (a) Waiting time distribution yield upon creep measurements performed at $\sigma=8 \mathrm{MPa}$ and $\mathrm{TTg}=0.85,0.89,0.92$ and 0.94. (b) Waiting time distribution obtained from creep measurements performed at $\mathrm{TTg}=0.89$ and $\sigma=2,8$ and $12 \mathrm{MPa}$

Figure 6.3. (a) Evolution of tcross and $t \varepsilon$ as a function of magnetic field intensity calculated from creep measurements of 2605SA1 measured at $\sigma=15 \mathrm{MPa}, \mathrm{T} / \mathrm{Tg}=0.8$. (b) Evolution of $W$ as a function of magnetic field intensity calculated from creep measurements of 2605SA1 measured at $\sigma=15 \mathrm{MPa}, \mathrm{T} / \mathrm{Tg}=0.8$. (c) Evolution of tcross and $t \varepsilon$ as a function of magnetic field intensity calculated from creep measurements of 2605SA1 measured at $\sigma=25 \mathrm{MPa}, \mathrm{T} / \mathrm{Tg}=0.8$ (d) (b) Evolution of $W$ as a function of magnetic field intensity calculated from creep measurements of 2605SA1 measured at $\sigma=25 \mathrm{MPa}, \mathrm{T} / \mathrm{Tg}=0.8$.

Figure 6.4 (a) Dependence of with the angle $\phi$ for creep experiments performed on 2605SA1 at $\mathrm{TTg}=0.8, \sigma=15 \mathrm{MPa} H=120 \mathrm{Oe}$, (b) $W$ as a function of the angle $\phi$ for creep experiments performed on 2605SA1 TTg $=0.8, \sigma=15 \mathrm{MPa} H=120$ 0e. 
Figure 6.5 .Schematic illustration of the relative orientation of the STZ stress field and the magnetization vector in a domain wall oriented $45^{\circ}$ respect the vertical axis. Dashed lines represent domain walls and red and green arrows indicate the STZ stress field and magnetization vector respectively.

Figure A.1 (a) evolution of $t_{\dot{\varepsilon}}$ as function of stress calculated from creep measurements of 2605SA1 at $T / \mathrm{Tg}=0.8$, calculated using $\left(\alpha_{1}=10, \alpha_{2}=5\right)$, (b) evolution of $t_{\dot{\varepsilon}}$ as function of stress calculated from creep measurements of 2605SA1 at $T / T g=0.8$, calculated using $\left(\alpha_{1}=20, \alpha_{2}=\right.$ 10 ), (c) evolution of $t_{\dot{\varepsilon}}$ as function of stress calculated from creep measurements of 2605SA1 at $\frac{T}{T g} 0.8$, calculated using $\left(\alpha_{1} 40, \alpha_{2} 20\right)$ 136

Figure A.2 (a) evolution of normalized $t_{\dot{\varepsilon}}$ as function of stress calculated from creep measurements of 2605SA1 at $T / T g=0.8$, for different thresholds $\left(\alpha_{1}=10, \alpha_{2}=5\right),\left(\alpha_{1}=20, \alpha_{2}=\right.$ $10)$ and $\left(\alpha_{1}=40, \alpha_{2}=20\right)$. (b) evolution of normalized $t_{\dot{\varepsilon}}$ as function of temperature calculated from creep measurements of 2605SA1 at stress $\sigma=15 \mathrm{MPa}$ for different thresholds $\left(\alpha_{1}=10, \alpha_{2}=5\right),\left(\alpha_{1}=20, \alpha_{2}=10\right)$ and $\left(\alpha_{1}=40, \alpha_{2} 20\right)$. .137

Figure B.1 Evolution of Was function of mechanical stress $\sigma$ at constant T/T $(\sigma)$ .145 
